SAND92-2186 • UC-814

Unlimited Release

Printed March 1995

Yucca Mountain Site Characterization Project

\title{
Scenarios Constructed for Nominal Flow in the Presence of a Repository at Yucca Mountain and Vicinity
}

\author{
G. E. Barr, Regina L. Hunter, Ellen Dunn, A. Flint
}

Prepared by

Sandia National Laboratories

Albuquerque, New Mexico 87185 and Livermore, California 94550

for the United States Department of Energy

under Contract DE-AC04-94AL85000

Approved for public release distribution is unlimited.
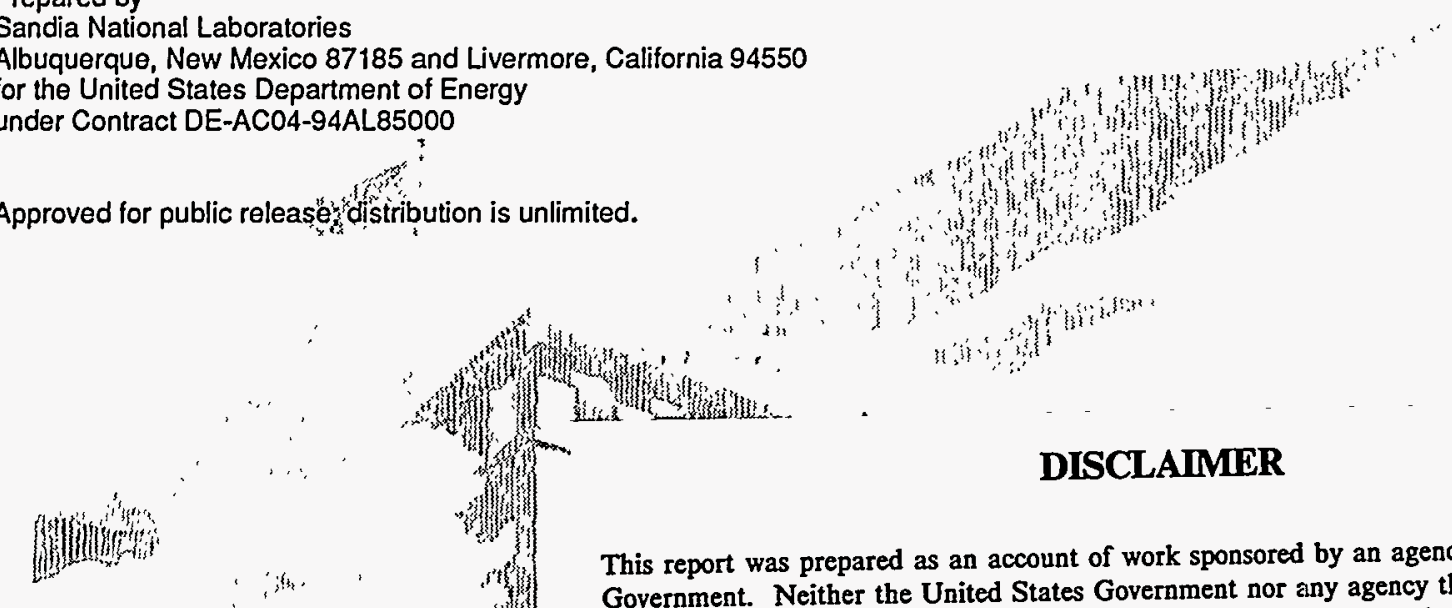

\section{DISCLAIMER}

This report was prepared as an account of work sponsored by an agency of the United States Government. Neither the United States Government nor any agency thereof, nor any of their Government. Neres any warranty, express or implied, or assumes any legal liability or responsibility for the accuracy, completeness, or usefulness of any information, apparatus, product, or bility for the accuracy, completeness, or usefulness not infringe privately owned rights. Reference herein to any specific commercial product, process, or service by trade name, trademark, ence herein to any specific commercial product, process, or service by trade name, trademark,

: manufacturer, or otherwise does not necessarily constitute or any agency thereof. The views mendation, or favoring by the Unithed herein do not necessarily state or reflect those of the and opinions of authors expressed herey dotes Government or any agency thereof.

(1) a)

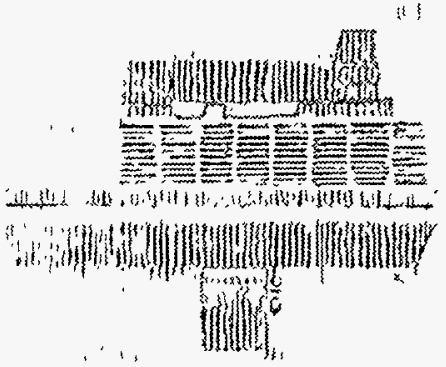

SF 2900O(8.81)

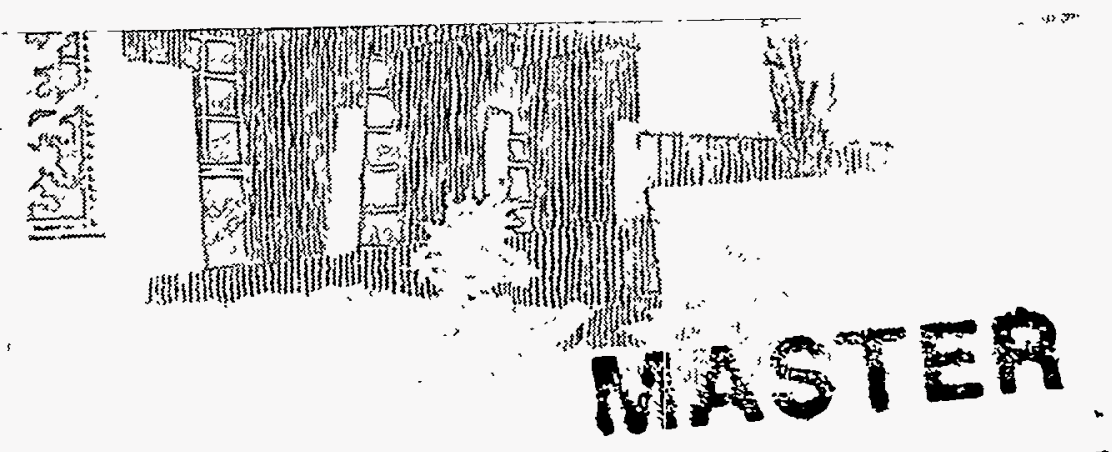

in. III

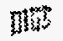
* 
"Prepared by Yucca Mountain Site Characterization Project (YMSCP) participants as part of the Civilian Radioactive Waste Management Program (CRWM). The YMSCP is managed by the Yucca Mountain Project Office of the U.S. Department of Energy, DOE Field Office, Nevada (DOE/NV). YMSCP work is sponsored by the Office of Geologic Repositories (OGR) of the DOE Office of Civilian Radioactive Waste Management (OCRWM)."

Issued by Sandia National Laboratories, operated for the United States Department of Energy by Sandia Corporation.

NOTICE: This report was prepared as an account of work sponsored by an agency of the United States Government. Neither the United States Government nor any agency thereof, nor any of their employees, nor any of their contractors, subcontractors, or their employees, makes any warranty, express or implied, or assumes any legal liability or responsibility for the accuracy, completeness, or usefulness of any information, apparatus, product, or process disclosed, or represents that its use would not infringe privately owned rights. Reference herein to any specific commercial product, process, or service by trade name, trademark, manufacturer, or otherwise, does not necessarily constitute or imply its endorsement, recommendation, or favoring by the United States Government, any agency thereof or any of their contractors or subcontractors. The views and opinions expressed herein do not necessarily state or reflect those of the United States Government, any agency thereof or any of their contractors.

Printed in the United States of America. This report has been reproduced directly from the best available copy.

Available to DOE and DOE contractors from Office of Scientific and Technical Information

PO Box 62

Oak Ridge, TN 37831

Prices available from (615) 576-8401, FTS 626-8401

Available to the public from

National Technical Information Service

US Department of Commerce

5285 Port Royal Rd

Springfield, VA 22161

NTIS price codes

Printed copy: A10

Microfiche copy: A01 


\section{DISCLAIMER}

Portions of this document may be illegible in electronic image products. Images are produced from the best available original document. 
SAND92-2186

Unlimited Release

March 1995

Category UC-814

\title{
Scenarios Constructed for Nominal Flow \\ In the Presence of a Repository At Yucca Mountain and Vicinity
}

\author{
G. E. Barr \\ Regina L. Hunter \\ Ellen Dunn \\ Sandia National Laboratories
}

A. Flint

Abstract

Scenario development for the system performance assessment of the Yucca Mountain Site Characterization Project defines a scenario as a well-posed problem connecting an initiating event with radionuclide release to the accessible environment by a logical and physically possible combination or sequence of features, events, and processes. Drawing on the advice and assistance of the Project's principal investigators (PIs), a collection of release scenarios initiated by the nominal ground-water flow occurring in the vicinity of the potential Yucca Mountain highlevel-waste repository is developed and described in pictorial form. This collection of scenarios is intended to provide a framework to assist PIs in recognizing essential field and calculational analyses, to assist performance assessment in providing guidance to site characterization, and to continue the effort to exhaustively identify all features, events, and processes important to releases. It represents a step in the iterative process of identifying what details of the potential site are important for safe disposal.

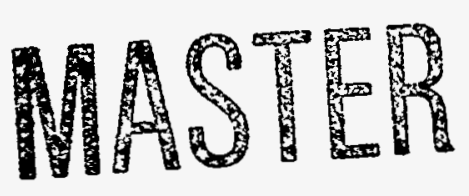




\section{ACKNOWLEDGMENTS}

Jeanette Anderson of Creative Computer Services and Kassandra Sanchez, Vickie Greenier, and Dominic Martinez of Sandia National Laboratories prepared the figures and tree segments in this report. The authors appreciate this support.

This report was prepared under the Yucca Mountain Site Characterization Project WBS number 1.2.5.4.1, QA Grading Report \# 1.2.5.4.1. The planning document that guided this work activity was WA-0036. The information and data documented in this report are not qualified and will not be used in licensing the repository.

This work was supported by the United States Department of Energy under Contract DE-AC04-94AL85000. 
SUMMARY

1.1 Conditions Imposed by the Repository

1.1.1 Movement of Vaporization Isotherm

1.1.2 Mine Ventilation

1.1.3 Condensation Cap

1.2 Reading This Report

2 RUNOFF-PRODUCING EVENTS

2.1 Runoff to Washes

2.1.1.1 Surface-Channel Flow

Saturated Flow to Bedrock

Fracture Flow Through Bedrock, Hot Repository .................................................... 31

Path 1.1

Path 1.2

Path 1.3

Path 1.4

Fracture Flow Through Bedrock, Cold Repository .

Path 1.5

Path 1.6

Unsaturated Flow in Bedrock, Hot Repository

Path 2.1

Path 2.2

Path 2.3

Path 2.4

Unsaturated Flow in Bedrock, Cold Repository; Path 2.5

Hot Repository, Paths $\widehat{3.1}$ through $\widehat{3.4}$................................................... 74

Cold Repository, Paths 3.5 through 3.7 ..................................................... 74

Unsaturated Flow to Bedrock, Paths 4.1 through 6.6 ................................................ 77

2.1.1.2 Subsurface Flow, Tree Segment 7.......................................................................... 86

2.1.1.3 Interception by Terraces, Tree Segments 8 through 10................................................ 91

2.1.2 Washes Without Faults...................................................................................................... 99

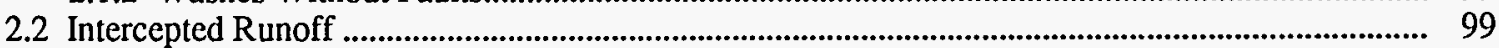

2.2.1 Fracture Flow to Topopah Spring Units (Perched Flow)..................................................... 113

2.2.1.1 Hot Repository, Paths 13.1 Through 13.4 ....................................................... 113

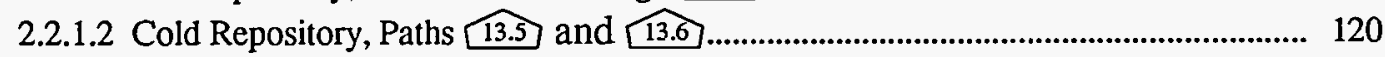

2.2.2 Fracture Flow to Topopah Spring Units (Matrix Imbibition)................................................ 120

2.2.2.1 Hot Repository, Paths $\widehat{14.1}$ Through $\widehat{14.4 \ldots \ldots . . . . . . . . . . . . . . . . . . . . . . . . . . . . . . . . . . . . . . . . . . . . . . . . . . . . . . . . . ~} 122$

2.2.2.2 Cold Repository, Path $\widehat{14.5}$.............................................................................. 127

2.2.3 Flow Into Shallow Fractures and Direct Infiltration ............................................................. 127 


\section{TABLE OF CONTENTS (continued)}

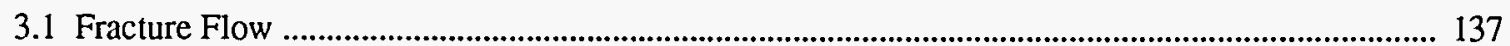

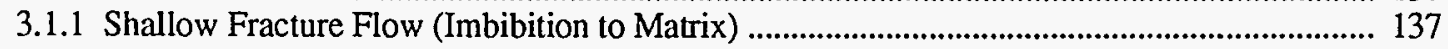

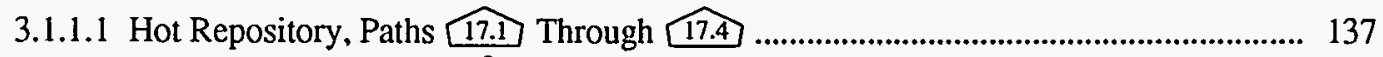

3.1.1.2 Cold Repository, Path $\widehat{17.5}$...................................................................................... 146

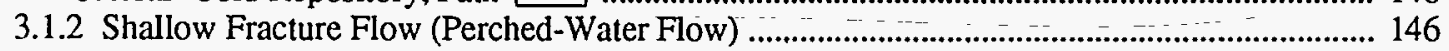

3.1.2.1 Hot Repository, Paths $\widehat{18.1}$ Through $\widehat{18.4}$............................................................ 146

3.1.2.2 Cold Repository, Path $\widehat{18.5}$...................................................................................... 150

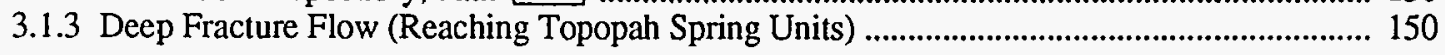

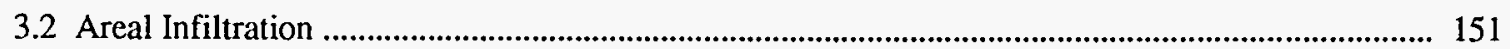

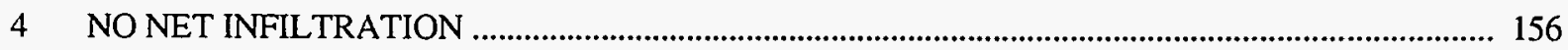

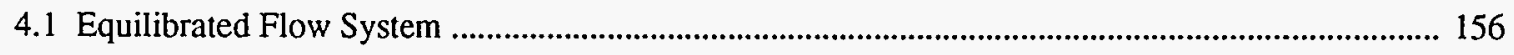

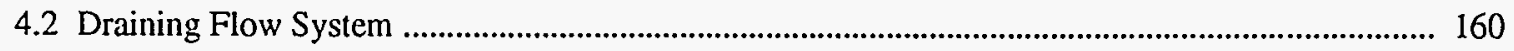

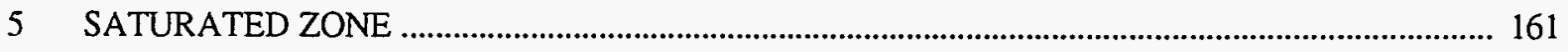

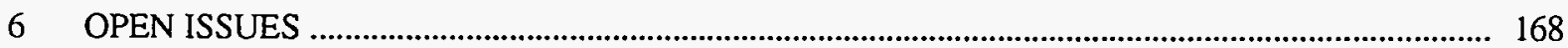

6.1 Thermal-Mechanical-Hydrologic Coupling ...................................................................... 168

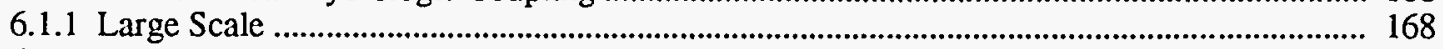

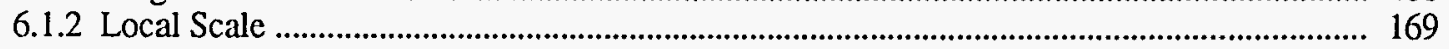

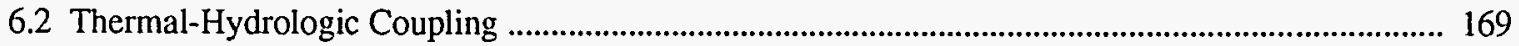

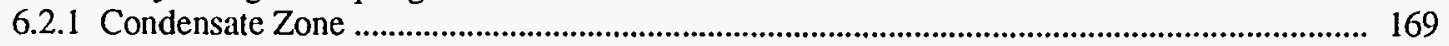

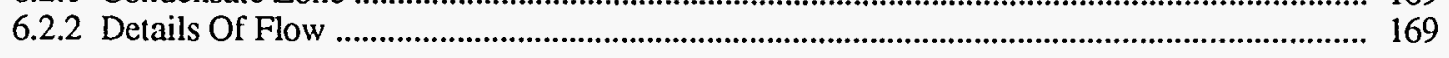

6.3 Thermal-Mechanical-Chemical-Hydrologic Coupling ................................................................... 169

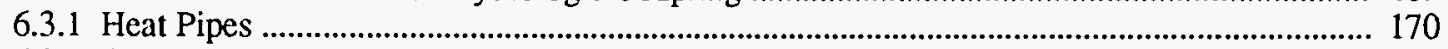

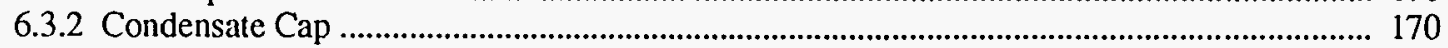

6.3.3 Contaminant Mobilization ................................................................................................. 170

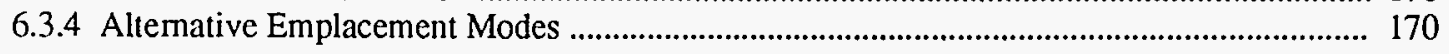

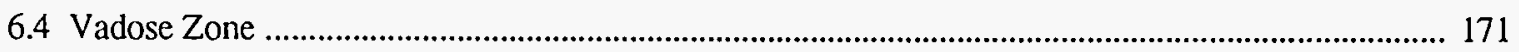

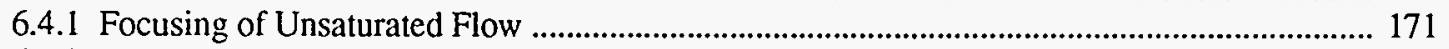

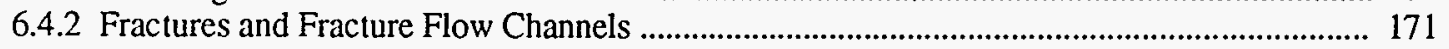

6.4.3 Evolution of Fracture Flow Channels ............................................................................. 171

6.4.4 Geostatistical Realizations of Hydrologic Properties ................................................................... 171

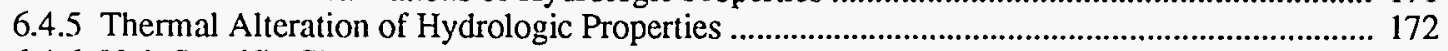

6.4.6 Unit Specific Changes on the Large Scale .................................................................. 172

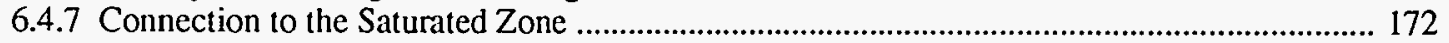

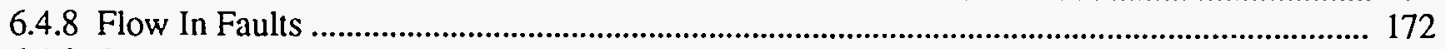

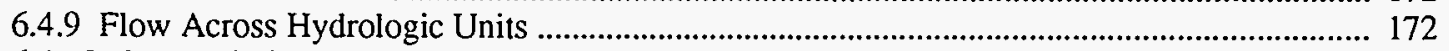

6.4.10 Flow Within Fracture Networks ,................................................................................ 172 


\section{TABLE OF CONTENTS (concluded)}

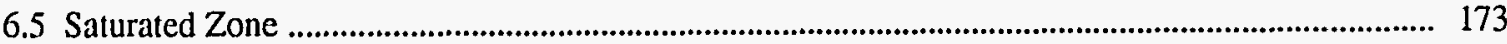

6.5.1 Fault Controls on the Flow System ........................................................................................ 173

6.5.2 Coupling Between the Tuff And Carbonate Aquifers ................................................................ 173

6.5.3 Thermal-Chemical Alteration .................................................................................................... 173

6.5.4 Dispersion In Fractured, Porous Media ................................................................................. 174

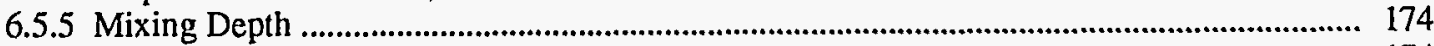

6.5.6 Hydrologic Properties of Unsaturated Units ........................................................................ 174

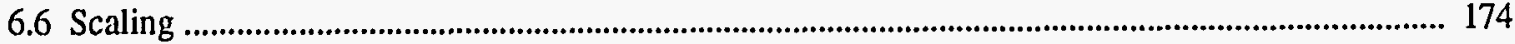

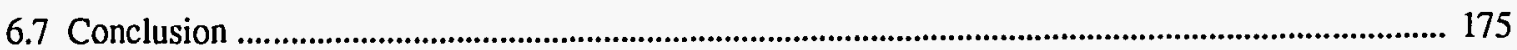

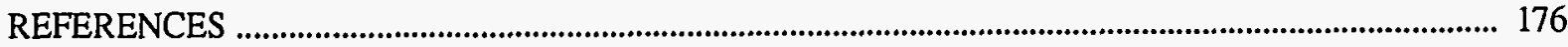




\section{LIST OF FIGURES}

Figure

1 Decision tree illustrating where analyses of features, events, and processes are necessary to resolve how the repository system and mountain perform

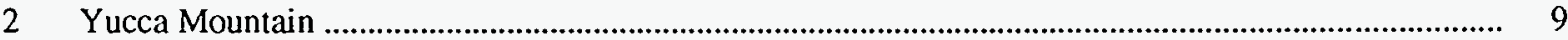

$3 \quad$ Upper layers of the event tree for nominal flow ............................................................................... 16

$4 \quad$ Nominal Flow, Runoff-Producing Events, Runoff to Washes.................................................................. 23

5 Intermediate layers of the event tree for nominal flow, showing major branches for washes without

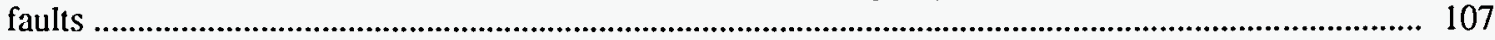




\section{LIST OF SKETCHES}

Sketch

Page

G Near vicinity of repository, showing waste containers, drifts and backfills, and stress-induced radial and concentric fractures...

G1 Thermally induced rapid downward flow of water through the repository to form a semi-perched zone below

I Diagrammatic cross section of waste container, drift and backfill, and induced and previously existing fractures

C Runoff-producing events

$\mathrm{Cl}$ Strata dependence of the mode of infiltration, i.e., saturated flow in fractures vs. unsaturated flow in matrix, for the stratigraphic units at the north end of the repository

C2 Strata dependence of the mode of infiltration, i.e., saturated flow in fractures vs. unsaturated flow in matrix, for the stratigraphic units at the south end of the repository

D Surface-flow channel in a wash with faults

E Small-scale diagrammatic cross section of a wash with faults, showing unsaturated flow through the alluvium

E1 Small-scale diagrammatic cross section of a wash with faults, showing saturated flow through the alluvium

F1 Large-scale diagrammatic cross section of a wash with faults, showing saturated flow through the alluvium followed by downward and lateral flow in faults and fractures to repository depth

F2 Saturated flow in alluvium followed by unsaturated flow in bedrock 28

F3 Saturated flow in faults to repository depth from the alluvium in a wash with faults

F Large-scale diagrammatic cross section of a wash with faults, showing unsaturated flow through the alluvium followed by downward and lateral flow in faults and fractures to repository depth ..

$\mathrm{H}$ Idealized view of the interaction between fracture flow from the wash and the condensation cap formed by heat-driven two-phase fluid flow around a repository

I1 Diagrammatic cross section of waste and drift showing the vaporization isotherm and dryout zone ....... 36

$\mathrm{J}$ Saturated return of liquids from the condensation cap to the repository

$\mathrm{K}$ Degradation of the EBS and mobilization of contaminants in the presence of voluminous reflux that partially submerges the waste container

K1 Degradation of the EBS and mobilization of contaminants in the presence of meager reflux that drips onto the waste container

M Transport of contaminants from the repository to the water table, showing saturated fracture flow to the waste container and transport in saturated fractures to the water table

J1 Unsaturated return of liquids from the condensation cap to the repository 


\section{LIST OF SKETCHES (continued)}

Sketch

K2 Degradation of the EBS and mobilization of contaminants in the presence of unsaturated flow

M1 Transport of contaminants from the repository to the water table, showing unsaturated flow to the waste container and unsaturated transport to the water table

H1 Fracture flow from a wash with faults

J2 Large-scale view of the return flow of liquid and vapor to the repository and the interaction with fracture flow from a wash with faults

J5 Episodic unsaturated flow from runoff reaching the waste containers

H2 Details of heat-pipe behavior near the backfilled drift

I2 Vaporization and dryout near the waste container as a result of heat-pipe behavior

M2 Transport of contaminants from the repository to the water table, showing unsaturated flow to the waste containers followed by unsaturated transport to the water table

$\mathrm{N}$ Imbibition of liquids into the matrix from fractures to form an unsaturated-flow plume

G5 Unsaturated flow plume entering a cold repository

K6 Degradation of the EBS and mobilization of contaminants in the presence of blind, saturated fractures leaking to the unsaturated flow system

H3 Saturated flow to bedrock followed by fracture flow to the repository

I3 Fracture flow to the repository

K3 Degradation of the EBS and mobilization of contaminants, showing both saturated fracture flow and unsaturated flow from blind fractures to the waste container

G2 Interaction between the condensation cap and an unsaturated flow plume descending from a wash .

J3 Saturated return flow from the condensation cap to the waste container in the presence of an unsaturated-flow plume from a wash with faults

M3 Transport of contaminants from the repository to the water table, showing reflux from the condensation cap to the repository followed by both saturated and unsaturated transport to the water table

J4 Unsaturated return flow from the condensation cap to the waste container

K5 Degradation of the EBS and mobilization of contaminants in an unsaturated flow field that leads to an unsaturated contaminant plume

G3 Unsaturated flow plume interacting with the dryout zone

G4 Interaction of heat pipe around waste containers, with two-phase convective flow and capillary return flow in the matrix, with the unsaturated-flow plume 


\section{LIST OF SKETCHES (continued)}

Sketch

Page

K4 Degradation of the EBS and mobilization of contaminants in the presence of a saturated flux (composite model) that reaches the waste container and produces a saturated contaminant flow plume . 75

H4 Migration of fluids laterally to interact with the repository 76

F4 Unsaturated flow in alluvium followed by unsaturated flow in bedrock .............................................. 84

F5 Unsaturated flow in alluvium followed by saturated flow in faults 85

E2 Small-scale diagrammatic cross section of a wash with faults, showing a zone of unsaturated flow that is locally unconnected with the flow channel.

E3 Small-scale diagrammatic cross section of a wash with faults, showing a zone of saturated flow that is locally unconnected with the flow channel

E4 Benches or terraces intercept runoff and direct it to a local flow system ................................................ 90

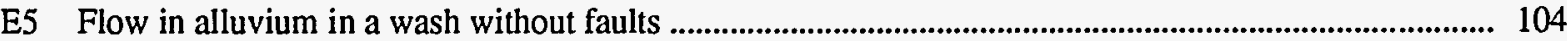

E6 Flow at bedrock contact in a wash without faults ......................................................................... 105

C3 Runoff intercepted by benches or terraces in a wash without faults .................................................... 106

A Runoff intercepted and diverted by exposed strata before reaching a wash ........................................... 110

A1 Runoff intercepted and diverted by exposed strata before reaching Solitario Canyon ............................. 111

A2 Intercepted runoff flowing in fractures to the Topopah Spring Units .................................................... 112

A3 Zone of perched water formed by rapid flow down a fracture ................................................................. 116

A4 Perched fluids interacting locally with the condensation cap .............................................................. 117

A5 Perched water acting as a continuing source of liquid at the vaporization isotherm ............................. 118

A6 Unsaturated and perched flow reaching the repository ................................................................................ 119

A9 Intercepted runoff being conveyed to the repository as fracture flow ..................................................

G6 Unsaturated-flow plume moving through the stress-altered region to reach waste containers and interacting with the condensation cap ............................................................................................................ 123

G7 Intercepted runoff carried by saturated fractures to the dryout zone ......................................................

G8 Intercepted runoff forming an unsaturated flow plume that interacts with the dryout zone ..................... 125

G9 Interaction of intercepted runoff that forms an unsaturated flow plume with the heat-pipe regime ........ 126

G11 Enhanced unsaturated flow plume reaching the stress-altered region .................................................... 128

A7 Intercepted runoff being converted to unsaturated flow ........................................................................ 129 


\section{LIST OF SKETCHES (concluded)}

Sketch

Page

A8 Distributed infiltration reaching the dryout zone .......................................................................... 130

G13 Intercepted runoff forming an unsaturated plume that interacts with the condensation cap ................... 136

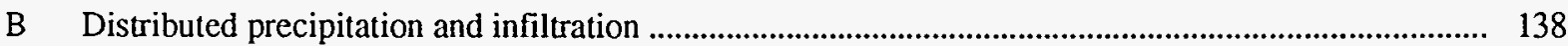

B1 Detail of surface distribution of rocks and soil, showing some infiltration .........................................

B2 Surface distribution of infiltration as affected by lithology ..............................................................

B3 Two-phase flow with venting of water vapor to the atmosphere .......................................................

B4 General view of mountain, repository, and heat-pipe regime .......................................................... 145

B5 Connected and partially connected fractures that are paths for perched flow to the repository ............. 147 


\section{LIST OF TREE SEGMENTS}

Tree Segment

Page

1 Nominal Flow, Runoff-Producing Events, Runoff to Washes, Washes With Faults, Surface-Channel Flow, Saturated Flow to Bedrock, Fracture Flow Through Bedrock

2 Nominal Flow, Runoff-Producing Events, Runoff to Washes, Washes With Faults, Surface-Channel Flow, Saturated Flow to Bedrock, Unsaturated Flow in Bedrock

3 Nominal Flow, Runoff-Producing Events, Runoff to Washes, Washes With Faults, Surface-Channel Flow, Saturated Flow to Bedrock, Saturated Flow in Faults

4 Nominal Flow, Runoff-Producing Events, Runoff to Washes, Washes With Faults, Surface-Channel Flow, Unsaturated Flow to Bedrock, Fracture Flow Through Bedrock

5 Nominal Flow, Runoff-Producing Events, Runoff to Washes, Washes With Faults, Surface-Channel Flow, Unsaturated Flow to Bedrock, Unsaturated Flow in Bedrock

6 Nominal Flow, Runoff-Producing Events, Runoff to Washes, Washes With Faults, Surface-Channel Flow, Unsaturated Flow to Bedrock, Saturated Flow in Faults

7 Nominal Flow, Runoff-Producing Events, Runoff to Washes, Washes With Faults, Subsurface Flow, Flow at Bedrock-Alluvium Contact

8 Nominal Flow, Runoff-Producing Events, Runoff to Washes, Washes With Faults, Intercepting Terraces, Saturated Flow to Bedrock, Fracture Flow Through Bedrock

9 Nominal Flow, Runoff-Producing Events, Runoff to Washes, Washes With Faults, Intercepting Terraces, Saturated Flow to Bedrock, Unsaturated Flow in Bedrock

10 Nominal Flow, Runoff-Producing Events, Runoff to Washes, Washes With Faults. Intercepting Terraces, Saturated Flow to Bedrock, Saturated Flow in Faults

$11^{\prime} \quad$ Nominal Flow, Runoff-Producing Events, Runoff to Washes, Washes Without Faults, SurfaceChannel Flow, Saturated Flow to Bedrock, Fracture Flow Through Bedrock

12 Nominal Flow, Runoff-Producing Events, Runoff to Washes, Washes Without Faults, SurfaceChannel Flow, Saturated Flow to Bedrock, Unsaturated Flow in Bedrock

13 Nominal Flow, Runoff-Producing Events, Runoff Intercepted, Flow Intercepted by Exposed Strata, Fracture Flow to Topopah Spring Units, Perched Flow

14 Nominal Flow, Runoff-Producing Events, Runoff Intercepted, Flow Intercepted by Exposed Strata, Fracture Flow to Topopah Spring Units, Matrix Imbibition

15 Nominal Flow, Runoff-Producing Events, Runoff Intercepted, Flow Intercepted by Exposed Strata, Flow into Shallow Fractures, Locally Saturated Flow

16 Nominal Flow, Runoff-Producing Events, Runoff Intercepted, Flow Intercepted by Exposed Strata, Flow into Shallow Fractures, Unsaturated Flow Plume

17 Nominal Flow, Distributed Infiltration, Fracture Flow, Shallow Fractures, Imbibition to Matrix 


\section{LIST OF TREE SEGMENTS (concluded)}

Tree Segment

Page

18 Nominal Flow, Distributed Infiltration, Fracture Flow, Shallow Fractures, Perched Flow, Locally Saturated Flow to Repository

19 Nominal Flow, Distributed Infiltration, Areal Infiltration, Matrix Imbibition, Unsaturated Flow Plume

20 Nominal Flow, Distributed Infiltration, Areal Infiltration, Perched Flow, Locally Saturated Flow to

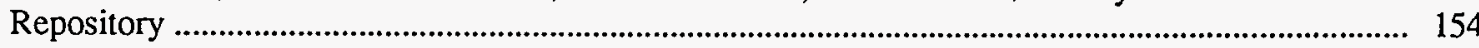

$21 \quad$ Nominal Flow, No Net Infiltration, Equilibrated Flow System .......................................................

22 Nominal Flow, No Net Infiltration, Draining Flow System, Locally Saturated Flow ......................... 162

23 Nominal Flow, No Net Infiltration, Draining Flow System, Unsaturated Flow ..................................... 164 


\section{LIST OF TABLES}

Table

1 Symbols Used in This Report ..
Page

-xiii- 


\section{SUMMARY}

The nominal flow system is that ground-water flow system that would exist at and around Yucca Mountain with the repository and heat-generating waste in place. It represents the expected case--the expected future of the repository. We cannot yet choose a single description or even a small set of future descriptions, because that would require the results of site characterization and experimental studies not yet available. In consequence, a large number of possible behaviors must be considered pending the acquisition of data or selection of a final repository design. These possible behaviors are described in an event tree, whose branches constitute scenarios. A series of sketches more completely explains the component elements of the scenarios; each scenario is defined by a connected path through the event tree and its associated sketches.

Future descriptions of the evolution of the mountain and the repository will require knowledge and understanding of probable climatic change and thermo-mechanical response of the mountain and repository system. Climatic change alters the boundary conditions for the mountain. We have chosen to construct scenarios in this report without reference to climate change specifically because we expect the effects of climate change to be automatically included in the modeling by specification of boundary conditions at the surface and other boundaries of whatever region is modeled. The thermo-mechanical response of the mountain is derived from the thermal output, distribution of heat-generating waste containers, and thermal and mechanical properties of the rock. Thermal expansion causes regions of compression close to the repository and regions of tension away from the heat source. The expected result is opening and closing of fractures. Fractures may open at the surface and be distributed through the mountain depending on overburden and existing stress state. We expect that any modeling will take into account the thermo-mechanical state of the mountain. The scenarios in this report must address a number of concerns, including

1) Where precipitation occurs,

2) Whether or not precipitation implies infiltration, that is, whether water enters the mountain,

3) How and when water in the mountain interacts with the repository-an interaction that can occur at a substantial distance from the waste,

4) How containers fail,

5) How (non-gaseous) waste is mobilized,

6) How contaminants are transported through the unsaturated zone below the repository, and

7) How contaminants are transported in the saturated zone to the accessible environment.

To describe these concerns diagrammatically, we have constructed a primitive decision tree designed to illustrate simply where analyses of features, events, and processes (FEPs) are necessary to resolve how the repository and mountain perform (Figure 1). The decision tree is organized as a series of yes-or-no questions. This is an oversimplification: for processes that can be lumped in this manner, each answer has a probability and an 


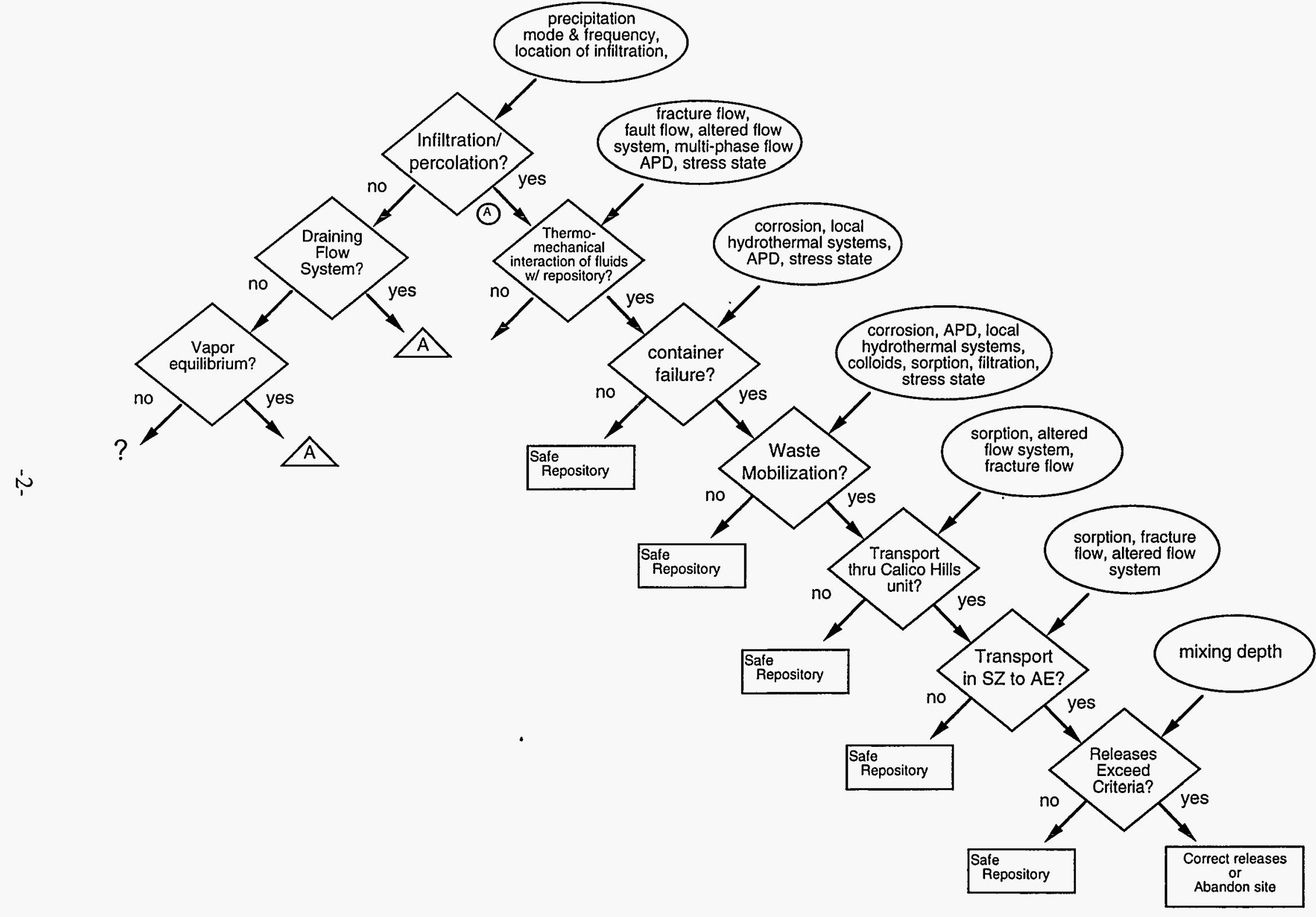

Figure 1. Decision tree illustrating where analyses of features, events, and processes are necessary to resolve how the repository system and mountain perform. $\mathrm{APD}=$ average power density: $\mathrm{SZ}=$ saturated zone: $\mathrm{AE}=$ accessible environment. 
uncertainty. In such a simple tree the lumping of features, events, and processes is extreme. The expansion of these lumped and idealized descriptions provides the rich development of scenarios. All event trees in the text are expansions of this general decision tree. Whether the site performs adequately is the final decision in this tree. Although all answers are shown as absolute yes or no, in fact our understanding will probably always be inexact.

This decision tree starts by asking whether infiltration implies percolation. Yucca Mountain researchers have suggested that possibly no infiltration percolates downward into the mountain at this time. Perhaps the downward percolation of fluids results from draining of precipitation deposited during some past pluvial cycle; conversely, there may be no percolation of liquids at all, and a vapor-maintained equilibrium may exist instead. Those options are included in the decision tree. Conditionals (indicated on this tree as ovals containing a short list of features, events, and processes that must be considered to reach a decision) support the decision for any query. For example, the first conditional indicates that frequency of precipitation, its mode (rain, snow, thunderstorm, etc.), and where it infiltrates are important to whether the fluid infiltrates the mountain.

The next query asks about the thermo-mechanical effects of hot fluids interacting with the repository. Those fluids may have newly entered the mountain or could be in the mountain already. The conditional supporting the query involve such FEPs as fracture flow, multiphase flow, average power density, and stress state of the rock. Most of this conditional will not be called out in the generalized event trees, except where it overlaps the next query. Instead it is used to set the boundary and initial conditions for scenarios.

The third query addresses how containers might fail and what local features, events, and processes contribute to that failure. The supporting conditional includes corrosion mechanisms, average power density, local stress state, formation of local hydrothermal systems (heat pipes, condensation caps), repository layout, rock chemistry, etc. The temperature dependence of FEPs in the conditionals force a distinction to be made whether any part of the repository is hot (hot is defined here to mean that the vaporization isotherm is outside a waste container, and cold is otherwise).

The fourth query asks about waste mobilization from failed containers and the movement of such mobilized contaminants into the surrounding rock. The supporting conditional includes the average power density, chemistry associated with container decomposition products, local hydrothermal fields, stress state, partitioning of contaminants between colloids and solute, sorption, etc. Although this conditional is a fertile source of FEPs for construction of detailed scenarios, until now performance assessment has treated mobilization by assuming, with some guidance from chemists, what the bounds might be. The reason has been ignorance; there are too few experiments and calculations specific to the environment to identify the most important processes. It has been assumed both that it is unnecessary to actually model detailed processes and also that it is too difficult to get data at the level of detail necessary to support such modeling. In essence, an integrated and bounding estimate of behavior has been presumed to suffice. Whether this is true remains to be shown.

The fifth query asks about transport of the mobilized contaminants away from the repository and through the Calico Hills units to the water table. The Calico Hills units, which are unsaturated, have been assumed in the Site Characterization Plan (U.S. Department of Energy, 1988) to be the major barrier to transport. These two observations-that the Calico Hills units are an unsaturated zone and that they form a major barrier-mean that 
considerable experimentation and modeling are required for confident assessment of their behavior. The supporting conditional includes investigation of temperature dependence of sorption, chemical alteration to flow systems, fracture flow, matrix flow, matrix diffusion, etc.

The sixth query addresses transport of waste in the saturated zone from arrival at the water table until contaminants reach the accessible environment. The supporting conditional includes sorption, fracture flow, matrix flow, thermo-chemical alteration of the saturated zone, connection to the unsaturated zone, etc.

The last query is the objective of the decision tree: "Do releases exceed the regulatory criteria?" This query also has a supporting conditional dedicated to what the criteria are. The type of standard may influence the data necessary to provide an answer; in meeting a dose standard, for example, the mixing depth in the saturated zone may be an important datum because it measures dilution.

The queries selected here are not unique choices, and there is overlap among them. This decision tree is intended only as a guide to the general structure of the scenarios given here and for analyses of the scenarios. The scenarios are presented in the form of generalized event trees rather than decision trees. Event trees allow multiple branches, that is, multiple possibilities at each FEP. Branches below any FEP (see Figure 3, p. 16) can be regarded as having passed through an "or" gate. Although probabilities could be assigned to each new branch, that will not be done here. We know too little about what is important for release, and there are too many branches to consider without preliminary screening by principal investigators (PIs). A tree starts with a basic initiating event or process potentially responsible for release of contaminants from a repository. To this basic event or process are added, in sequence, physically possible events and processes that might promote release. A scenario is defined here as a single connected path through the event tree, beginning with the initiating event or process and ending with release of contaminants to the accessible environment. We have attempted to include sufficient detail to help analysts construct reasonable experimental and numerical models to be used in performance assessment. Such important distinctions as whether scenarios are exclusive or competing will be considered at some later time. 


\section{INTRODUCTION}

Based on the performance-assessment method set forth in the Site Characterization Plan (U.S. Department of Energy, 1988), a set of scenarios encompassing all significant radionuclide-release paths from a nuclear-waste repository to the accessible environment must be described. Nominal flow of ground water has been identified as a process likely to eventually initiate a release of radionuclides from the potential high-level-waste repository at Yucca Mountain. The nominal flow system at the Yucca Mountain site is defined in this report to be the fluid flow system that includes entrance of fluids into and flow through the mountain in the presence of a repository for a varying climate and excludes disruptive events like tectonism or volcanism. This report attempts to catalog the details of the interactions between the features, events, and processes (FEPs) produced by ground-water flow in the presence of a repository system, the engineered barrier system (EBS), and waste. This catalog is developed in the form of scenarios describing the release of waste. If there were no failure of the waste containers and the EBS, there would be no releases, but it is presumed that they will corrode and eventually fail no matter how they are designed. To construct a complete and exhaustive catalog of release scenarios requires a synthesis of all currently available intormation on the geologic and hydrogeologic systems at Yucca Mountain and on the engineered components, i.e., the repository, EBS, and waste. Completeness in this sense is not possible; however, we do attempt to include all the physical principles and information known to us that we presume to have potential to contribute to significant radionuclide release. If additional release scenarios later become of concern, we will explore them in subsequent reports.

A number of scenarios are developed here for infiltration of precipitation into Yucca Mountain and interaction of that infiltrating liquid with the repository. An event tree constructed with the help of other project investigators is used to guide the construction. (Strictly speaking, the nominal flow system involves not just events but also hydrogeologic features and various processes, so event tree is a generalized term. The term FEP diagram is also used.) This tree is used to organize systematically the understanding that various investigators have reached of the hydrogeologic system, repository interactions, and associated phenomena. A "scenario" is defined in this report to be a connected path through the event tree, starting from the initiating event and continuing down to the release, with the requirement that the component elements in the path are well enough defined so that a well-posed, calculable problem results. We provide a series of sketches to more completely explain the component elements; each scenario is defined by a path and its associated sketches. Segments of the tree showing one or more paths will be discussed in detail until the tree is exhausted. The important issues of exclusivity and competing processes are not addressed by this definition. These issues will be addressed at some later date when the number of scenarios has been reduced.

Identification of a scenario presumes no knowledge of its probability of occurrence; rather a scenario represents a description of physically possible connected FEPs leading to a possible release of radionuclides to the accessible environment. Elimination of FEPs of little consequence, FEPs of very low probability of occurrence, and those that are, contrary to our current assumptions, physically impossible will be based upon observations, 
calculations, and experiments. We assume that the vast majority of the scenarios will eventually be eliminated, but by describing every scenario we have considered, we allow the reader to judge independently whether the work is complete and why scenarios are omitted in later stages of the analysis. We expect that the syntheses that produced these scenarios will induce principal investigators to suggest revisions and omissions of both scenarios and FEPs.

We expect frequent revision of the catalog of scenarios, and in fact this catalog itself represents a substantial revision and enlargement of earlier ground-water flow scenarios for a Yucca Mountain repository (Hunter et al., 1983; Ross, 1987). A number of times seem especially opportune for a catalog of scenarios to be reexamined and revised: when site characterization information from surface-based testing is available, when entrance to the underground workings is possible and incorrect presumptions about the behavior of the potential site can be corrected, to prepare for a possible construction license, when the Safety Analysis Report is required, and finally, if it is possible to construct and operate a repository, at decommissioning, when 30 to 50 years of operational experience will have been accumulated. That experience will allow further elimination of unimportant processes.

Although there are other definitions of "scenario," ours requires sufficient detail that if an alternative definition is required for any purpose, these results can be restructured to the required form. In addition, this definition parallels how workers lay out their experimental or calculational plans, so communication is relatively easy and our colleagues have little difficulty discovering our errors, omissions, or misinterpretation of their data or results. Scenario development is an iterative process that depends on feedback from our colleagues. Many scenarios constructed here will be considerably altered in the process.

A number of concerns must be addressed in all the scenarios constructed here:

A. Where precipitation occurs,

B. Whether there is runoff,

C. Whether there is infiltration to depth,

D. How fluids are directed to the repository (fluids include water vapor, water, water with various solutes, gases, and colloids),

E. How fluids interact with the repository,

1. With respect to elevated temperatures,

2. With respect to two-phase flow,

3. With respect to the stress-altered region around drifts and containers,

F. How fluids leave the repository, and

G. How the engineered barrier system corrodes or degrades (the EBS considered is the reference design of the Site Characterization Plan Conceptual Design Report, MacDougall et al., 1987).

To address these concerns, we developed the event tree with the following general characteristics. It distinguishes between runoff-producing precipitation that results in localized infiltration and distributed precipitation that results in widespread infiltration. It tries to distinguish among possible modes of infiltration and to consider structural features identified by the field research. It considers the possibility that new precipitation is not currently participating in the ground-water hydrologic system except at very shallow depths, i.e., that evaporation and 
transpiration remove all precipitation from the ground before it can percolate to great depth. Thus the tree for "Nominal Flow" has three primary branches. Two branches assume that at least some of the precipitation permanently infiltrates and can affect the ground-water system. A third major branch assumes that any infiltration is temporary and that evaporation or transpiration removes all new precipitation from the system. All scenarios in this report end when contaminants (waste) reach the water table. The scenarios focus on the coupled processes occurring in the unsaturated units of the mountain because of the repository that affect the flow system and thus the release of waste. (In this report, the word "unit" can describe geologic, hydrologic, or thermomechanical properties. We do not explicitly distinguish among these possibilities. The reader should consult the Yucca Mountain Project database for any particular use.)

Several circumstances influence the mode of infiltration and of percolation. The surface at which infiltration occurs is so far from the repository $(300 \mathrm{~m}$ ) that, except for vapor flow, it is not obvious that infiltration is affected by the mechanical and thermal effects of the repository. The areal and temporal extents of such effects are still open issues. Percolation, that is, movement of fluids down from the infiltration surface, is eventually affected by the mechanical and thermal effects. We include these coupled interactions of repository and fluid movement in the scenarios. Transit times for fluid movement depend strongly on the rock unit being traversed. Not all units are continuous over the mountain or have unvarying properties where they are continuous. For example, the Pah Canyon and Yucca Mountain units pinch out midway (from north to south) in the repository block. Washes, which dissect the mountain on the eastern slopes, are in some circumstances associated with faults.

Past calculations have assumed a uniform percolation at the repository horizon (Sinnock et al., 1986; Barnard and Dockery, 1990). This assumption allows thermal effects and releases to be calculated without knowing details of infiltration, which are not yet available. The assumption is equivalent to the presumption that, whatever the infiltration event and the percolation process, the characteristics of the mountain above the repository are sufficient to damp out any transient effects and to spatially integrate the influx-not unreasonable assumptions. Past calculations (Barnard et al., 1992; Wilson et al., 1994) have assumed that perched-water flow systems can bring locally saturated flow to the repository (weeps). Such systems are seen at Rainier Mesa (Wang et al., 1993). The assumptions for these two kinds of calculations are de facto reference scenarios and are special cases of scenarios to be discussed below in Section 3.2, Areal Infiltration. The paths, as all the paths discussed here, start with the premise of an episodic precipitation, infiltration, and percolation; however, a steady-state flux or a steady-state perched system at the repository is a possible result of the mountain's damping of influx. The de facto reference scenarios are simply special steady-state scenarios. They are not guaranteed to occur.

Certain precipitation events put water on the local infiltration surface (the ground) at a faster rate than it can percolate into the ground. Summer thunderstorms and rapid snow melt are two examples. Excess precipitation is redirected by runoff or underflow to other areas, where it may be imbibed by the mountain by various modes of infiltration. Thus the tree distinguishes between precipitation events that produce runoff and those that do not.

The discussion ends with arrival of contaminants at the water table, rather than arrival at the accessible environment as defined in Title 40, Part 191, Code of Federal Regulations, to allow us to focus on details unique to 
distinct scenarios and to avoid repetition of details of transport in the saturated zone. What is known about the saturated zone is discussed briefly in the last section.

A large body of literature describes the geologic and hydrogeologic setting of Yucca Mountain and vicinity (cf. Sinnock, 1982; Younker et al., 1992), and the Basin and Range province is currently an active research area with frequent new publications (e.g.. Duebendorfer and Black, 1992; Gomberg, 1991; Maldonado, 1990; Byers et al., 1989). Substantial further studies are planned or are in progress (U.S. Department of Energy, 1988). To review all new and existing work exhaustively is beyond the scope of this study. The intent of scenario studies is to include those interpretations and speculations relevant to the evaluation of possible repository success or failure. Stratigraphic nomenclature has been reviewed but does not conform with formal USGS nomenclature.

General topographic views of Yucca Mountain and the surrounding region appear in Figure 2. Figure 2a shows a view from the southeast looking northwest. Figure $2 b$ shows a view looking north up Solitario Canyon, which bounds the west side of Yucca Mountain at the repository.

\subsection{Conditions Imposed by the Repository}

The above discussion has been independent of the presence of the repository; the repository is deep and may not significantly affect the infiltration surface, except possibly by vapor-phase effects that will be considered separately. The presence of a repository imposes certain new physical conditions on the pre-existing flow system. Construction of underground openings causes durable alterations of the rock. A stress-altered zone around the drifts and emplacement holes develops in response to the change in stress when rock is removed. Strains develop to relieve this stress; typically the strains are radial and concentric fractures superimposed on any existing fracture systems (Jaeger and Cook, 1979). The mined openings will alter the stress state around the drifts-typically out to several opening diameters (Sketch G). Fluids must traverse the stress-altered region around each drift in order to reach the EBS (e.g.. Sketch G11,p. 129). This stress-altered region is an artifact of opening the drift and will persist for at least the repository life, whether or not the repository is cold (Blejwas, 1989). It is unclear whether this is favorable for repository performance. Most likely these fractures would decrease the connectivity of the rock matrix and make unsaturated flow to the drifts more difficult. The influence of stress alteration on fracture flow is not know. Alteration of hydraulic connections (e.g., by drainage of a controlling fracture) could establish completely new flow paths. Data on the effects of fractures will be collected by underground studies at Yucca Mountain.

In contrast to the effects of mechanical stress alteration, the effects of elevated temperature are not expected to be durable over the life of the facility. The thermal output of the waste gradually raises the rock temperature hundreds of feet away from the waste. Close to the waste, the temperatures may exceed those of water vaporization. Mechanical stress alteration is illustrated in Sketch G. Isotherms are not indicated (see MacDougall et al., 1987); they are transient features that change position with time. Calculations (Eaton and Reda, 1982; Doughty and Pruess, 1987; Eaton et al., 1987; MacDougall et al., 1987; Zhou et al., 1990) indicate that the local effect of the heat around waste containers is establishment of a heat pipe regime, followed by dryout with the possible formation of a 


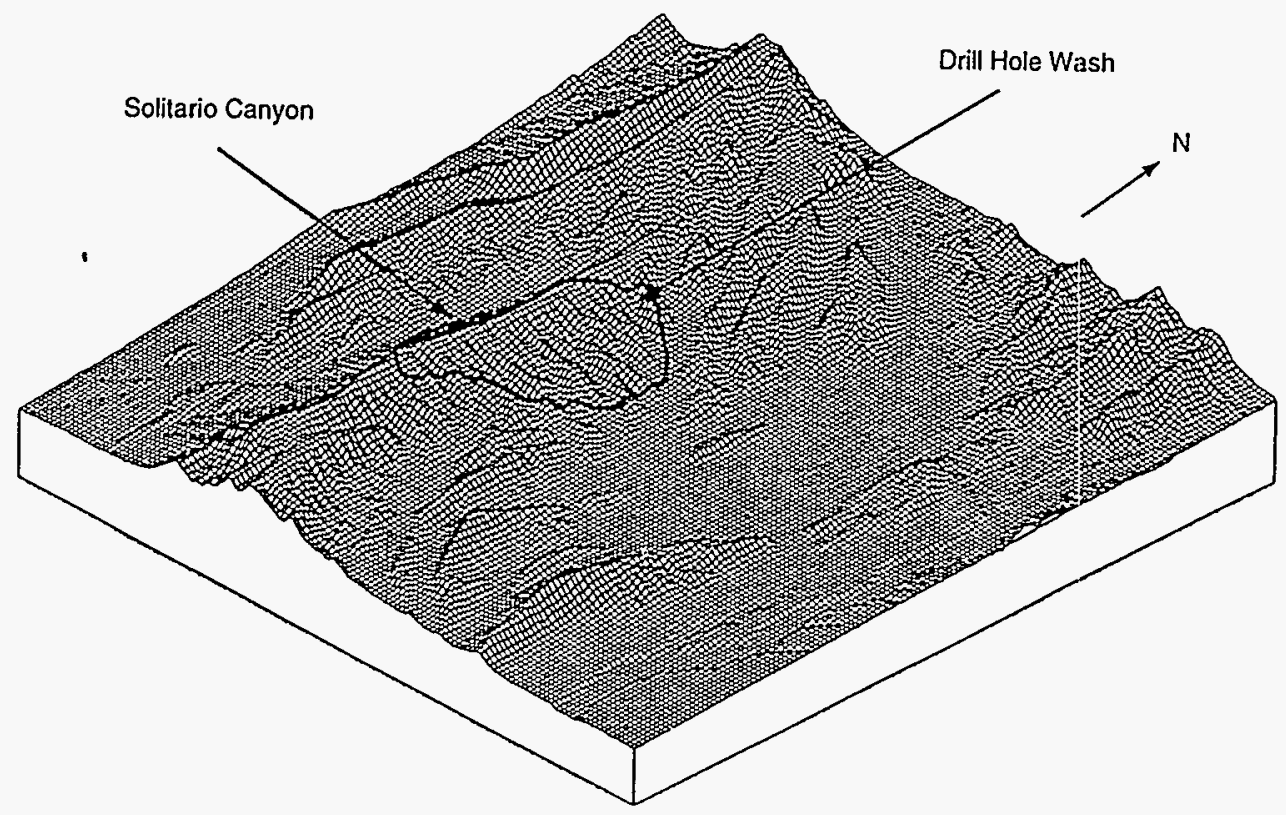

a. View from the southeast.

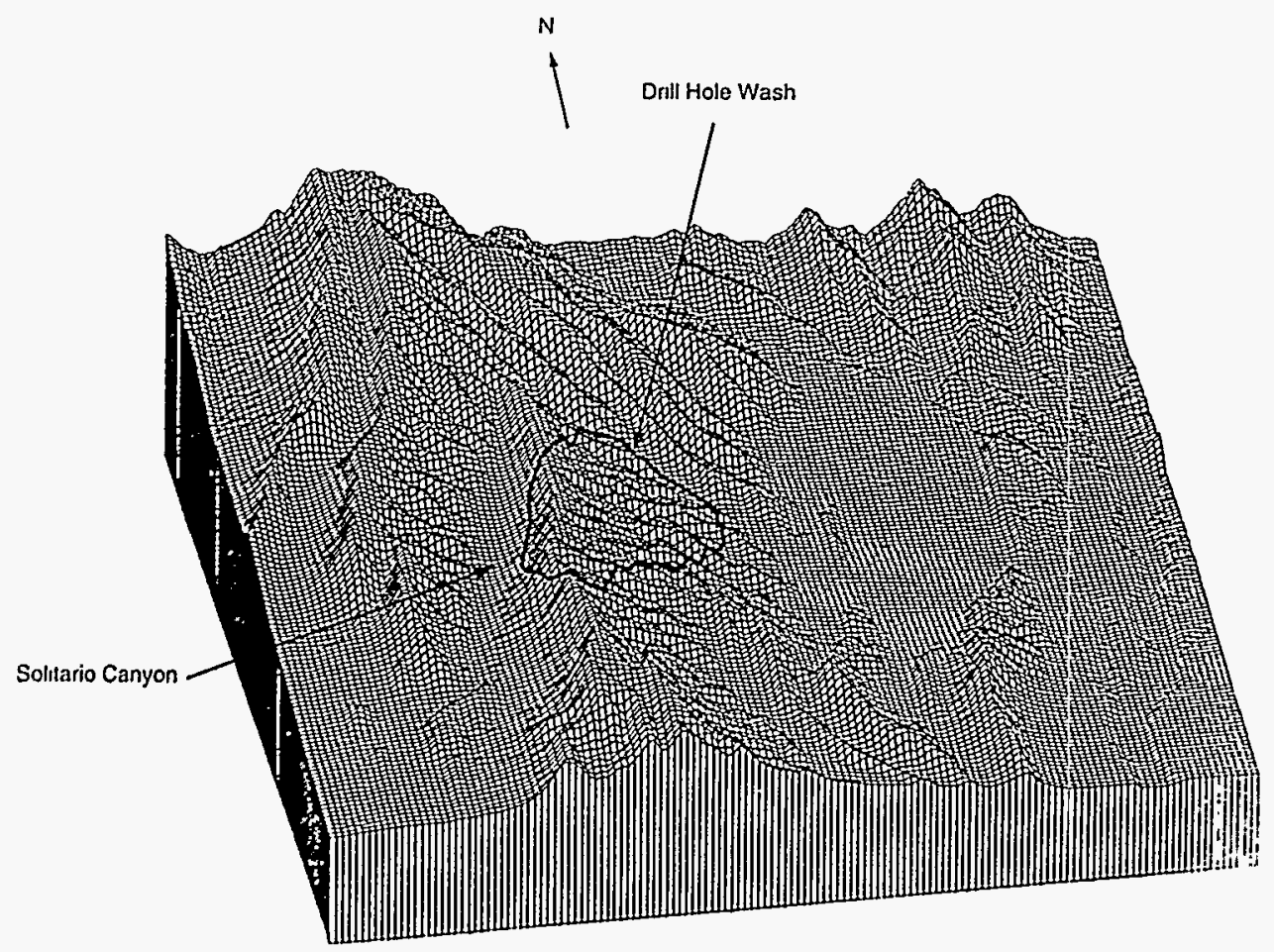

b. View from the mouth of Solitario Canyon.

Figure 2. Yucca Mountain. The dark line is a surface projection of the potential repository boundary. Drill Hole Wash roughly parallels the northern boundary of the repository. The east side of the mountain is dissected by washes; the west side is bounded by Solitario Canyon. These topographic features may influence infiltration of precipitation into the mountain. 


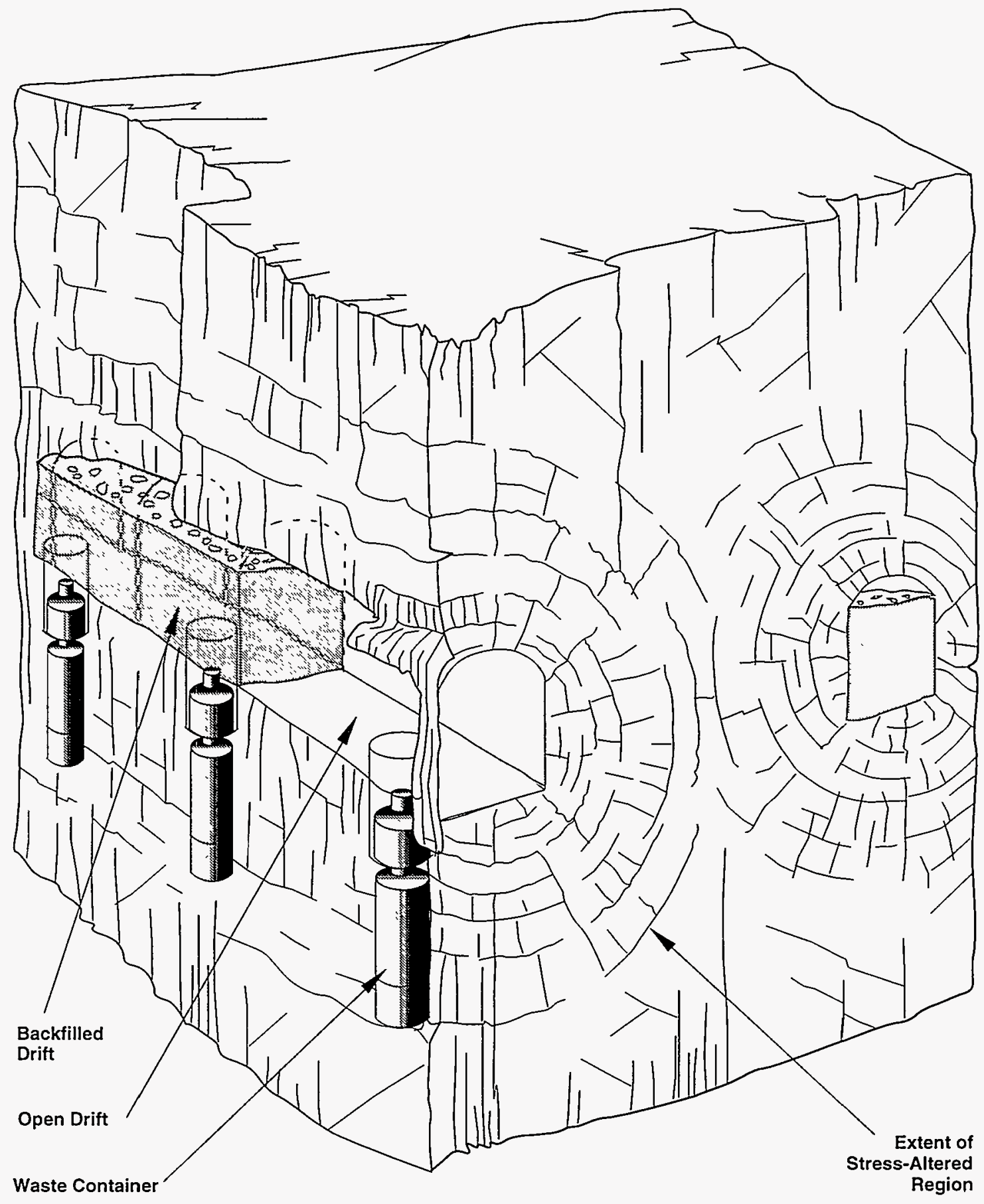

Sketch G. Near vicinity of repository, showing waste containers, drifts and backfills, and stress-induced radial and concentric fractures. 
condensation cap around the repository. It remains to be seen whether the water table acts as a continuous source of water and water vapor for this two-phase flow system. If fracture connectivity and matrix permeability in the rock above the repository are high enough, water vapor could be vented at the surface rather than continue to circulate in a two-phase convective system. Each of these possibilities will be considered in turn in the scenarios developed here.

Two additional aspects of the temperature field must be considered. First, as the temperature rises and the rock expands and dries, the solubility of minerals changes. When the rock is dry, all dissolved solids precipitate, and plugging of pores and fractures is possible. Second, certain flow parameters are functions of temperature. Volume expansivity of water is roughly $1 \%$ over the range ambient $\left(28^{\circ} \mathrm{C}\right)$ to boiling $\left(97^{\circ} \mathrm{C}\right)$, and viscosity changes by almost a factor of 3 over the same range. This suggests that rapid flow from the heated region may cause saturation of a zone below the repository with condensate; however, the source of fluids for this saturated zone differs from that used by earlier two-phase calculations (Sketch G1). A substantial number of studies and simulations of natural geothermal systems and of idealized repositories in tuff provide a broad basis of understanding (Buscheck and Nitao, 1988; Nitao, 1988; Pruess et al., 1990; Grant et al., 1982).

\subsubsection{Movement of Vaporization Isotherm}

A hot waste container emplaced in a repository put into an emplacement hole immediately begins heating the surrounding rock by convection in the surrounding air gap, by radiation across the air gap, and by conduction at any points of physical contact across the gap (cf. Ramspott, 1991).

The conceptual model, based primarily on calculations, suggests that heating causes the vaporization isotherm to move away from the waste container and out some distance into the surrounding rock. In a year or two, isotherms from adjacent containers in a row of waste containers will coalesce and the vaporization isotherm can be represented by a long oblate cylinder roughly centered along the waste containers (Eaton and Reda, 1982; Eaton et al., 1987). After a longer time, vaporization isotherms from adjacent rows of containers will coalesce, forming a roughly oblate spheroid around the repository. This movement of the vaporization isotherm is accompanied by two-phase movement of the fluid and possibly by drying of the rock. As the radiogenic heat source decays, the temperature at the waste containers decreases and the vaporization isotherm slowly collapses back to the containers. The isotherm reverses its development, although the details of its behavior may not exactly reverse the path followed during development, because of hysteresis. This description is idealized because not all the waste containers will have the same thermal output and not all will be loaded into the repository at the same instant. Spatially, the container placement density may vary due to stand-off areas required by fault zones or actively weeping fractures. Therefore, expansion and contraction of the vaporization isotherm will be more complex in its local details than this simple description suggests. 


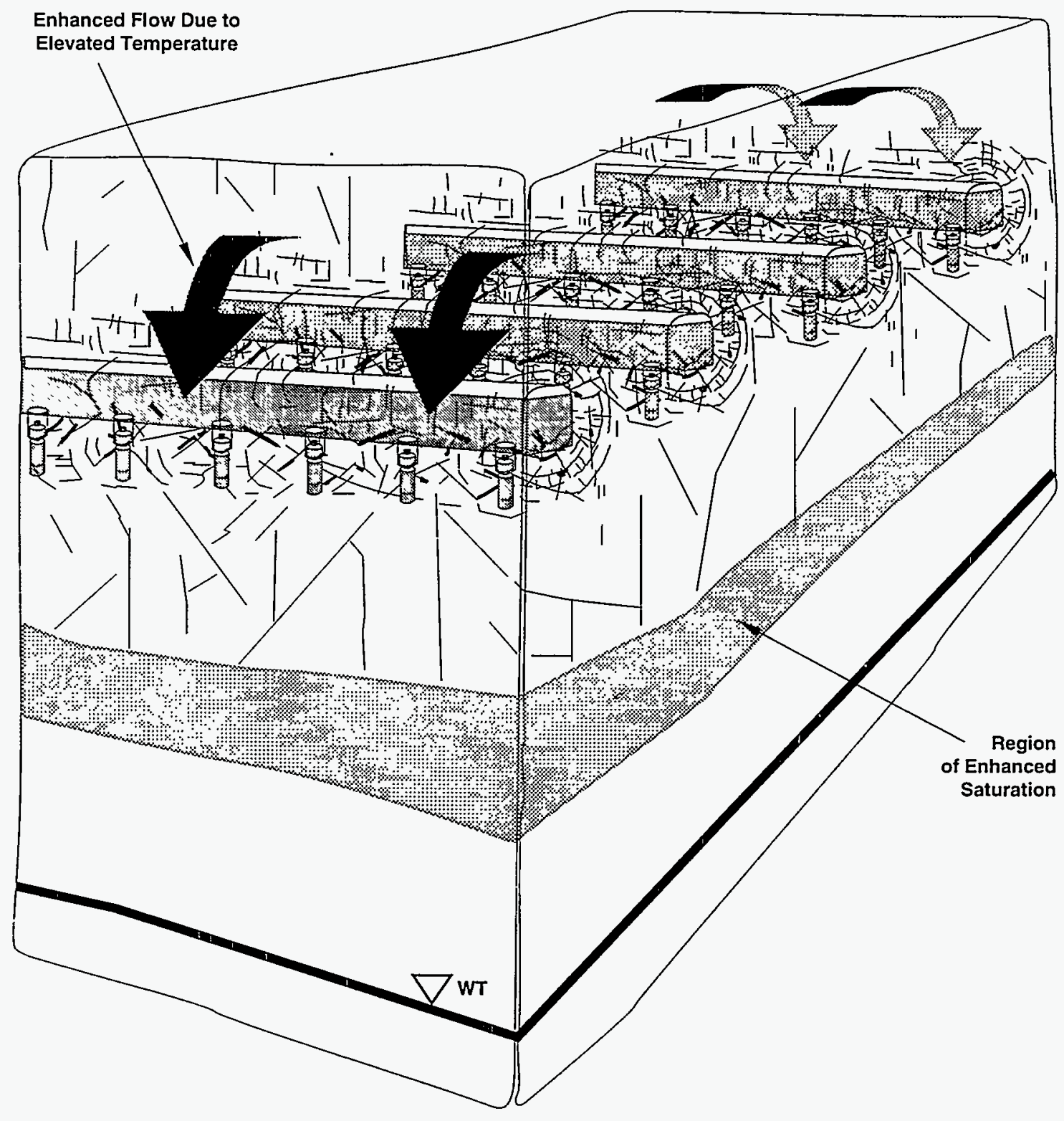

Sketch G1. Thermally induced rapid downward flow of water through the repository to form a semi-perched zone below. 


\subsubsection{Mine Ventilation}

The mining operation that constructs the emplacement drifts is ventilated. Ventilation can remove considerable moisture from the host rock and alter initial conditions for the emplaced waste containers. Numerical simulations of ventilation of an isothermal drift ( $6.7 \mathrm{~m}$ high and square in cross section) indicate that about the first two meters of rock wall dries at a water-removal rate of about $3.6 \times 10^{-6} \mathrm{kgm} / \mathrm{sec}$ per meter of drift (Hopkins et al., 1987). Hopkins et al. (1987) also examined drying for periods to 50 years, so the case of an emplacement drift not used immediately has also been considered. However, we presume here that once an emplacement drift has been driven, it will be used without delay.

When waste containers are emplaced, the thermal load immediately starts two-phase convective processes. These processes deliver additional moisture to the ventilation system. Pruess et al. (1988) and Pruess and Tsang (1991) estimated that about $7 \times 10^{-4} \mathrm{kgm} / \mathrm{sec}$ of moisture for each container is driven to the drift if an emplacement hole is well-connected to the drift. If containers are five meters apart, this translates to about $1.4 \times 10^{-4} \mathrm{kgm} / \mathrm{sec}$ per meter of drift or about 40 times the drying produced by isothermal ventilation alone. While the drift is open and ventilated and waste containers are well-connected to the drift, simulations indicate that as much as two-thirds of the total heat being generated could be removed by mine ventilation (Pruess and Tsang, 1991).

Once the drift is filled, the openings are sealed. (There are no further demands on the mine-ventilation system except for leakage around the seals.) Numerical simulations (Pruess and Tsang, 1991) for unventilated emplacement show that complete dryout occurs only within a few meters of waste containers and partial dryout in a region of perhaps 100 meters around the repository. These calculations used pre-emplacement initial conditions; further calculations are needed to cover the transition from ventilation to no ventilation. How much drying of the rock during ventilation must be considered in long-term analyses depends on how long emplacement drifts are ventilated. For the short time indicated earlier, with prompt sealing (Parsons, Brinckerhoff, Quade and Douglas, Inc., 1987), drift temperatures, i.e., rock-mass temperatures at the drift, may reach $120^{\circ} \mathrm{C}$ within 50 years (Wallace and Zerga. 1987). It is likely then that drying is so dominated by later thermal output of the waste that the initial drying during ventilation is only a minor alteration on conditions. In contrast, long-term ventilation, for example to repository closure or about 50 years, may remove two-thirds of the heat and substantial moisture. This removal of substantial heat and moisture would alter the initial conditions for long-term isolation.

\subsubsection{Condensation Cap}

Another effect of the repository might be the creation of a condensation cap. When the temperature close to the waste rises above the vaporization temperature, water vapor rises away from the heat source and condenses in a cooler region, forming a localized zone of saturation. For the rocks at Yucca Mountain, i.e., fractured porous tuff, and for an areally distributed heat source, formation of such a zone of condensation above the repository is conceivable. Calculations for idealized models (Pruess, 1986) show the existence of a condensation cap on a small 
scale. Recent modeling (Buscheck and Nitao, 1993) suggests the formation of a condensation cap on a large scale as well. Although corrosion and degradation of the waste container and waste may occur during the hot periodbecause of attack by water vapor and any included volatiles - transport away from the waste container requires the temperature to be below vaporization for the non-gaseous waste considered in this report. The return of fluid temporarily stored in the condensation cap to the repository must be considered. During the hot phase, vaporization may continuously alter permeability and porosity as fracture-filling materials are deposited or removed (indicated schematically in Sketch $\mathrm{I}$ ). The returning fluid is expected, because of the temperature distribution, to pass through this altered region. The extent of plugging of pores and fractures is unknown and can only be resolved through experiments. Any return flow of the condensate is expected to occur first through the rock between drifts and later around the edges of the repository. For some possible thermal loadings, the water table ( $\sim 200 \mathrm{~m}$ away) could serve as a continuous source of fluid for the convective cell. Once the rock and repository have cooled sufficiently to allow liquids to persist at waste containers, reflux from the condensation cap could act as a source of fluids. The reflux could occur as a saturated flow down fractures or it could combine matrix and fracture flow.

\subsection{Reading This Report}

This report is accompanied by a nominal-flow event tree, located in the back pocket. Frequent reference to this tree may be essential for following the logic of the discussion in the text. Figure 3 presents an overview of the event tree and provides a structural outline for this report.

Each scenario encompasses a series of FEPs. Specific FEPs are indicated in the text by quotation marks. Each FEP is discussed in detail in the text, illustrated by a sketch, and connected to preceding and subsequent FEPs in the event tree. Text, sketches, and tree segments are cross-referenced by means of sketch and tree-segment numbers. Each sketch illustrates one FEP discussed in the text and may be distorted in proportion, as a cartoon, to enhance features of interest that might not be discernible if drawn to scale.

Small parts of the event tree, called tree segments, are reproduced in the text to aid discussion. A tree segment is a diagram in the text containing a set of branches excerpted from the event tree. The ellipses in the lowest tier of Figure 3 map the tree segments into the complete event tree. For example, TSi refers to Tree Segment 1 in the text (p. 32) and to the left-most branch of "Washes With Faults" in the event tree (pocket). Each tree segment consists of two similar diagrams. Its caption traces the tree path from the initiating event ("Nominal Flow") to the FEP immediately preceding the branches for hot and cold repositories. For example, from Figure 3 and the complete event tree in the pocket, we see that Tree Segment 1 will have the caption "Nominal Flow," "Runoff-Producing Events," "Runoff to Washes," "Washes With Faults," "Surface-Channel Flow," "Saturated Flow to Bedrock," "Fracture Flow Through Bedrock." Each caption thus restates the assumptions upon which the discussion of the tree segment is based. Part "a" of the tree segment shows a portion of the event tree and directs the reader to the sketches associated with that portion. Part " $b$ " of the tree Segment shows by a broad arrow, scenarios or groups of scenarios discussed from that portion of the event tree. Each scenario is identified in both 


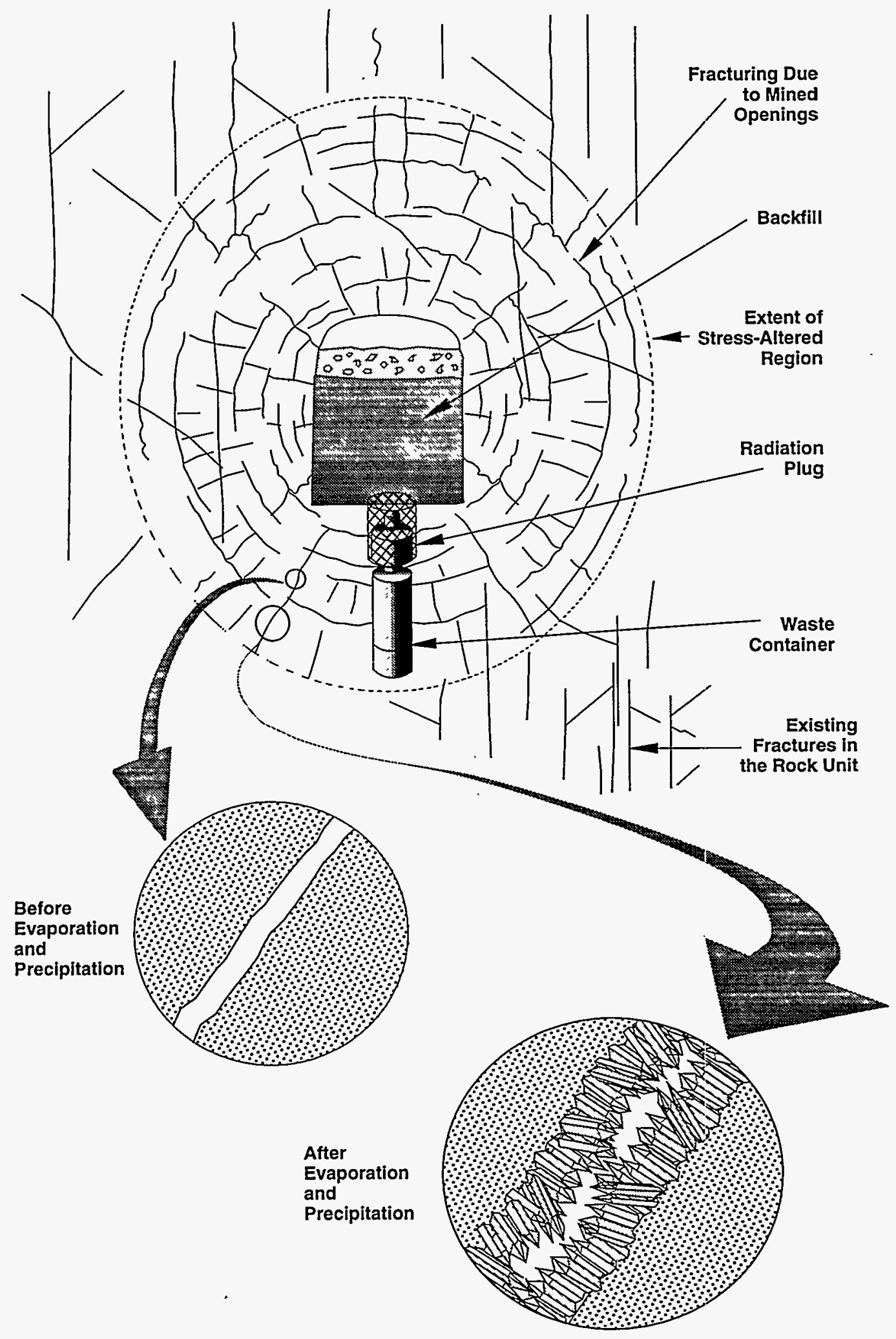

Sketch I. Diagrammatic cross section of waste container, drift and backfill, and induced and previously existing fractures. Fractures are three-dimensional and may intersect outside the plane of the sketch. The enlargements show the very-small-scale process of alteration of permeability and porosity by deposition of precipitates in pores and fractures when liquid is evaporated. 
tree segment and text with a numbered pentagon. For example, Path 2.1 in the text refers to the first path in Tree Segment 2b, ending in "Transport to Water Table," Sketch M3. Some sets of branches are common to more than tree segment. Each of these sets, when first described, is accompanied by a circled letter, e.g., (2). Rather than repeat the entire set of branches at each occurrence, the circled letter appears alone to indicate where the set is replicated. These symbols are summarized in Table 1.

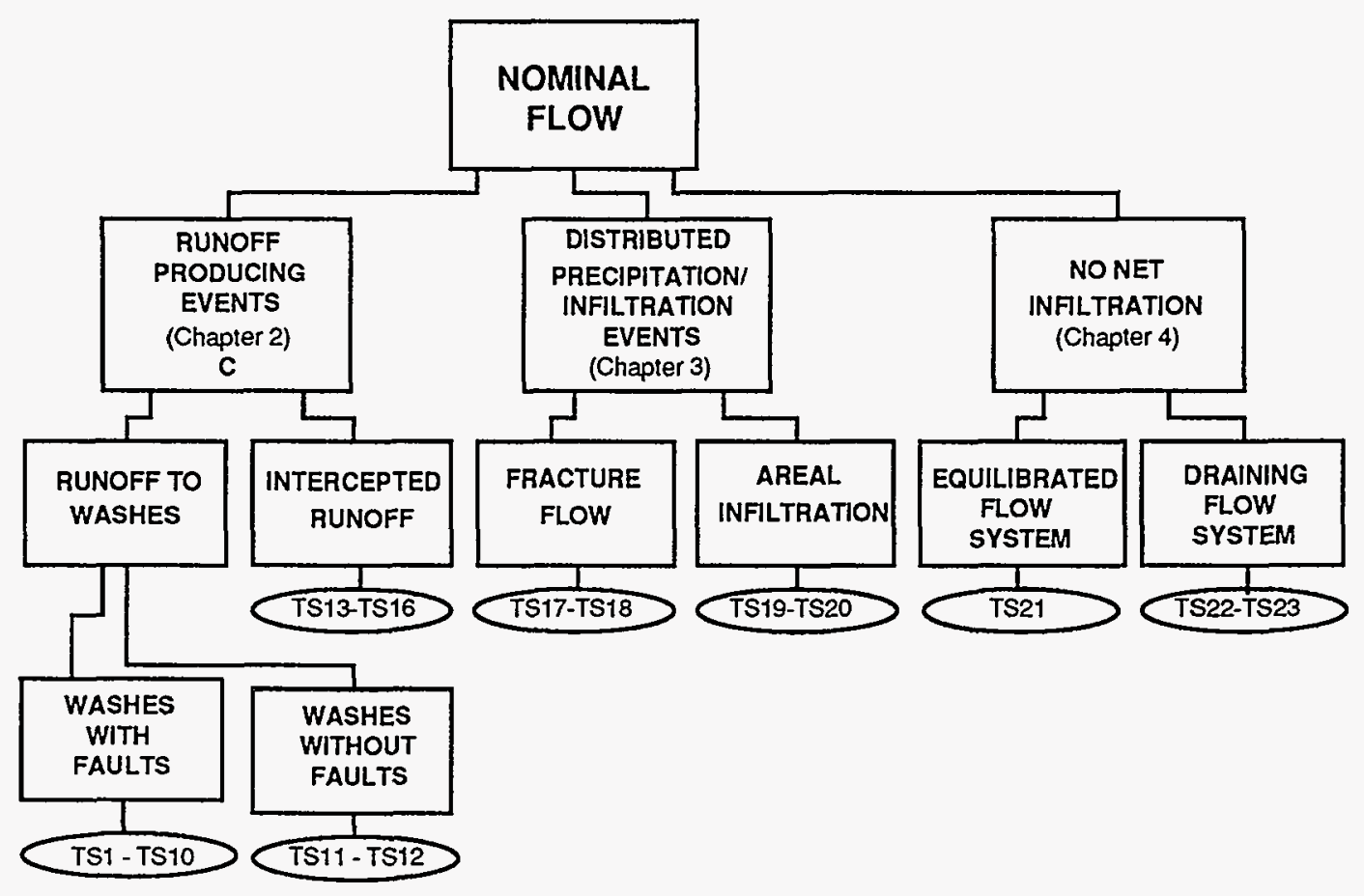

Figure 3. Overview of the event tree for nominal flow. 
Table 1. Symbols Used in This Report

\begin{tabular}{|c|l|}
\hline Symbol & \multicolumn{1}{c|}{ Usage of Symbol } \\
\hline \hline TSx & $\begin{array}{l}\text { Symbol for Tree Segment x showing its position in the event tree and } \\
\text { in this report. }\end{array}$ \\
\hline Symbol for a branch that is repeated elsewhere in the event tree. \\
\hline Z & Position of a repeated branch. \\
\hline 2.1 & $\begin{array}{l}\text { Symbol and reference number for a scenario path through } \\
\text { the event tree. }\end{array}$ \\
\hline
\end{tabular}




\section{RUNOFF-PRODUCING EVENTS}

The left-hand branch in Figure 3 (p. 16), "Runoff-Producing Events," will be discussed first. Runoffproducing events (Sketch $\mathrm{C}$ ) are differentiated into two fates for the precipitation—runoff to washes and runoff imbibed by the strata and soil over which it flows.

\subsection{Runoff to Washes}

Washes drain runoff from Yucca Mountain, particularly on the east side. Two different kinds of washes have been distinguished: those which are associated with faults and those which do not appear to be associated with faults (Figure 4, p. 23). This distinction is important because the presence of faults may affect infiltration. Runoff can leave the mountain on the surface, evaporate, or infiltrate. The size, shape, gradient, and so on of the washes control how much water runs off and how much infiltrates. This section discusses runoff that infiltrates.

Washes in roughly the north half of the block are underlain by the partially welded to non-welded Pah Canyon and the Yucca Mountain Members (Sketch C1). These two units, which appear to pinch out to the south about halfway along the block (Scott and Bonk, 1984; Frizzell and Shulters, 1990), are components of what has been informally designated the Paintbrush non-welded hydrologic unit (U.S. Department of Energy, 1988, Ch. 3). Over the northern half of the site the welded Tiva Canyon unit is separated from the densely welded Topopah Spring unit by a sequence of non-welded and bedded tuffs that are designated the Yucca Mountain Member and the Pah Canyon Member. The location of these units is important to modeling because their presence or absence affects the contrast of hydrologic properties between the welded and nonwelded units and thus the change from unsaturated to saturated flow and vice versa (Sketches $\mathrm{Cl}$ and $\mathrm{C} 2$ ).

\subsubsection{Washes With Faults}

Washes with faults differ from washes without faults in two respects. First, the fault zone itself may substantially control the hydraulic characteristics of the wash in which it occurs. Second, no waste will be emplaced in a recognizable fault zone where it passes through the repository. The general structure of a wash with faults is shown in Sketch D, which indicates runoff to an alluvium-filled wash with an active drainage and a stratigraphic offset caused by underlying faults.

Drill Hole Wash essentially forms the north boundary for the repository. It is a large wash with a considerable collection area and is apparently fault-controlled. It may be that Drill Hole Wash and other washes like it will eventually be shown to affect fluid flow to the repository. Fluid infiltrating in washes outside the boundary of the repository must migrate considerable distances laterally to reach the repository, and thus modeling of flow for these washes might differ slightly from modeling of flow for fault-controlled washes over the repository. We treat them all together, however. 


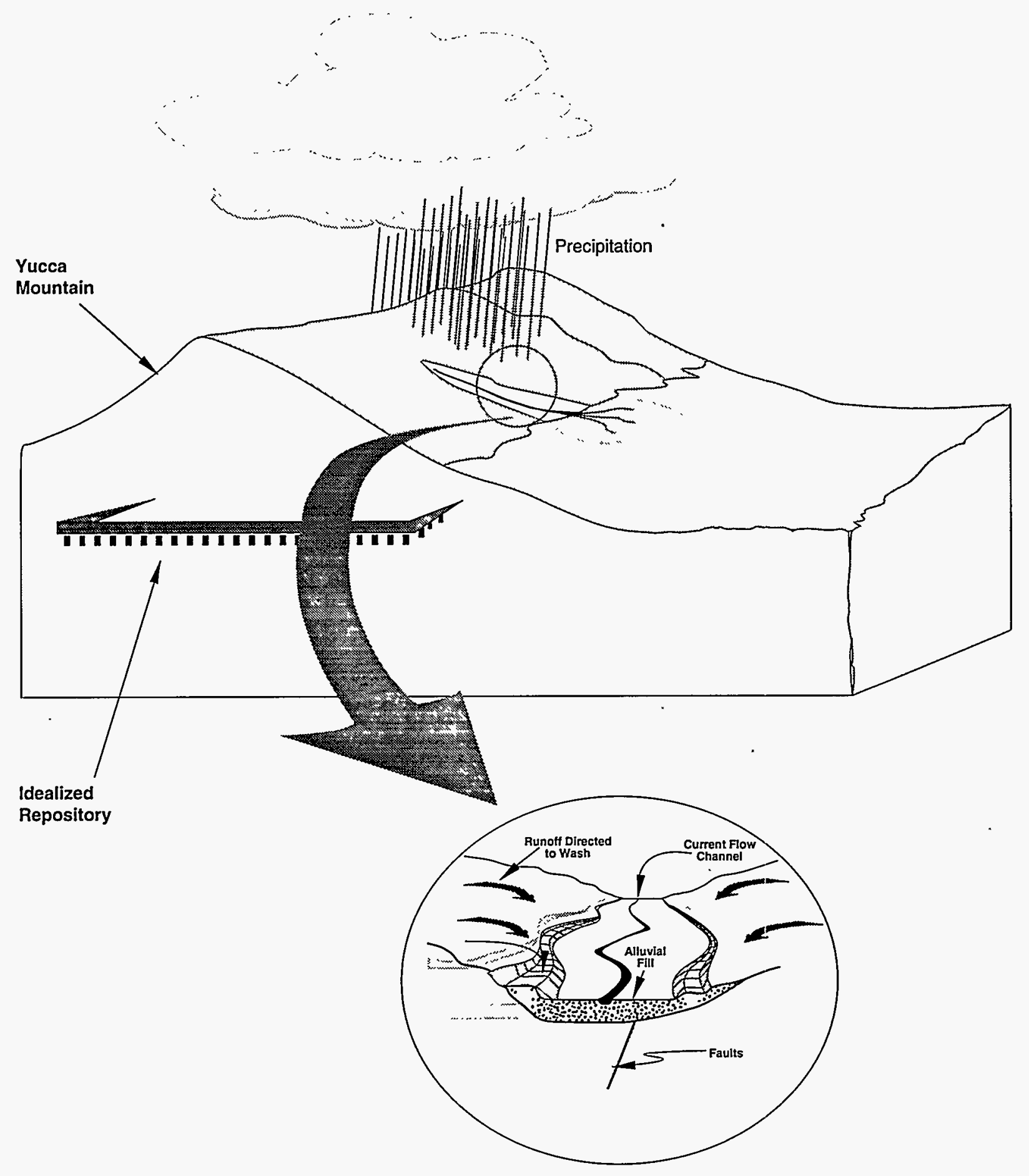

Sketch C. Runoff-producing events. Precipitation flows over the ground surface to washes and down the channel for some distance. 


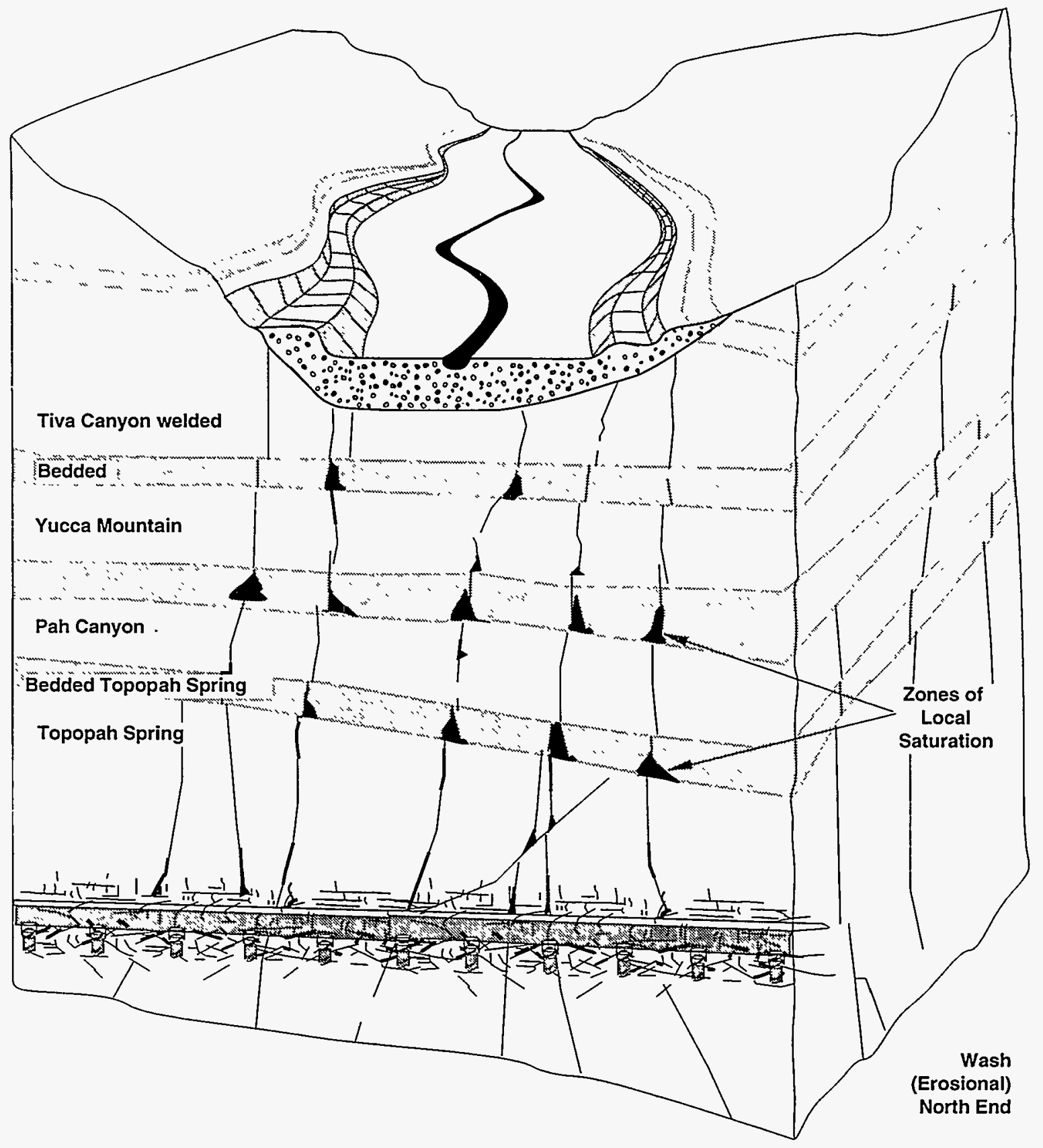

Sketch C1. Strata dependence of the mode of infiltration, i.e., saturated flow in fractures vs. unsaturated flow in matrix, for the stratigraphic units occurring at the north end of the repository. 


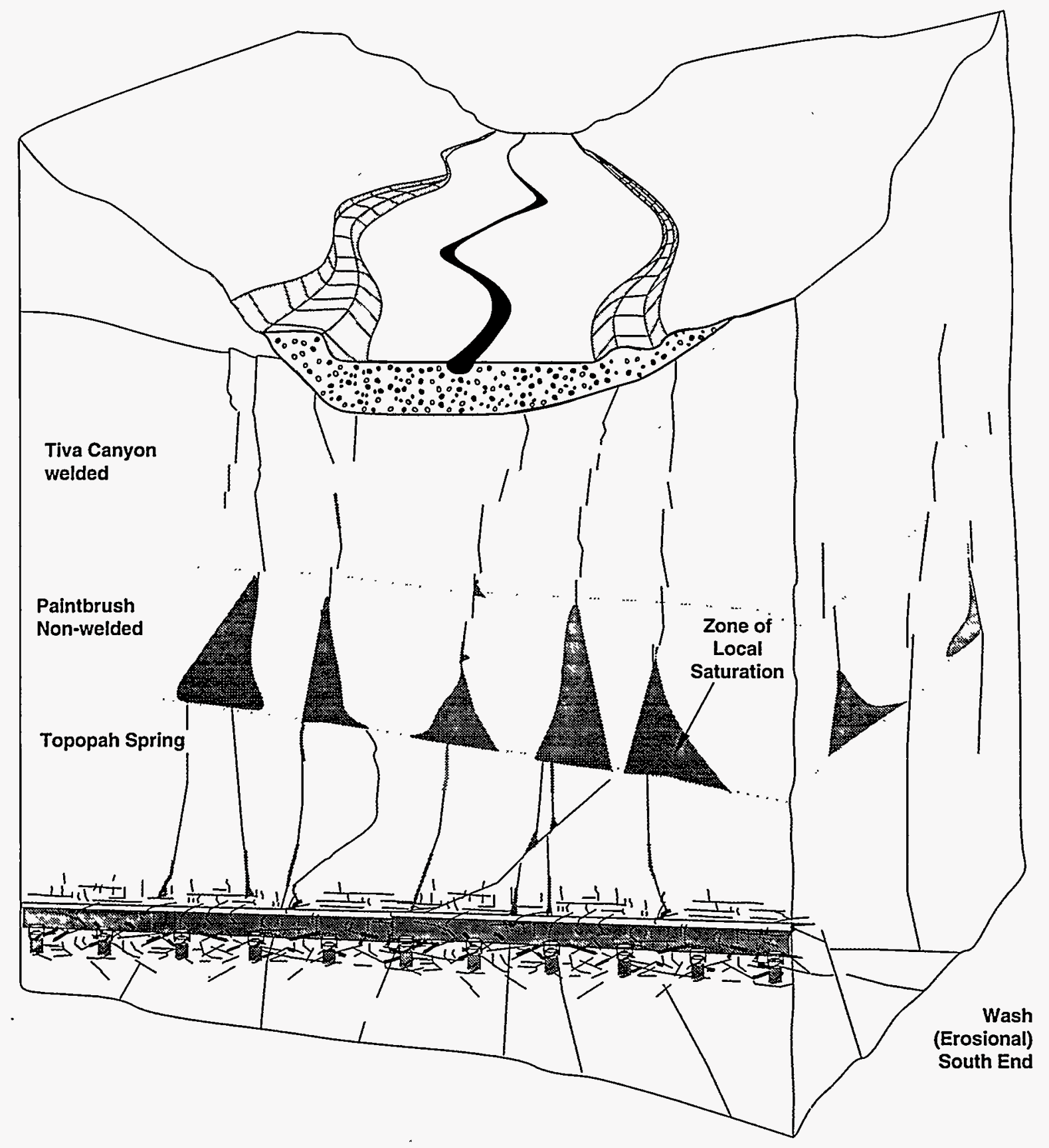

Sketch C2. Strata dependence of the mode of infiltration, i.e., saturated flow in fractures vs. unsaturated flow in matrix, for the stratigraphic units at the south end of the repository. 


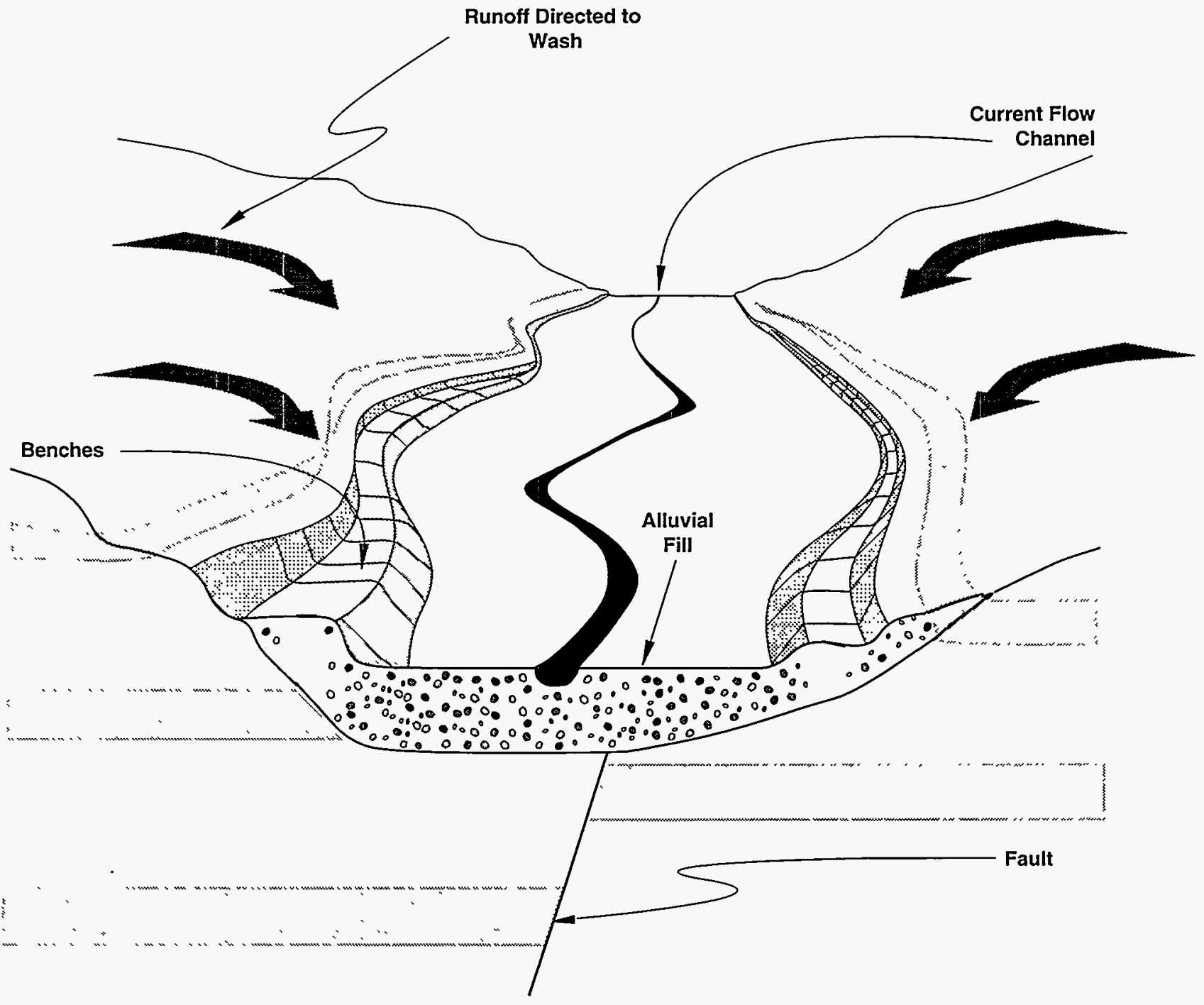

Sketch D. Surface-flow channel in a wash with faults. 


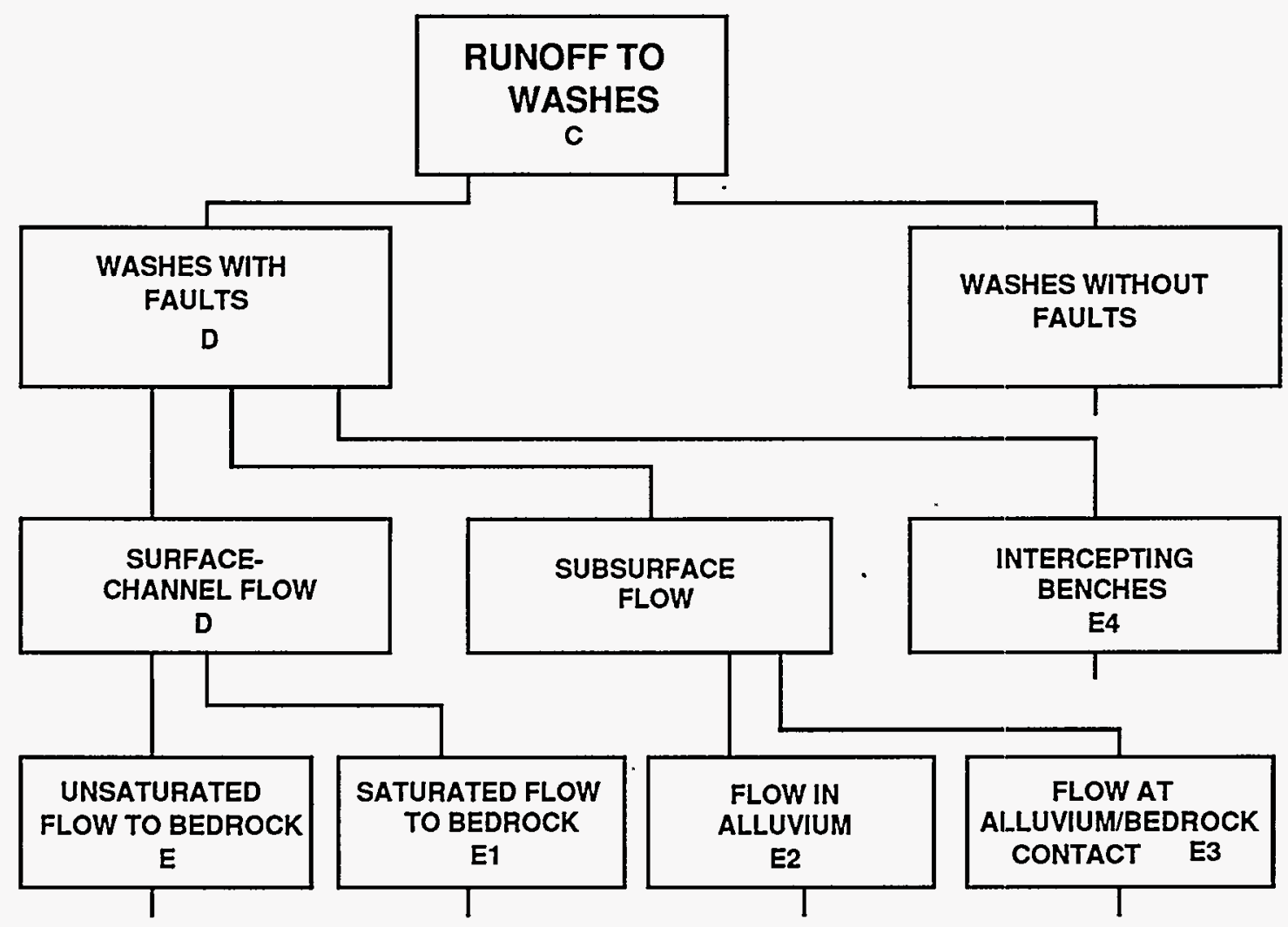

Figure 4. Nominal Flow, Runoff-Producing Events, Runoff to Washes.

Details of the lithology below the wash, e.g., whether the units are bedded or unwelded, will control how flow passes through the stratigraphic section. The Yucca Mountain and Pah Canyon Members are welded north of the site and become less welded southward. This change in welding may alter their ability to support fractures and thus affect the flow regime.

\subsubsection{Surface-Channel Flow}

The surface-flow channel acts as a collector for runoff, conducting water from areas of high runoff in the wash to other areas down the wash. The channel acts as a source for leakage into the alluvium. In some cases, leakage may occur in areas far removed from the original precipitation. We ignore water in the channel that moves down the wash and away from the mountain too far to affect the repository. Sketches E and E1 illustrate two possibilities for fluid movement from the channel through the alluvium to the bedrock and faults below. 


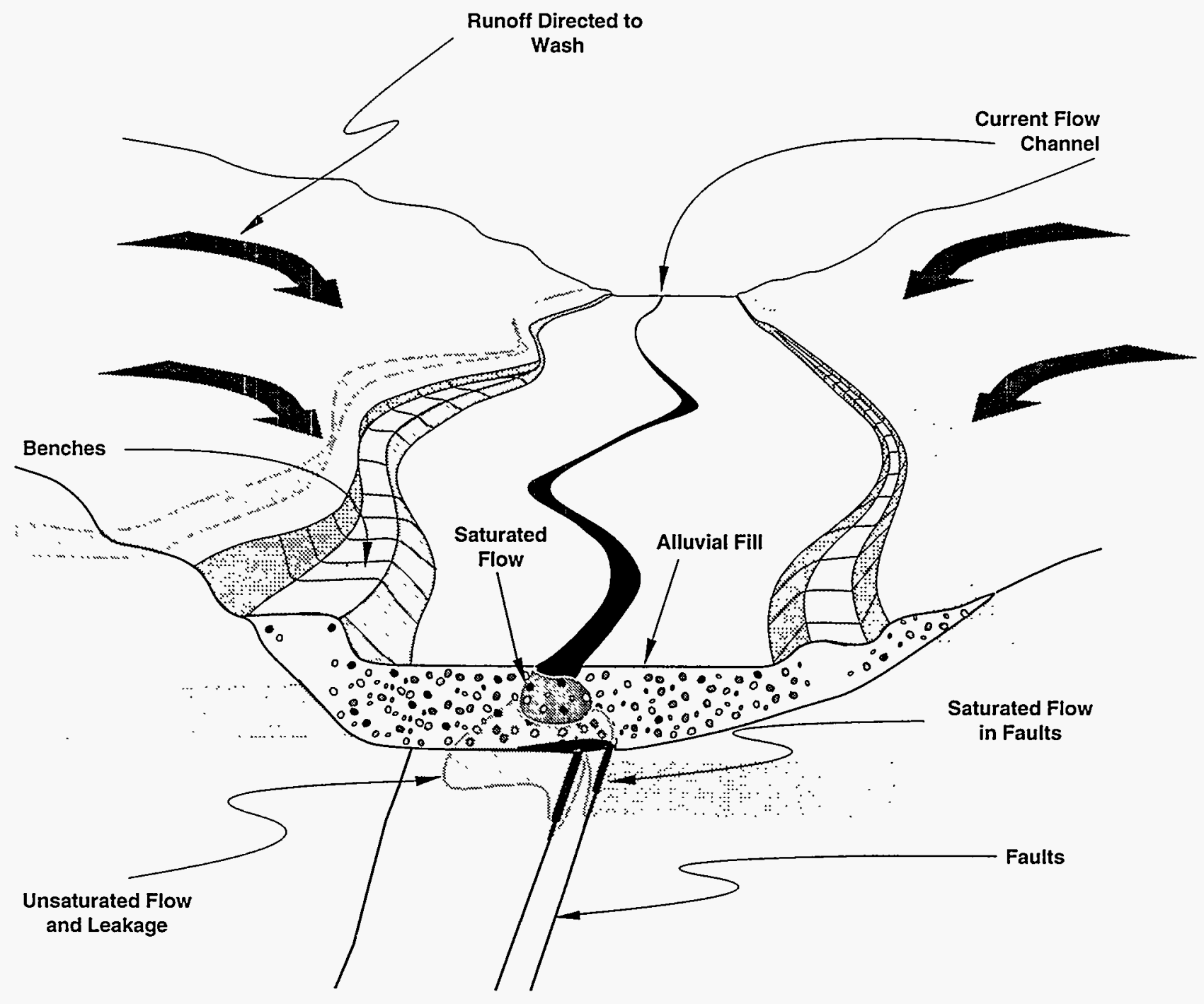

Sketch E. Small-scale diagrammatic cross section of a wash with faults, showing unsaturated flow through the alluvium. The flow system and the wash are three-dimensional, and the relative positions of the flow channel, bedrock, and areas of saturated and unsaturated flow may vary outside the plane of the sketch. 


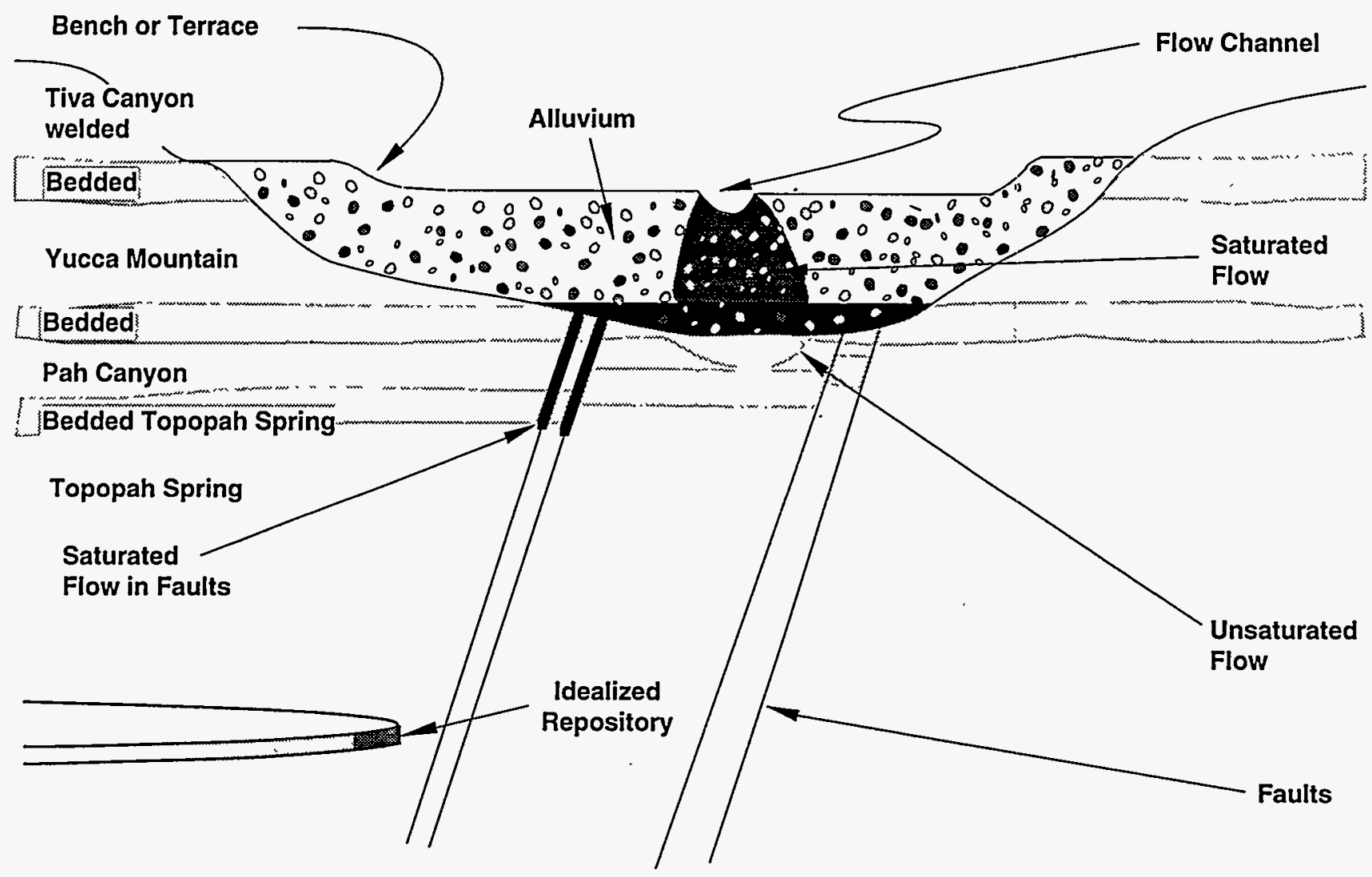

Sketch E1. Small-scale diagrammatic cross section of a wash with faults, showing saturated flow through the alluvium. 
The unsaturated-flow branch is illustrated in Sketch E. Runoff down the flow channel leaks from the channel and creates a small zone of saturated flow immediately adjacent to the channel, which in turn is enclosed by a larger zone of unsaturated flow in the underlying alluvium. Presumably the unsaturated flow column proceeding downgradient reaches bedrock at various places along the wash bottom. At bedrock flow may continue in several ways: as equilibrium fracture flow (described in and as a consequence of the composite-porosity model) (Peters and Klavetter, 1988), as unsaturated flow in the bedrock (supplied by leakage from the alluvium), and as flow down faults (gravity-driven flow). We recognize that the latter two cases of flow could be calculated directly using existing unsaturated/saturated-flow computer codes; however, we wish to emphasize the possible duality in behavior and therefore treat these flows as if they were independent.

Sketch E1 assumes a sufficiently persistent flow down the channel to sustain a saturated flow column from the channel down to bedrock. Although this column is ephemeral, ponding at the alluvium/bedrock contact causes intermittent saturated flow. The saturated alluvium provides a source for fracture flow through the bedrock (again by the composite model), for unsaturated flow in the bedrock, and of leakage to the faults.

If the transition from welded to nonwelded tuff in the Yucca Mountain and Pah Canyon Members occurs south of Drill Hole Wash, then Sketch E as well as Sketch E1 would continue in Sketches F1, F2, and F3. Sketch E shows leakage from the surface-flow channel establishing an unsaturated flow system through the alluvium to the bedrock and local ponding at the interface, which allows saturated flow down the faults or fractures or unsaturated flow into bedrock (see Tree Segment 4, p. 78). The continuations of Sketches E and E1 try to show the interaction of the entering fluids with the stratigraphy. In bedded units one expects unsaturated/saturated flow (matrix flow) as predicted by the composite model (or equivalent), while the welded units (which can support fractures) are more likely to experience fracture flow with leakage to the matrix. The contrasts in hydraulic properties, along with the slight stratigraphic dip and the fault offsets, are expected to contribute to lateral diversion downdip in bedded units and possible local saturation against faults. Whether this diversion occurs as expected could be tested using data on the hydrologic properties of the fault. For example, Sketches F and F1 suggest flow down faults and into fractures in the Topopah Spring unit, with enough lateral conduction to reach the repository.

In both the saturated and unsaturated flow cases (Sketches E1 and E), flow in the channel and in the alluvium is ephemeral. The geometry of the flow is not simply vertical but includes a lateral down-dip component (Mualem and Bear. 1978) and is best represented by a distorted cylinder under and in contact with the flow channel and touching bedrock erratically. Flow is three-dimensional. Further constraints on the persistence of these "flow cylinders" associated with amounts and frequency of precipitation are under investigation by the U.S. Geological Survey (USGS).

\section{Saturated Flow to Bedrock}

Now we will examine each path in Tree Segment 1. The source of water, the surface-flow channel in the wash, produces a saturated flow to bedrock in the alluvium (Sketch E1) followed by fracture flow through bedrock (Sketch F1) to a position where interaction with the repository can occur. Some portion of the water in the flow channel 


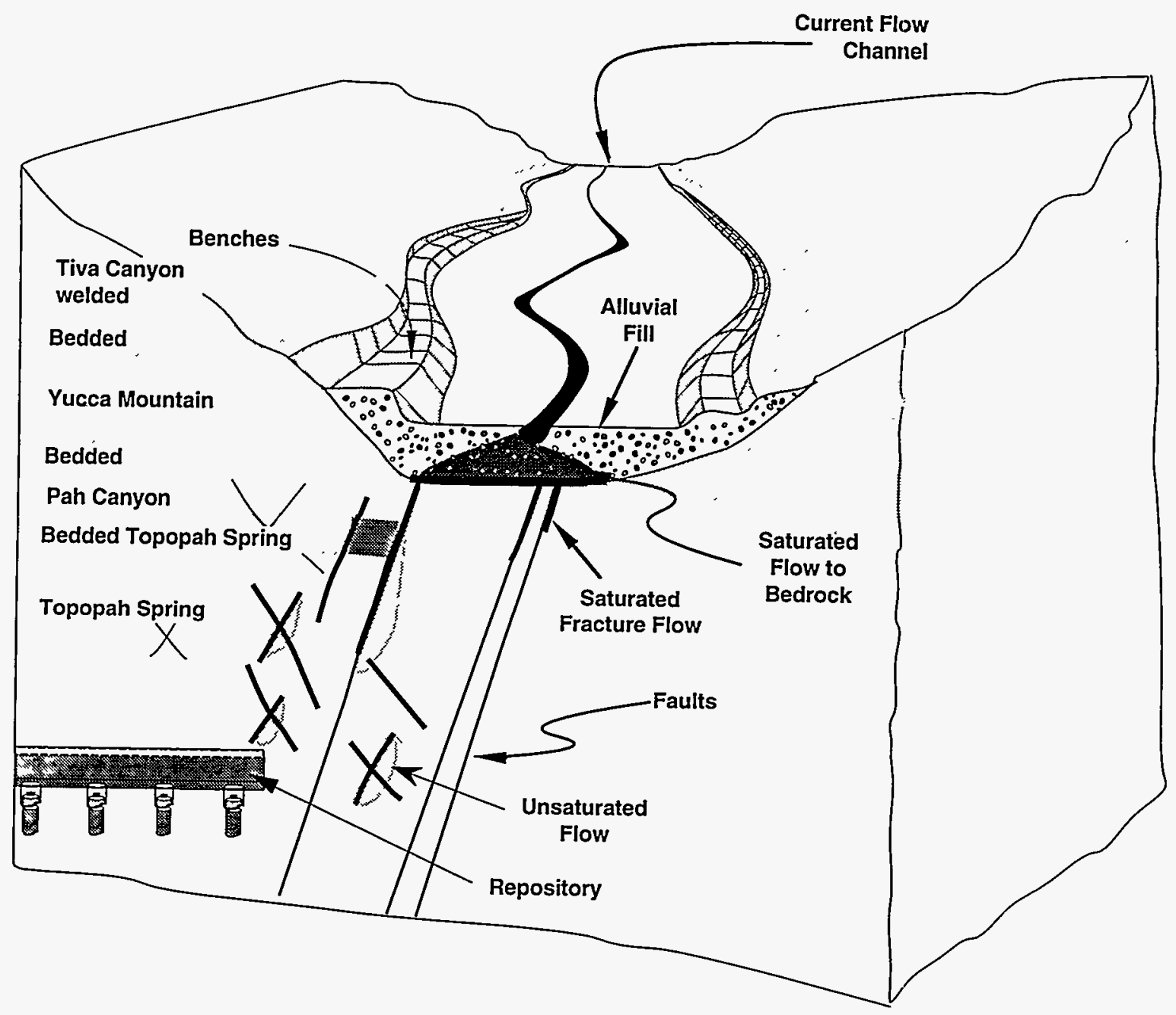

Sketch F1. Large-scale diagrammatic cross section of a wash with faults, showing saturated flow through the alluvium followed by downward and lateral flow in faults and fractures to repository depth. 


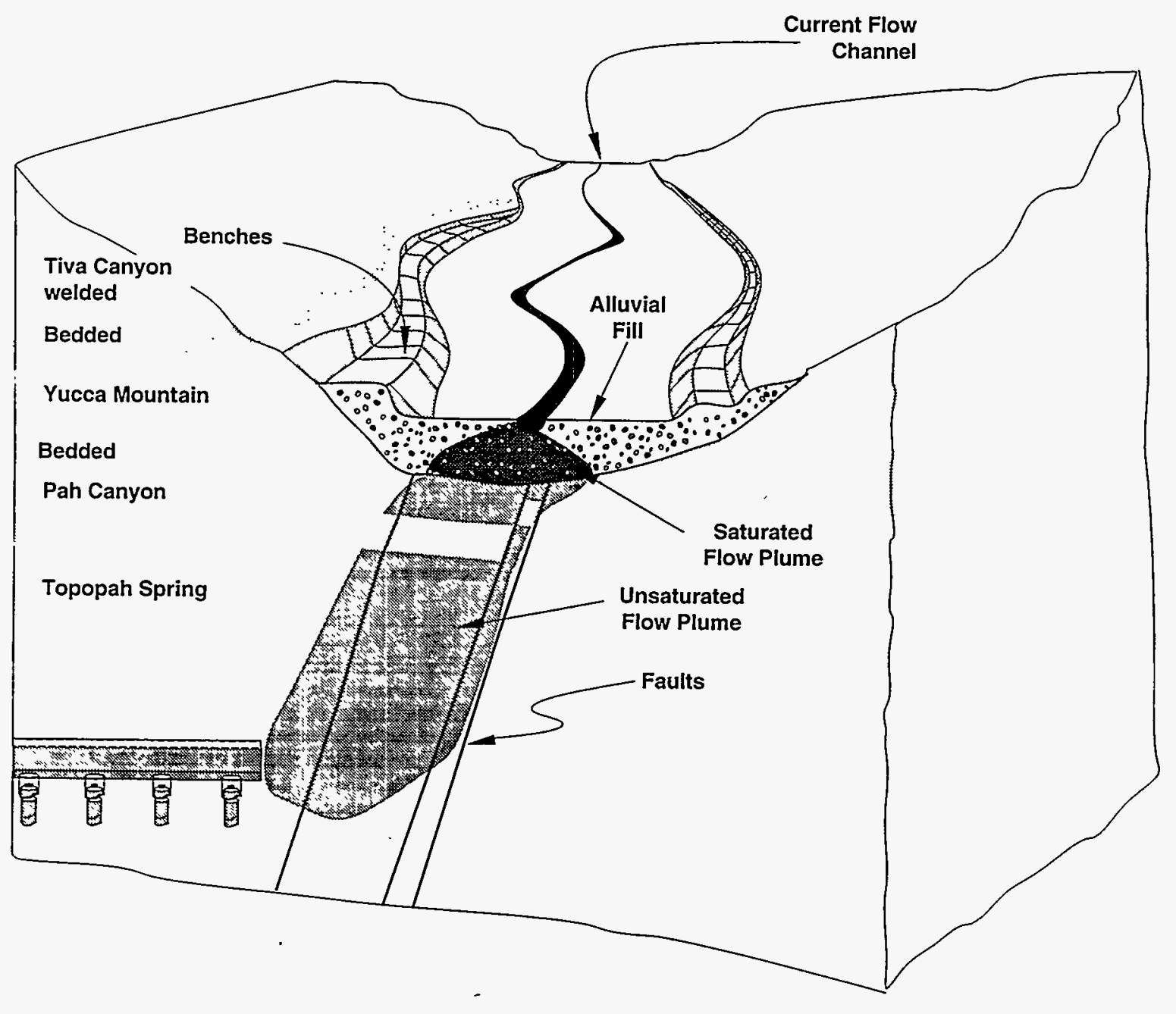

Sketch F2. Saturated flow in alluvium followed by unsaturated flow in bedrock. 


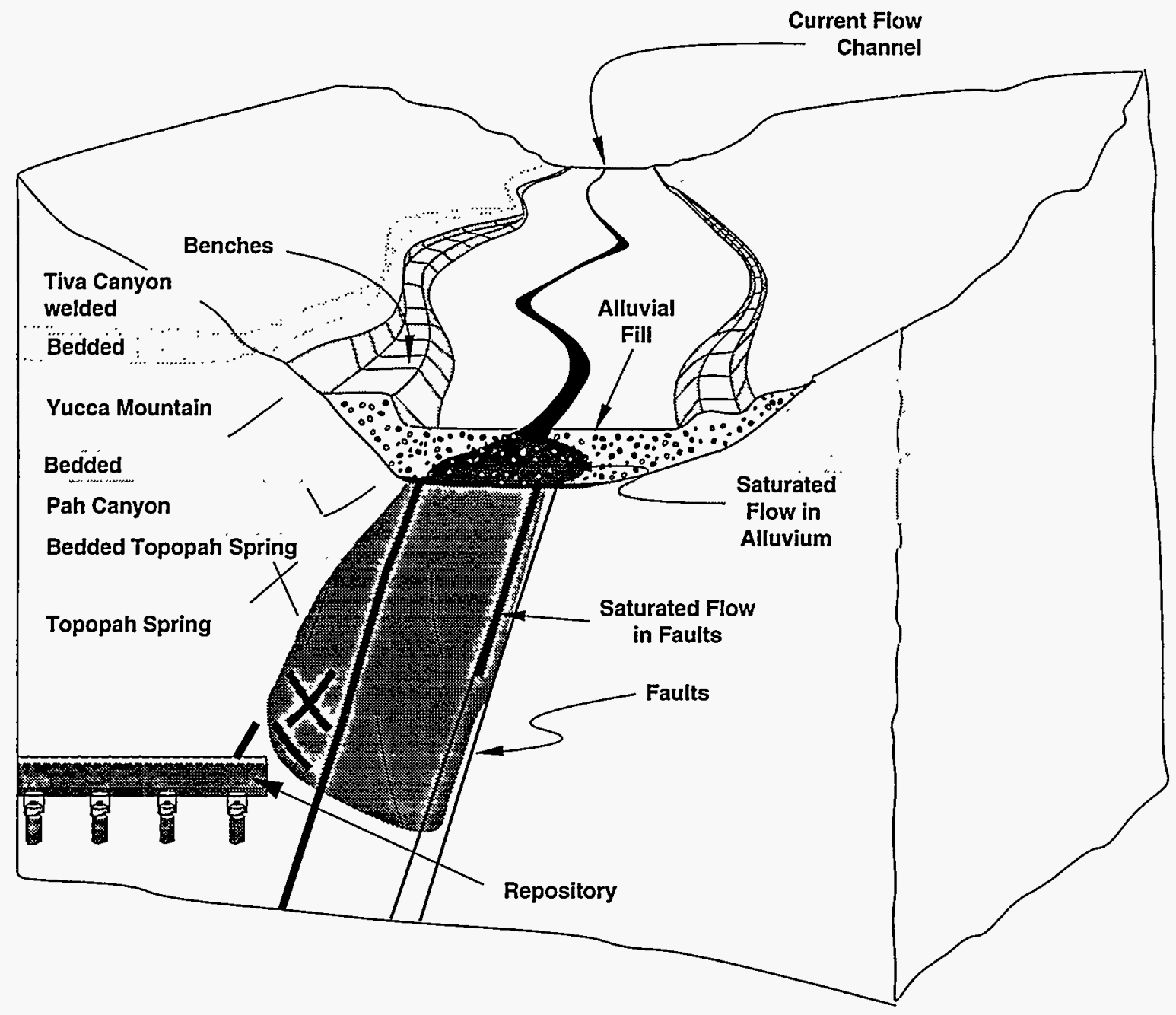

Sketch F3. Saturated flow in faults to repository depth from the alluvium in a wash with faults. 


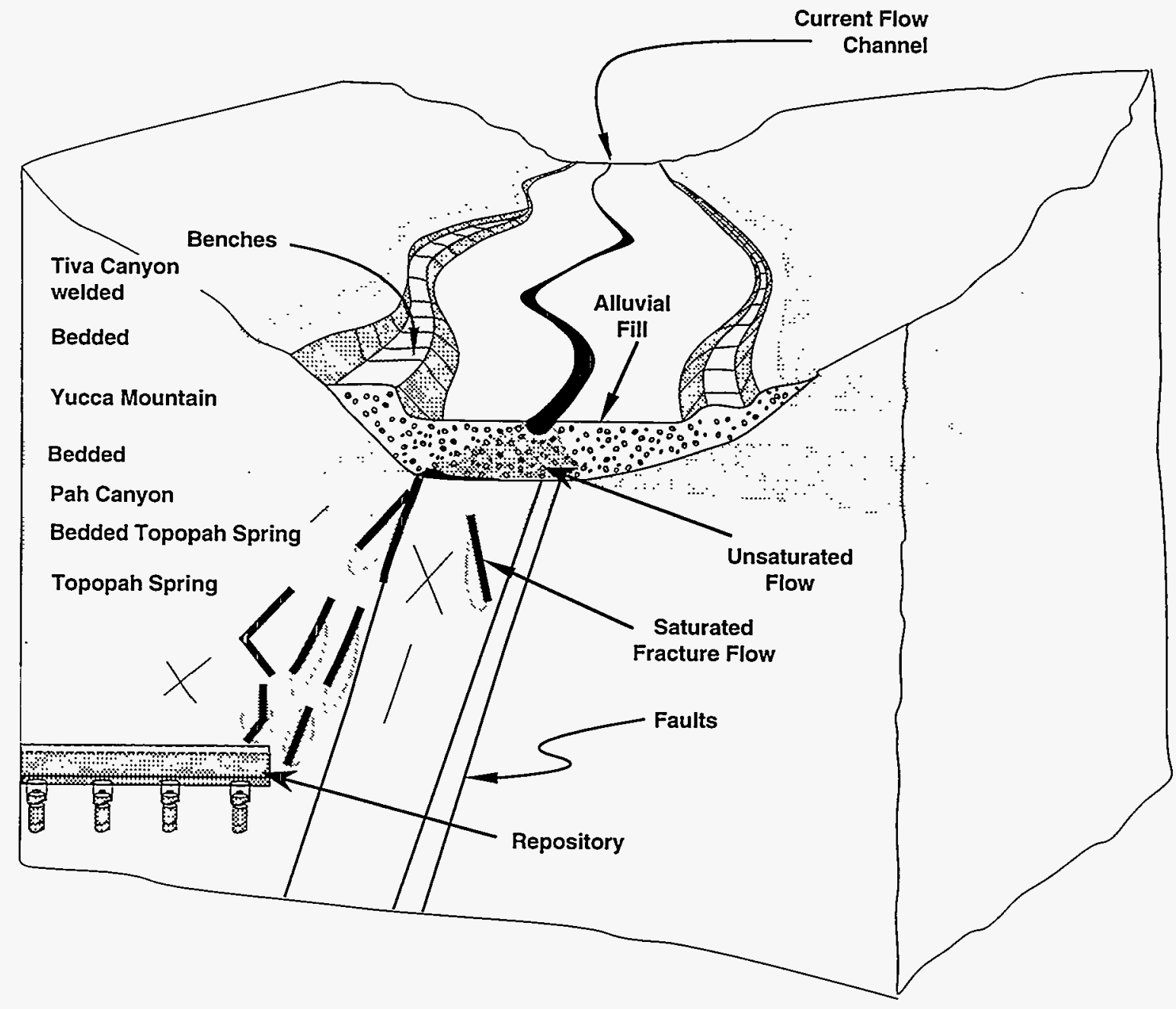

Sketch F. Large-scale diagrammatic cross section of a wash with faults, showing unsaturated flow through the alluvium followed by downward and lateral flow in faults and fractures to repository depth. 
infiltrates the alluvium in the wash. Paths 1.1 through $\triangle 1.6$ describe infiltration at a rate sufficient to saturate the alluvium below the channel down to bedrock (Sketch E1). The formation of such a saturated plume to bedrock requires some minimum frequency and duration of runoff events. Once fluid reaches bedrock, it can reach the repository in a number of ways; Paths $\widehat{1.1}$ through $\widehat{ }$ consider fracture flow through bedrock to the region where fluids begin to interact with the repository (Sketch F1). The extent of the region of interaction depends on the temperature of the repository and the frequency and interconnectedness of the fractures.

\section{Fracture Flow Through Bedrock, Hot Repository}

We define "hot" to mean that the vaporization isotherm is outside the waste container and "cold" to mean that the vaporization isotherm is inside the waste container. A hot repository provides a thermal driving source for twophase flow. We recognize that two-phase systems are somewhat more complicated, but this definition is a convenient choice for this report. By our definition, parts of the repository can be hot and others cold at the same time. The location of the vaporization isotherms will depend on repository layout and waste loading. It is unclear whether 10,000 years will be sufficient for the repository to return to the ambient temperature. Presumably when more data are available to support calculations and thermal output of containers has been chosen, this will be resolvable.

Our definition of a hot repository allows us to identify three different two-phase flow regimes: formation of condensation caps, formation of heat pipes, and venting of vapor from the mountain. A condensation cap is a zone of condensation of vapor generated from-fluid in the rock by the waste-container heat. It may be associated with multi-phase convective circulation on the scale of a single container, a row of containers, or the repository as a whole, depending on the energy and power density of the waste and the time since emplacement. A heat pipe is an engineered device that transfers heat from a heat source to a heat sink by vaporizing a working fluid at the heat source and condensing it at the heat sink. In our case, the heat source is a waste container and the working fluid is the fluid in the rock. The heat sink is colder rock away from the container. Venting is simply the escape of vapor from the mountain. With the natural variation in rock properties, it is conceivable that all three will operate at some time during the hot period either consecutively or simultaneously in different locations. Data and in situ experiments on two-phase flow in fractured porous tuff will allow resolution of which two-phase flow regime dominates.

Path 1.1. The condensation cap in Path $\widehat{1.1}$ is shown in Sketch $\mathrm{H}$, which provides an idealized view of large-scale two-phase movement of fluid around a repository (Buscheck and Nitao, 1993). However, not all calculations that model the rock above the repository as an equivalent porous mediurn show a condensation cap (Pruess et al., 1988). The modeling done by Pruess and others shows a zone of condensation on smaller scale, however, and possibly the inclusion of more detailed properties, e.g., impermeability of the Tiva Canyon welded unit, will produce the cap. In addition to the possibility of cap formation, the volume of fluid available and the rate of return are questions to be resolved.

While two-phase flow is occurring at a rate high enough to counter any influx of liquid to the waste containers, dissolved solids will precipitate wherever evaporation has taken place. Mineral solubility is also a function of 


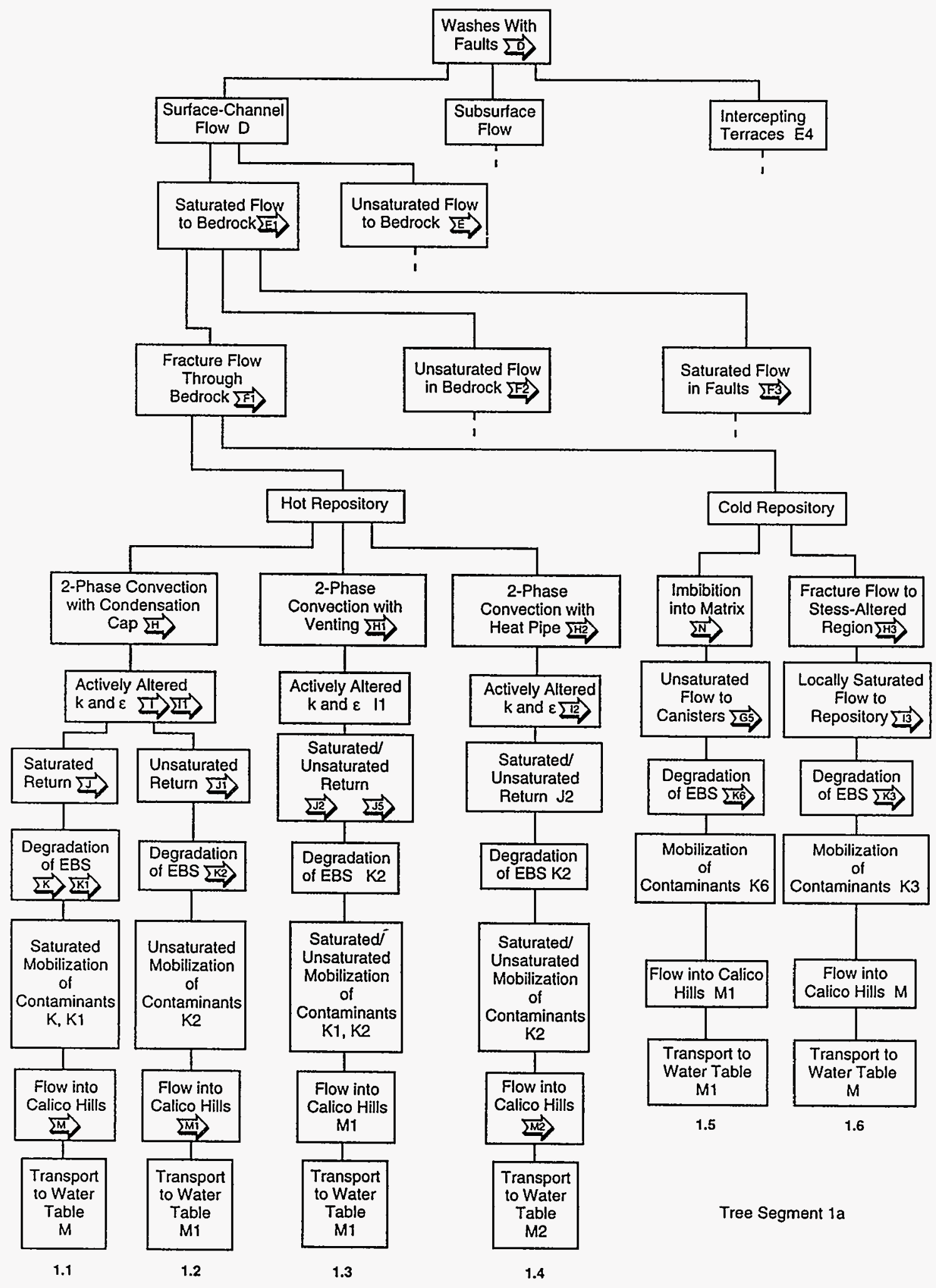

a. Events and their relationships, with cross-references to sketches illustrating each event.

Tree Segment 1. Nominal Flow, Runoff-Producing Events, Runoff to Washes, Washes With Faults, SurfaceChannel Flow, Saturated Flow to Bedrock, Fracture Flow Through Bedrock. 


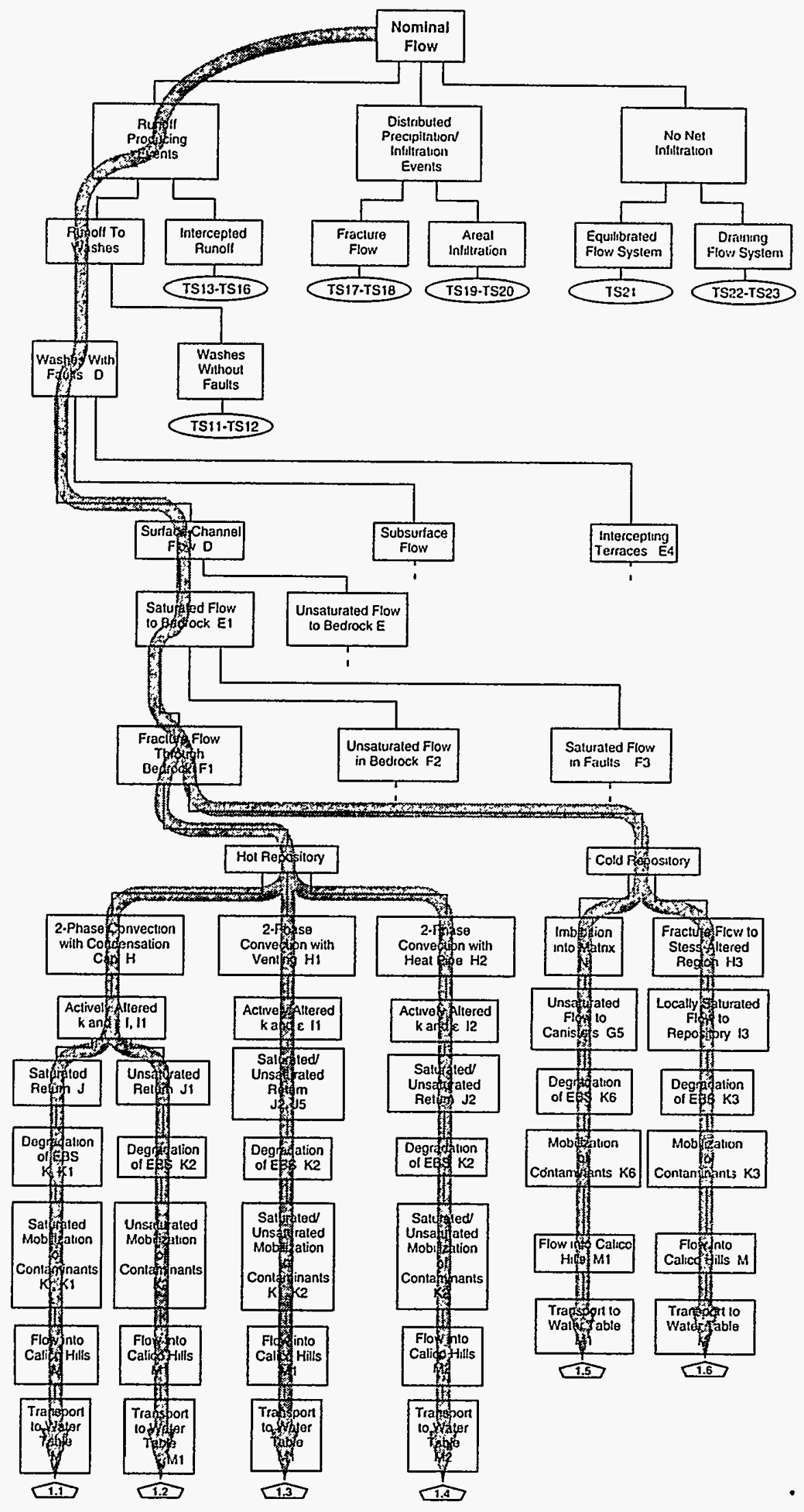

b. Scenario pathways, showing the relationship of the tree segment to the overall event tree for nominal flow. 

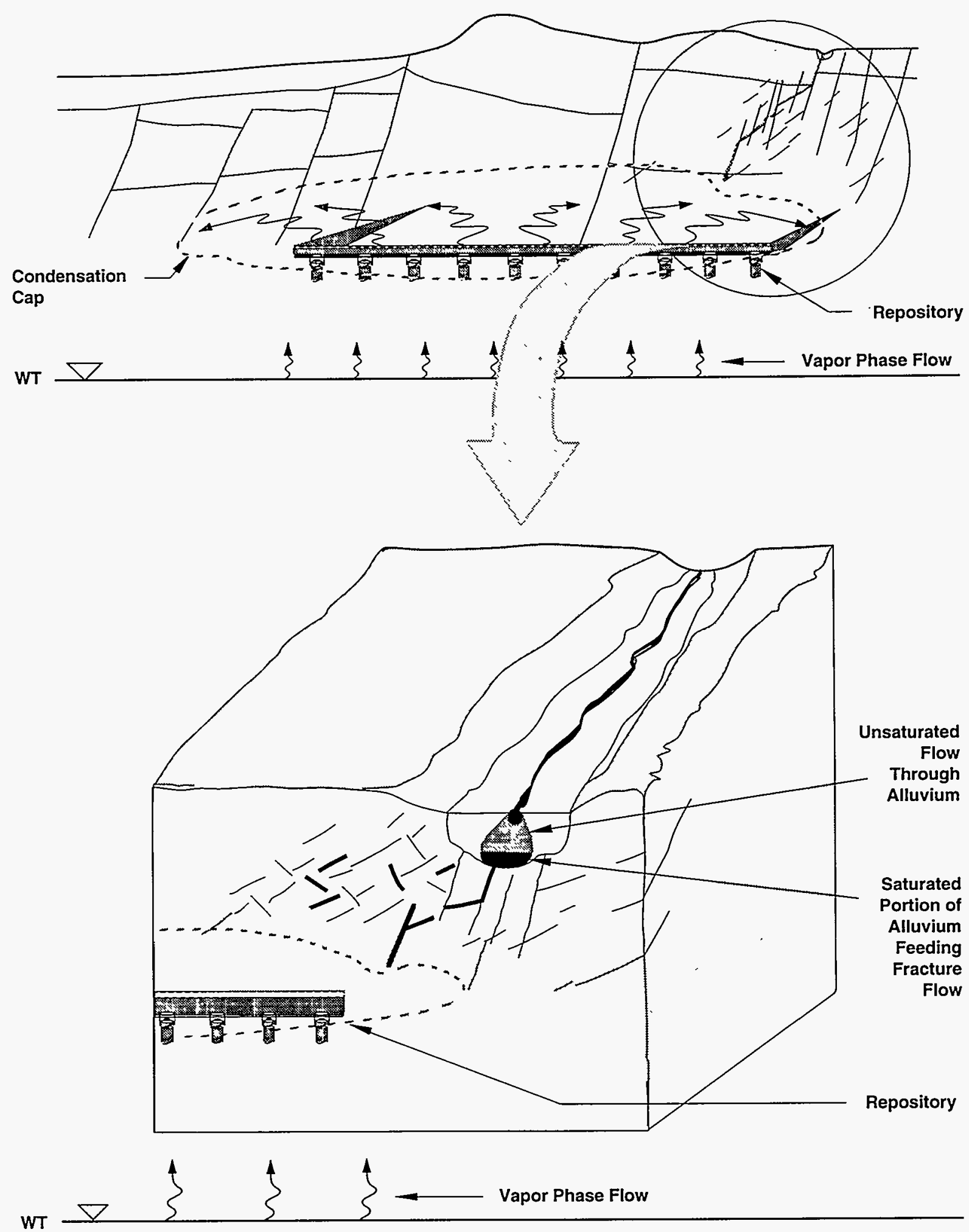

Sketch H. Idealized view of the interaction between fracture flow from the wash and the condensation cap formed by heat-driven two-phase fluid flow around a repository. 
temperature. Presumably these precipitates will alter connected porosity in the matrix and fractures and therefore affect permeability and porosity in the immediate vicinity of waste container and drift. Whether these alterations are detrimental to repository performance is still an open issue. Sketch I (p. 15) shows a general cross section of waste container, drift and backfill, and induced and previously existing fractures. The extent and mineralogy of any pore and fracture fillings must be determined to guide inclusion of the chemistry in calculational models. These two factors will influence the hydrologic and geochemical properties of the units. Water vapor may condense outside the isotherm and accumulate as a liquid to return at a later time (Sketch I1). Little or no aqueous transport is possible until liquid returns from the condensation cap and containers fail.

After the rock and repository have cooled sufficiently to allow liquids to persist at waste containers, the condensation cap may act as a source of returning fluids. Sketch J indicates such return of fluid down induced and previously existing fracture sets. Previously existing fractures, and any filling of pores and fractures by mineral deposition, constrain the volume and rate of fluid delivery to cooling waste containers.

Path $\widehat{1.1}$ next considers how the waste container and associated emplacement devices (if any) - the EBShave fared. Even though "Degradation of the EBS" has been placed after the saturated return in the tree, the flow of hot water vapor past the waste container in the annulus around the cans may have already compromised the system much earlier in the repository history, even as early as the time of emplacement. Although container degradation may have been suspended, at least externally, during dryout, it resumes when liquids can reach the containers. (It is possible for container corrosion to continue by vapor action during the dryout period. The mass removal rate may be different for corrosion in the presence of a liquid as opposed to a vapor, but not the chemical potential at the same conditions.) We have indicated the degradation of the EBS in such a way as to include saturated flow to the waste container.

Degradation and mobilization are indicated in Sketches $\mathrm{K}$ and $\mathrm{K} 1$. Sketch $\mathrm{K}$ illustrates the case of such voluminous reflux that the bottom of the waste container is submerged; Sketch K1 illustrates drip or flow of liquid onto the waste container. Initial flow and transport away from the waste containers are by both fracture and matrix - saturated and unsaturated flow. Entrance and exit of fluid to and from the waste container are presumed to occur through openings caused by either corrosion damage from water vapor or corrosion by the liquids making contact during reflux. Mobilization of contaminants and their leakage from the emplacement hole are shown in Sketches $\mathrm{K}$ and $\mathrm{K} 1$. Flow down connecting fractures and flow in fingers in an unsaturated mode are both indicated (Glass and Tidwell, 1991; Nicholl et al., 1993). Contaminants are presumed to accompany this flow, which acts as the source for transport in the Calico Hills units and to the water table.

Transport to the water table is shown on a large scale in Sketch M. Continuity and connectivity of fractures in the units are yet unknown. We have constructed a scenario, Path $\widehat{1.1}$, described by Sketches C, D, E1, F1, H, I, Il, J, K, K1, and M. In it, runoff to washes with faults flows down fractures in bedrock to interact with a hot repository under circumstances allowing production of a condensation cap and eventual saturated reflux. 


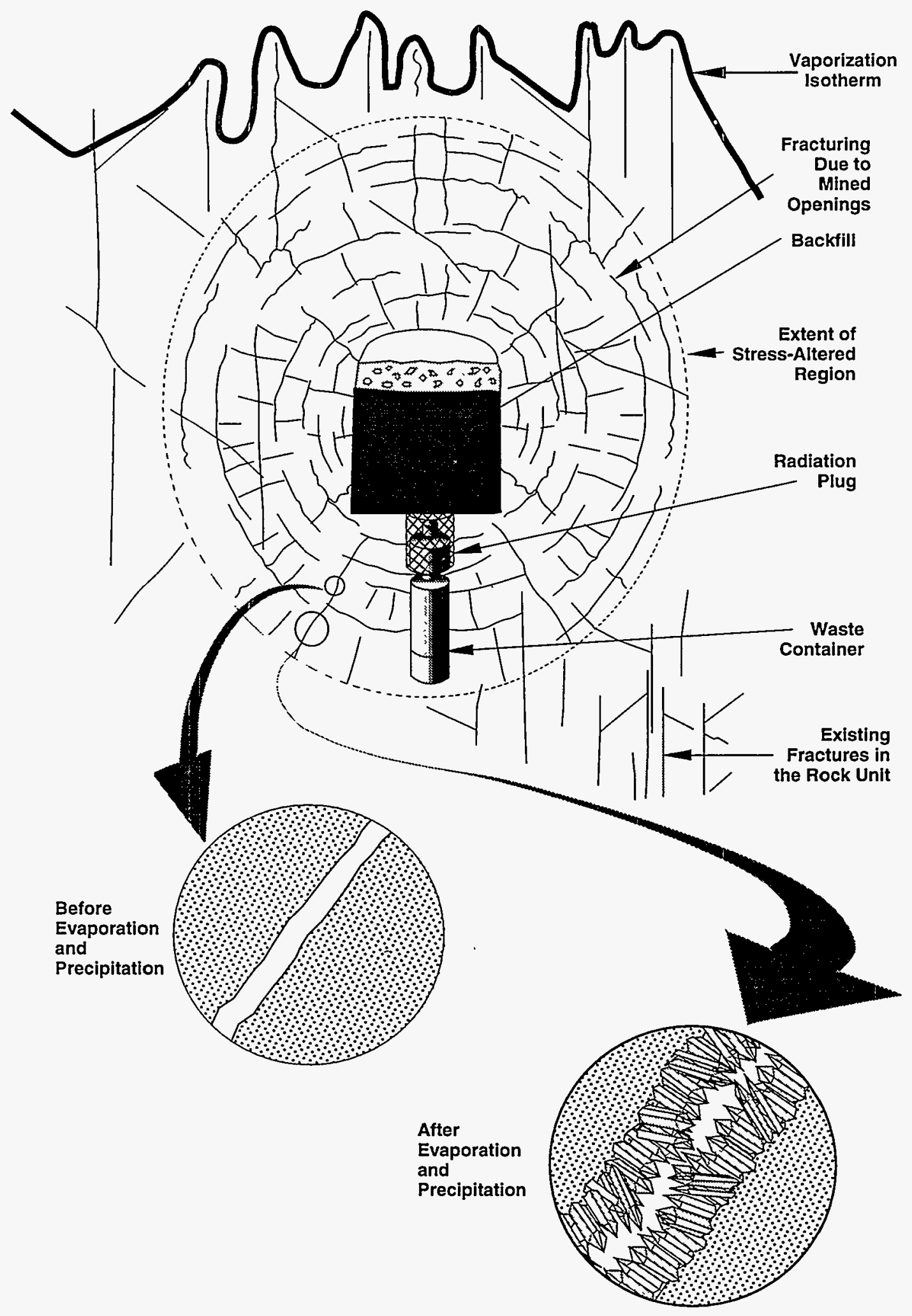

Sketch I1. Diagrammatic cross section of waste and drift showing the vaporization isotherm and dryout zone. The enlargements show the very-small-scale process of alteration of permeability and porosity. 

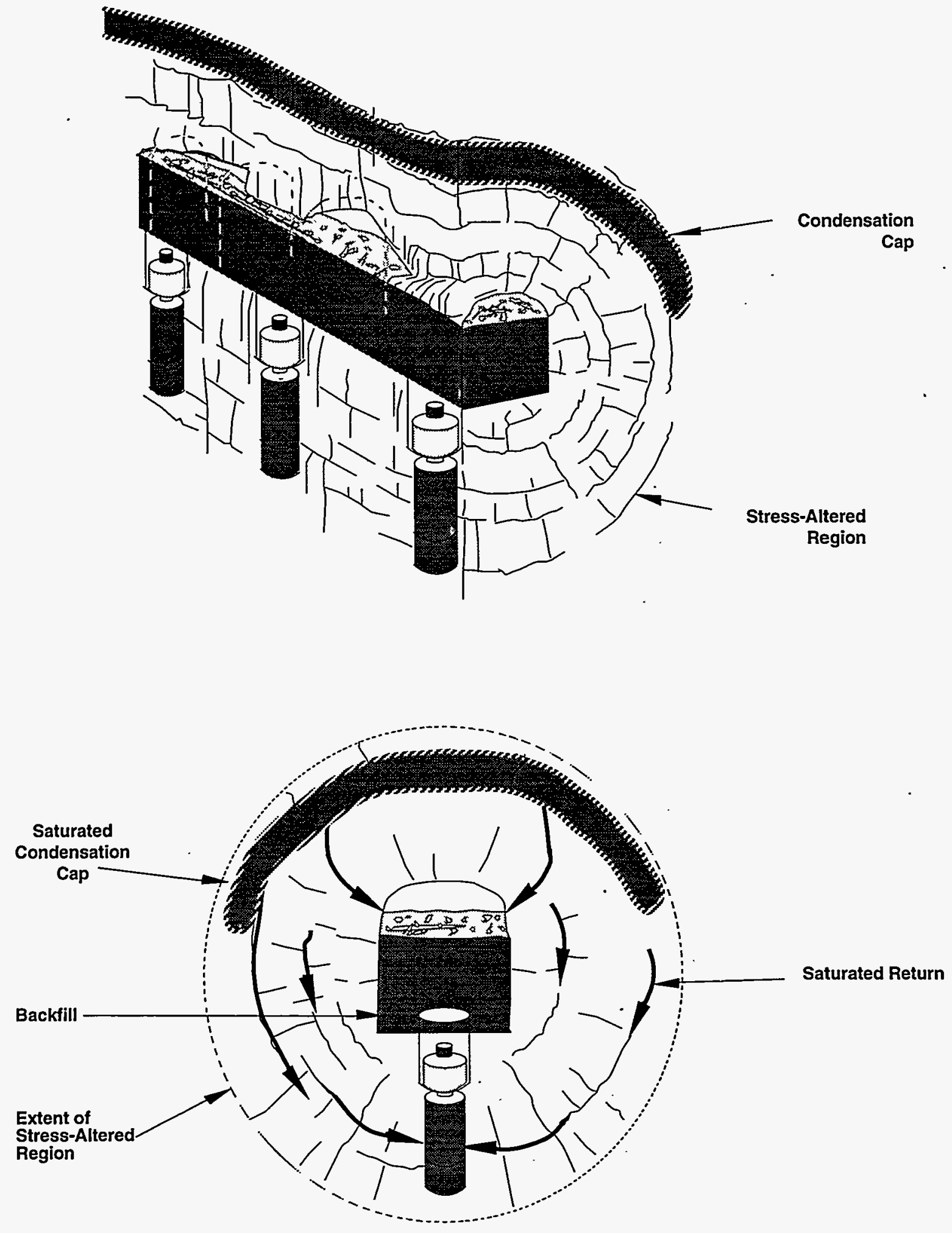

Sketch J. Saturated return of liquids from the condensation cap to the repository. The major portion of the return flow travels through the fractures. Fractures are three-dimensional and may intersect outside the plane of the sketch. 


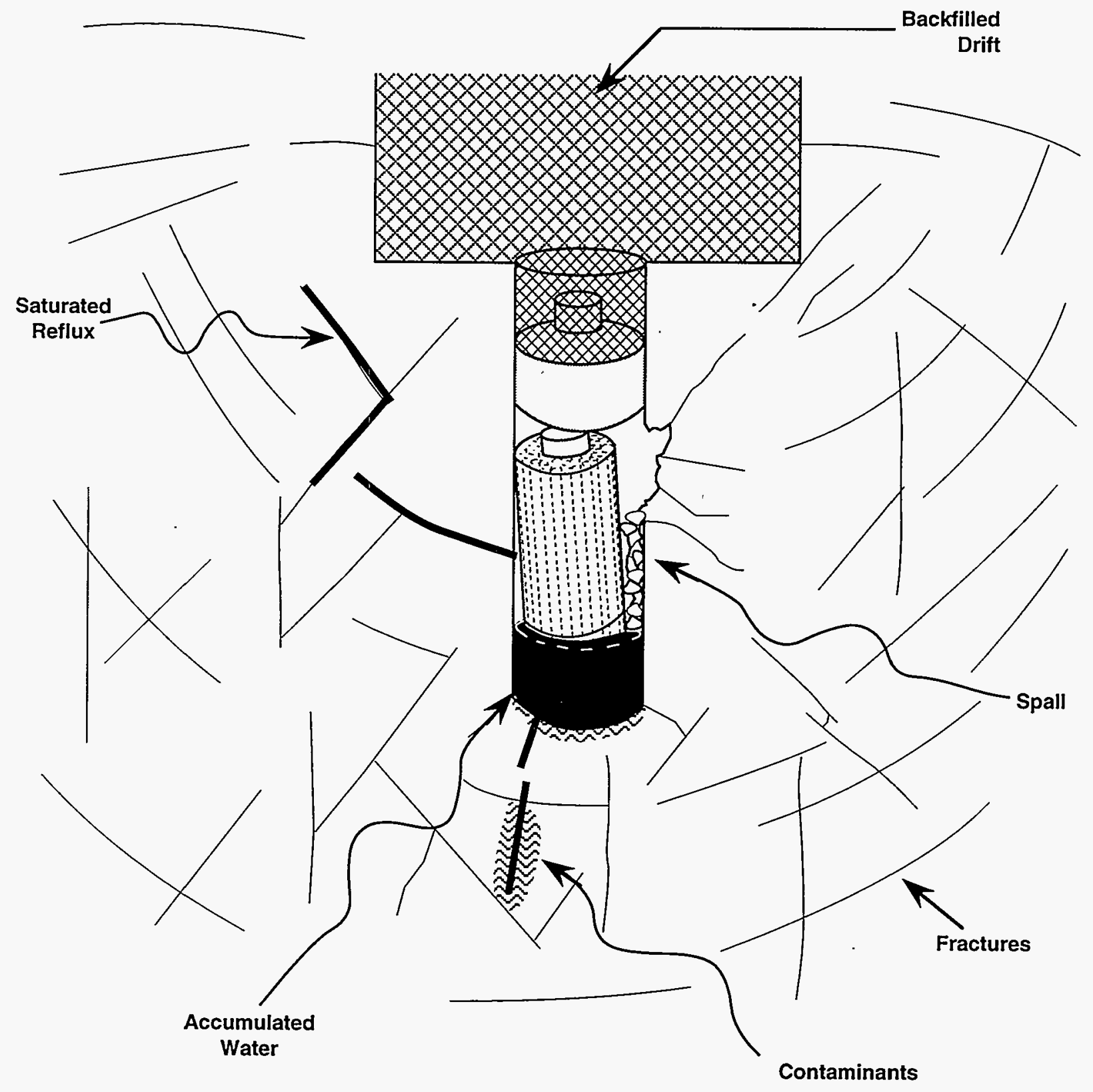

Sketch K. Degradation of the EBS and mobilization of contaminants in the presence of voluminous reflux that partially submerges the waste container. 


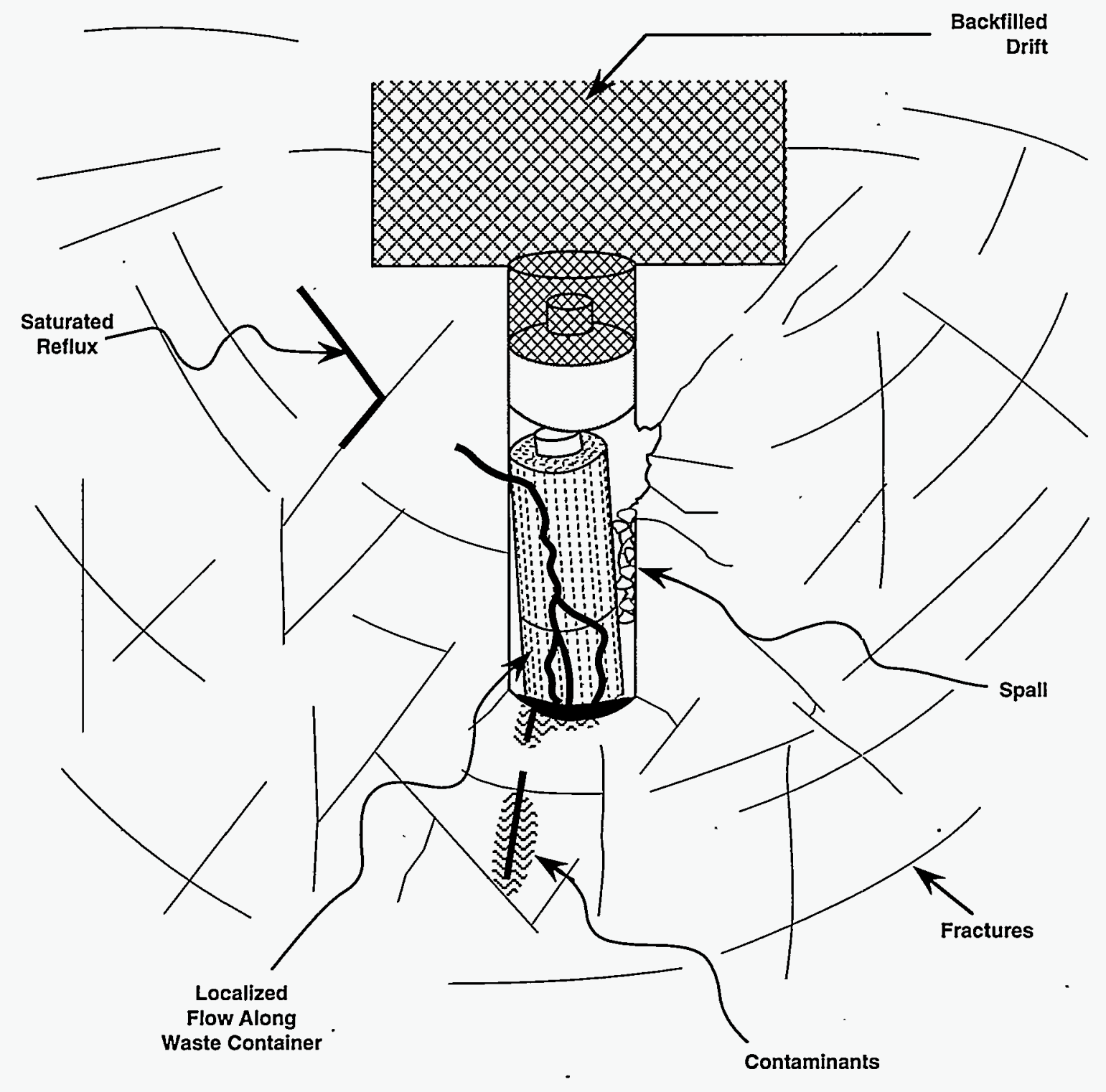

Sketch K1. Degradation of the EBS and mobilization of contaminants in the presence of meager reflux that drips onto the waste container. 


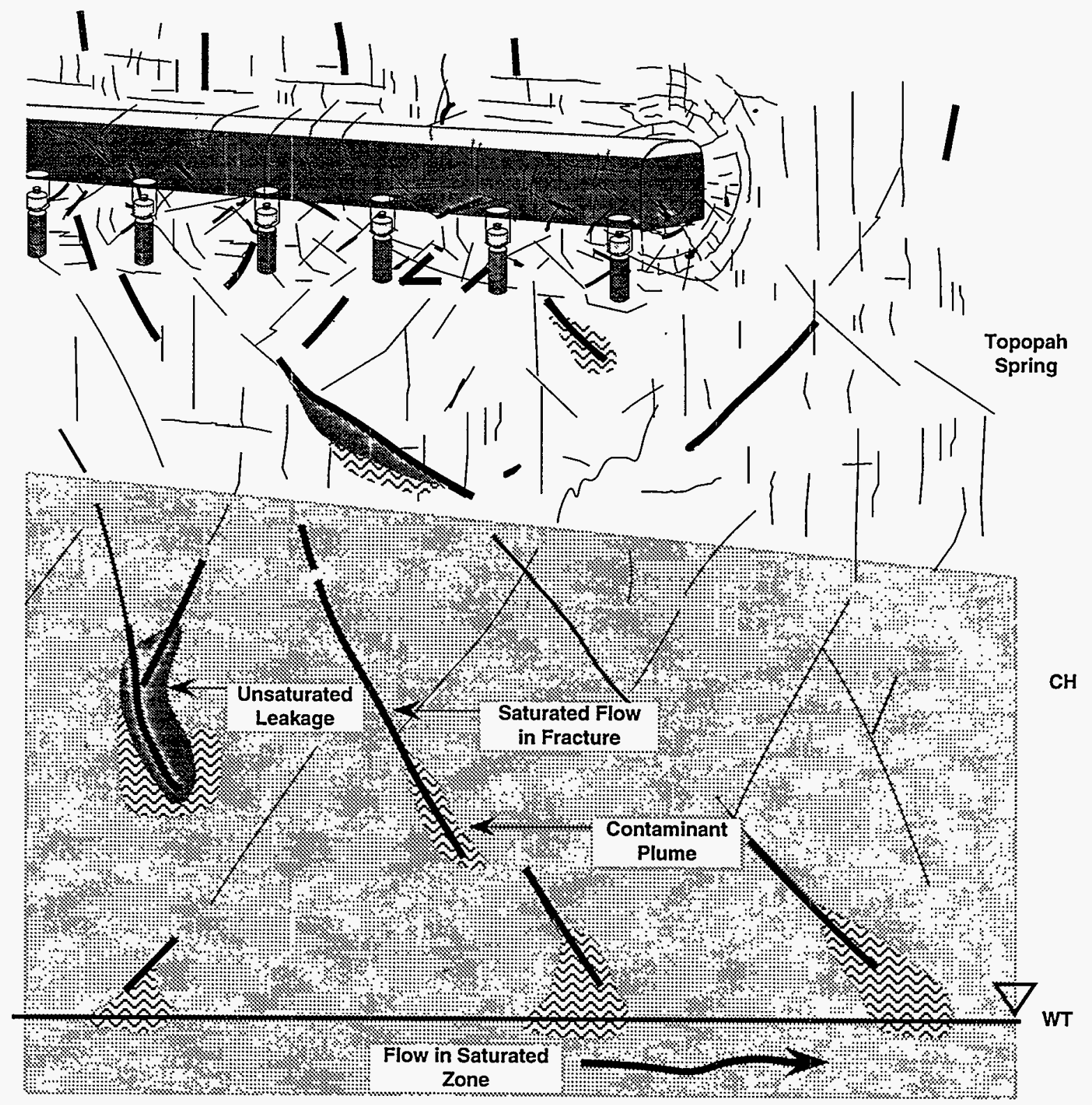

Sketch M. Transport of contaminants from the repository to the water table, showing saturated fracture flow to the waste container and transport in saturated fractures to the water table. $\mathrm{CH}=$ Calico Hills units; WT $=$ water table. 
Path 1.2. The discussion of Path $\widehat{1.1}$ presumed a saturated reflux from the condensation cap to the repository. An unsaturated return is also possible and is in the tree as a separate path (Path 1.2 ) parallel to the "Saturated Return." In either case the fractures in the stress-altered region confine and redirect the flow. The two cases are distinguished because the time scales are likely to differ considerably for each type of flow, leading to substantially different modeling requirements.

Following Path 1.2 for the hot repository leads to "Unsaturated Return" (Sketch J1). This figure mimics Sketch J, but the return flow from the condensation cap must be outside most fractures-except for locally saturated seeps and weeps. Backfill in the drifts could participate in conducting this flow if its porosity is sufficiently small. The next steps, "Degradation of EBS" and "Unsaturated Mobilization of Contaminants," are illustrated in Sketch K2. Contact of the unsaturated flow field with a waste container requires some sort of bridging to provide a continuous path. Contact is indicated in Sketch $\mathrm{K} 2$ as spall. The contaminants are mobilized to enter the unsaturated flow field below the waste container. Three different forms of transport are indicated in the sketch: a contaminated, unsaturated flow plume; a gravity-driven contaminant finger (Glass, 1989; Glass and Tidwell, 1991; Nicholl et al., 1993); and a contaminated zone of local saturation (i.e., perched water flow). Each of these may have different speeds of movement and volumetric flow rates. Movement away from the waste containers and transport to and in the Calico Hills units (Sketch M1) are similar to Path 1.1 , except for unsaturated transport away from the containers. A second scenario, Path 1.2 , is thus identified by Sketches C, D, E1, F1, H, I, I1, J1, K2, and M1 for runoff to washes with faults, saturated flow to bedrock, and fracture flow through bedrock to interact with a hot repository under circumstances allowing production of a condensation cap and eventual unsaturated reflux.

Path $\widehat{1.3}$. Two-phase convection may lead to venting (Path $\widehat{1.3}$ ); no condensation cap forms because large amounts of vapor escape from the mountain. Any reflux that occurs is a normal return from the unsaturated/saturated flow system in the mountain around the repository. The volume of the dried region will depend on the thermal load and on whether the water table participates as a significant source.

During venting, the fracture system that would ordinarily conduct fluids downward could act as vents for water vapor. Fractures may occasionally be blocked by liquid flow because the source-fluids in a wash with faults-is episodic. Sketch H1 shows how fractures could provide connections from the bottom of the wash to the repository. The sketch also shows a dotted line approximating a condensation cap; the system is not homogeneous, and it is possible for condensation to occur in some regions over the repository while there is venting elsewhere. Vapor produced by repository heat leaves precipitates behind in the matrix pores and in the fractures. Movement of hot fluids increases solubility of some minerals in the fractures. The net result is alteration of permeability and porosity in matrix and fractures. This circumstance is sketched in Sketch II for the region near a drift being fed from a wash with faults. The vaporization isotherm follows fractures somewhat because hot vapor flows into the fractures. However, alterations in permeability and porosity can extend beyond the vaporization isotherm because of temperature-induced changes in mineral solubility. Return flow to the repository, which can occur only when heating has diminished, is by unsaturated flow from cooler rock around the repository, by fracture flow down the 


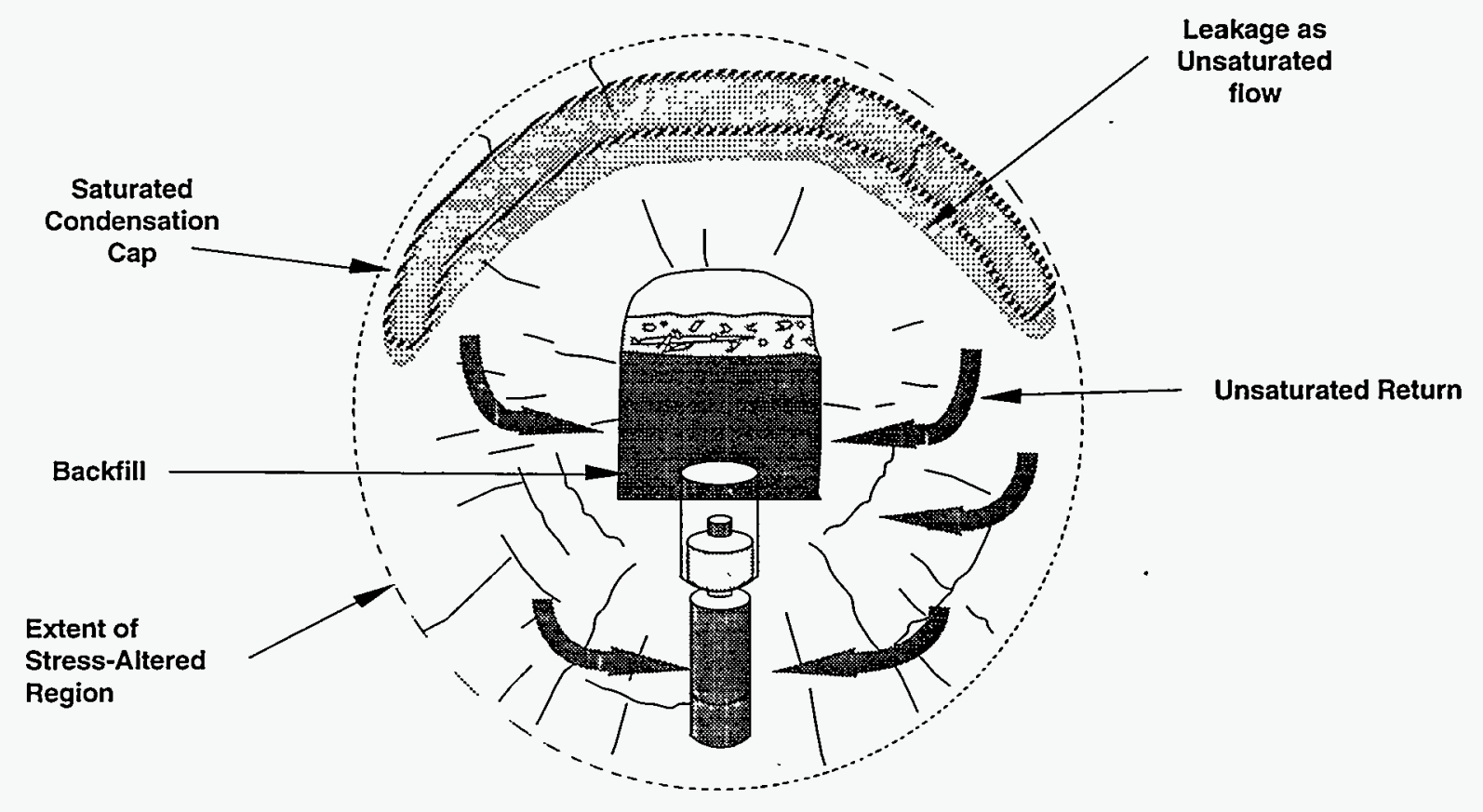

Sketch J1. Unsaturated return of liquids from the condensation cap to the repository. The major portion of the return flow travels through the matrix. 


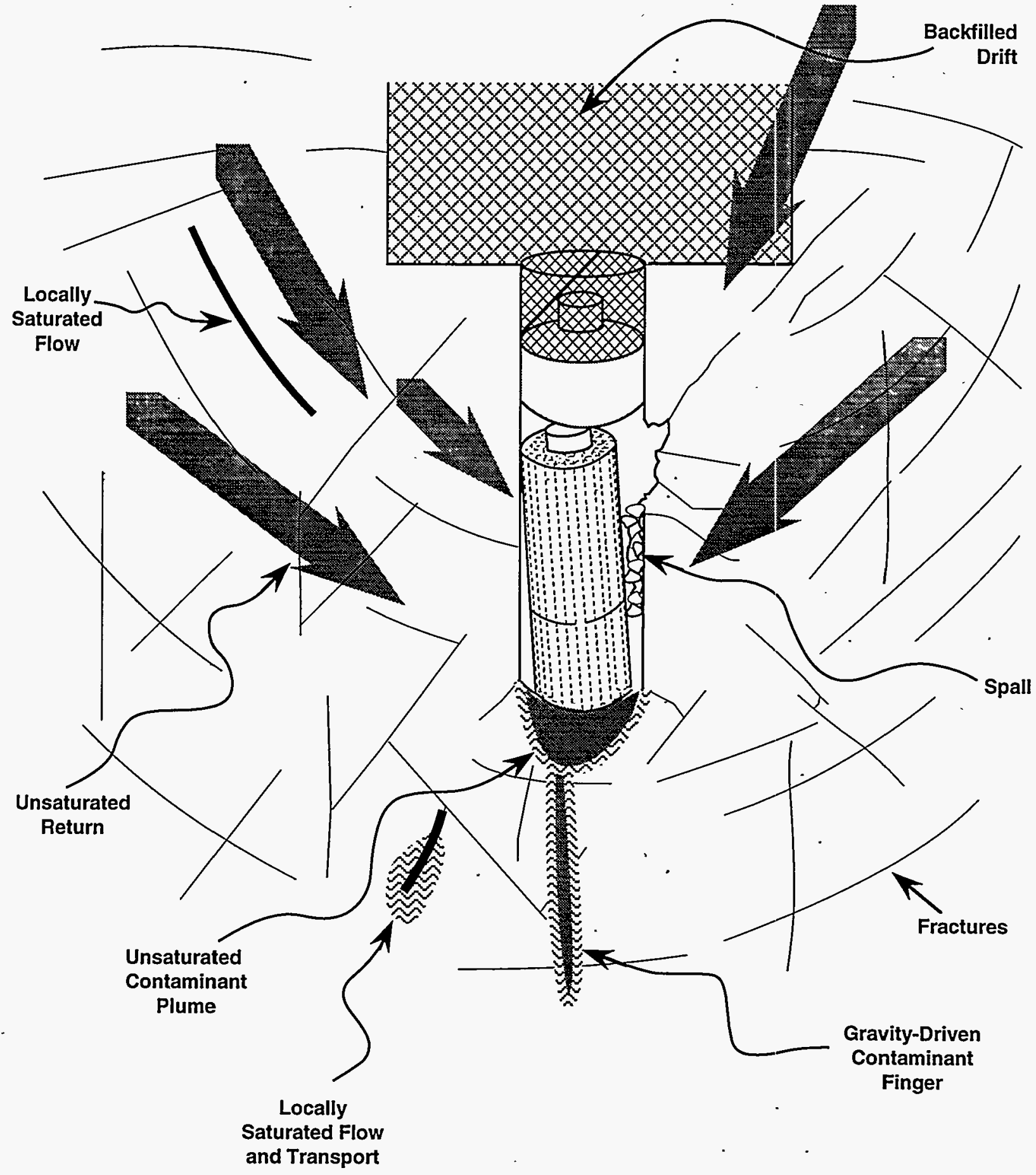

Sketch K2. Degradation of the EBS and mobilization of contaminants in the presence of unsaturated flow. The sides of the emplacement hole must touch the container in some way for liquids to reach the container. * For example, rock spalling from the side of the hole may bridge the gap, or the container may lean against the rock. 


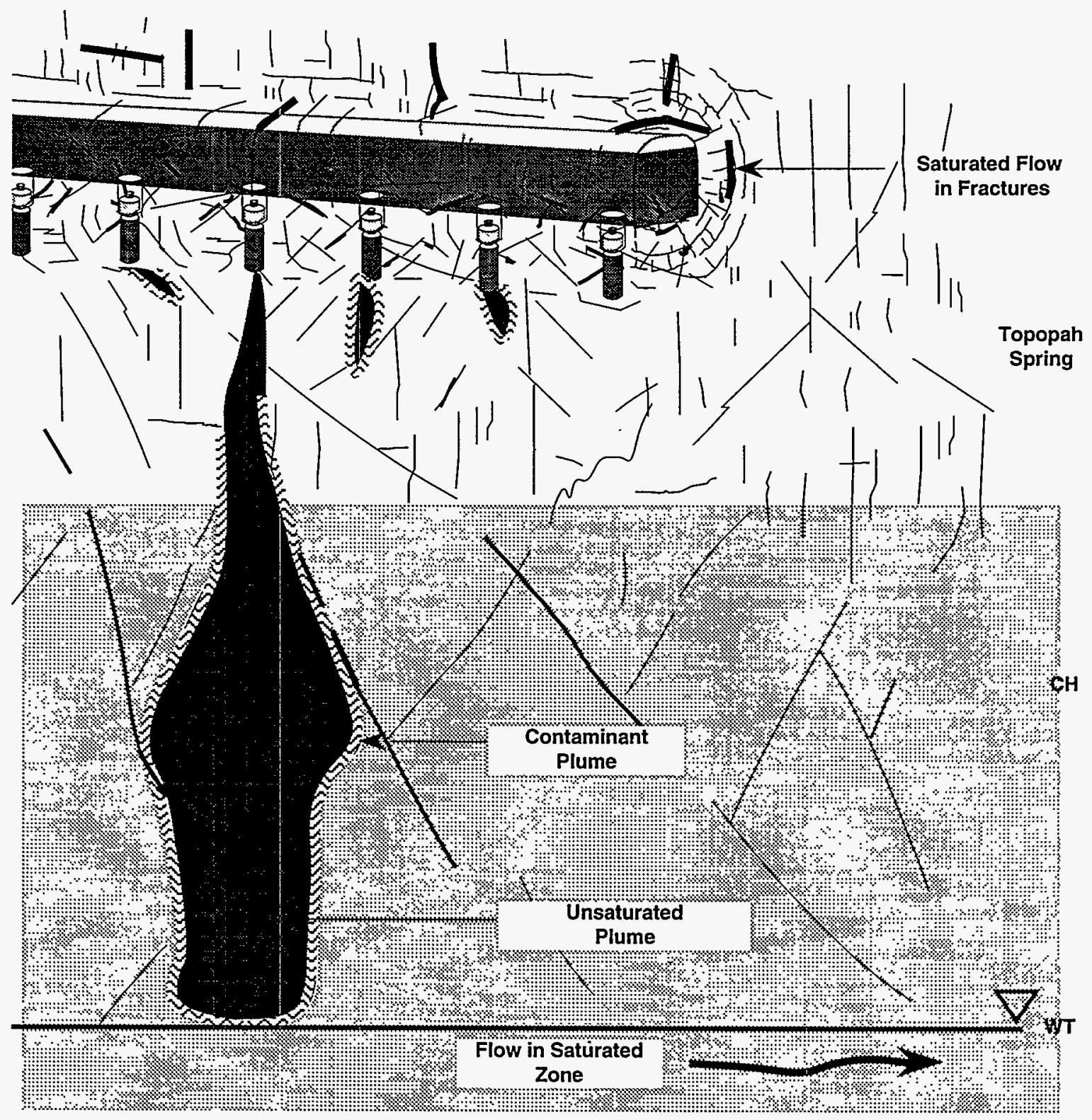

Sketch M1. Transport of contaminants from the repository to the water table, showing unsaturated flow to the waste container and unsaturated transport to the water table. $\mathrm{CH}=$ Calico Hills units; WT $=$ water table. 


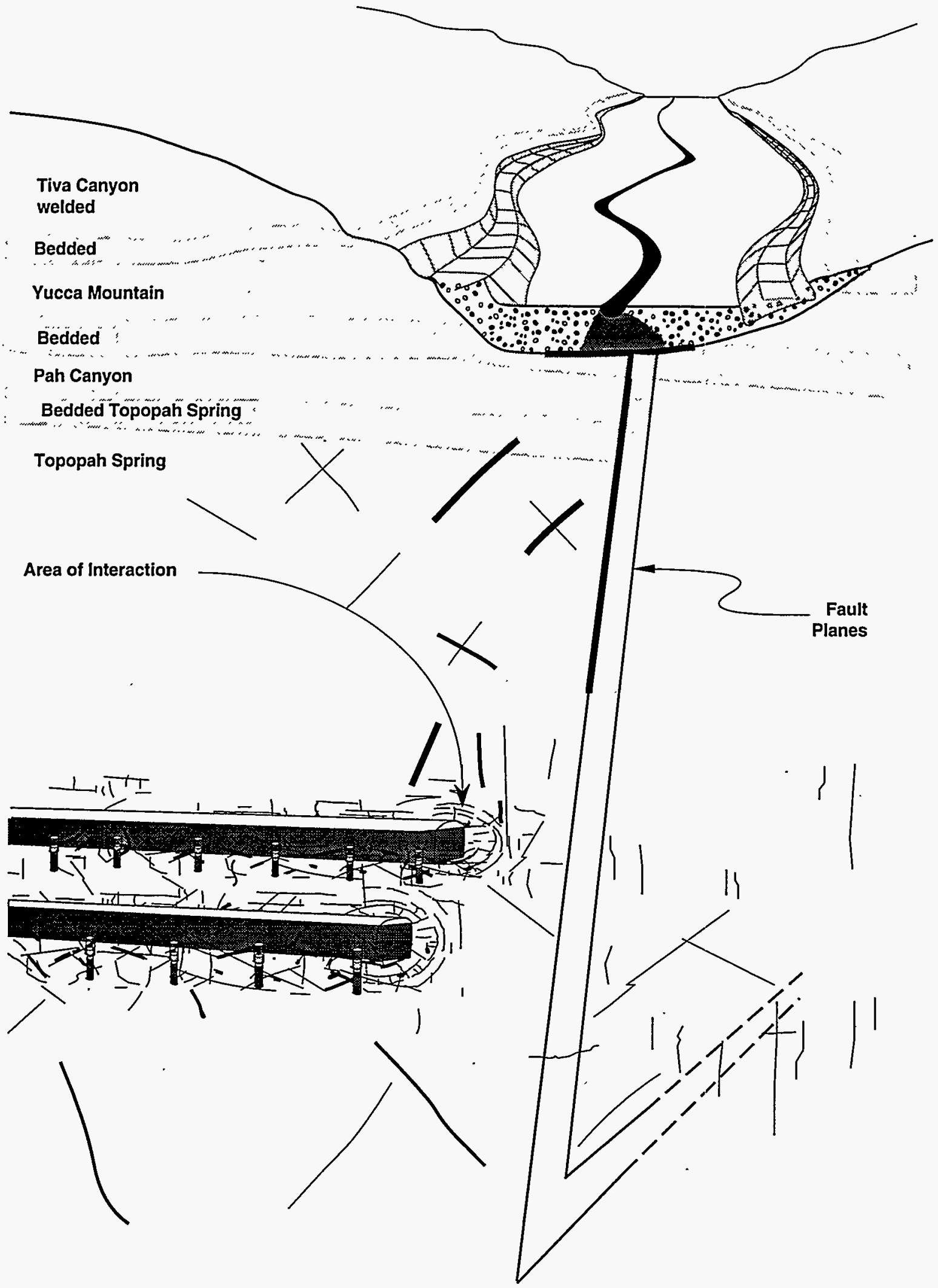

Sketch H1. Fracture flow from a wash with faults. This figure serves as a stand-in for two-phase convection with venting. 
fracture sets connecting with the wash, and by vapor flow back to the repository from surrounding unsaturated rock and the water table. Sketches J2 and J5 are intended to show this return recharge, J2 in large scale extending to the wash and $\mathrm{J} 5$ in small scale around a drift. The return of fluids is accompanied by degradation of the EBS. Actually, the EBS may have previously experienced corrosion due to hot water vapor streaming past, and there may have been thermally or mechanically induced spall. Return fluids exacerbate the degradation. The arrival of fluids and their possible contact with the waste container are shown in Sketches $\mathrm{K} 1$ and $\mathrm{K} 2$ as a stand-in for degradation, an extremely small-scale process that does not lend itself to pictorial representation. The mobilization process is described as unsaturated/saturated to allow for the presence of occasional seeps and weeps near waste containers that are fed by the unsaturated flow system. The degradation of the EBS, a function of temperature, water volume and mode of delivery, and of the waste form (fuel rods, glass), is a matter of current study; its true representation is not yet available. Mobilization of contaminants is depicted in Sketches $\mathrm{K} 1$ and $\mathrm{K} 2$, where the type of mobilization (solute, colloid, etc.) is decided by calculation based on the volumetric flow rate for return and the rock properties after drying. Flow into the Calico Hills units and down to the water table is shown schematically in Sketch M1. The scenario constructed for Path $\widehat{1.3}$ is described in Sketches C, D, E1, F1, H1, I1, J2, J5, K1, K2, and M1.

Path $\widehat{1.4}$. The final branch on the "Hot Repository" limb, Path $\widehat{1.4}$, considers the development of sustained heat pipes. The "heat pipe" in the repository is a natural analog to an engineered heat transfer device in which the working fluid functions in both liquid and vapor phases (Winter and Barsch, 1971). The engineered version of this device efficiently transfers energy by vaporizing a working fluid at one end of a closed cylinder and condensing it at the other, recovering the latent heat of vaporization on condensation. The device has a void space for rapid transfer of vapor and a wick for return of the condensate by capillary forces back to the hot end. In the Topopah Spring units fractures could substitute for the void space, matrix pores for the wick, and water for the working fluid (Sketch H2). The heat source is distributed in the rock around the waste container rather than localized, and the heat sink is likewise distributed. Pruess and coworkers have expended considerable effort to try to understand the details of multiphase behavior in the vicinity of the waste container (Tsang and Pruess, 1987; Pruess and Tsang, 1991). Their analyses have provided guidance for these discussions and for the development of the tree. When underground experiments are available to constrain calculations, we presume their modeling will provide more complete insights into actual long-term behavior of multiphase flow around the repository. The dialogue between experiments and modeling will illuminate the real behavior of this system.

Vaporization and dryout resulting from this heat pipe behavior are expected to alter permeabilities and porosities in the active region by mineral precipitation and alteration. Sketch I2 shows the backfilled drift and the stress-altered region explicitly. Vapor movement is illustrated along the waste container and emplacement hole in this sketch to emphasize the problem of steam corrosion and degradation of the EBS. This effect is not certain; it is at least conceivable that deposition of dissolved minerals on the waste container occurs at some time in the thermal period. If this process is important, it should be addressed experimentally. 


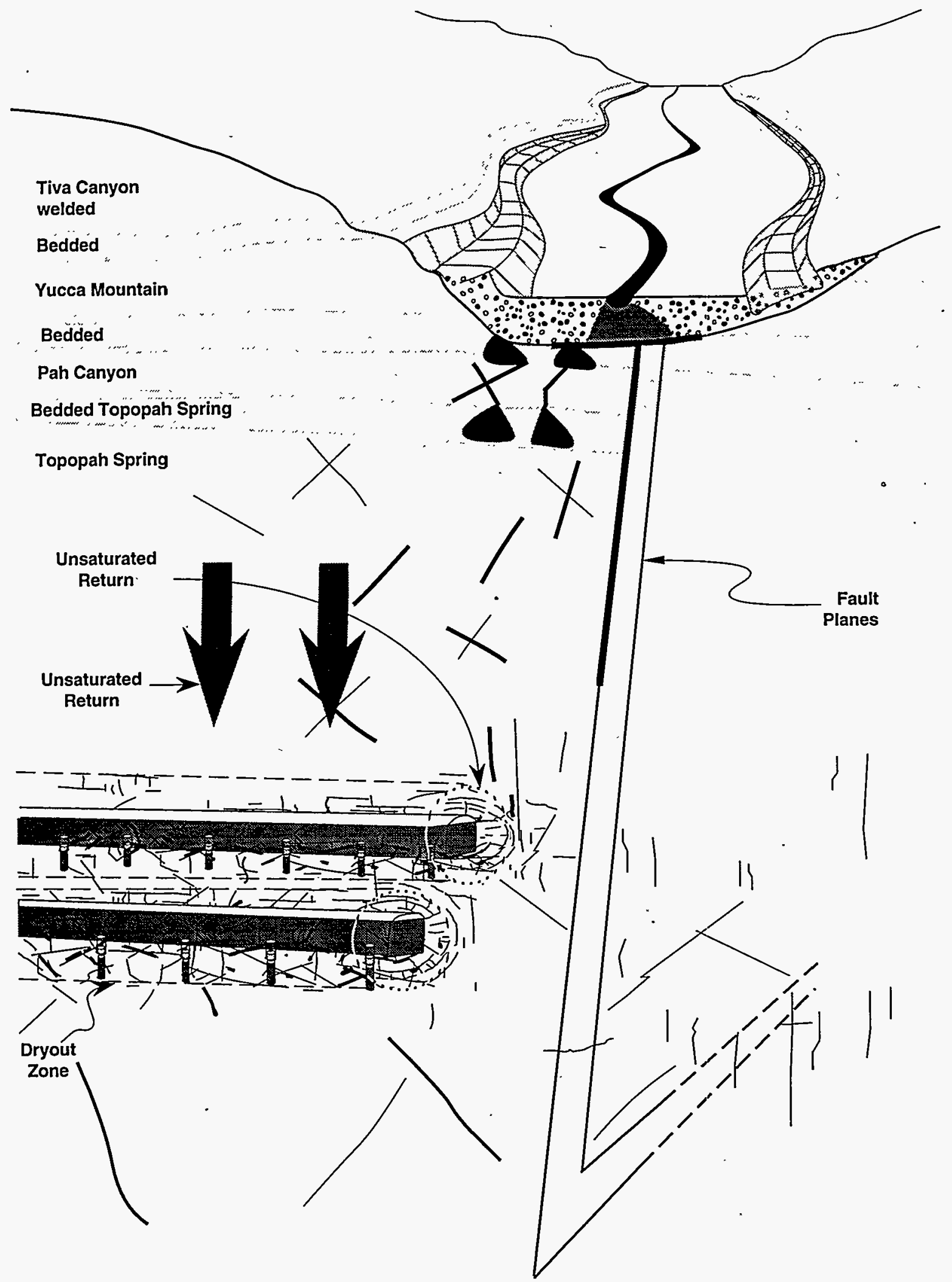

Sketch J2. Large-scale view of the return flow of liquid and vapor to the repository and the interaction with fracture flow from a wash with faults. 


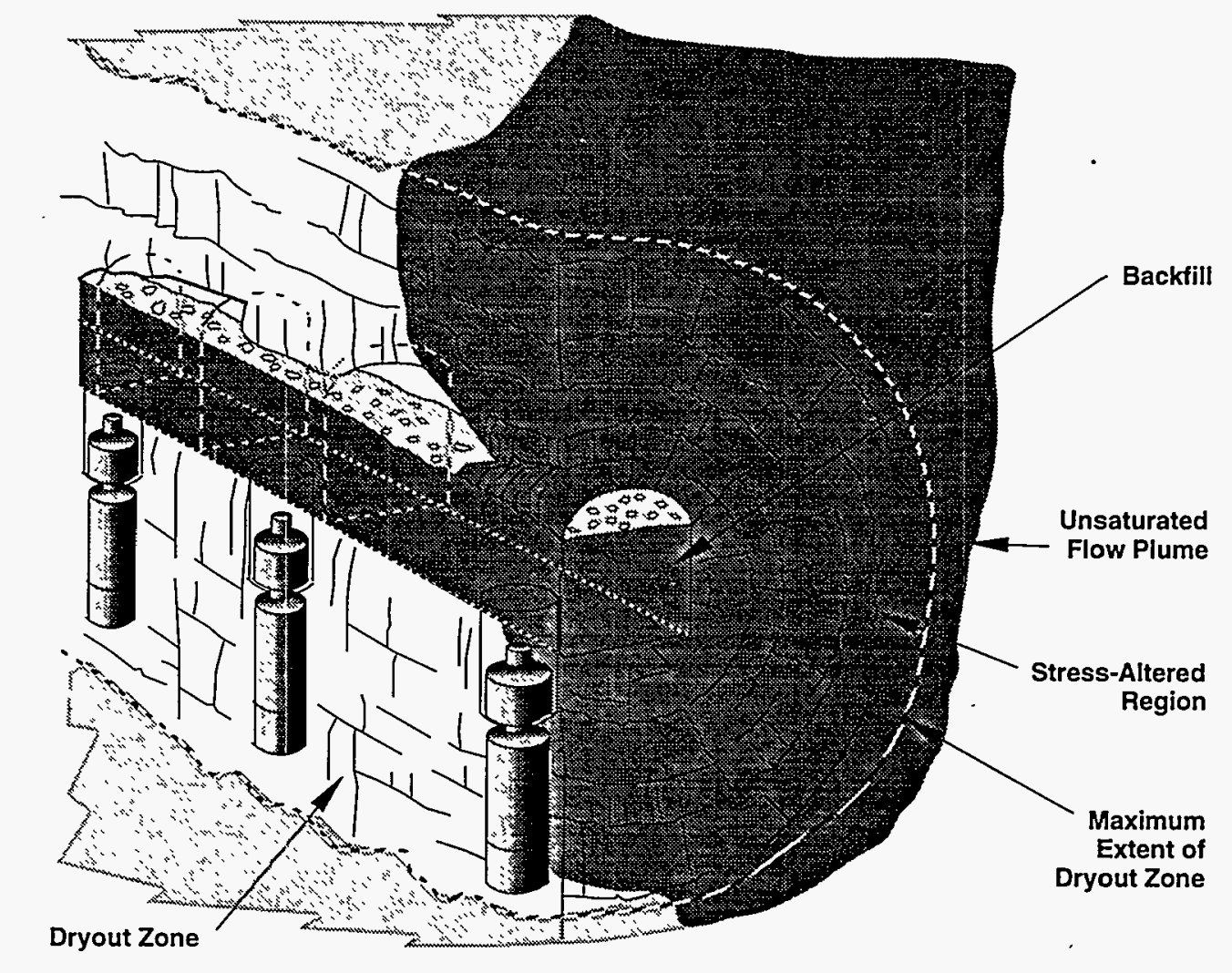

Sketch J5. Episodic unsaturated flow from runoff reaching the waste containers. 


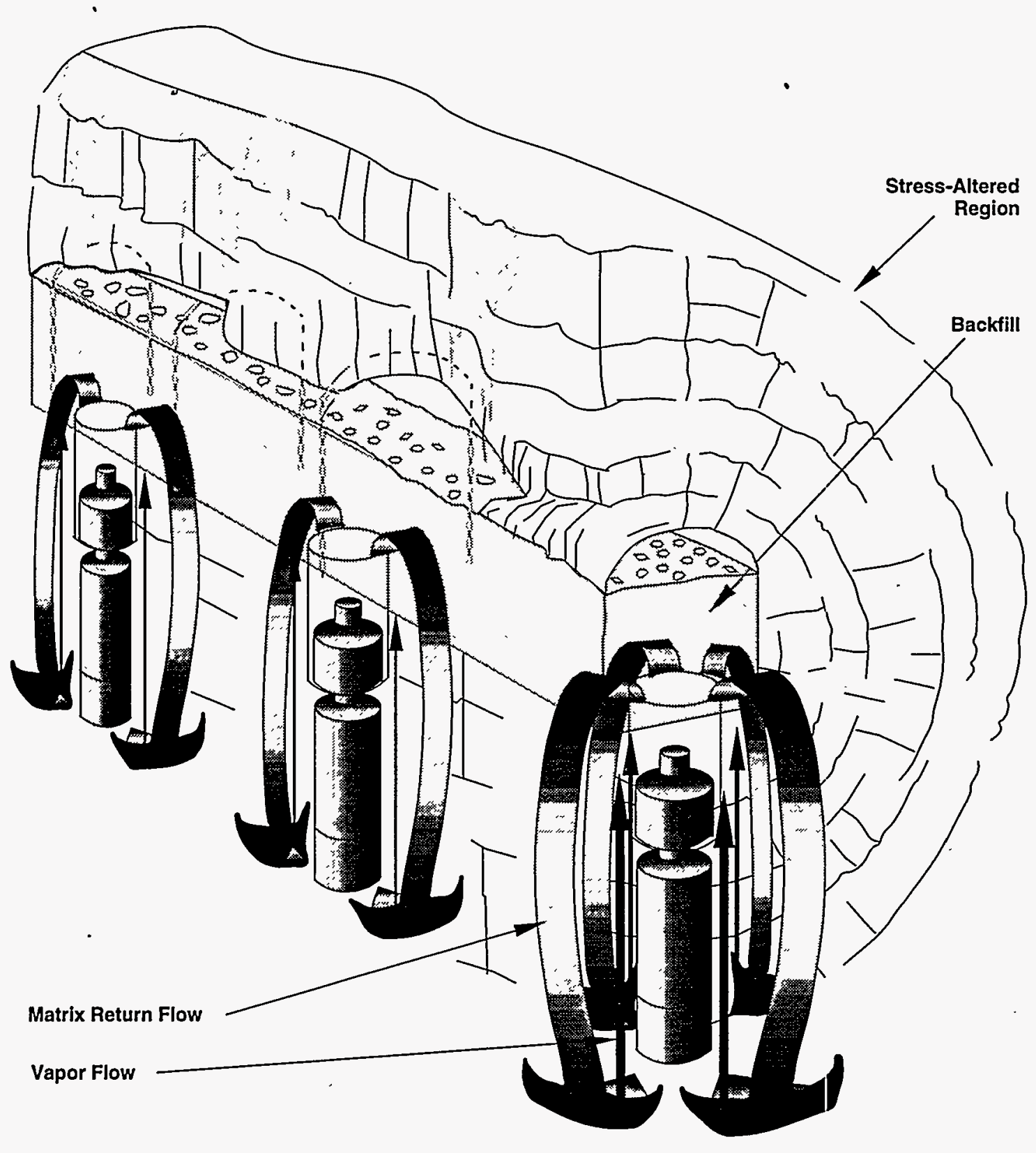

Sketch H2. Details of heat-pipe behavior near the backfilled drift. 


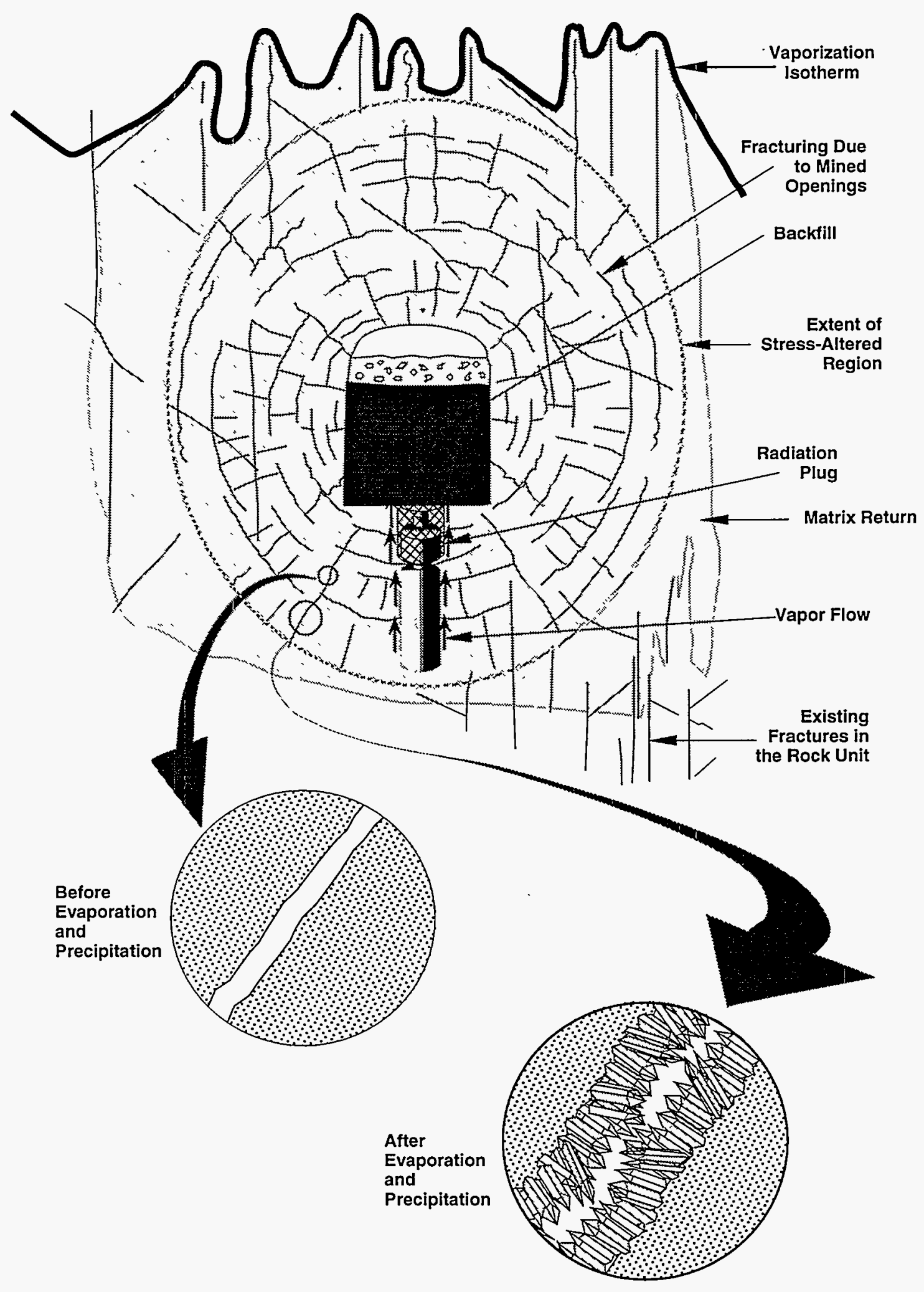

Sketch I2. Vaporization and dryout near the waste container as a result of heat-pipe behavior. The enlargements show the very-small-scale process of alteration of permeability and porosity. 
Mobilization of contaminants begins during the heat-pipe phase and continues as the system cools to allow locally saturated reflux as well as unsaturated/saturated matrix flow. Return flow occurs after cooling (Sketch J2); however, degradation (Sketch K2) has, in this case, been continuous. Unsaturated-flow mobilization requires contact between waste container and rock (Sketch $\mathrm{K} 2$ ). If local conditions at a given waste container allow matrix flow, transport can begin.

After contaminants are mobilized, flow must reach and pass through the Calico Hills units to produce releases. A number of modes of flow are fed by the redistribution of fluids produced by the heat-pipe regime (Sketch M2). They include both locally saturated return to the waste containers and unsaturated flow to the waste containers with unsaturated leaching and transport. Fingering is indicated as a possible mechanism that would allow a complete traverse of the Topopah Spring units, followed by unsaturated flow and transport in the Calico Hills units. One unsaturated flow and transport column is indicated as a plume from a waste container to the water table, partially confined by bounding fractures. Such plumes may persist if a sufficient volume of fluid is available-a circumstance that may only be possible if additional fluid is brought in by fractures connecting to recharge from the wash. Such recharge could also have a profound effect on local heat pipes around waste containers, functioning as a fluid source and as a heat sink. Again, because a wash is the fluid source, influx of fluids is expected to be episodic. Volumetric rates of recharge significant to the repository remain to be calculated. Our tree has led us to construct the scenario described by Sketches C, D, E1, F1, H2, I2, J2, K2, and M2, Path $\widehat{1.4}$.

\section{Fracture Flow Through Bedrock, Cold Repository}

We now consider the "Cold Repository" branches, Paths $\widehat{1.5}$ and $\widehat{1.6}$. The cold repository sees an environment (permeability, porosity, and thermo-mechanical stress) (Figure G, p. 10) altered from that of prerepository conditions, which must be considered in the modeling.

Path $\widehat{1.5}$. Path $\widehat{1.5}$ for the cold repository develops the case that fracture flow through bedrock (Sketch F1) is imbibed into the matrix (Sketch $N$ ) to form an unsaturated flow plume. The plume works its way through the stress-altered region to reach waste containers (Sketch G5). At this point there may be enhanced degradation of the EBS, presuming some physical contact occurs between container and rock wall. Sketch K6 shows such contact occurring because of rock spall and also suggests mobilization as contaminant fingers and in an unsaturated flow plume. Because runoff-producing events are episodic, mobilization could also be episodic if rocks around the repository do not damp out the time dependence. For the case of episodic mobilization, one might expect a contaminant front advancing periodically through the rock. Flow to the Calico Hills units and transport to the water table will similarly proceed in an unsaturated mode, except for locally saturated flow (Sketch M1). Path $\widehat{15}$ is the scenario described by Sketches C, D, E1, F1, N, G5, K6, and M1. 


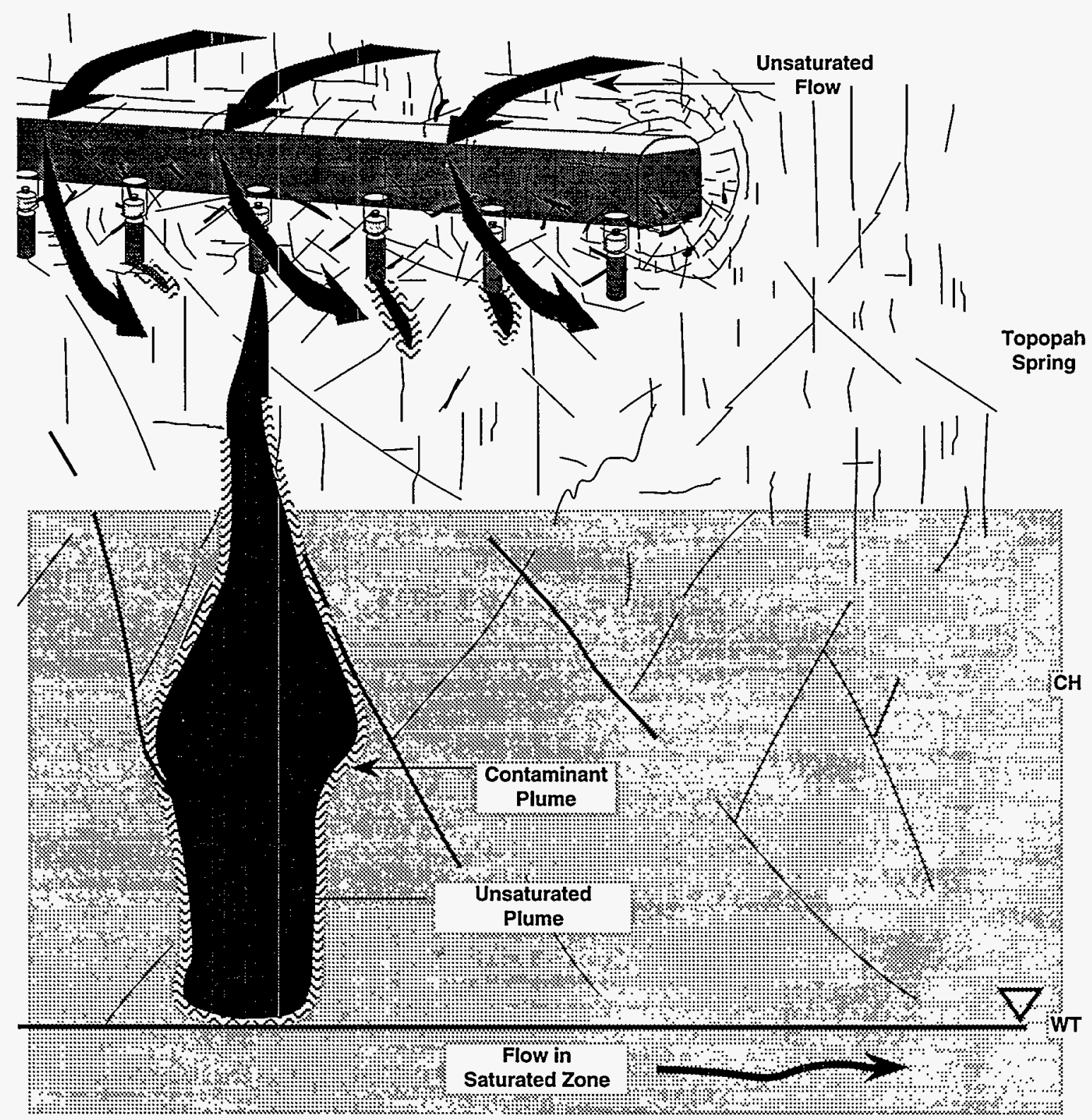

Sketch M2. Transport of contaminants from the repository to the water table, showing unsaturated flow to the waste containers followed by unsaturated transport to the water table. $\mathrm{CH}=$ Calico Hills units; WT = water table. 


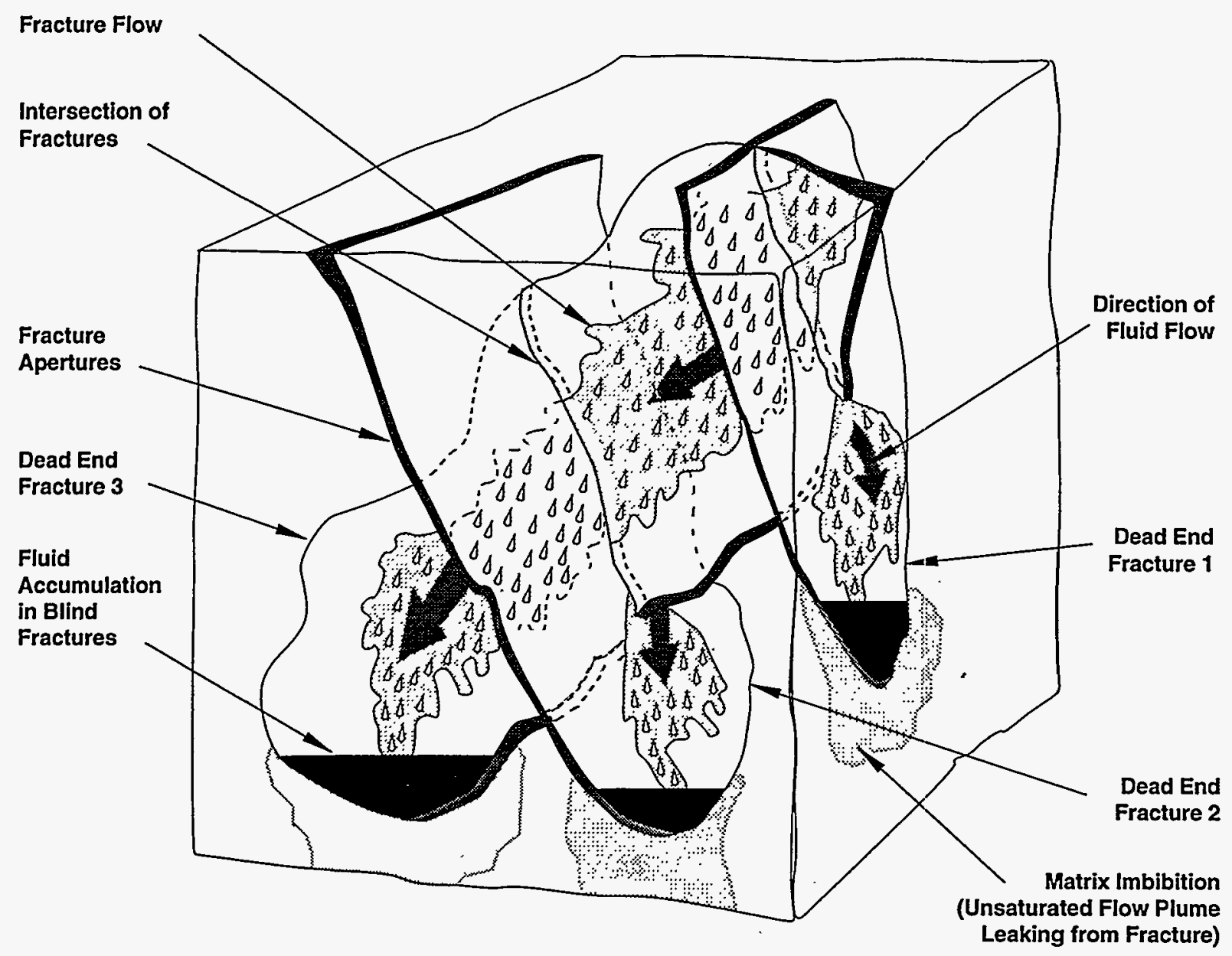

Sketch N. Imbibition of liquids into the matrix from fractures to form an unsaturated-flow plume. Three intersecting fractures feed leakage to the surrounding matrix. 


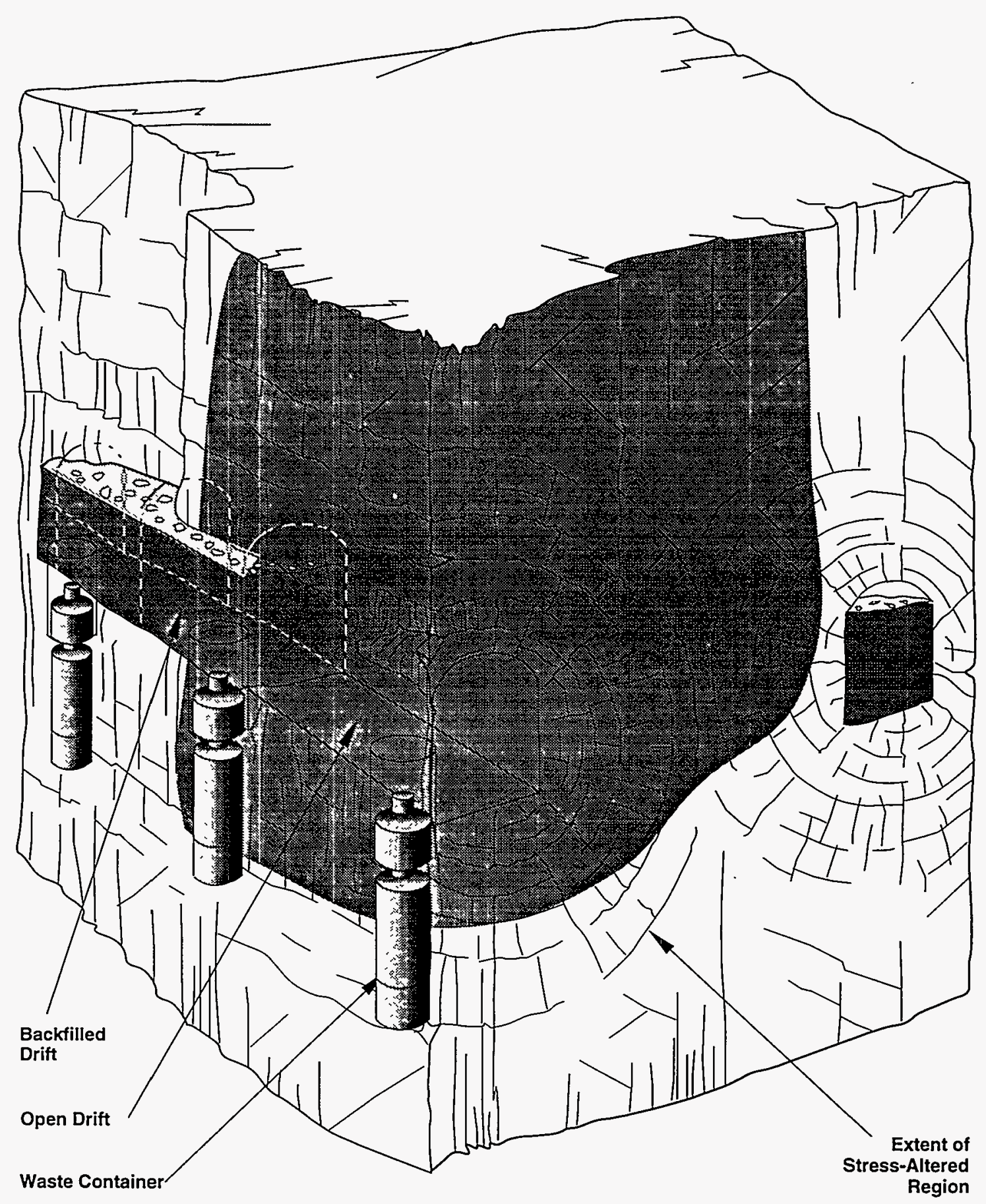

Sketch G5. Unsaturated flow plume entering a cold repository. 


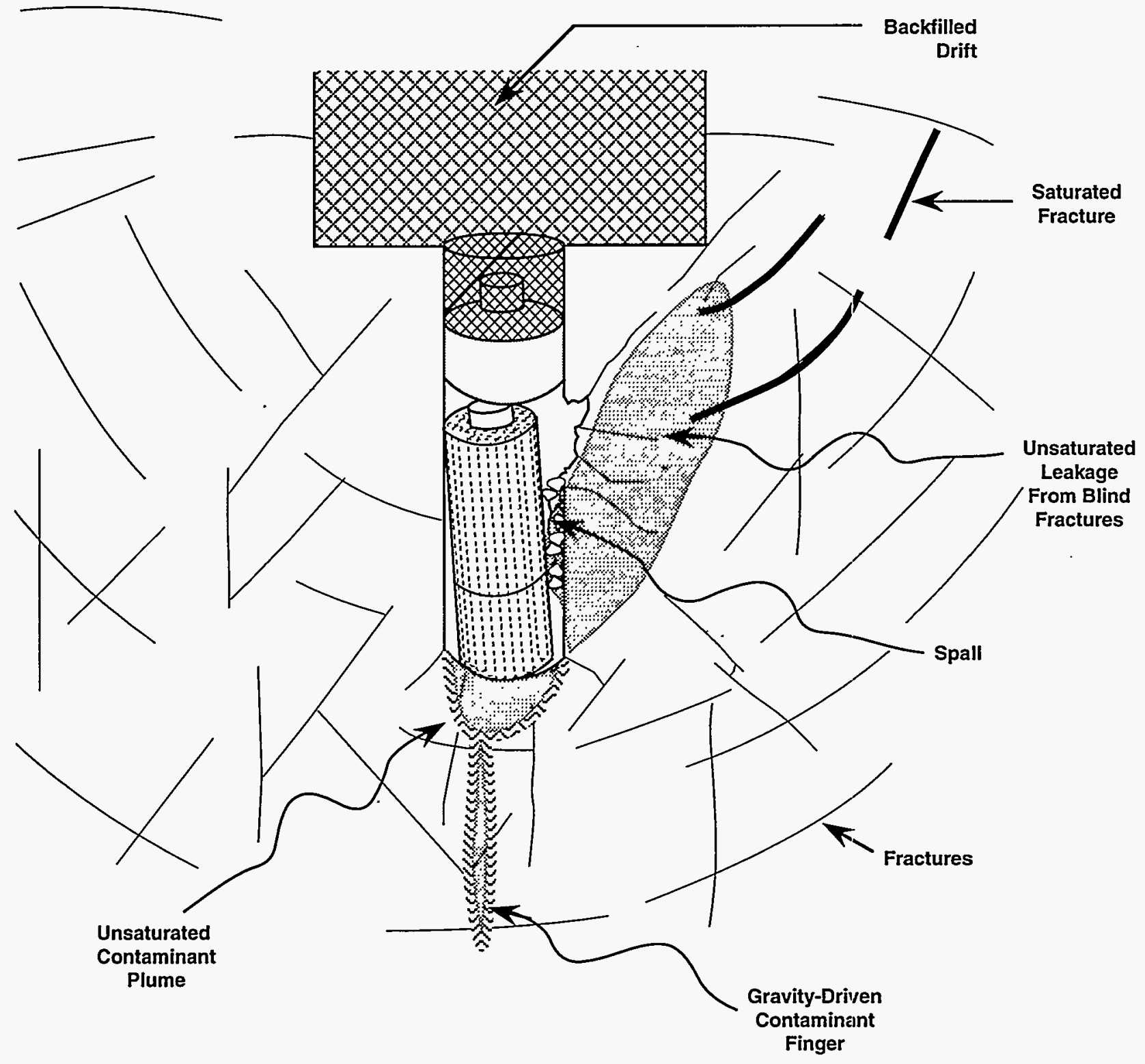

Sketch K6. Degradation of the EBS and mobilization of contaminants in the presence of blind, saturated fractures leaking to the unsaturated flow system. Fractures are three-dimensional and may intersect outside the plane of the sketch. Some contact between rock and container, shown here by rock spall and a leaning container, is necessary. 
Path $\widehat{1.6}$. Path $\widehat{1.6}$ for a cold repository considers fracture flow to the stress-altered region around the openings. This diversion of fluids to the stress-altered region about the repository is indicated in Sketch H3. Substantial lateral migration is required, and the number of waste containers at risk may be small.

The recharge events that are the source of the fluid are episodic. Current studies indicate significant precipitation events must follow each other within a few days for fluid to reach the bottom of the alluvium. Otherwise, two phenomena overwhelm downward movement. The first, evapotranspiration, removes water directly to the atmosphere from alluvium or near-surface rock. The second, near-surface flow of water, may transport water outside the area of interest without the water ever participating in the larger ground-water system.

Water may flow down the alluvial channel beneath the surface or may enter bedrock, depending on the details of the local hydraulic gradient. Fracture flow to the repository-more specifically to the waste-is shown in Sketch 13. The directions and volumetric rates of flow are controlled by the interconnectivity of the fracture network. Lateral migration of polymer drilling fluid over distances of 1000 feet has been observed during well-drilling in this wash (Water, Waste \& Land, Inc., 1986; Whitfield et al., 1990).

Degradation of the container began during the hot period and is reinvigorated here (Sketch K3) by arrival of fluids by fracture flow and unsaturated flow (as leakage from fractures). In Sketch K3 we have tried to illustrate direct movement in a locally saturated fracture as well as leakage to the unsaturated flow system from a conducting fracture. Thus the EBS may be attacked by both saturated and unsaturated fluids. Contaminants can move in the locally saturated fractures leading away from the waste container or they can move by one of the unsaturated transport modes (distributed or as fingers). Flow through the Topopah Spring units and into the Calico Hills units with transport to the water table is shown in the most general sense in Sketch M. Contaminants are mobilized and move through fractures and through the matrix in both units. The fracture properties and fracture density of each unit are important to the details of movement. Since influx from a wash is episodic, contaminants are flushed with each episode, forming a contaminant front that advances slowly toward the water table. With this discussion we have constructed the scenario in Path 1.6 described by Sketches C, D, E1, F1, H3, 13, K3, and M.

\section{Unsaturated Flow in Bedrock, Hot Repository}

The next set of paths for consideration (Tree Segment 2) starts from saturated flow to bedrock (Sketch E1, p. 25) and continues with unsaturated flow in bedrock from the wash bottom to wherever interaction with the repository first occurs (Sketch F2, p. 28). A large, descending unsaturated plume is produced. The alluvium is essentially a saturated sponge leaking to feed this unsaturated plume. We do not expect a steady-state unsaturated plume to form, because the source is episodic and drainage down the wash competes for the available water. Sketch F2 shows a number of strata with differing abilities to maintain such an unsaturated plume. For a sufficiently large unsaturated source, contrasts in hydraulic parameters can force local saturation and fracture flow. If flow in the Topopah Spring units is unsaturated, it is constrained to be generally outside the fractures except for gravity-driven nonequilibrium flow in fractures. Eventually, the descending plume interacts with the repository as unsaturated flow 


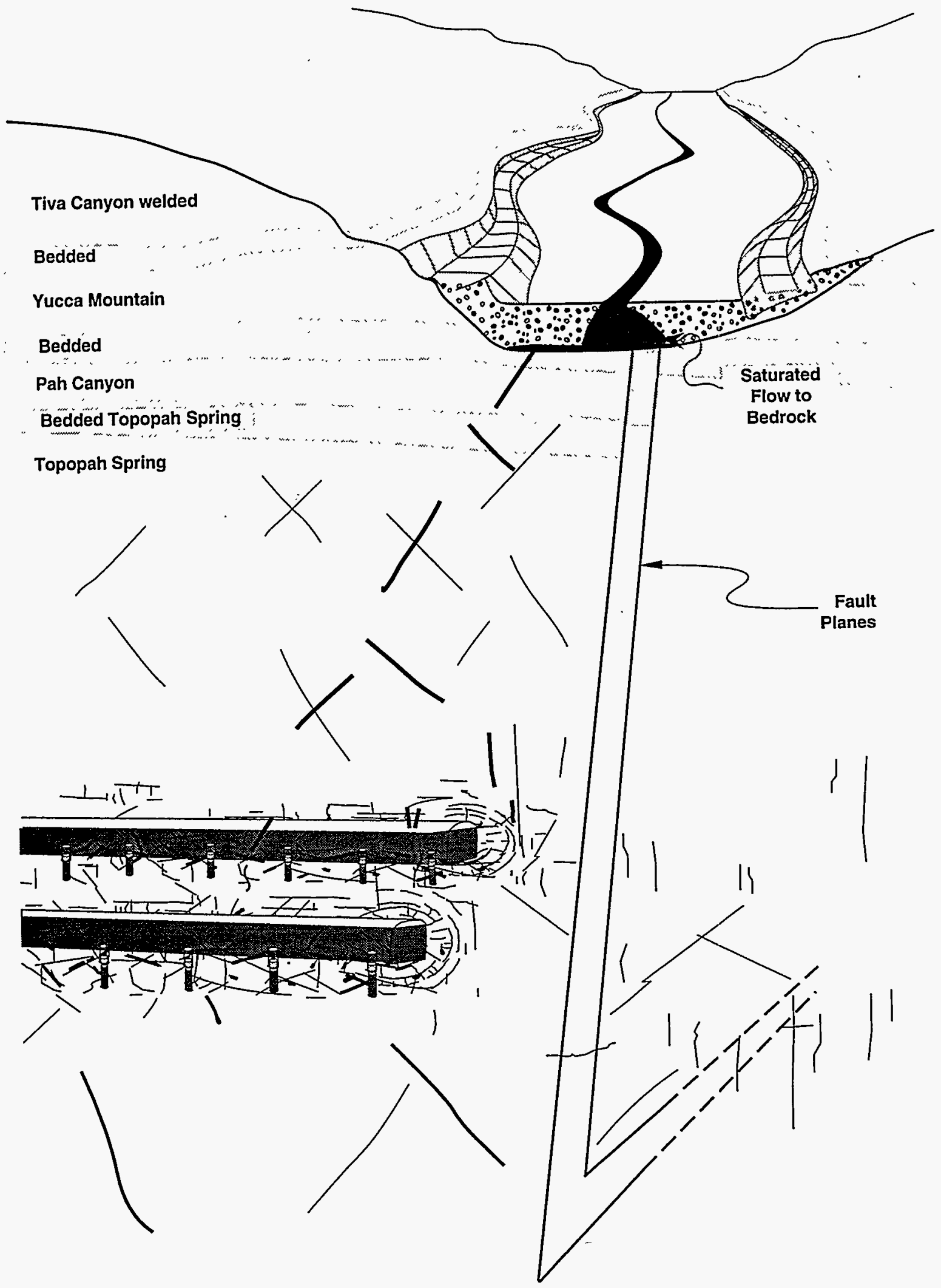

Sketch H3. Saturated flow to bedrock followed by fracture flow to the repository. 


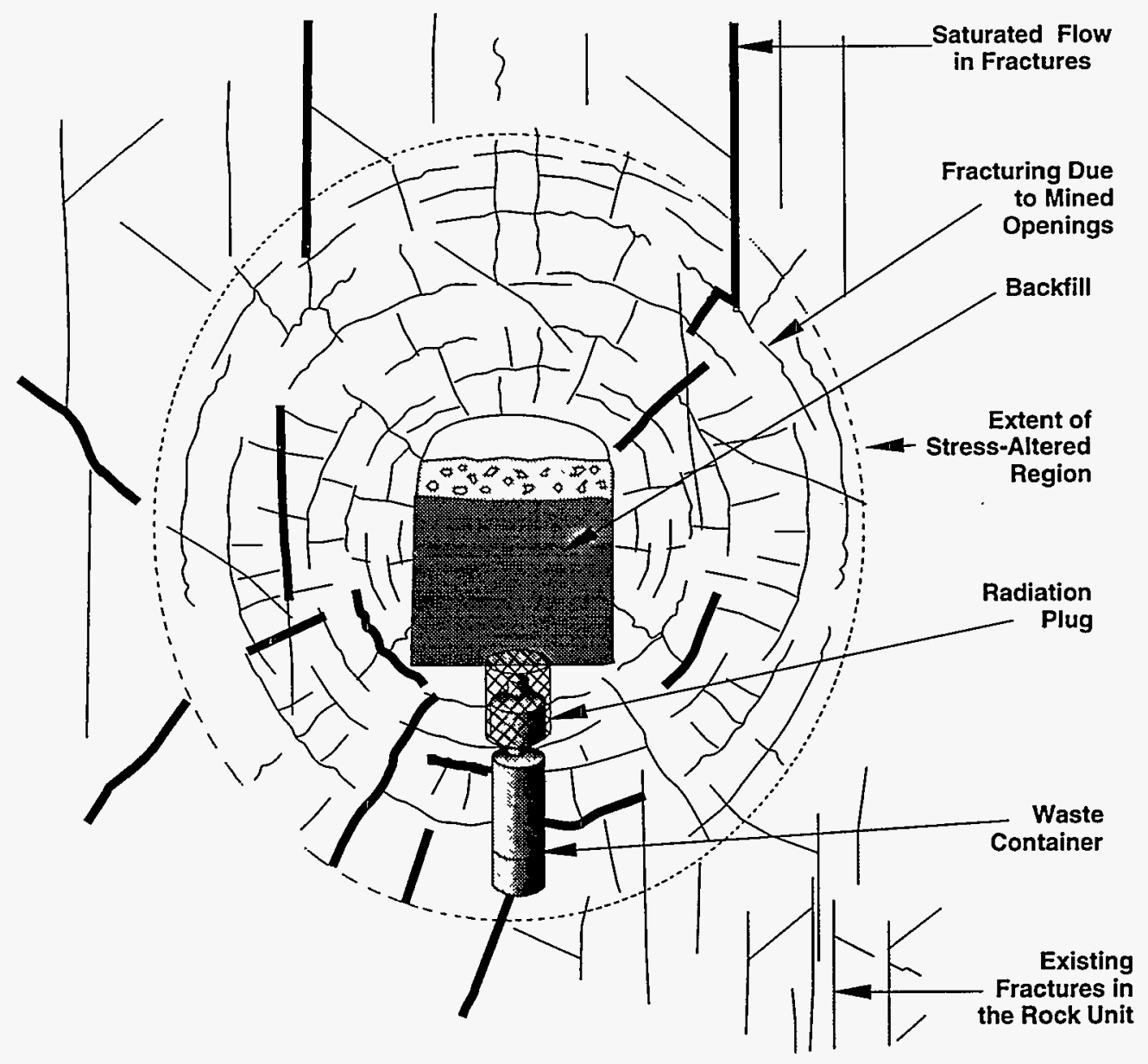

Sketch 13. Fracture flow to the repository. Fractures are three-dimensional and may intersect outside the plane of the sketch. 


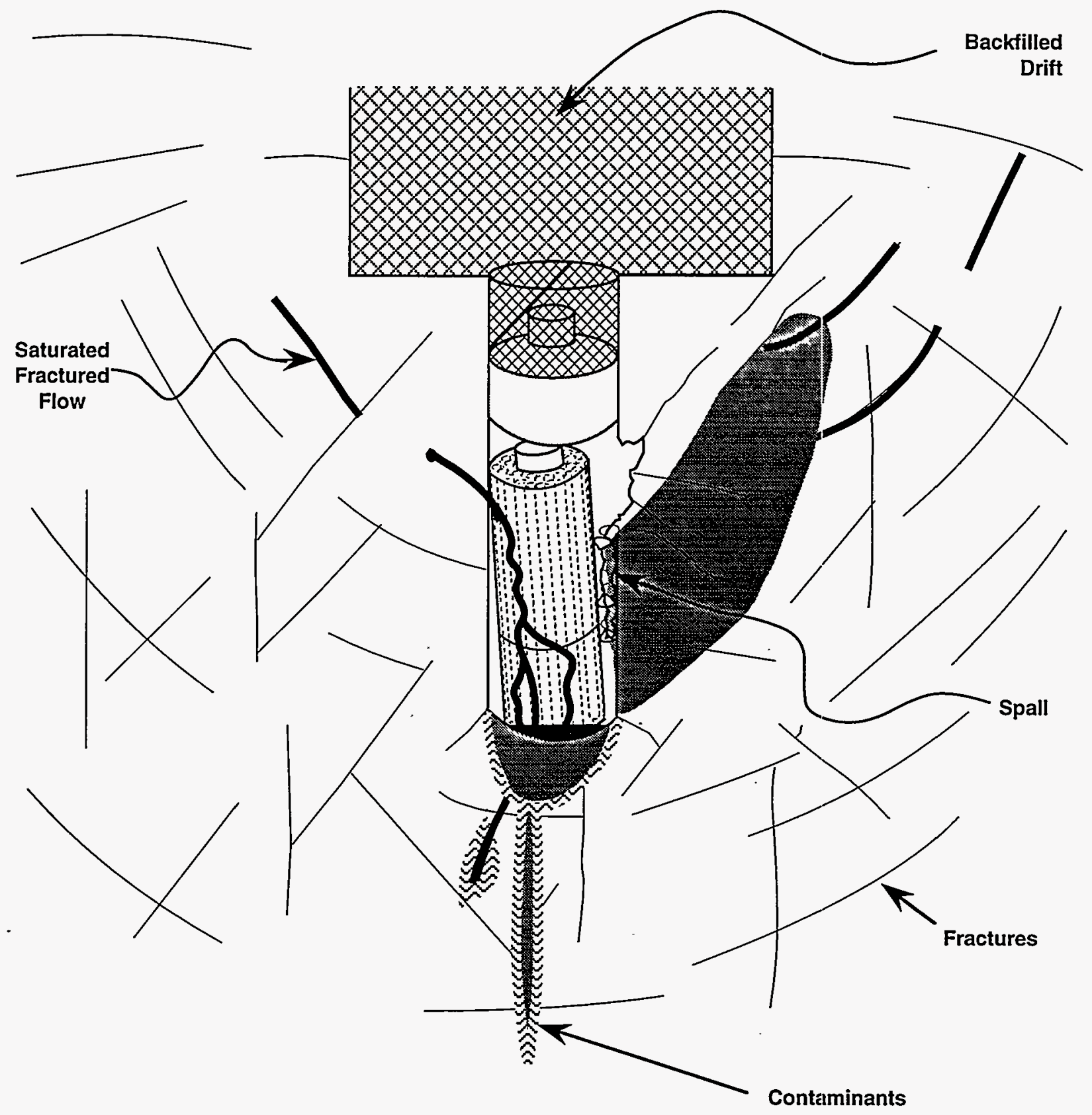

Sketch K3. Degradation of the EBS and mobilization of contaminants, showing both saturated fracture flow and unsaturated flow from blind fractures to the waste container. 


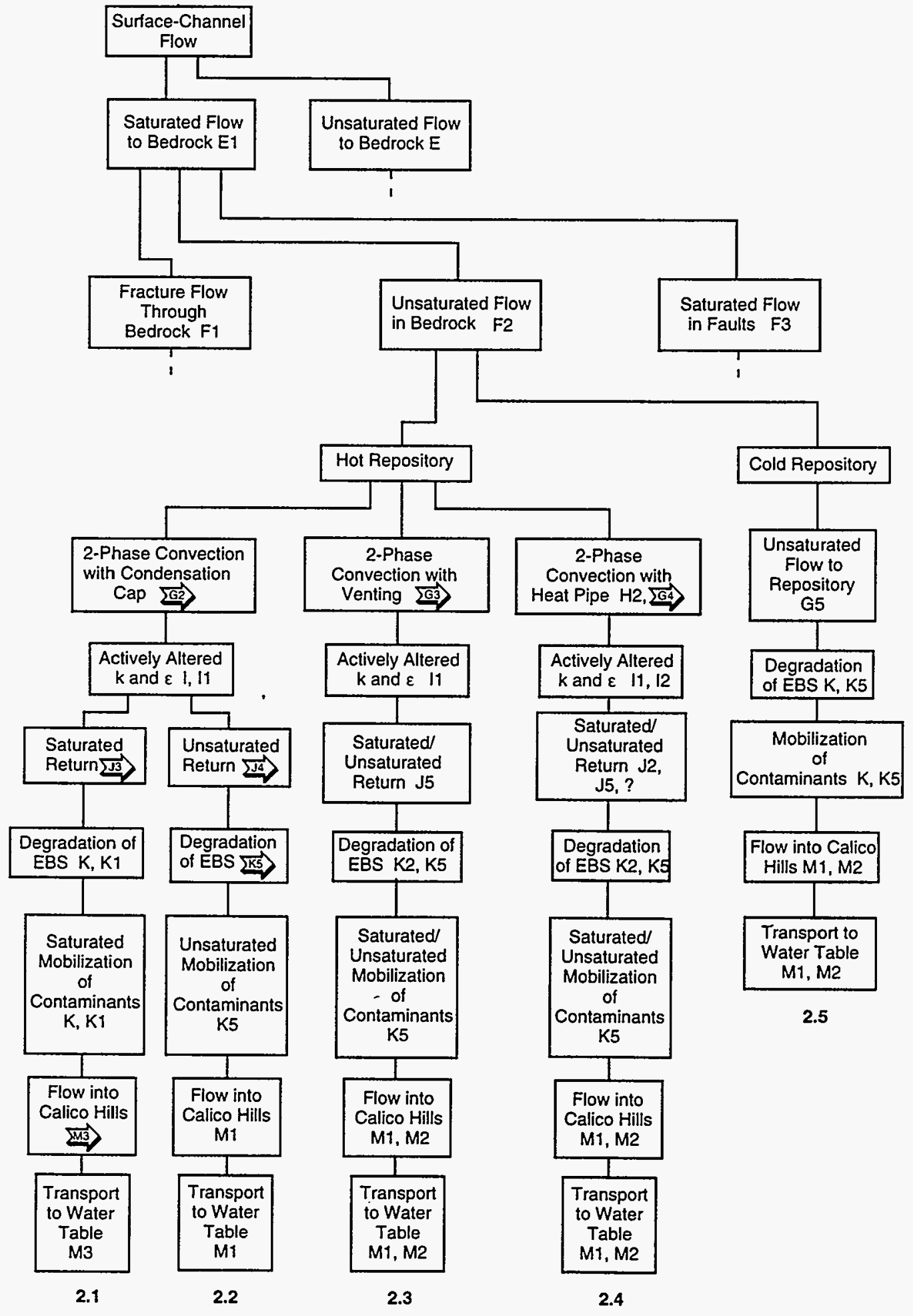

a. Events and their relationships, with cross-references to sketches illustrating each event.

Tree Segment 2. Nominal Flow, Runoff-Producing Events, Runoff to Washes, Washes With Faults, SurfaceChannel Flow, Saturated Flow to Bedrock, Unsaturated Flow in Bedrock. 


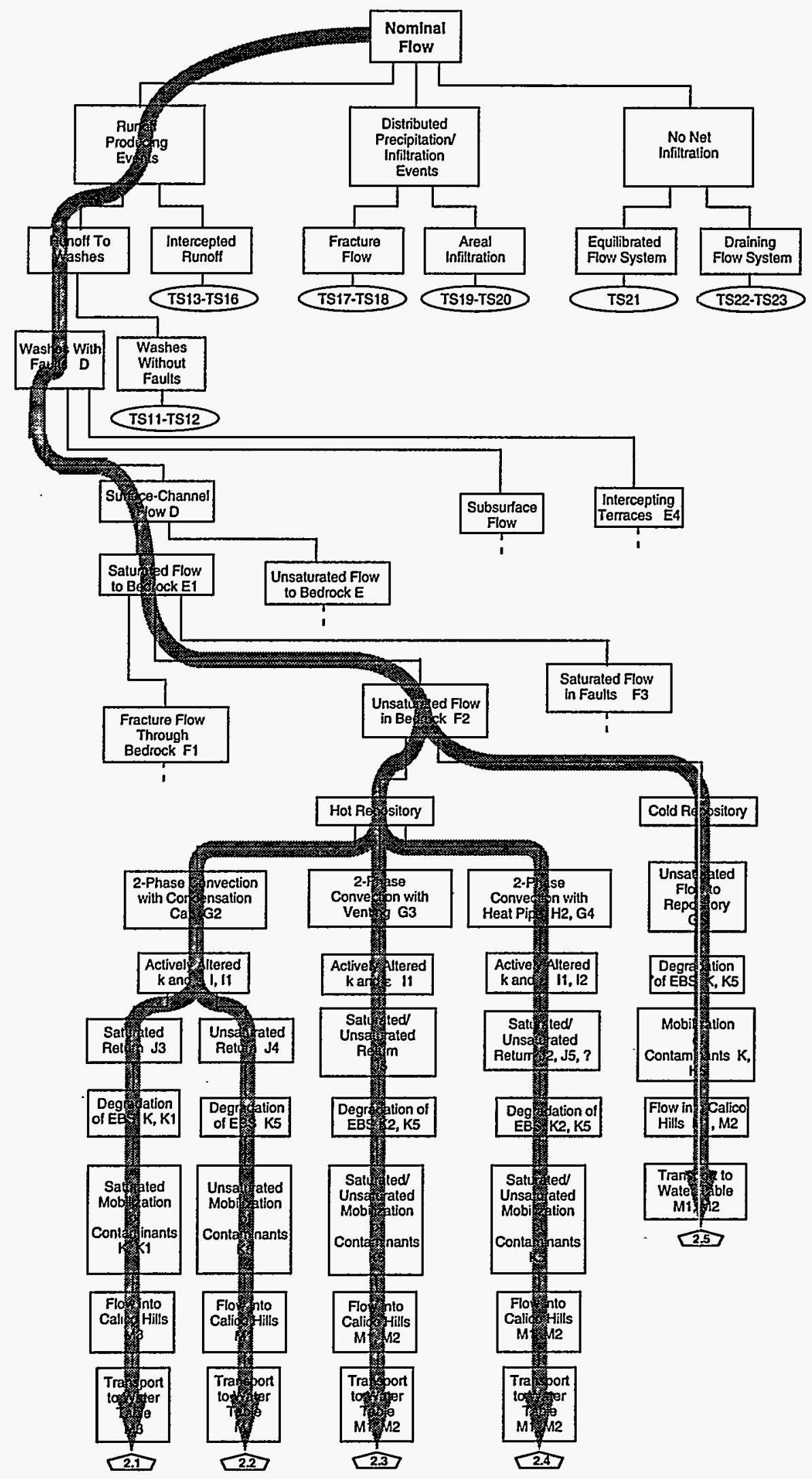

b. Scenario pathways, showing the relationship of the tree segment to the overall event tree for nominal flow. 
arriving in but not filling the matrix. Limiting the flow to the unsaturated system changes the source interaction with the thermal output of the repository because the fluid pulse is a different size and shape and of possibly different duration than for the fracture flow through bedrock discussed earlier. As in the discussion for fracture flow, we distinguish between a hot and cold repository. For the hot repository, we distinguish among three modes (condensation cap, venting, and heat pipe), each involving two-phase flow but with different fates for the condensates. In reality, for a repository of some substantial size, all three modes can be expected in some places at some times.

Path 2.1. Path $\widehat{2.1}$ considers thermal interaction between the repository and the unsaturated flow plume moving downward from a wash with faults in the presence of a condensation cap. Whenever there is wrap-around of a condensation cap-an end effect-it will be altered by arrival of the additional mass of fluid (Sketch G2). One would expect the net effect to be depression of the cap closer to the waste containers and possible increase of saturation at the end of the repository. Such an increase may be sufficient to initiate fracture flow (in the sense of the composite model) to act as a drain around this edge of the facility. In effect such a plume acts as a fluid source and an energy sink. In addition, these episodically available fluids may redissolve precipitates generated by the convective, two-phase system in the manner of the later reflux of the condensation cap, altering permeability and porosity (see Sketches I and I1, pp. 15 and 36).

This brings us to the behavior of the return flow from the condensation cap. First, we will consider saturated return. The condensation cap is a zone of saturation that has been enhanced and depressed close to the end of the repository. A saturated return implies fracture flow (composite model) with flow in a saturated matrix as well. This behavior is sketched in two views in Sketch J3. In this case, matrix flow and fracture flow (composite model) can reach the EBS; matrix flow can occur through the consolidated backfill.

When the container temperature is low enough, fluids reach the waste container through the saturated matrix and the fractures (composite model) (Sketches $\mathrm{K}$ and $\mathrm{K} 1$ ). Localized seepage may occur in the emplacement hole. These fluids attack the waste container, which may have already been breached during the hot period, and mobilize contaminants they reach. The reflux, augmented by the episodic unsaturated flow plume from a wash, moves the contaminants in a saturated-flow mode away from the emplacement hole downward in the Topopah Spring units. Continuation as a contaminated saturated plume depends on the hydraulic details of the Topopah Spring units and Calico Hills units. The transport and flow behavior is suggested in Sketch M3. It is not clear that saturated matrix flow will persist over the distances to the water table suggested by Sketch M3 because of the change from welded to non-welded units. Path 2.1 is the scenario described by Sketches C, D, E1, F2, G2, I, I1, J3, K, K1, and M3.

Path 2.2 . Alternatively, the reflux of the condensation cap could occur as unsaturated flow (Path $\widehat{2.2}$ ), that is, as leakage from the cap that never reaches saturation. In such case, the reflux does not occur in fractures.

There must be some contact of waste container with rock to bridge the air gap and allow fluid to reach the waste container. This return flow (unsaturated reflux) is indicated schematically in Sketch J4. The EBS degrades 

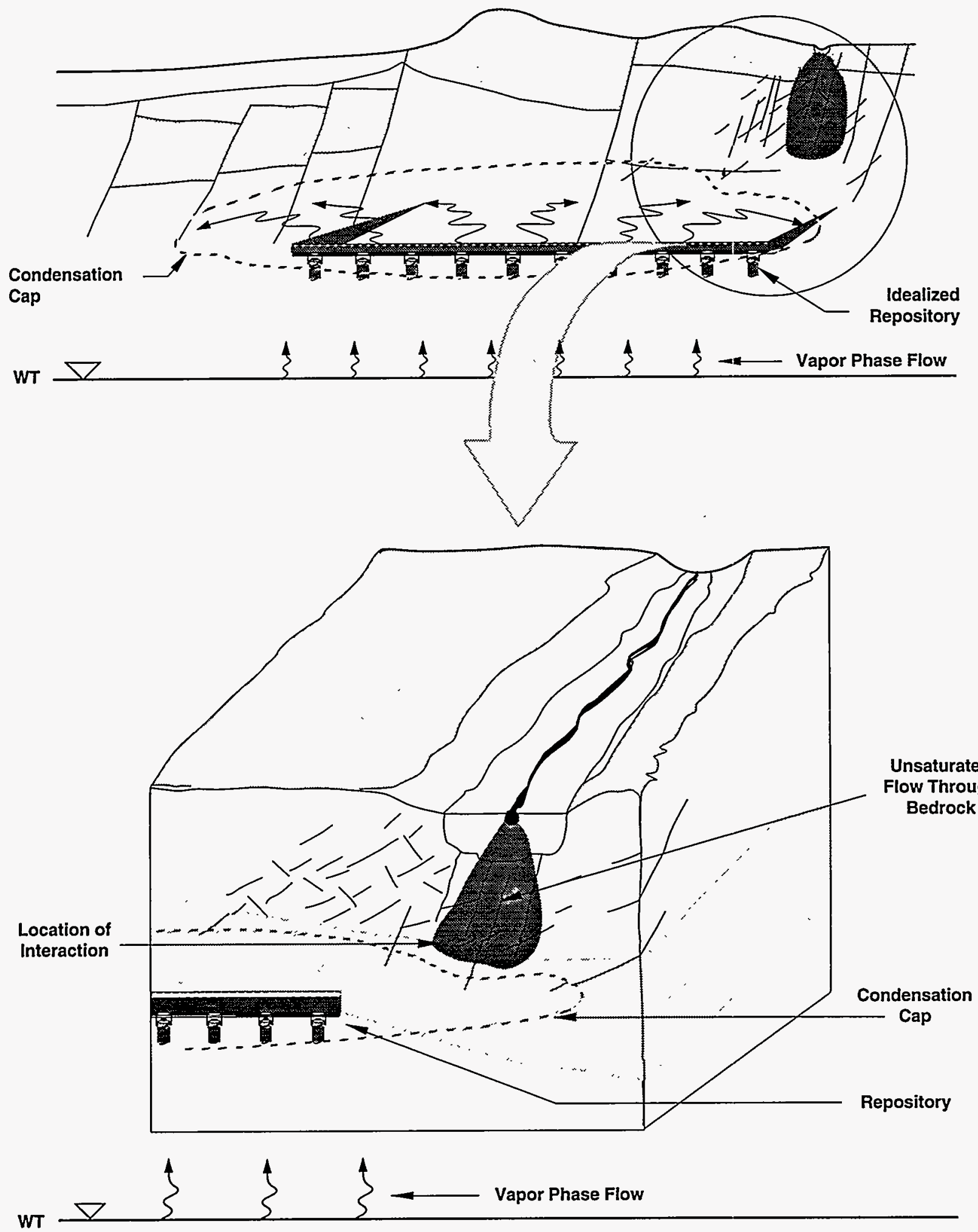

Sketch G2. Interaction between the condensation cap and an unsaturated flow plume descending from a wash. 


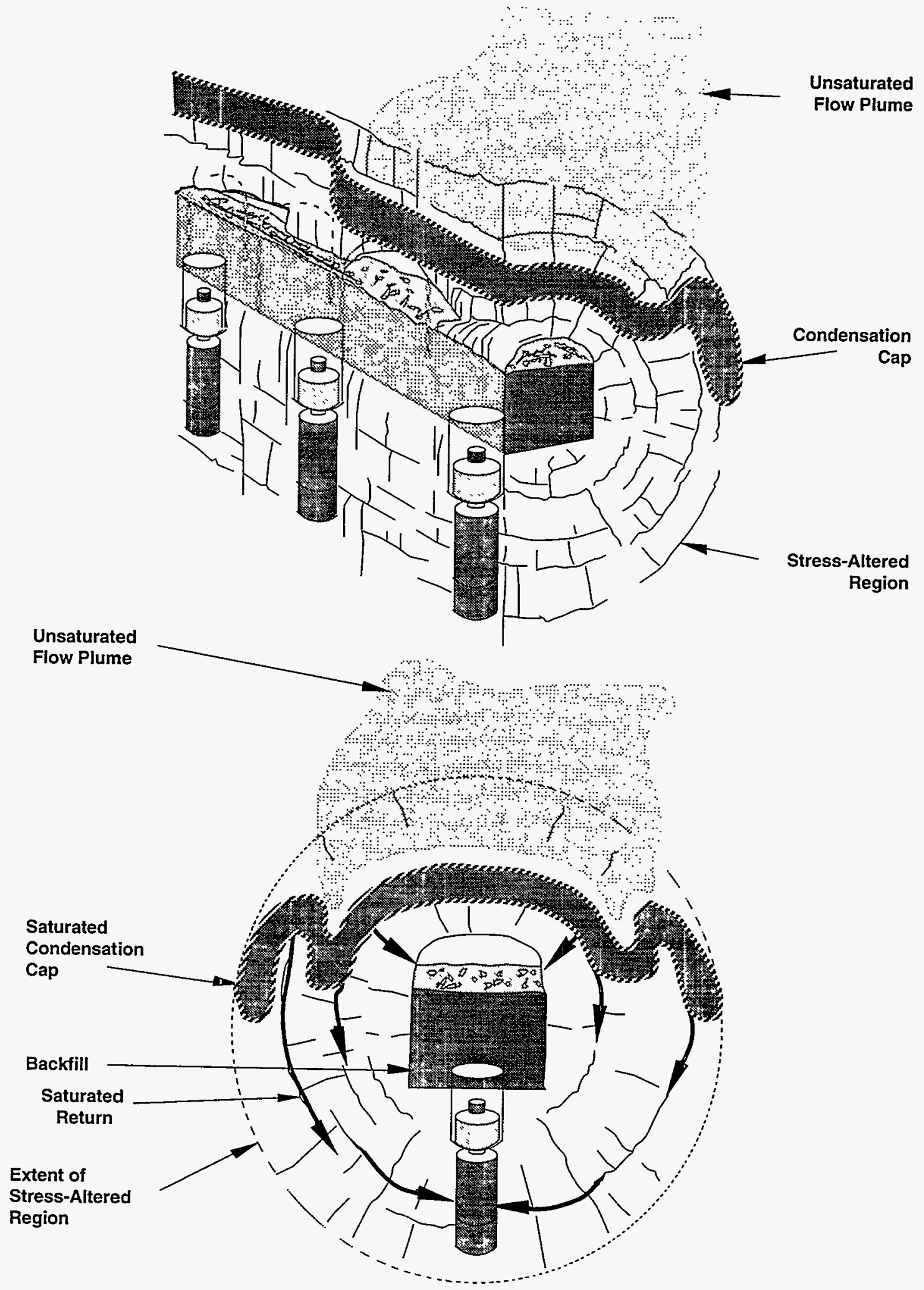

Sketch J3. Saturated return flow from the condensation cap to the waste container in the presence of an unsaturated-flow plume from a wash with faults. 


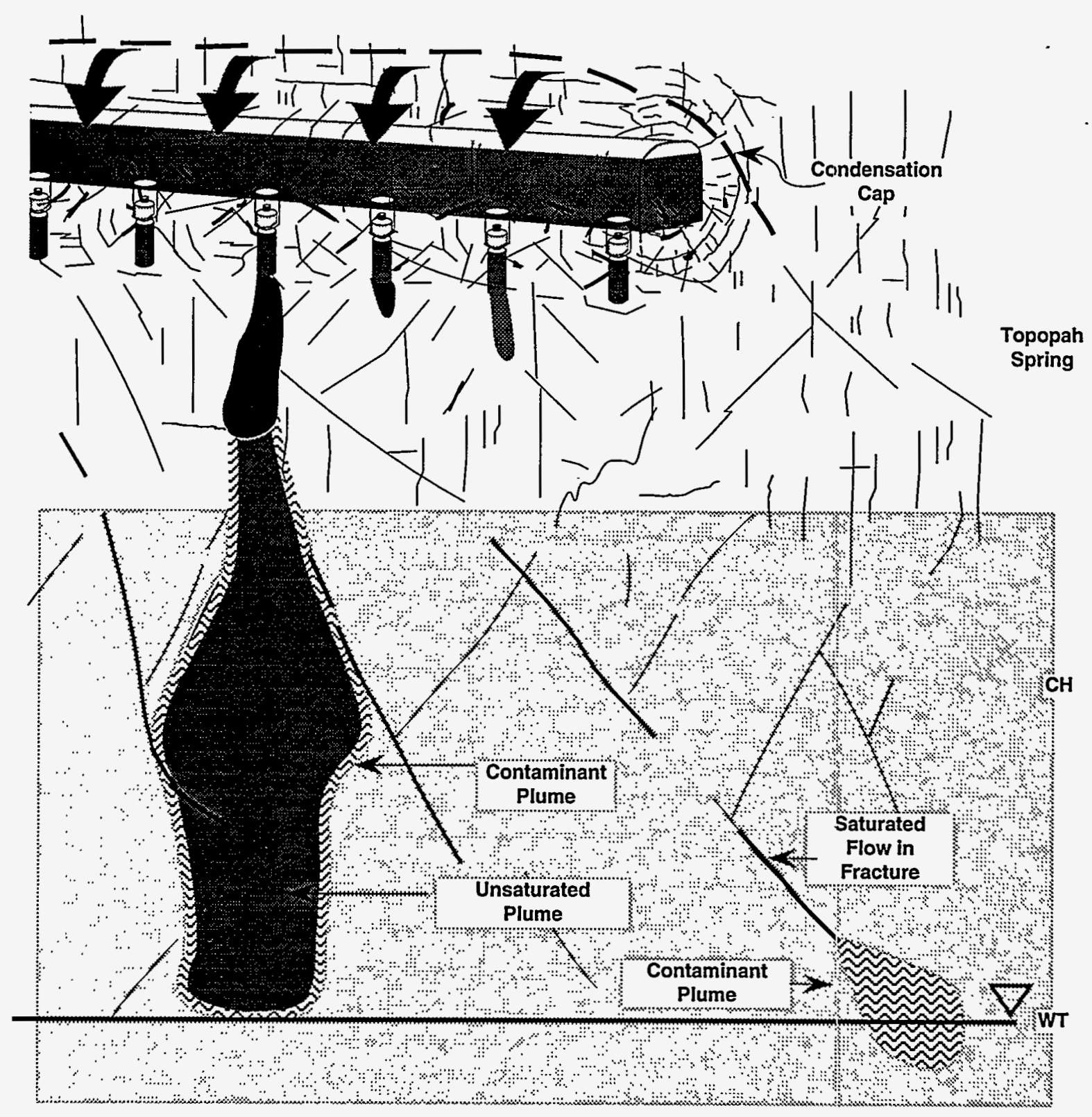

Sketch M3. Transport of contaminants from the repository to the water table, showing reflux from the condensation cap to the repository followed by both saturated and unsaturated transport to the water table. 


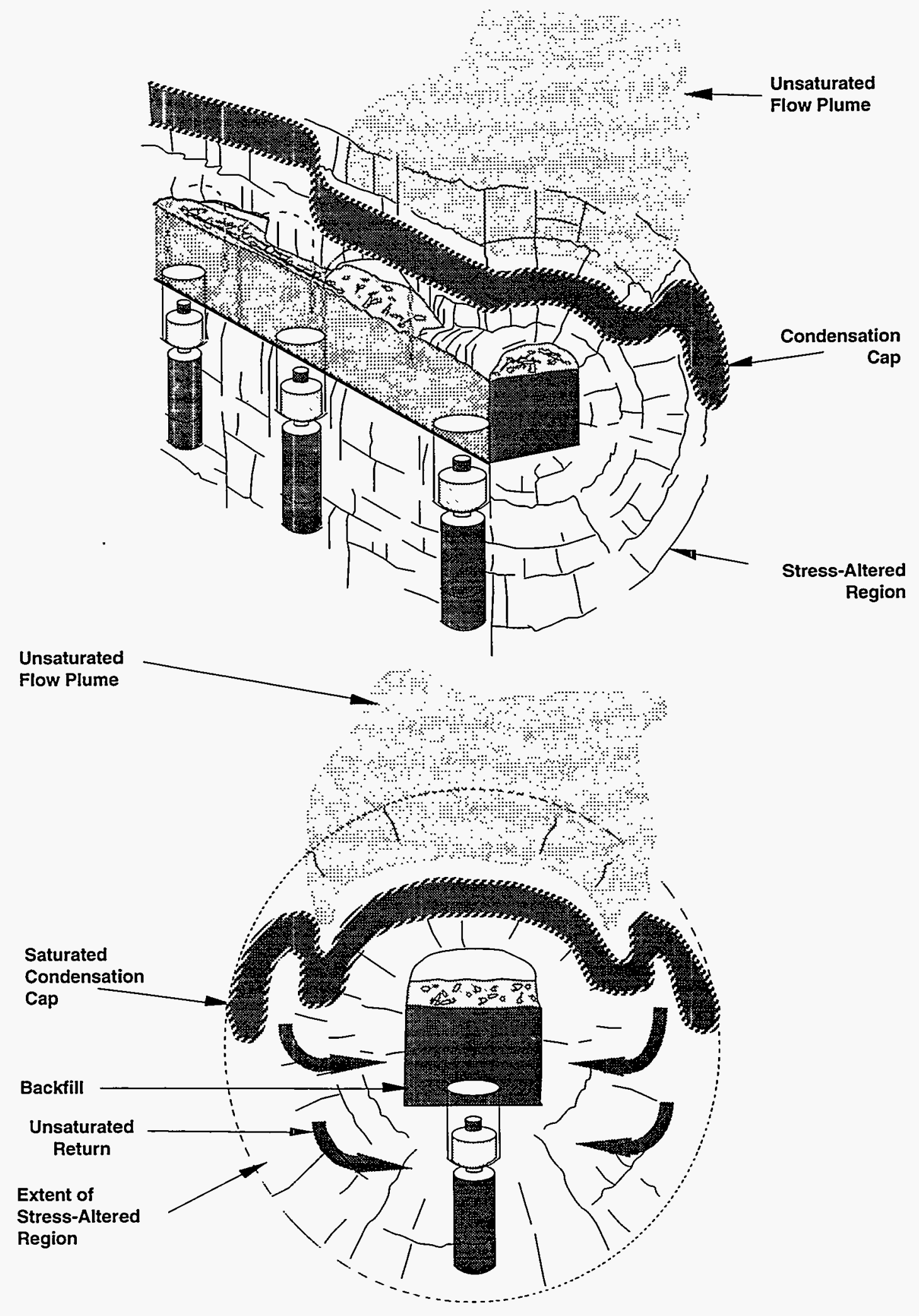

Sketch J4. Unsaturated return flow from the condensation cap to the waste container. 
and contaminants are mobilized in an unsaturated flow field. Some contact between waste container and rock is necessary for liquid water to reach the waste container and waste (vapor is presumed to be ubiquitous). Thermal and mechanical spall are possible (Sketch K5). Mobilization away from the waste container could occur by fingering or by unsaturated-flow transport as suggested in Sketch K5. Whether local zones of saturation can form in this unsaturated system is an open question to be resolved by experiments and modeling. Flow into the Calico Hills units is presumed to be unsaturated and to continue to the water table (Sketch M1). Path 2.2 develops the scenario described by Sketches C, D, E1, F2, G2, I, I1, J4, K5, and M1.

Path $\widehat{2.3}$. The next branch for a hot repository with an unsaturated flow plume as source considers twophase convection with venting of vapor, Path 2.3 . In this case, If two-phase convection occurs with venting, water is removed from the rock around the repository and vented to the atmosphere. Unless the water table acts as a continuous source of replenishment by participating in this two-phase convective flow, there will be dryoutreduction in degree of saturation-locally around the repository. The episodic, unsaturated flow plume that descends from a wash with faults passes through this dry zone, acting as a source of new fluid to be vented during the hot period and as recharge at other times (Sketch G3). Dryout alters rock permeability and porosity by precipitation and alteration of minerals (Sketch I1). The unsaturated return, i.e., the unsaturated flow plume, can persist only when the temperature is low enough for liquids to exist. The plume can arrive at waste containers only if the volume of fluid is large enough to cool the medium below vaporization. Both circumstances allow episodic flushing of contaminants below the waste containers (Sketches J2, J5). Presumably EBS degradation began during the early part of heating when water vapor had access to the waste containers and continues during the return of fluid in the unsaturated plume. Degradation and mobilization are shown in idealized form in Sketches K2 and K5. Unsaturated transport through the Topopah Spring and Calico Hills units will continue to the water table (Sketch M1 or M2), although progress of the contaminants may be episodic, corresponding to the episodic behavior of the unsaturated plume. Path $\widehat{2.3}$ encompasses the scenario described by Sketches C, D, E1, F2, G3, I1, J5, K2, K5, $\mathrm{M} 1$, and M2.

Path 2.4. The third branch for a hot repository fed by unsaturated flow in bedrock at a wash with faults, Path $\widehat{2.4}$, encompasses two-phase convection with a heat pipe.

A heat pipe regime around waste containers implies that there is a two-phase convective cell with vapor transport in fractures and return capillary flow in the matrix (Sketches H2, p. 49, and G4). We expect that the void space around a waste container participates as an equivalent fracture and that a waste container could be exposed to a continuous flux of water vapor (wet steam). Hydraulic parameters change continuously during the functioning of the heat-pipe regime (Sketches I1 and I2). Path 2.4 continues with an unsaturated/saturated return (Sketch J2). As the container cools, the heat pipe may itself bring fluids back to the vicinity of the container. This contribution to the return is not shown as a separate sketch but merely indicate an element as a "?" in Path 2.4 . When the heat pipes shut down, the rock resaturates with fluid from three sources: the episodic unsaturated plume (Sketch J5), 


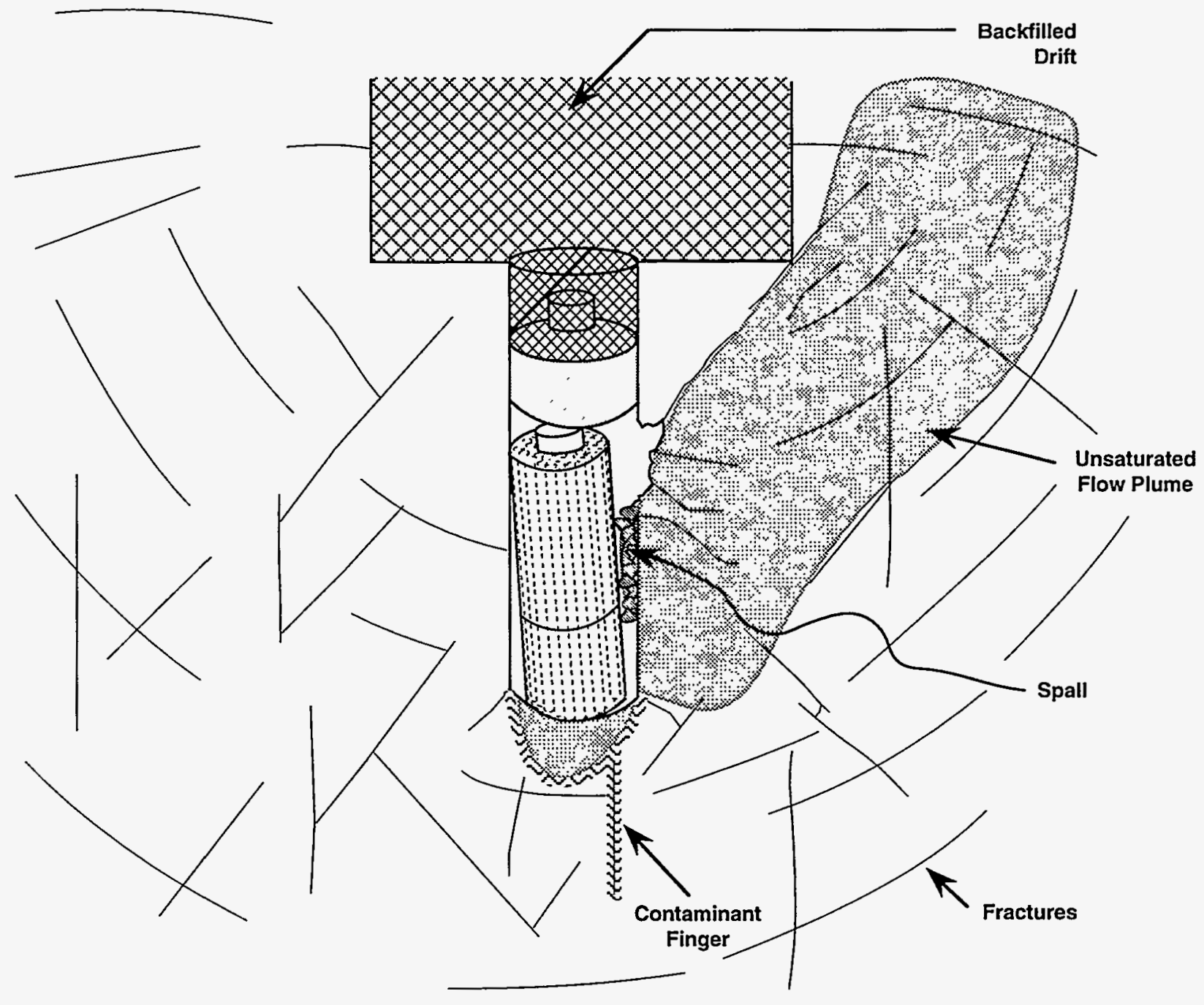

Sketch K5. Degradation of the EBS and mobilization of contaminants in an unsaturated flow field that leads to an unsaturated contaminant plume. Degradation and mobilization require some physical contact between rock and container, shown by spall from the rock wall and by the container leaning against the wall. 

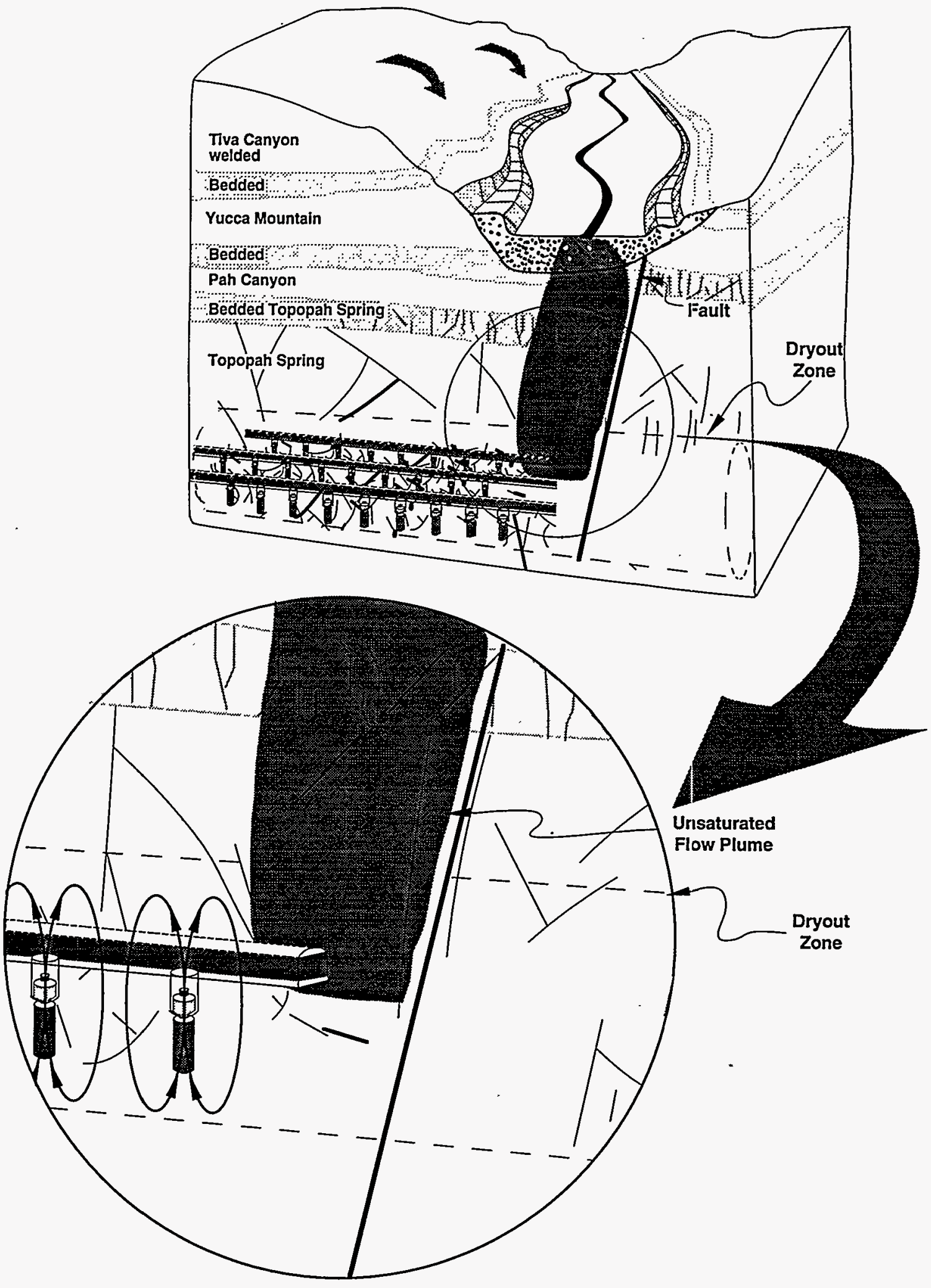

Sketch G3. Unsaturated flow plume interacting with the dryout zone. 


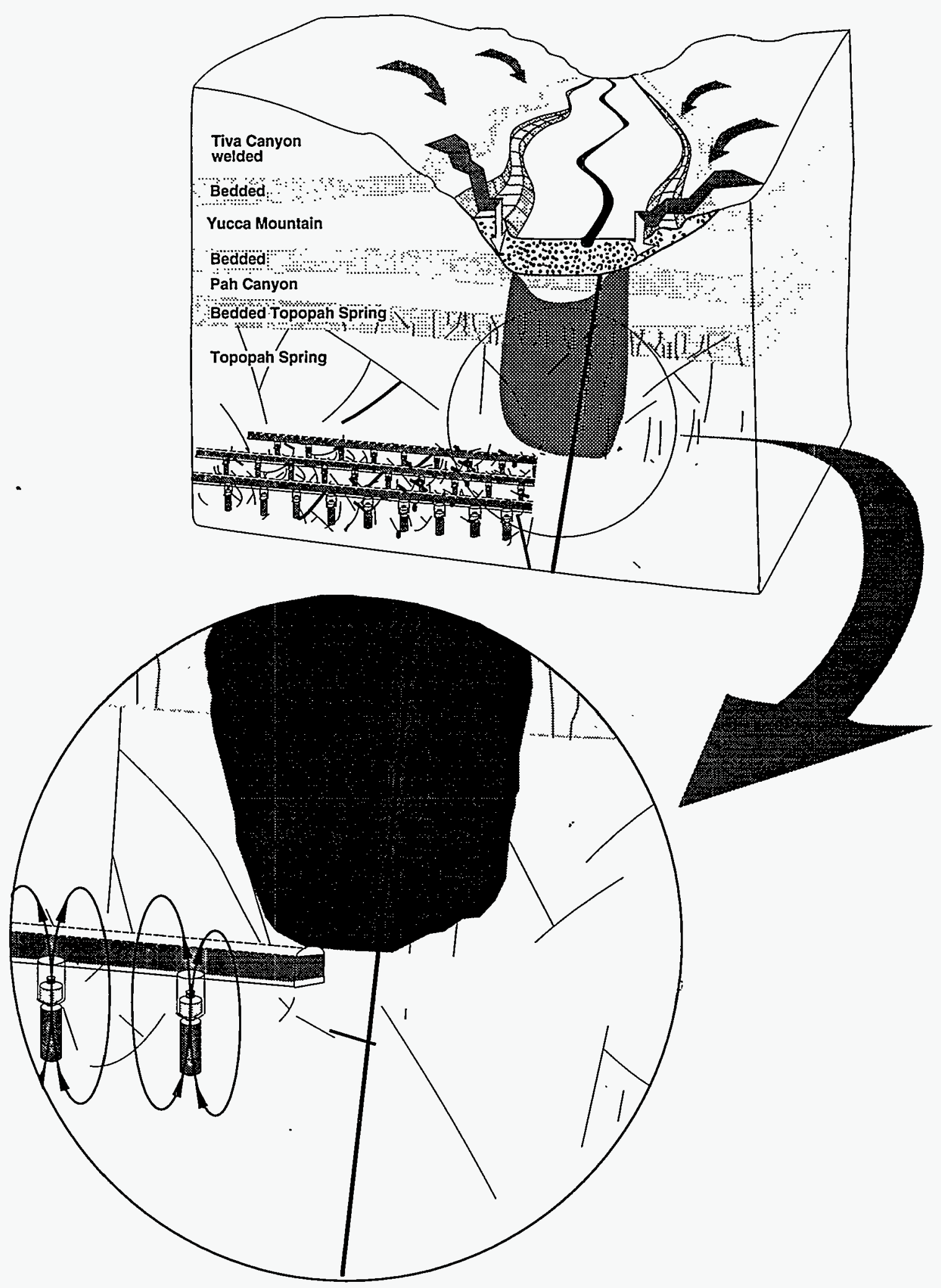

Sketch G4. Interaction of heat pipe around waste containers, with two-phase convective flow and capillary return flow in the matrix, with the unsaturated-flow plume. 
rock outside the region participating in heat pipes, and vapor from rock elsewhere in the mountain (Sketch K2). Reaction of the EBS with the water vapor should cause continual degradation during the thermal period. The influx of fluid in the unsaturated flow plume is superimposed on the heat pipe regime. This influx depresses the upper end of the heat-pipe convective cell by increasing saturation and reducing temperature. For enough flow or low-enough driving temperature, the plume could reach the waste container (essentially extinguishing the heat pipe) and episodically flush contaminants away from the EBS (Sketch K5). Path 2.4 leads us to the construction of the scenario defined by Sketches C, D, E1, F2, H2, G4, I1, I2, J2, J5, ?, K2, K5, M1, and M2.

\section{Unsaturated Flow in Bedrock, Cold Repository; Path $\widehat{2.5}$}

Path 2.5 describes a cold repository receiving an unsaturated flow plume from a wash with faults (Sketch G5, p. 54). In this case, although the hydraulic parameters were altered during the hot phase, the unsaturated-flow plume is otherwise unaffected by residual heating from the repository except that the temperature dependence of hydraulic parameters must be considered. A cold repository is still above the pre-repository ambient temperature even at 10,000 years. Backfill with small-enough pores will participate as a medium for flow, and localized saturation could occur depending on specific values of hydraulic parameters, locally and in contrasting units. Degradation of the EBS began at emplacement and will continue when fluids from the unsaturated-flow plume reach the waste containers. Sketches $\mathrm{K}$ and $\mathrm{K} 5$ again illustrate both degradation and mobilization of contaminants. The mode of flow to the Calico Hills units is presumed to be a continuation of the unsaturated-flow plume and continuation to the water table through the Calico Hills is also assumed to be unsaturated (Sketch M1). If such flow can continue in this way, episodic flushing of contaminants may occur. Episodic flushing results if plumes that do not reach the water table are followed by plumes that do. Path 2.5 develops the scenario described by Sketches $C$, $\mathrm{D}, \mathrm{E} 1, \mathrm{~F} 2, \mathrm{G} 5, \mathrm{~K}, \mathrm{~K} 5, \mathrm{M} 1$, and $\mathrm{M} 2$.

This completes the discussion of branches below "Unsaturated Flow in Bedrock."

\section{Saturated Flow in Faults}

"Saturated Flow in Faults" is shown in Paths 3.1 through 3.7 (Tree Segment 3). If faults are highly conductive, they act as channels to convey fluid to depth; if faults are barriers to flow, lateral diversion along dipping strata accumulates perched water against the fault and by the composite-porosity model there is relatively rapid flow to some depth. If the wash is not directly above the repository, as in the case of Drill Hole Wash, additional connections are necessary to convey episodically introduced fluids close enough to the repository for interaction.

Connection from the fault to the repository is either by fractures or by leakage from the fault system to the unsaturated flow field (Sketch F3, p. 29). The composite-porosity model (Peters and Klavetter, 1988) sums the conductivity of pores and fractures with pressure as the independent variable. The effective result is that fracture flow in the microfractures of their model is delayed until all matrix pores are filled. The faults are conduits in either 


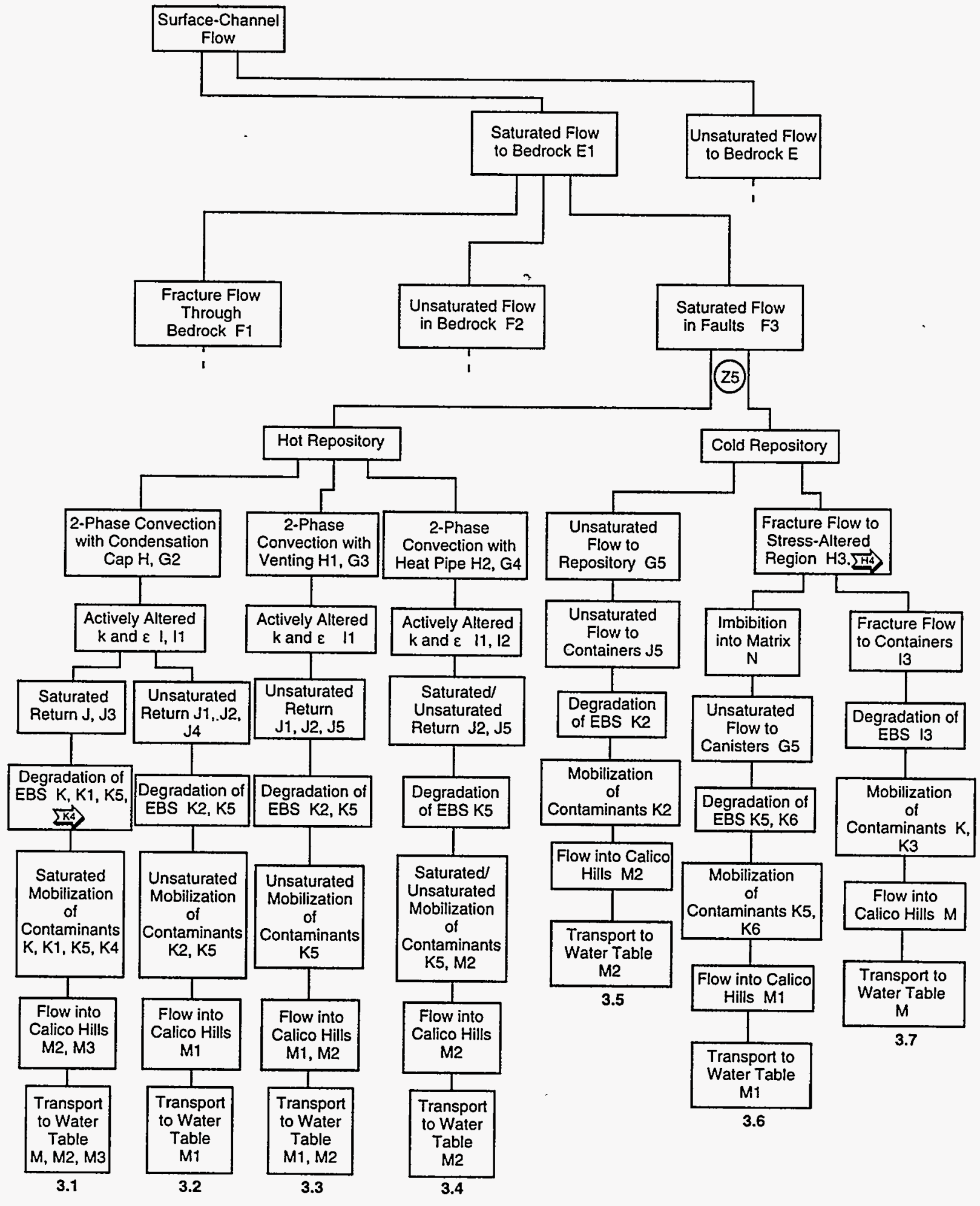

a. Events and their relationships, with cross-references to sketches illustrating each event.

Tree Segment 3. Nominal Flow, Runoff-Producing Events, Runoff to Washes, Washes With Faults, SurfaceChannel Flow, Saturated Flow to Bedrock, Saturated Flow in Faults. 


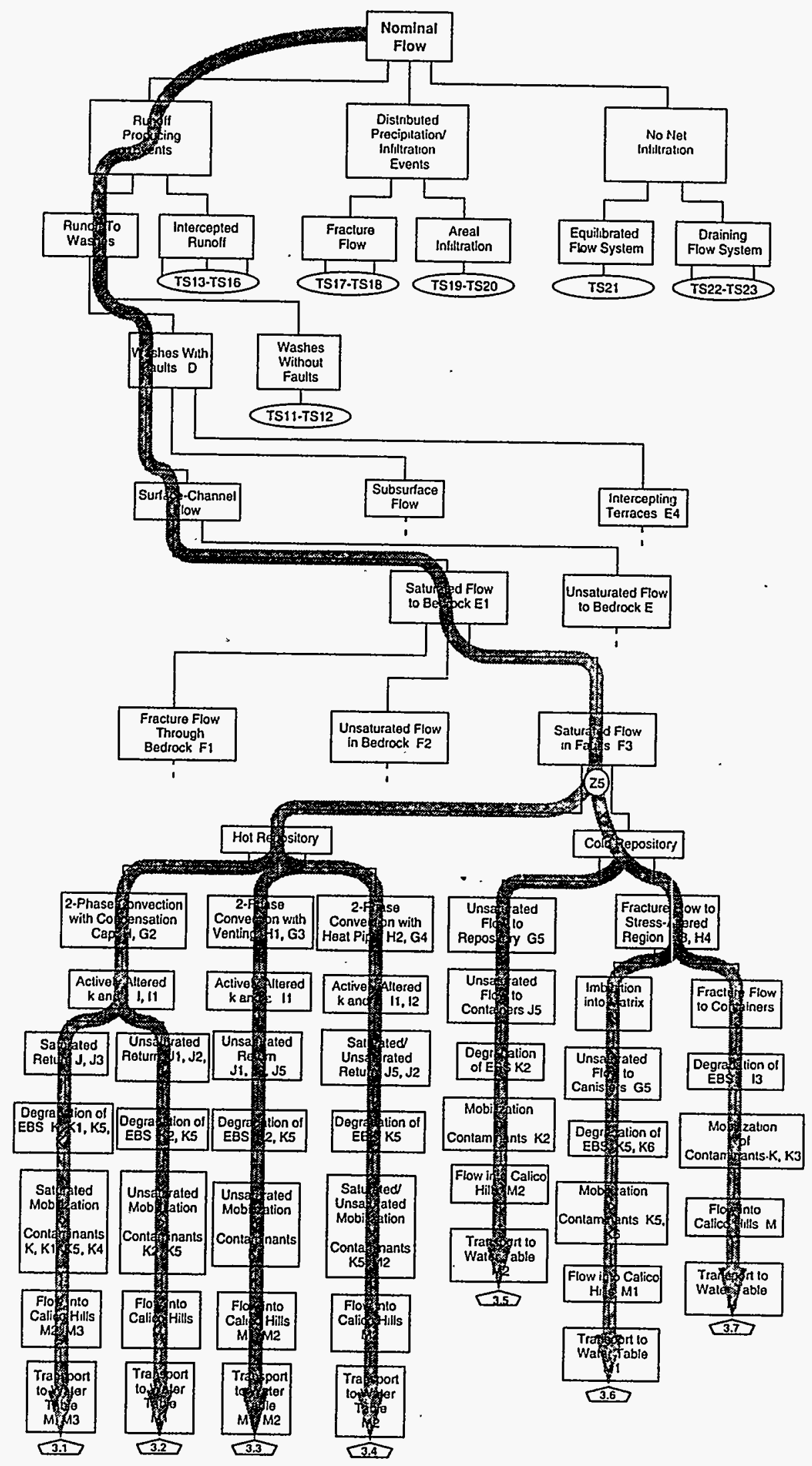

b. Scenario pathways, showing the relationship of the tree segment to the overall event tree for nominal flow. 
sense. What distinguishes them from fractures in bedrock is that we do not expect waste to be put in any fault zone identified in the underground workings. That means, as suggested in Sketch F3, there must be some connection from the faults to the repository. The connections shown in Sketch F3 are fracture connections and unsaturated flow due to leakage from the faults and associated fractures. The two separate cases are treated here in one tree segment. A hot repository or cold repository is distinguished on the usual basis of whether two-phase convection can be driven by repository heat.

Hot Repository, Paths $\widehat{3.1}$ through $\widehat{3.4}$. In a hot repository, flow down the faults (Sketch F3) provides a saturated source of fluids (fractures) and an unsaturated source (leakage to the matrix) for interaction with the repository heat. In effect we are looking at a process that combines fracture flow through bedrock (Sketch F1) and unsaturated flow in bedrock (Sketch F2). As suggested by inclusion of multiple sketches in many steps of the tree branches, the two modes of flow compete for fluid at every step. The two modes have differing time constants for response, and which mode dominates depends on the volume of fluid and rate of influx as well as on the hydraulic properties of the units in the flow path. Flow under these competing, simultaneous processes is a matter being investigated calculationally and experimentally. Paths $\widehat{3.1}, \widehat{3.2}, \widehat{3.3}$, and $\widehat{3.4}$ for the hot repository are similar to those just discussed; how fluids are conveyed to the repository (Sketch F3) has been changed. The scenarios are constructed by merging what was developed earlier. The associated descriptive cartoons are these: .

$$
\begin{aligned}
& \text { C, D, E1, F3, H, G2, I, I1, J, J3, K, K1, K4, K5, M, M2, M3 } \\
& \text { C, D, E1, F3, H, G2, I, I1, J1, J2, J4, K2, K5, M1 } \\
& \text { C, D, E1, F3, H1, G3, I1, J1, J2, J5, K2, K5, M1, M2 } \\
& \text { C, D, E1, F3, H2, G4, I1, I2, J2, J5, K5, M2 }
\end{aligned}
$$

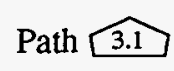

Path 3.2

Path 3.3

Path $\widehat{3.4}$

Cold Repository, Paths 3.5 through 3.7 . We have assumed that fault flow is significant if fluids can be conducted laterally far enough to interact with the repository (Sketches H3 and H4). Connecting fractures are necessary to get fluid close to the repository-presumably to the stress-altered region. This assumption is built on current preliminary calculations of the temperature field. If our interpretation of these requirements is erroneous, we will need to consider a volume larger than the stress-altered zone.

The case for the cold repository has been expanded somewhat in order to suggest how such an expansion might be developed for modeling. There are three paths expanded below a cold repository. First (Path $\widehat{3.5}$ ), an unsaturated plume may form from flow in faults before reaching wastes. Second (Path $\widehat{3.6}$ ), the plume may form after fracture flow to the stress-altered region. Third (Path 3.7 ), fracture flow may reach waste containers.

Path $\widehat{3.5}$ describes the case of unsaturated flow to the repository-unsaturated flow that originates from imbibition or leakage from the fault zone and local fractures. This unsaturated flow arrives at the repository (Sketch G5) and, navigating through the fractures of the stress-altered zone, reaches the waste containers (Sketch J5). Degradation of the EBS accelerates upon arrival of this unsaturated flow (Sketch K2) and mobilization of 


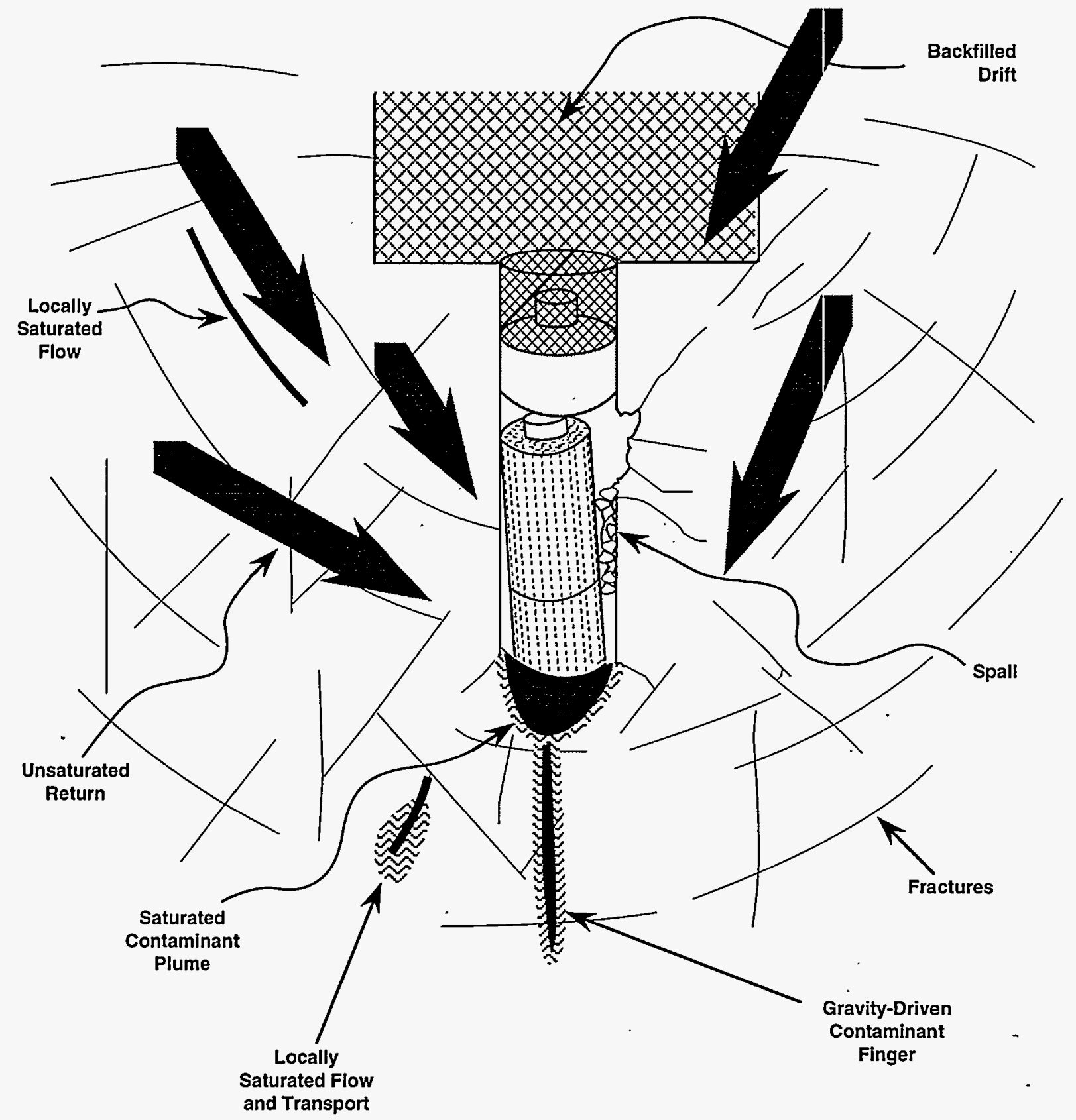

Sketch K4. Degradation of the EBS and mobilization of contaminants in the presence of a saturated flux (composite model) that reaches the waste container and produces a saturated contaminant flow plume. 


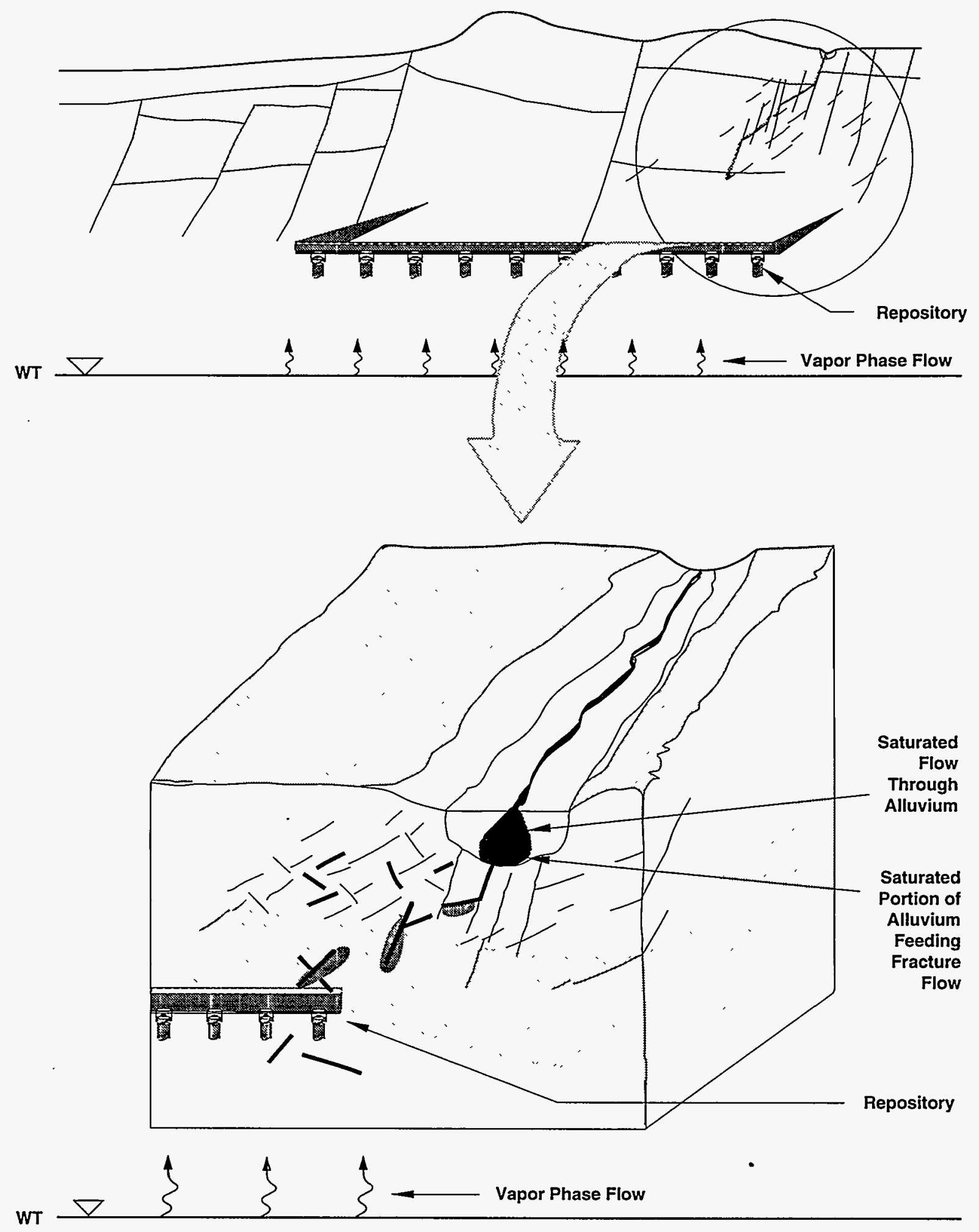

Sketch H4. Migration of fluids laterally to interact with the repository. 
contaminants begins (Sketch K2). Flow into the Calico Hills units and on to the water table is in an unsaturated, locally saturated flow field (Sketch M2). Path $\widehat{3.5}$ encompasses C, D, E1, F3, G5, J5, K2, and M2.

For Paths 3.6 and 3.7 , there is fracture flow from the fault zone to the stress-altered region (Sketch H4). In Path $\widehat{3.6}$, flow is imbibed by the matrix in the stress-altered region so fracture flow is transformed to unsaturated/saturated flow (Sketch $\mathrm{N}$ ). Sketch $\mathrm{J} 5$ depicts this transformed unsaturated flow reaching the waste container, augmenting its degradation, and mobilizing the movement of contaminants (Sketch K6). Flow to the Calico Hills units and down to the water table then proceeds in an unsaturated flow system (Sketch M1). The scenario described by Sketches C, D, E1, F3, H3, H4, N, G5, K5, K6, and M1 for fault flow imbibed by the matrix is shown in Path 3.6 .

In Path 3.7, fracture flow continues to the waste container (Sketch 13), so a locally saturated flow system exists to provide fluids. In reality, we expect a combination of this fracture flow branch and the imbibition branch, but this discussion points separately to ideas that should be included in any modeling. Degradation of the EBS is indicated by stand-in figures, showing fluid arrival and contaminant mobilization (Sketch $\mathrm{K} 3$ ). Without more detail on radiochemical interactions, we are unable to sketch more specifically. The waste container is further degraded in a saturated environment (Sketch $\mathrm{K} 1$ ) and contaminants are mobilized into both fracture flow and unsaturated leakage (fingers). Flow into the Calico Hills units and to the water table could occur by either unsaturated flow or fracture flow or both (Sketch M). With transport to the water table we have constructed the scenario described by Sketches $\mathrm{C}, \mathrm{D}, \mathrm{E} 1, \mathrm{~F} 3, \mathrm{H} 3, \mathrm{H} 4, \mathrm{I} 3, \mathrm{~K}, \mathrm{~K} 3$, and $\mathrm{M}$ for fracture flow from faults to the waste containers.

This concludes discussion of saturated flow to bedrock from a surface channel in washes with faults.

\section{Unsaturated Flow to Bedrock, Paths 4.1 Through 6.6}

The previous section discussed surface flow in washes with faults that reaches bedrock by means of saturated flow in the alluvium (Sketch E1, p. 25). There is an alternative way by which surface flow from the channel in the wash may reach bedrock, namely as unsaturated flow in the alluvium beneath the channel (Sketch E, p. 24). Tree Segments 4, 5, and 6 are parallel to Tree Segments 1,2, and 3; they also concern fracture flow and unsaturated flow in bedrock and saturated flow in faults. Only the source is changed; in the case of Sketch E, the source is an unsaturated but not drained alluvium below the wash channel which is draining to the bedrock. Such a source is distinguished from a saturated alluvium, for our purposes, because there are different implications for the duration and frequency of runoff-producing storms. That means that volume, rate, and probability of occurrence are also different. When a distribution function is available to describe volume, rate, and frequency of the storms, we can lump the two (saturated flow to bedrock and unsaturated flow to bedrock) into a single model. The scenarios in these tree segments are similar to those already developed, so the scenarios are simply listed. Sketches E, F (p. 30), F4, and F5 replace Sketches E1, F1, F2, and F3 respectively: 


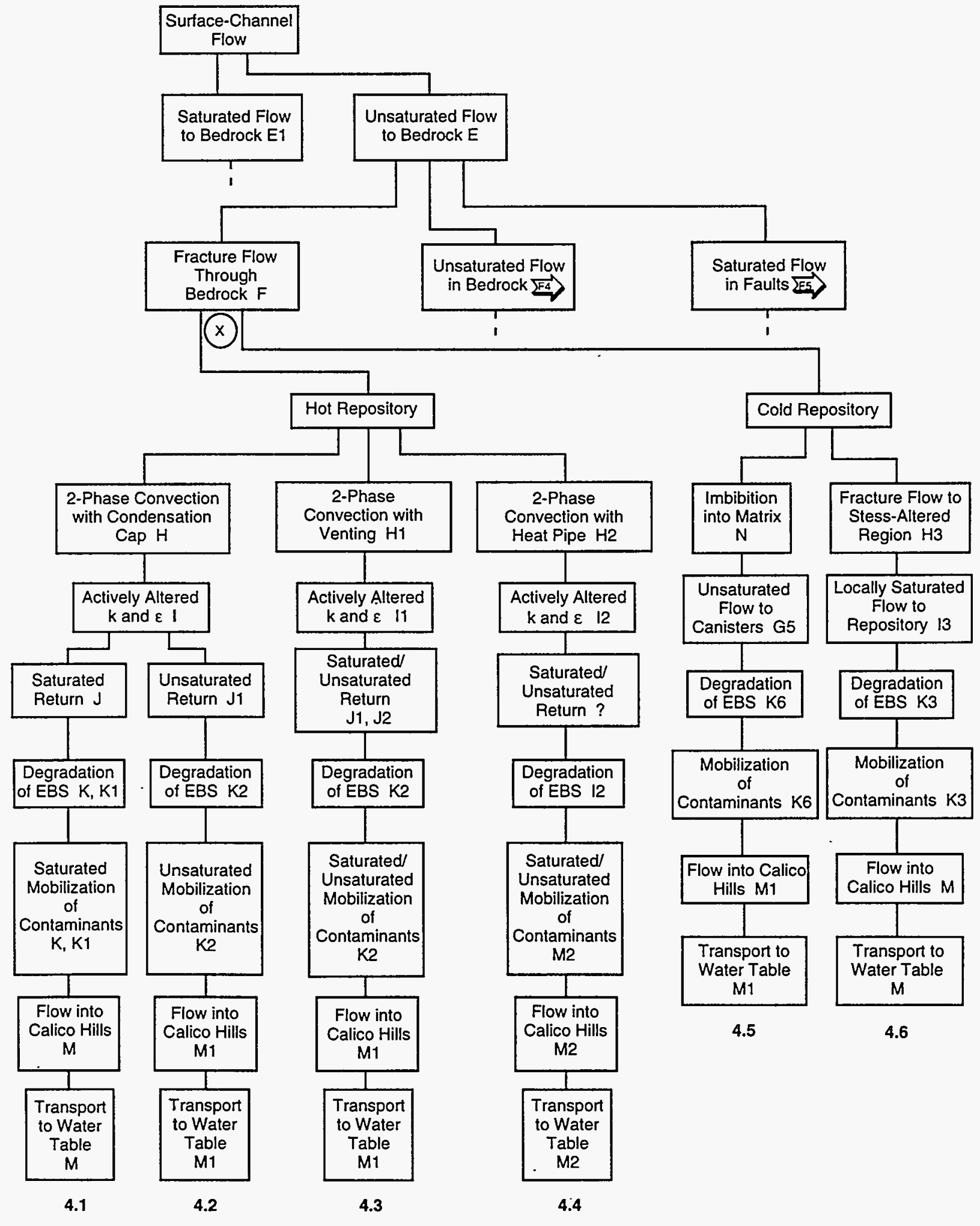

a. Events and their relationships, with cross-references to sketches illustrating each event.

Tree Segment 4. Nominal Flow, Runoff-Producing Events, Runoff to Washes, Washes With Faults, SurfaceChannel Flow, Unsaturated Flow to Bedrock, Fracture Flow Through Bedrock. 


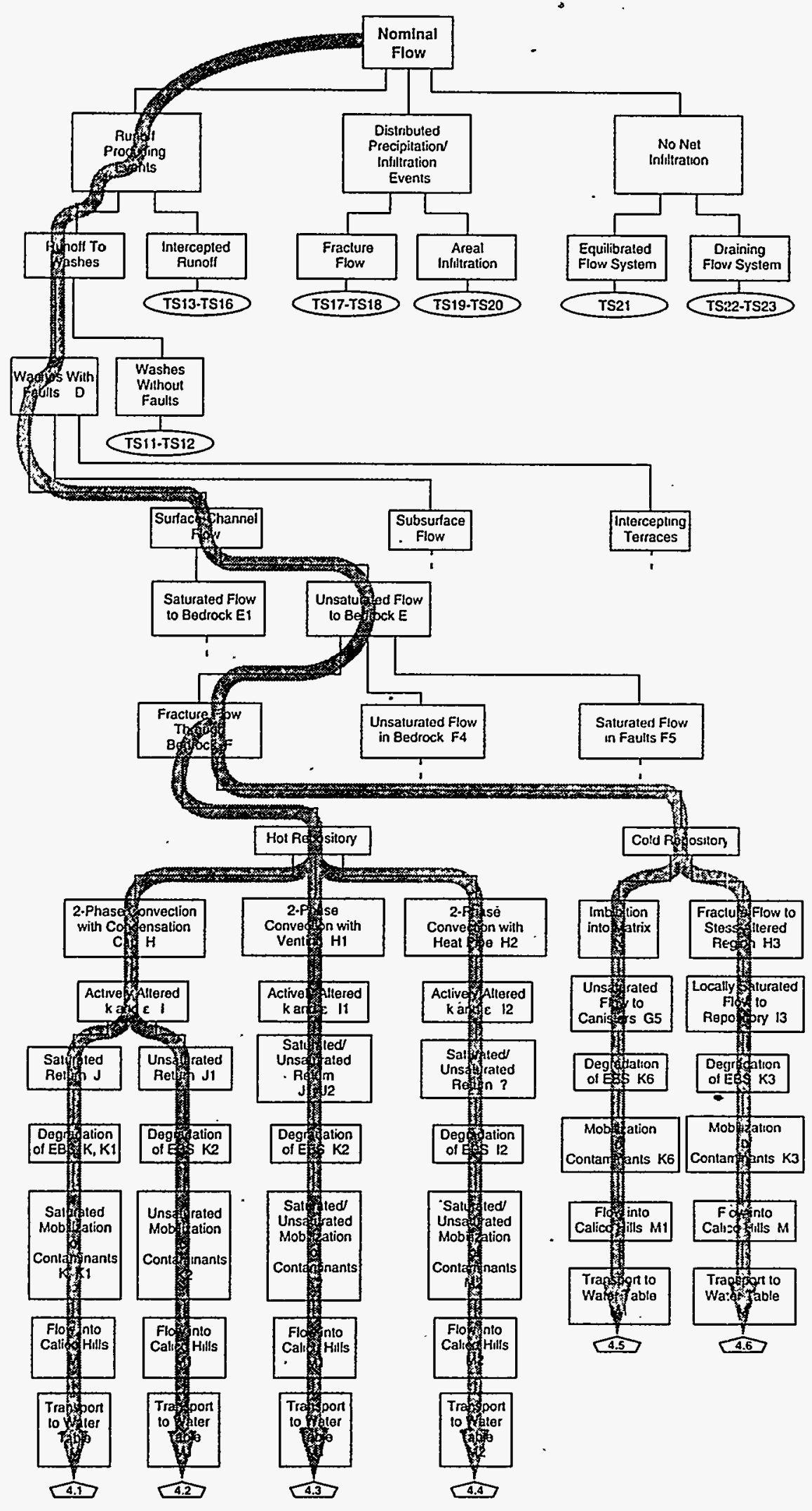

b. Scenario pathways, showing the relationship of the tree segment to the overall event tree for nominal flow. 


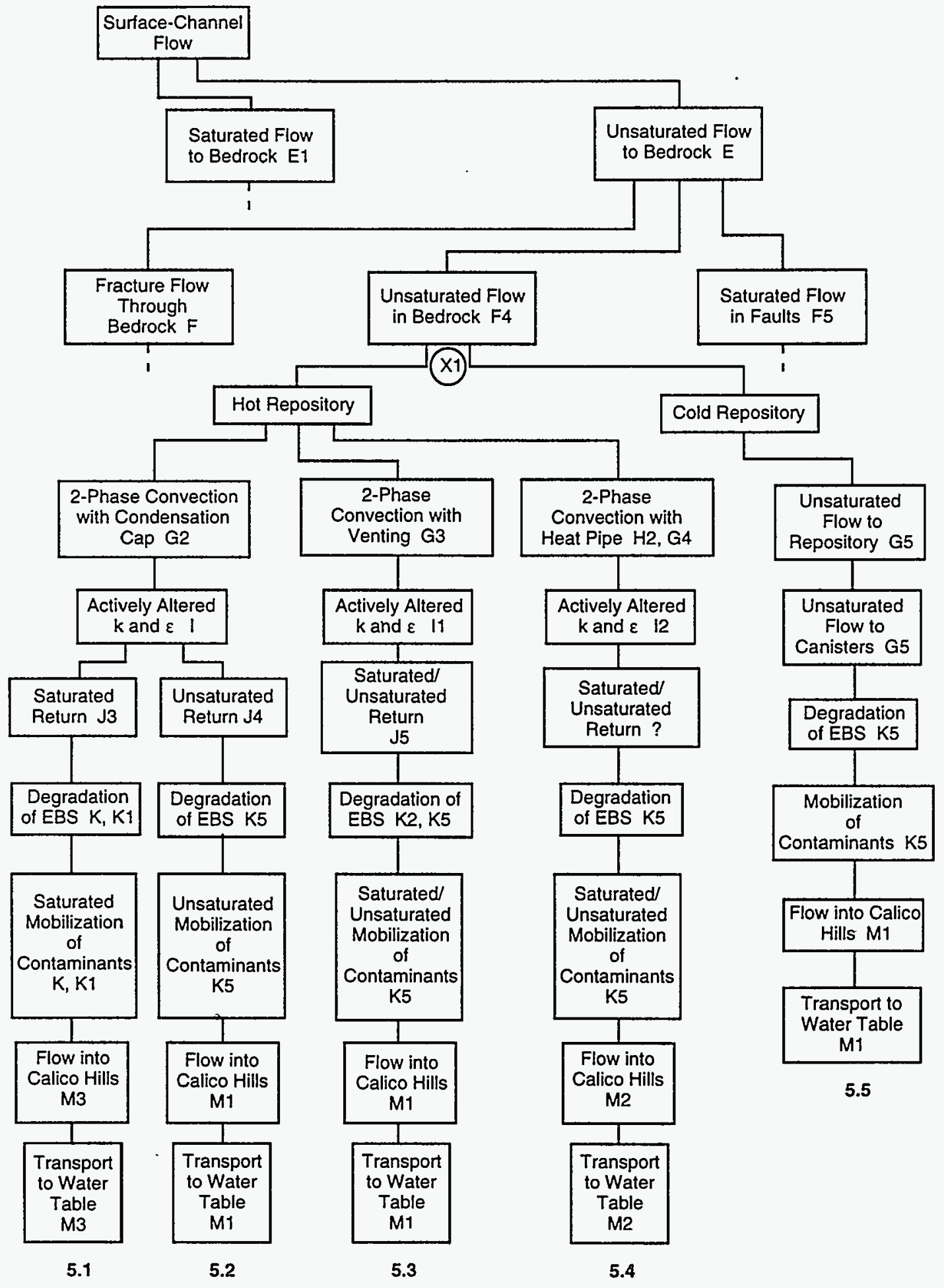

a. Events and their relationships, with cross-references to sketches illustrating each event.

Tree Segment 5. Nominal Flow, Runoff-Producing Events, Runoff to Washes, Washes With Faults, SurfaceChannel Flow, Unsaturated Flow to Bedrock, Unsaturated Flow in Bedrock. 

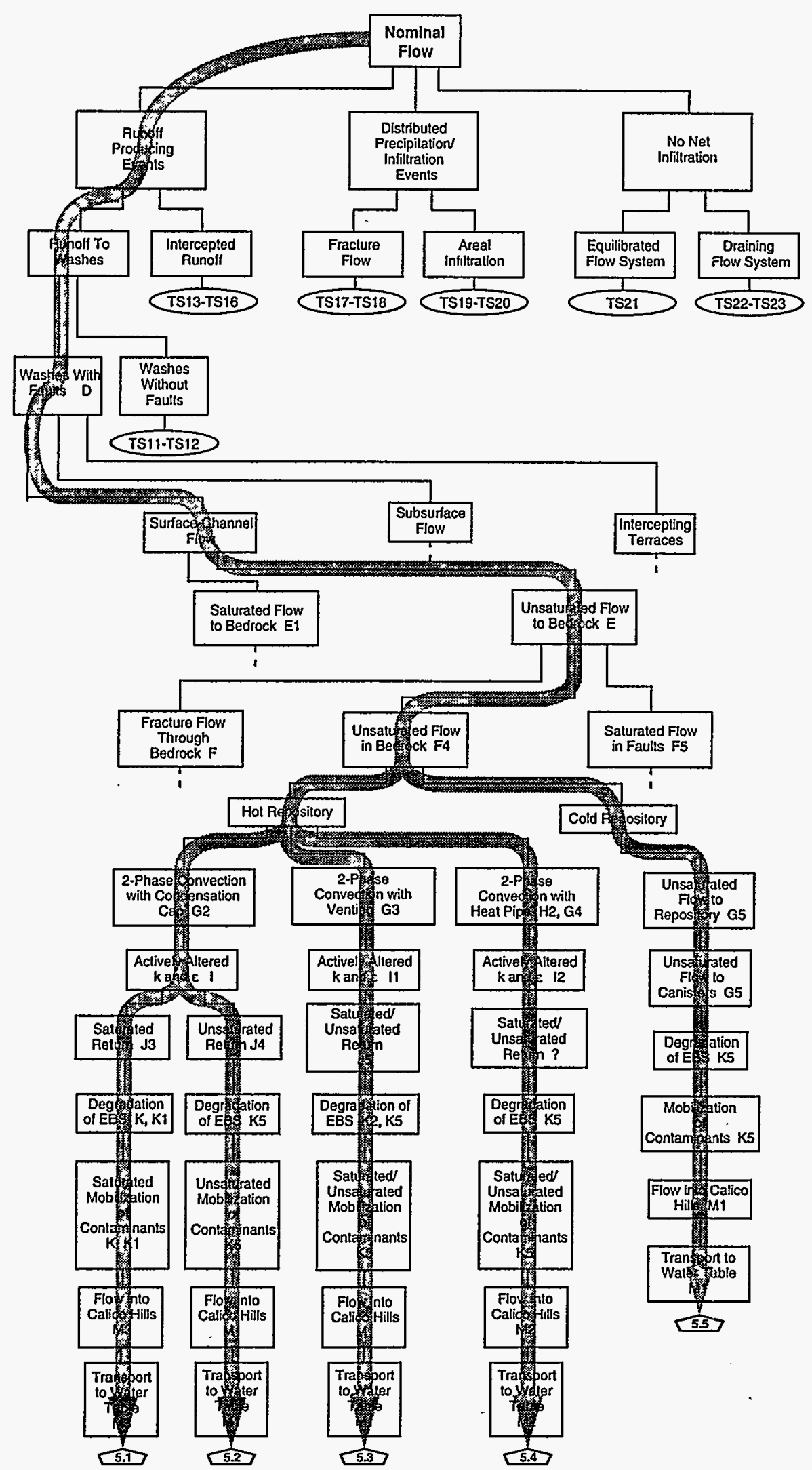

b. Scenario pathways, showing the relationship of the tree segment to the overall event tree for nominal flow. 


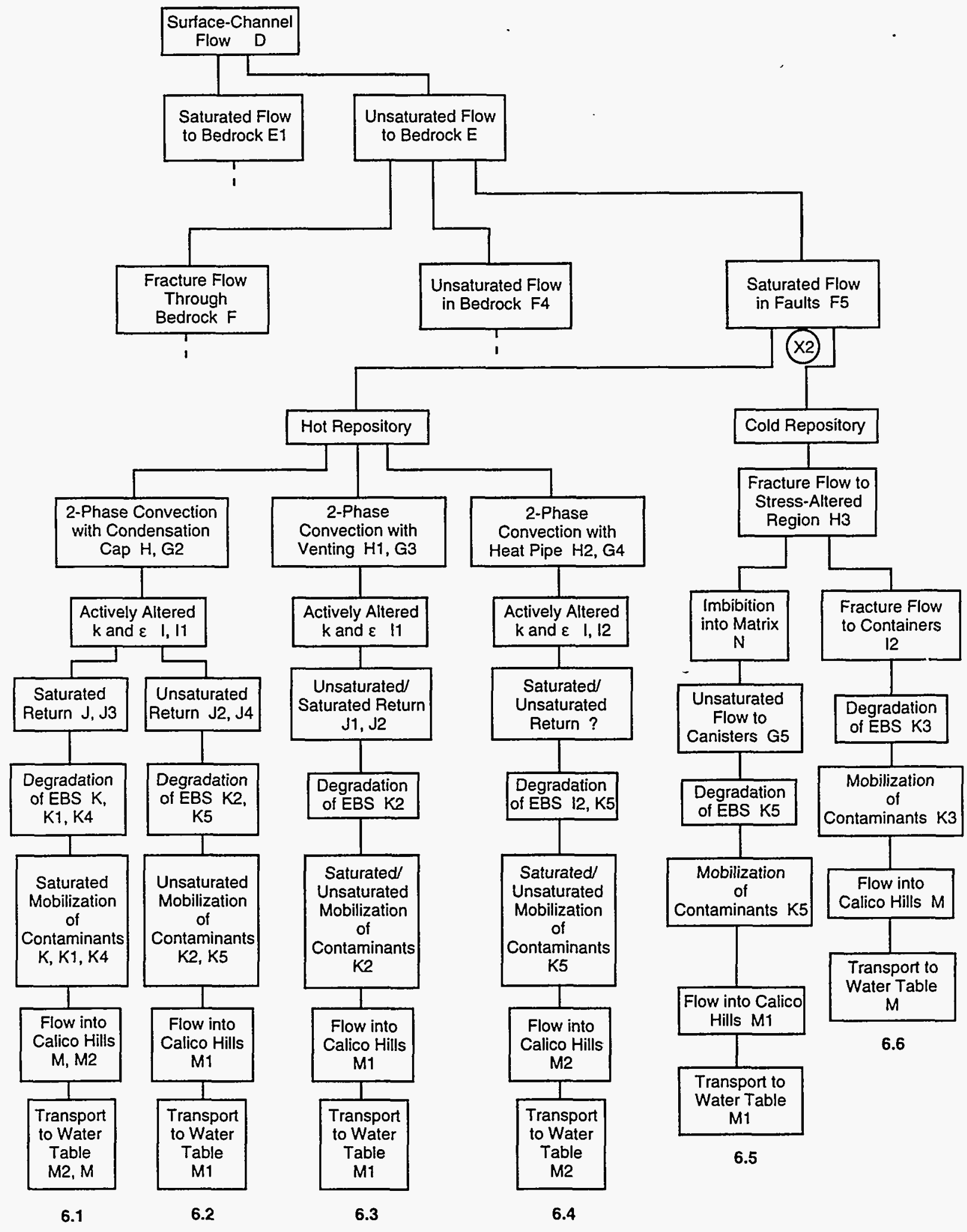

a. Events and their relationships, with cross-references to sketches illustrating each event.

Tree Segment 6. Nominal Flow, Runoff-Producing Events, Runoff to Washes, Washes With Faults, SurfaceChannel Flow, Unsaturated Flow to Bedrock, Saturated Flow in Faults. 


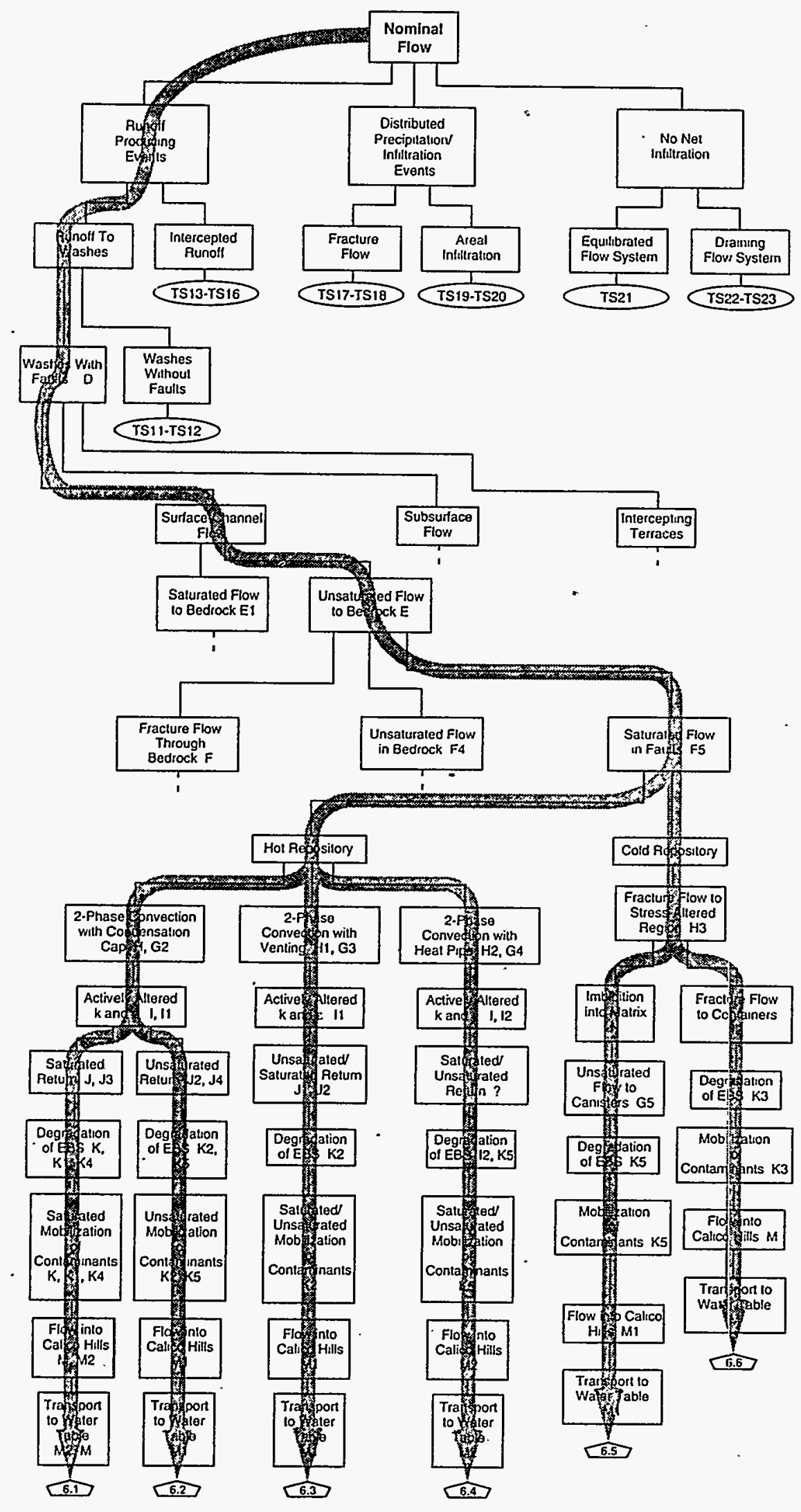

b. Scenario pathways, showing the relationship of the tree segment to the overall event tree for nominal flow. 


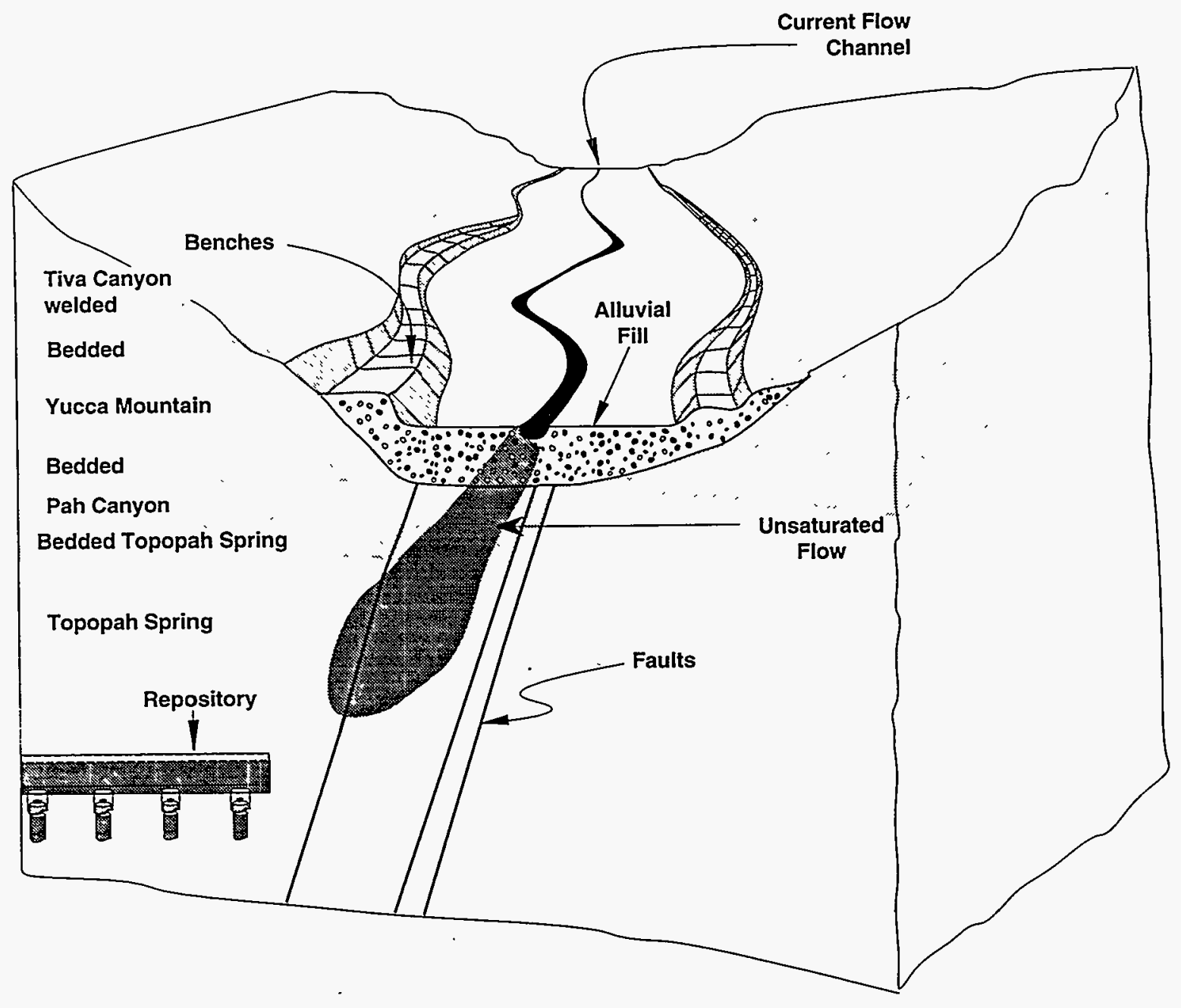

Sketch F4. Unsaturated flow in alluvium followed by unsaturated flow in bedrock. 


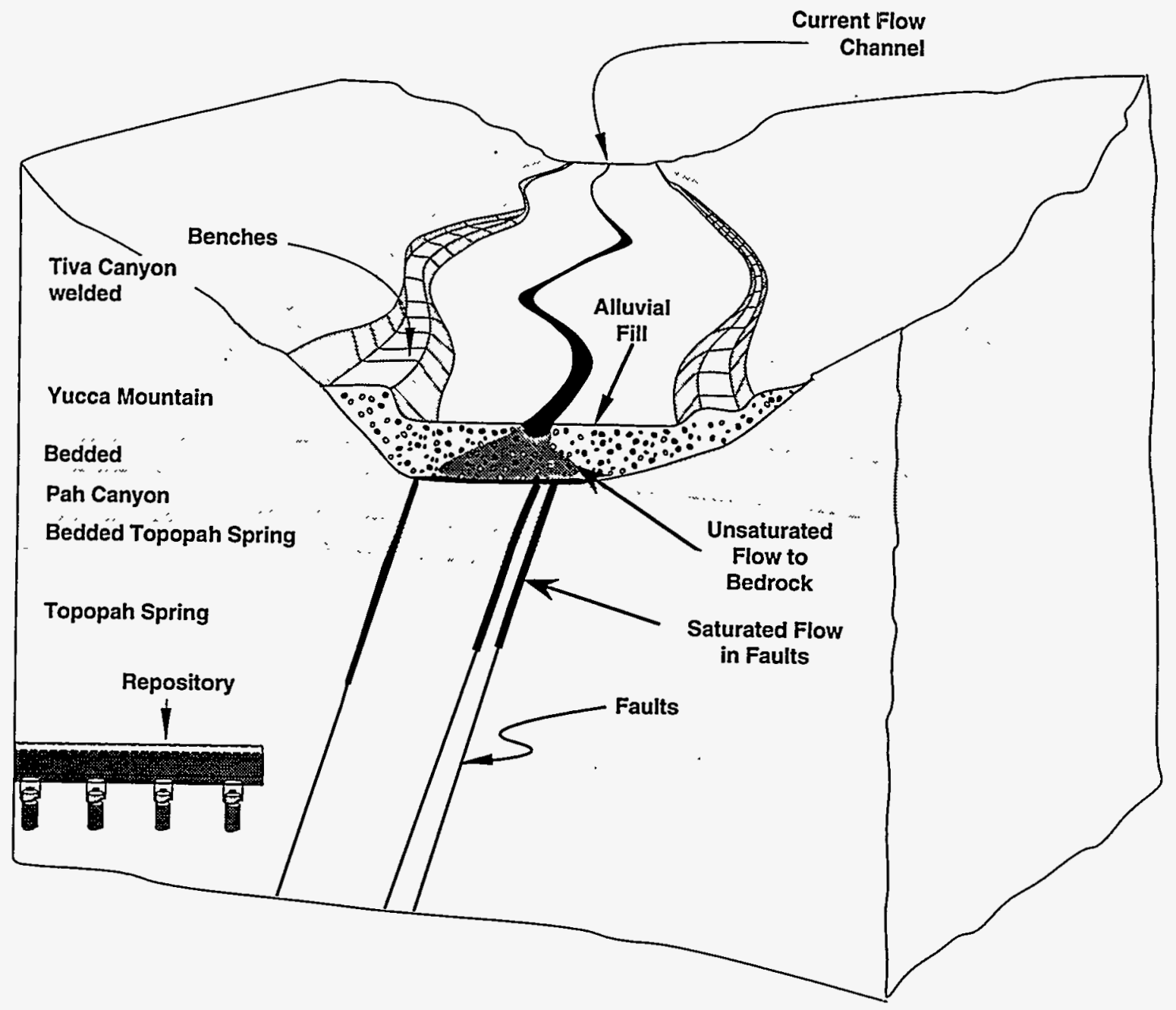

Sketch F5. Unsaturated flow in alluvium followed by saturated flow in faults. 
C, D, E, F, H, I, J, K, K1, M

C, D, E, F, H, I, J1, K2, M1

C, D, E, F, H1, I1, J1, J2, K2, M1

C, D, E, F, H2, I2, ?, M2

C, D, E, F, N, G5, K6, M1

C, D, E, F, H3, I3, K3, M

C, D, E, F4, G2, I, J3, K, K1, M3

C, D, E, F4, G2, I, J4, K5, M1

C, D, E, F4, G3, I1, J5, K2, K5, M1

C, D, E, F4, H2, G4, I2, ?, K5, M2

C, D, E, F4, G5, K5, M1

C, D, E, F5, H, G2, I, I1, J, J3, K, K1, K4, M, M2

C, D, E, F5, H, G2, I, I1, J2, J4, K2, K5, M1

C, D, E, F5, H1, G3, I1, J1, J2, K2, M1

C, D, E, F5, H2, G4, I1, I2, ?, K5, M2

C, D, E, F5, H3, N, G5, K5, M1

C, D, E, F5, H3, I2, K3, M
Path 4.1

Path 4.2

Path 4.3

Path 4.4

Path 4.5

Path 4.6

Path 5.1

Path 5.2

Path 5.3

Path 5.4

Path 5.5

Path 6.1

Path 6.2

Path 6.3

Path 6.4

Path 6.5

Path 6.6

This completes the discussion of scenarios beginning with surface flow in washes with faults.

\subsubsection{Subsurface Flow, Tree Segment 7}

We now discuss scenarios beginning with subsurface flow in washes with faults for two cases, flow in the alluvium and flow along the bedrock-alluvium contact.

Flow in the alluvium (Sketch E2) is a special case of Sketch E, "Unsaturated Flow to Bedrock," for which scenarios have already been discussed. The fact that the unsaturated-flow tube is locally unconnected with the flow channel depends on the volume of runoff and local inhomogeneities of the alluvium. In general, the unsaturatedflow tube probably extends to bedrock in a few places rather than everywhere, except when the flow channel runs water for some substantial period. The behavior of flow in alluvium has enough peculiarities that we call attention to it for modeling so that it will be included in calculations of unsaturated flow to bedrock. Scenarios constructed in the previous section (Sketches F, F4, and F5) will be presumed to include flow in the alluvium.

Flow at the bedrock-alluvium contact (Tree Segment 7) differs somewhat from the vertical flow discussed earlier. Fluid could reach the contact as runoff that penetrates alluvium along the edge of the wash and feeds this contact (Sketch E3). Fluid also can flow along this contact once it reaches it from the flow tube beneath the flow channel-discussed earlier. Flow along the contact is likely to consist of both contributions. Once flow is at the bedrock-alluvium contact, the processes for movement to the repository are similar to those below Sketches $\mathrm{E}$ 


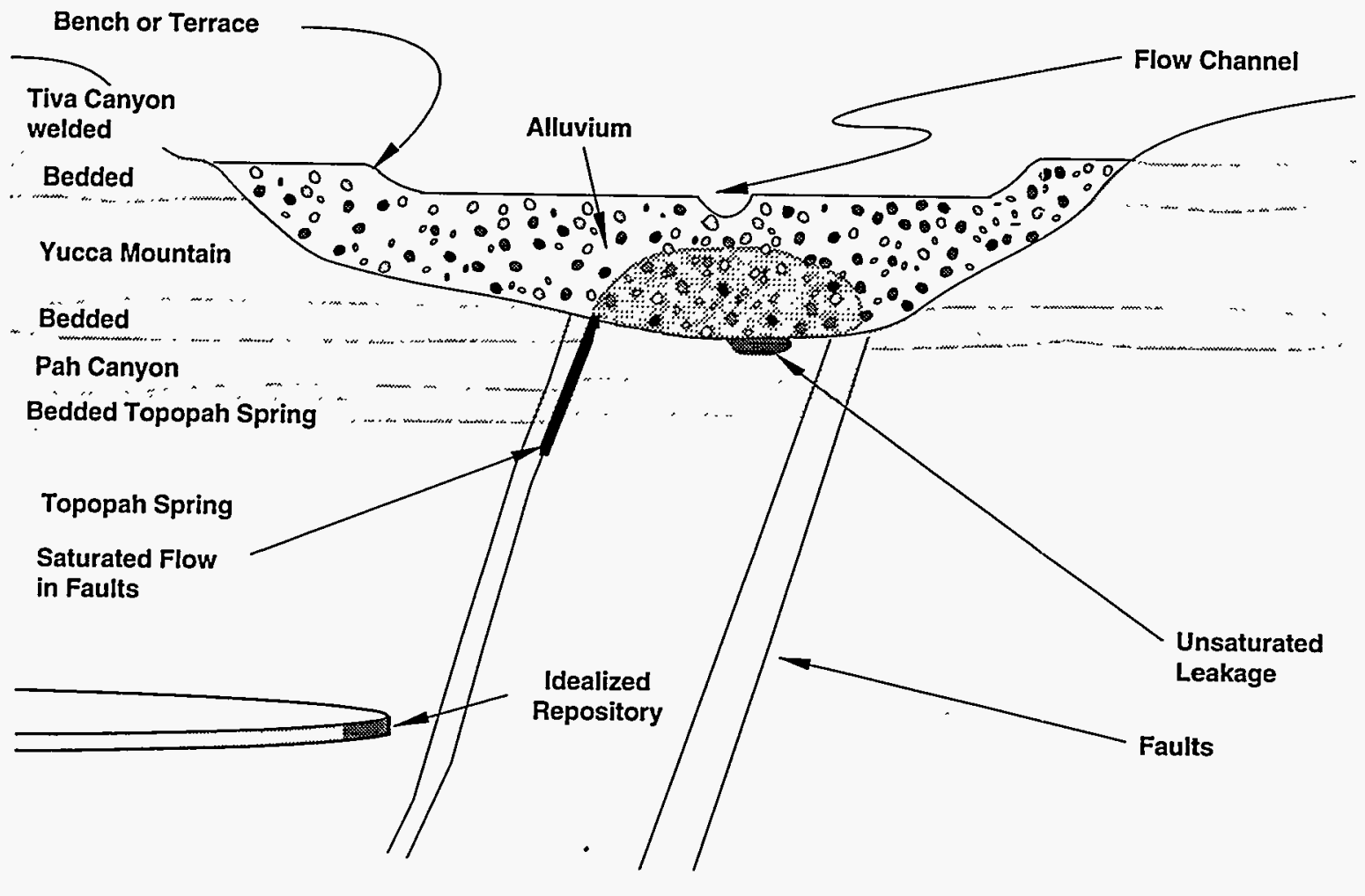

Sketch E2. Small-scale diagrammatic cross section of a wash with faults, showing a zone of unsaturated flow that is locally unconnected with the flow channel. 


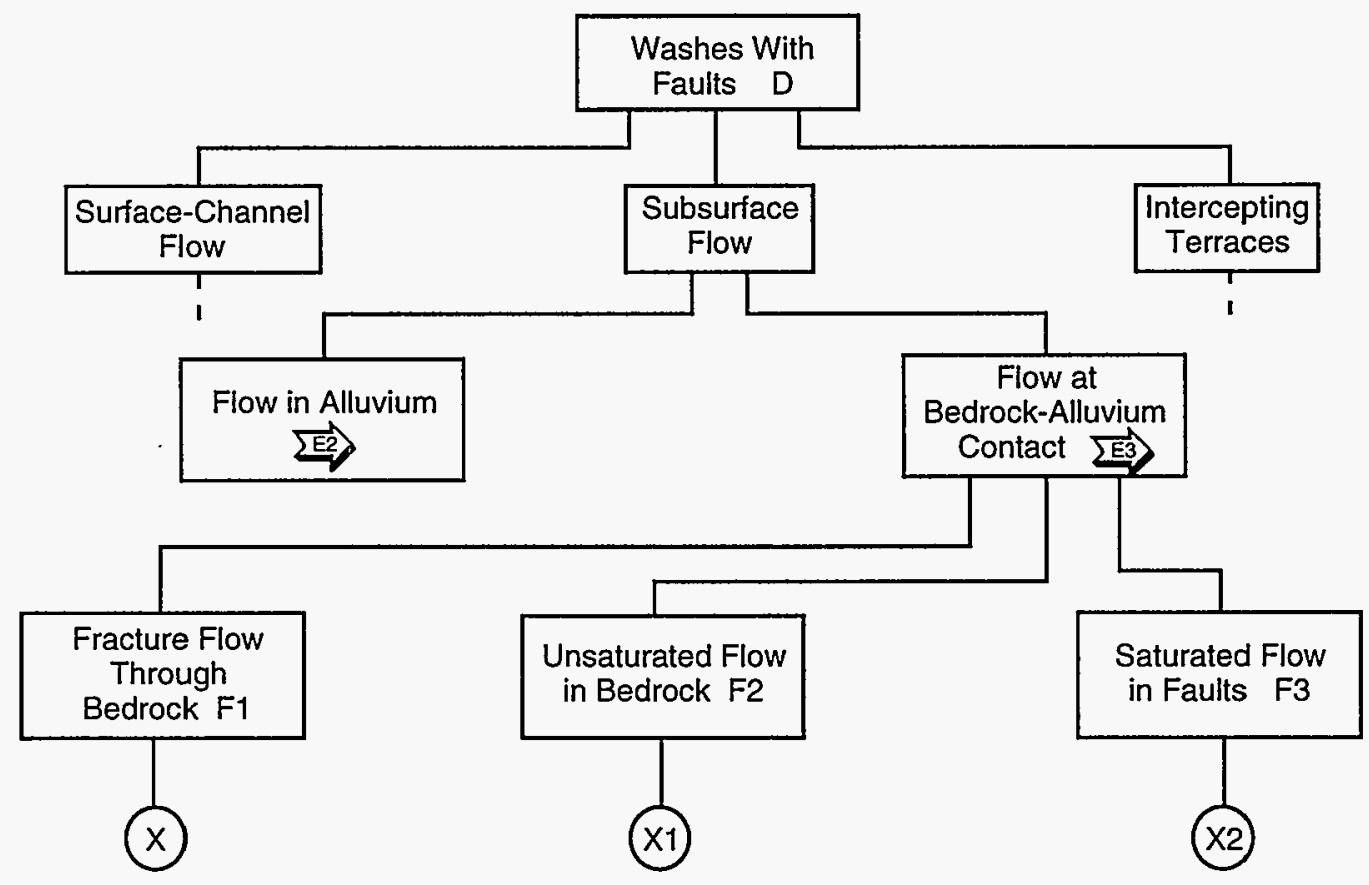

a. Events and their relationships, with cross-references to sketches illustrating each event.

Tree Segment 7. Nominal Flow, Runoff-Producing Events, Runoff to Washes, Washes With Faults, Subsurface Flow, Flow at Bedrock-Alluvium Contact. 


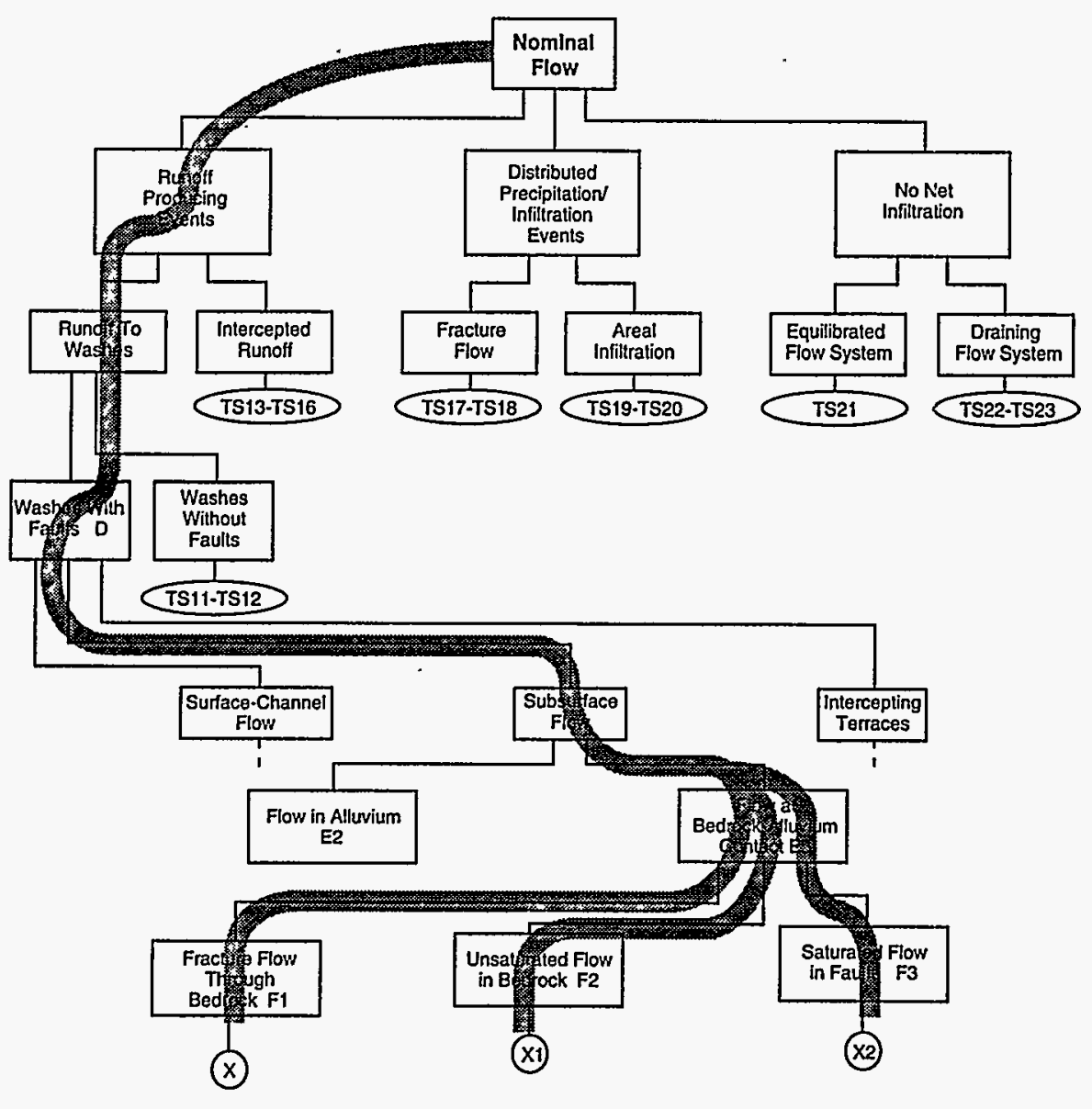

b. Scenario pathways, showing the relationship of the tree segment to the overall event tree for nominal flow. 


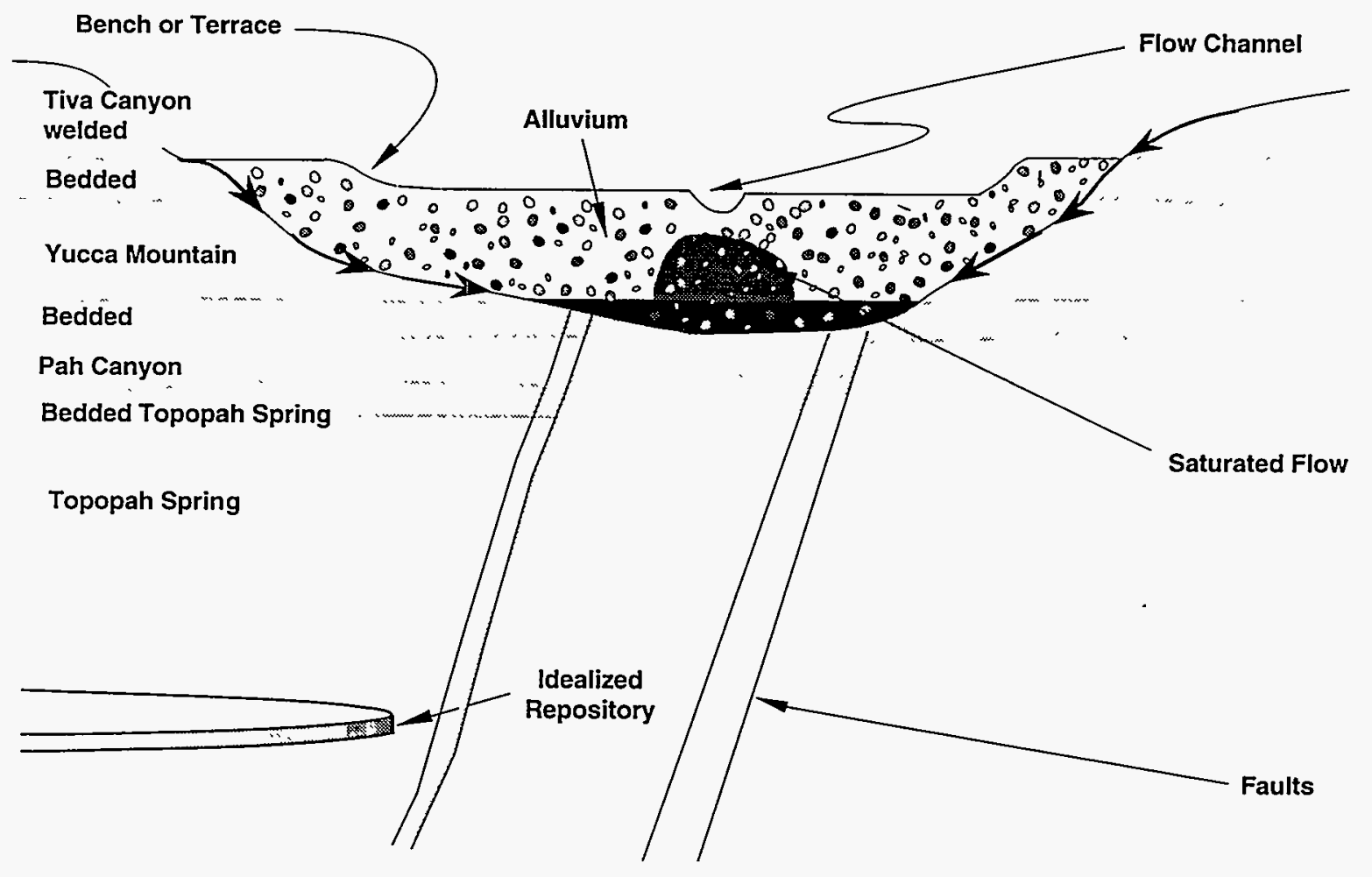

Sketch E3. Small-scale diagrammatic cross section of a wash with faults, showing a zone of saturated flow that is locally unconnected with the flow channel. 
and E1, namely "Fracture Flow Through Bedrock, F1," "Unsaturated Flow in Bedrock, F2," and "Saturated Flow in Faults, F3." Both Sketches E and E1 are relevant because the source of fluid is ephemeral and flow through the alluvium changes between saturated flow and unsaturated flow as fluid leaks into bedrock or leaves the area down wash. The scenarios are then the list just given, with E3 substituted for E, F1 for F, F2 for F4, and F3 for F5.

\subsubsection{Interception by Terraces, Tree Segments 8 through 10}

In washes with faults there is another possibility for interception of runoff, namely interception by benches or terraces above the channel (Tree Segments 8,9 , and 10 ). Such a flat area was probably part of a wash bottom before down-cutting produced the current flow channel. It is typically discontinuous and situated against the slope. Because it is relatively flat, it intercepts runoff (Sketch E4, p. 98) and directs it to a local flow system. The local flow system provides these fluids to the bedrock/alluvium interface either as saturated or unsaturated flow. The intercepting bench acts as a buffer for the fluid source to bedrock. Essentially, the behavior of such a buffer is a special case in each of the cases E, E1, E2, and E3 considered earlier. As long as the runoff is accounted for in toto there seems to be no point in discussing this mode of infiltration separately. Because the sequence of events below "Intercepting Terraces," EA, and "Saturated Flow to Bedrock," E1, differs slightly from the sequence discussed above, tree segments are included here to support any future work, and the paths are listed below:

C, D, EA, E1, F1, H, I, J, K, K1, M

C, D, E4, E1, F1, H, I, J1, K2, M1

C, D, E4, E1, F1, H1, I1, J2, J 5, K2, M1

C, D, E4, E1, F1, H2, I2, ?, K2, M2

C, D, EA, E1, F1, N, G5, K6, M1

C, D, E4, E1, F1, H3, I3, K3, M

C, D, E4, E1, F2, G2, I, J3, K, K1, M3

C, D, E4, E1, F2, G2, I, J4, K5, M1

C, D, E4, E1, F2, G3, I1, J5, K2, K5, M1

C, D, E4, E1, F2, H2, G4, I2, ?, K5, M2

C, D, E4, E1, F2, G5, K5, M1

C, D, E4, E1, F3, H, G2, I, I1, J, J3, K, K1, K4, M, M2

C, D, E4, E1, F3, H, G2, I, I1, J2, J4, K2, K5, M1

C, D, E4, E1, F3, H1, G3, I1, J1, J2, K2, M1

C, D, E4, E1, F3, H2, G4, I, I2, ?, K5, M2

C, D, E4, E1, F3, H3, N, G5, K5, M1 .

C, D, E4, E1, F3, H3, 13, K3, M
Path $\widehat{8.1}$
Path $\widehat{8.2}$
Path $\widehat{8.3}$
Path $\widehat{8.4}$
Path $\widehat{8.5}$
Path 8.6
Path 9.1
Path $\widehat{9.2}$
Path $\widehat{9.3}$
Path 9.4
Path 9.5
Path 10.1
Path 10.2
Path 10.3
Path $\widehat{10.4}$
Path $\widehat{10.5}$
Path 10.0 


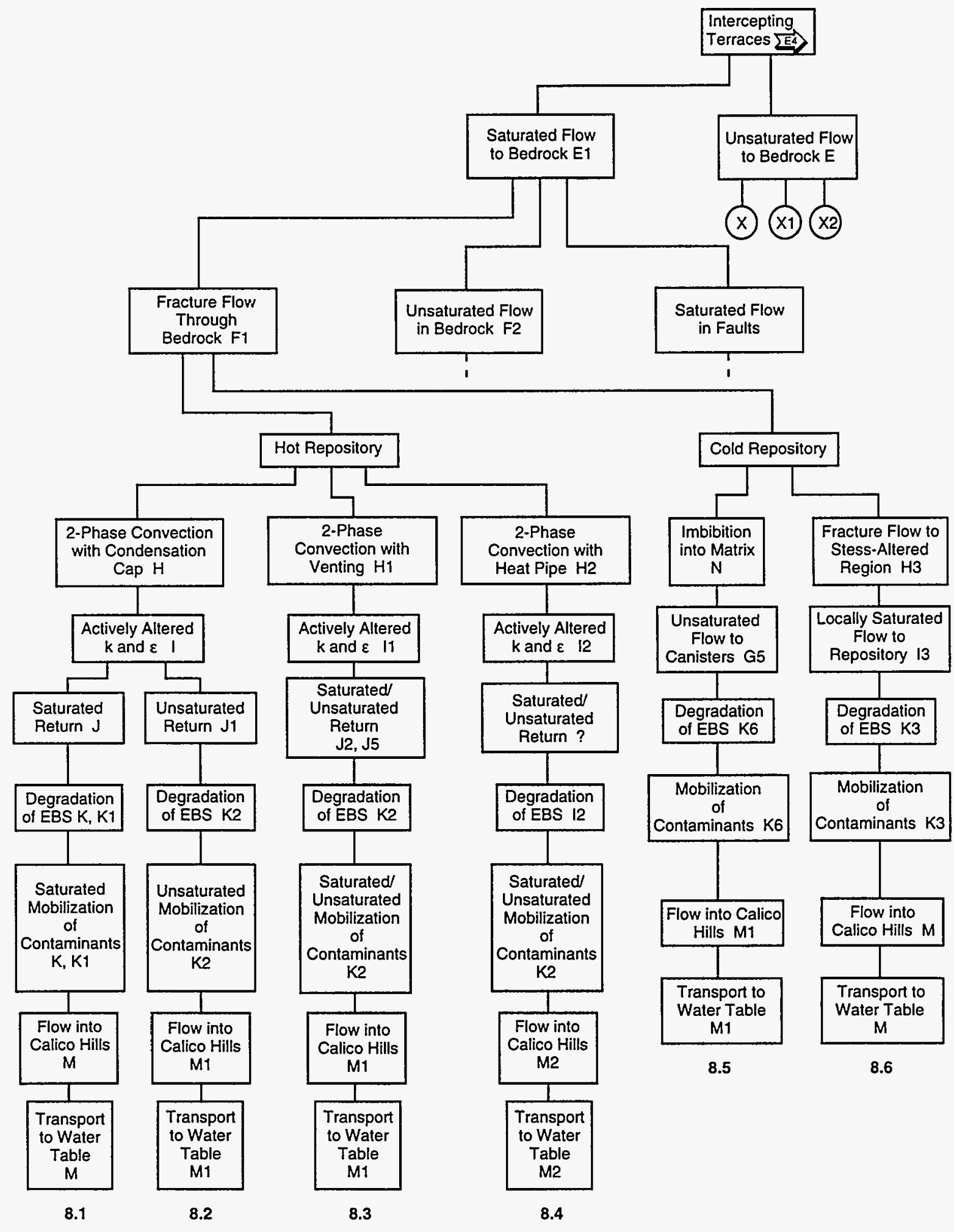

a. Events and their relationships, with cross-references to sketches illustrating each event.

Tree Segment 8. Nominal Flow, Runoff-Producing Events, Runoff to Washes, Washes With Faults, Intercepting Terraces, Saturated Flow to Bedrock, Fracture Flow Through Bedrock. 


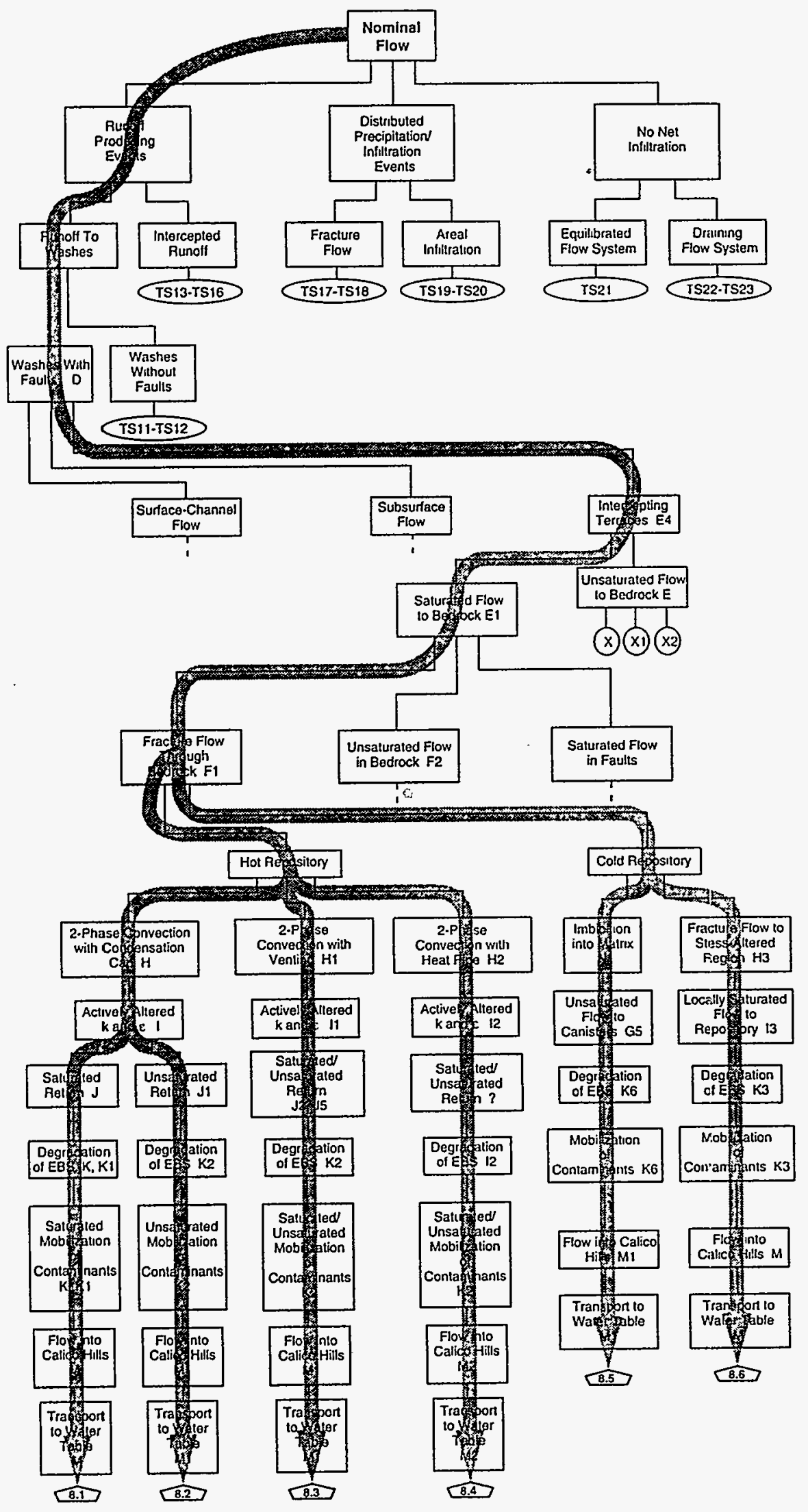

b. Scenario pathways, showing the relationship of the tree segment to the overall event tree for nominal flow. 


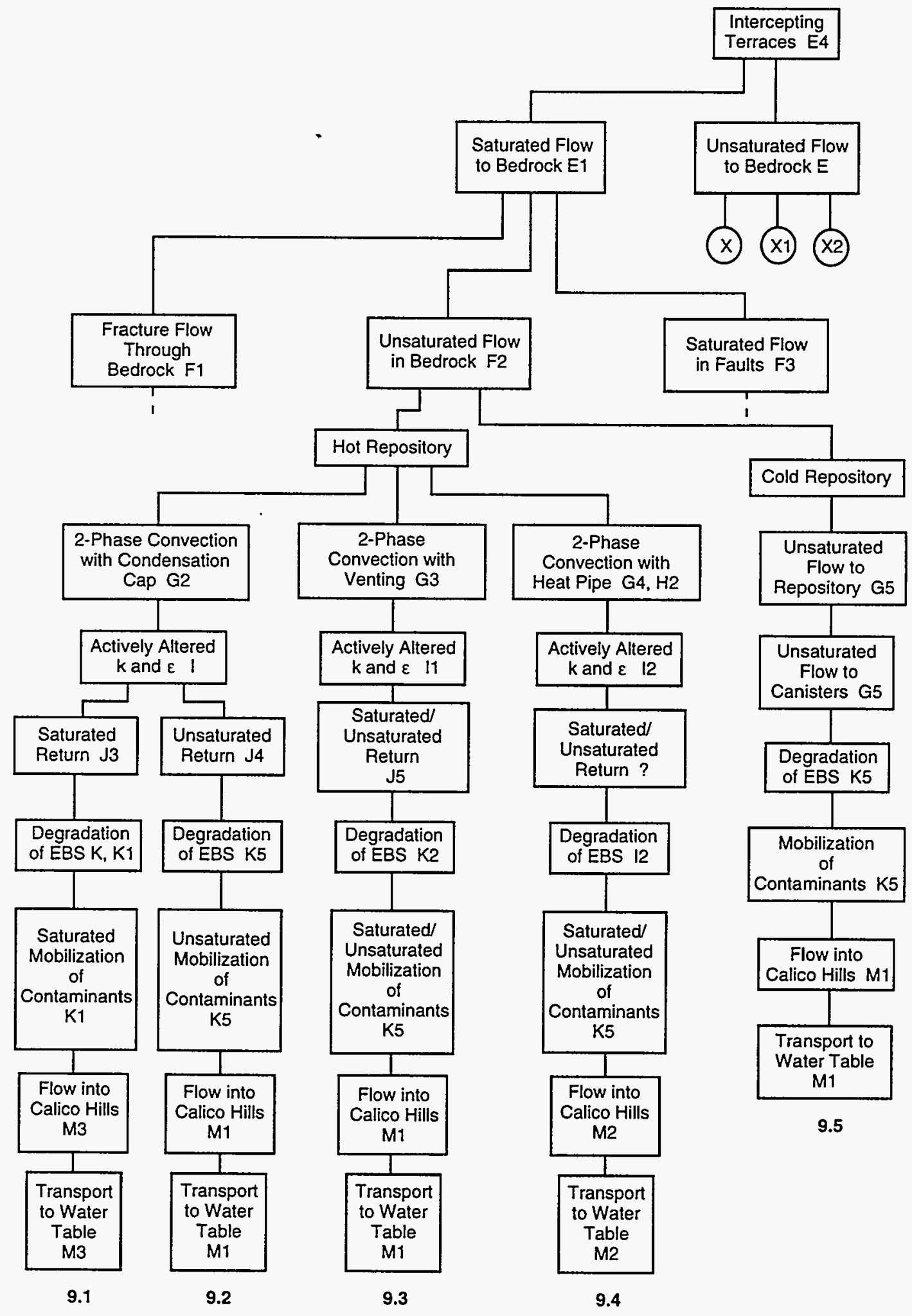

a. Events and their relationships, with cross-references to sketches illustrating each event.

Tree Segment 9. Nominal Flow, Runoff-Producing Events, Runoff to Washes, Washes With Faults, Intercepting Terraces, Saturated Flow to Bedrock, Unsaturated Flow in Bedrock. 


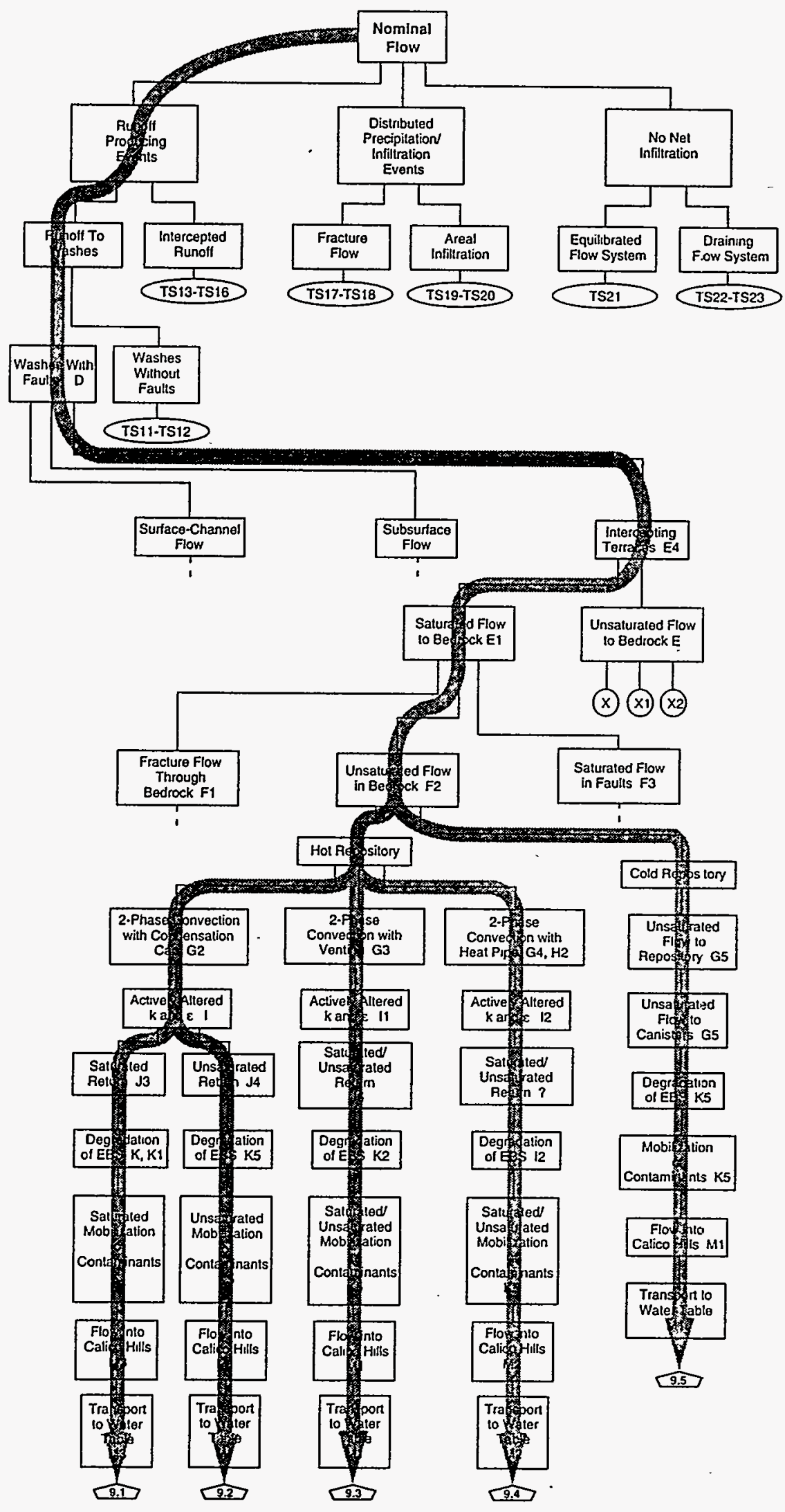

b. Scenario pathways, showing the relationship of the tree segment to the overall event tree for nominal flow. 


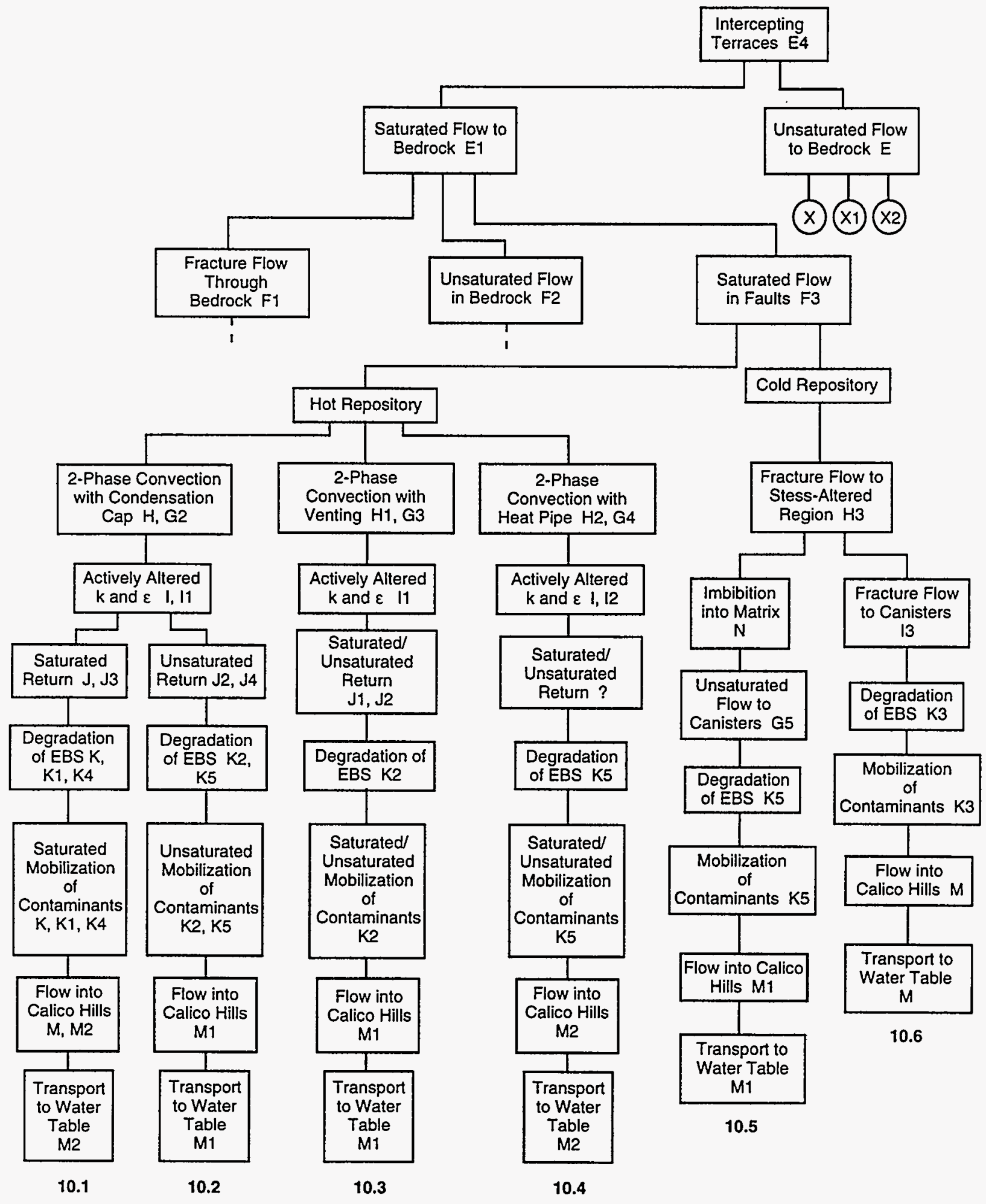

a. Events and their relationships, with cross-references to sketches illustrating each event.

Tree Segment 10. Nominal Flow, Runoff-Producing Events, Runoff to Washes, Washes With Faults, Intercepting Terraces, Saturated Flow to Bedrock, Saturated Flow in Faults. 


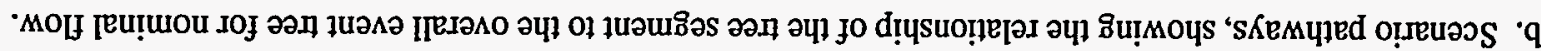

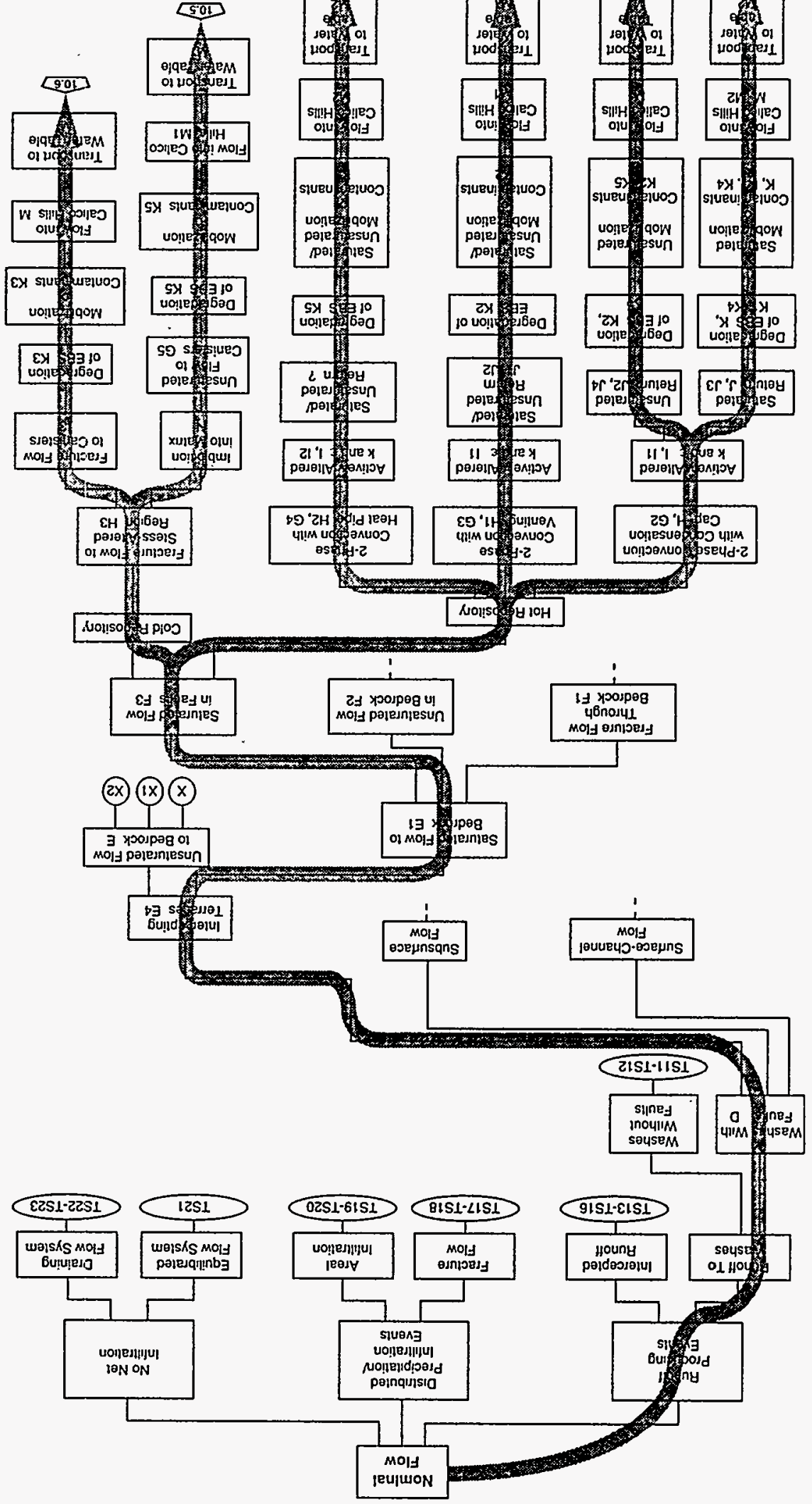




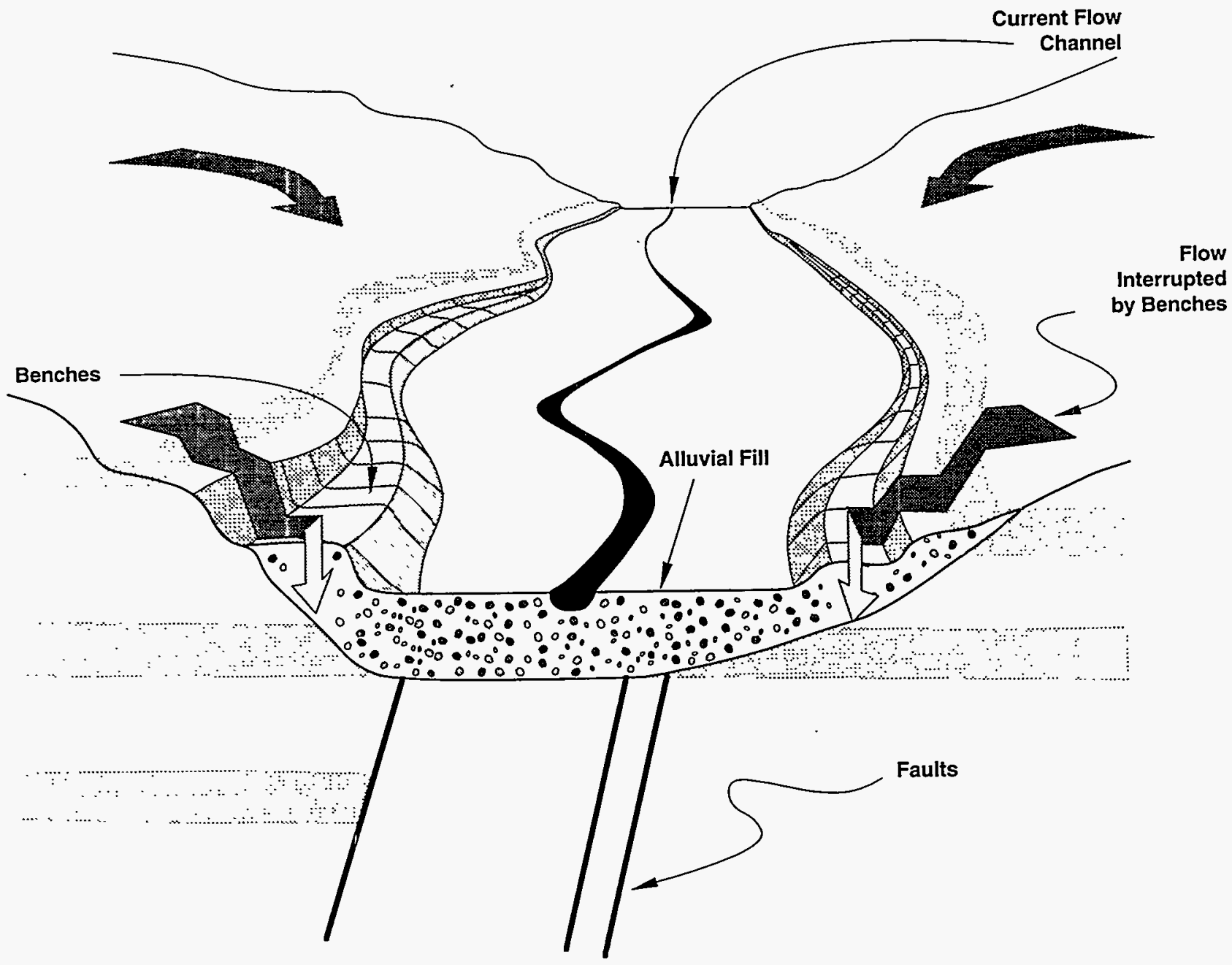

Sketch E4. Benches or terraces intercept runoff and direct it to a local flow system. 
The sequence of events below "Intercepting Terraces" and "Unsaturated Flow to Bedrock," E, is identical to Branches $\mathrm{X}, \mathrm{X} 1$, and $\mathrm{X} 2$ of Tree Segments 4,5 , and 6 , as indicated in the tree segments. This completes the discussion of washes with faults.

\subsubsection{Washes Without Faults}

Washes without faults differ from washes with faults in two important ways. First, of course, is the absence of controlling faults and stratigraphic offsets. Second, washes can now occur directly above waste containers; there is no reason to have an off-set from a fault zone. The expansions discussed above can be carried over for washes without faults, except for Branch Z5 from Tree Segment 3, which includes "Saturated Flow in Faults." Tree Segment 11 shows paths for a wash without faults over the repository with saturated flow to bedrock (Sketch E1, p. 25) and fracture flow through bedrock (Sketch F1, p. 27), and Tree Segment 12 shows paths for the case with saturated flow to bedrock and unsaturated flow in bedrock (Sketch F2, p. 28). Since all FEPs included in Branches $\mathrm{Z} 3$ and $\mathrm{ZA}$ have been discussed earlier, we will not repeat the explanations here. Surface-channel flow may instead be followed by unsaturated flow to bedrock (Sketch E, p. 24). The further development of this case is indicated in Tree Segments 11 and 12 as Branches $\mathrm{Z3}$ and $\mathrm{ZA}$.

There are two additional branches parallel to "Surface-Channel Flow" for this case of washes without faults (Figure 5, p. 107). The first, "Subsurface Flow in Wash" (Sketches E5 and E6) is followed by two branches-"Flow at Bedrock Contact" (Sketch E6) and "Flow in Alluvium" (Sketch E5). After this branching, both paths continue with Branches Z3 and Z4. For the second parallel branch, "Runoff Intercepted by Terraces" (Sketch C3) the branching is to "Saturated Flow to Bedrock" and "Unsaturated Flow to Bedrock," just as the case for "Surface Flow in Channels." These branchings are again followed by Branches $\mathrm{Z} 3$ and $\mathrm{ZA}$.

In washes without faults where the wash does not extend over the repository, fluid must migrate laterally from the area of infiltration to the repository, as for Drill Hole Wash. Arguments constructed for the washes without faults over the repository apply here with the lateral translation of the wash to outside the projection of the repository being imposed on all cartoons. As in the case of Drill Hole Wash, the source is weakened because fluids can miss the repository, even if they participate in the convective flow systems established by the repository. We again appeal to reference to earlier discussions to avoid repetition of the same arguments.

\subsection{Intercepted Runoff}

Runoff headed downslope to washes and off the mountain can be intercepted and diverted by exposed strata or by distributed surface infiltration. Diversion means that some part of the runoff infiltrates the mountain before arriving at the washes. Certain parts of the mountain have exposed strata that can accept fluids either in fractures or in pores. The Solitario Canyon face of Yucca Mountain and the slopes above several washes are examples of exposed strata. Distributed surface infiltration is the typical soil infiltration that occurs for soils with or without 


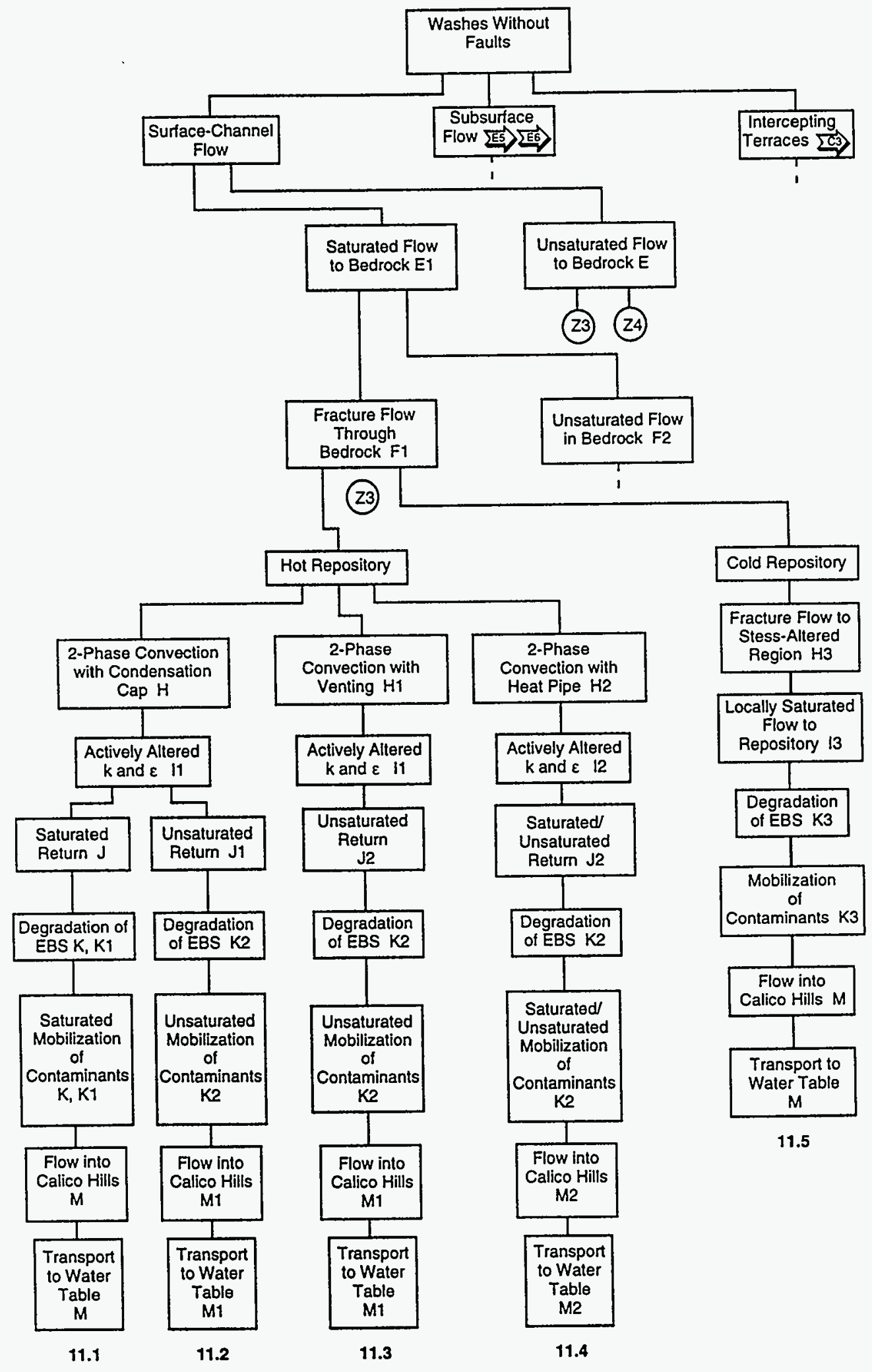

a. Events and their relationships, with cross-references to sketches illustrating each event.

Tree Segment 11. Nominal Flow, Runoff-Producing Events, Runoff to Washes, Washes Without Faults, SurfaceChannel Flow, Saturated Flow to Bedrock, Fracture Flow Through Bedrock. 


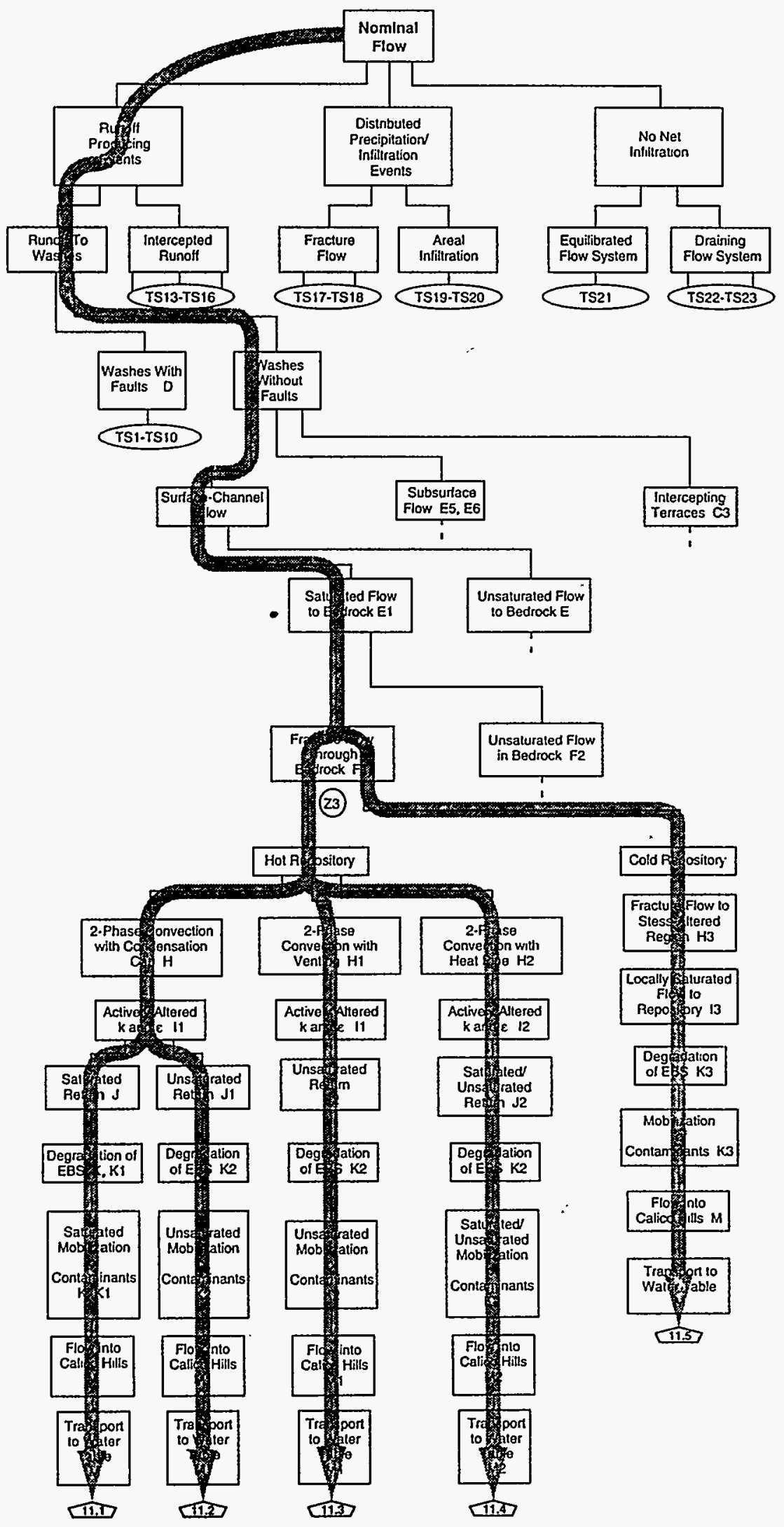

b. Scenario pathways, showing the relationship of the tree segment to the overall event tree for nominal flow. 


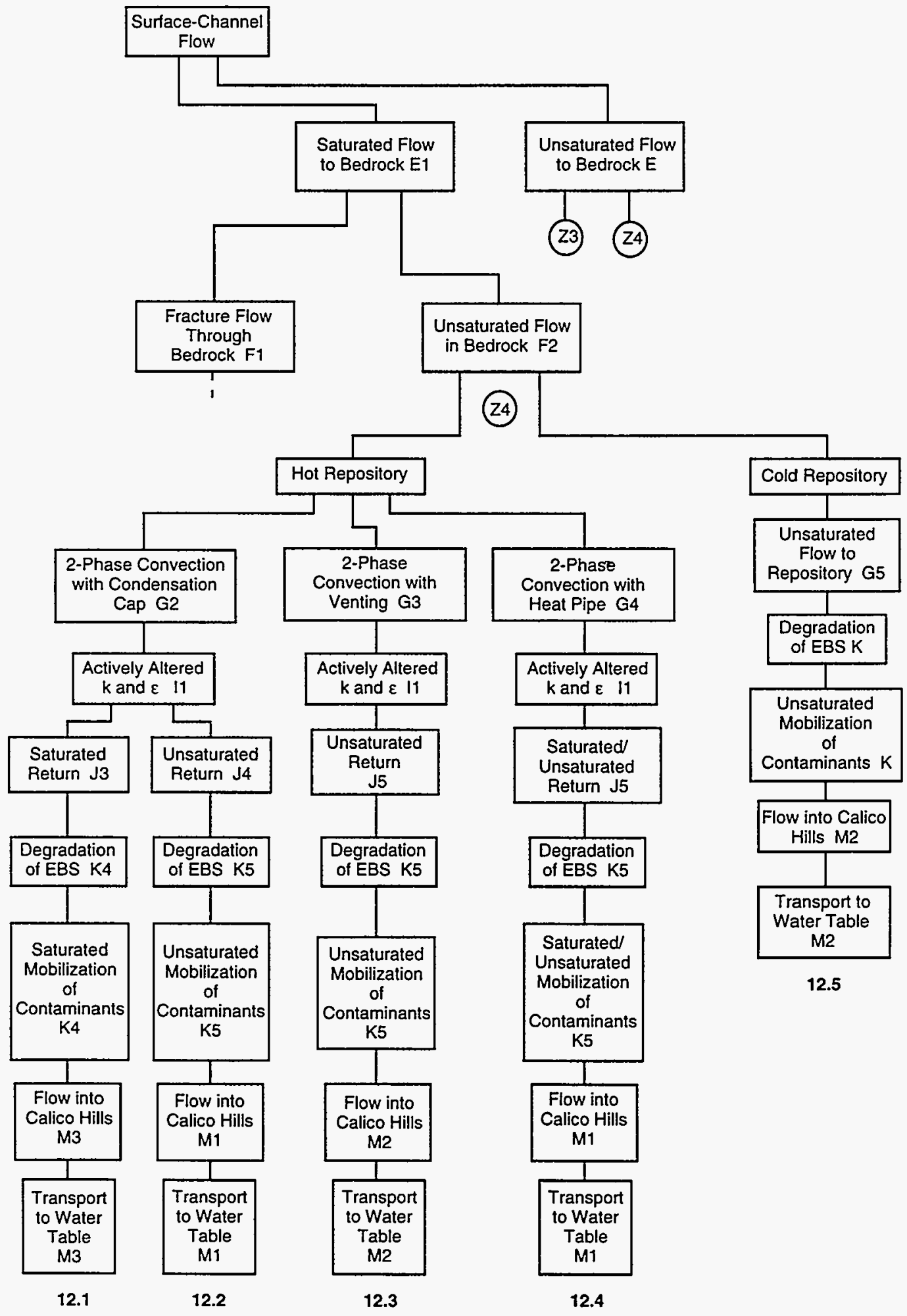

a. Events and their relationships, with cross-references to sketches illustrating each event.

Tree Segment 12. Nominal Flow, Runoff-Producing Events, Runoff to Washes, Washes Without Faults, SurfaceChannel Flow, Saturated Flow to Bedrock, Unsaturated Flow in Bedrock. 


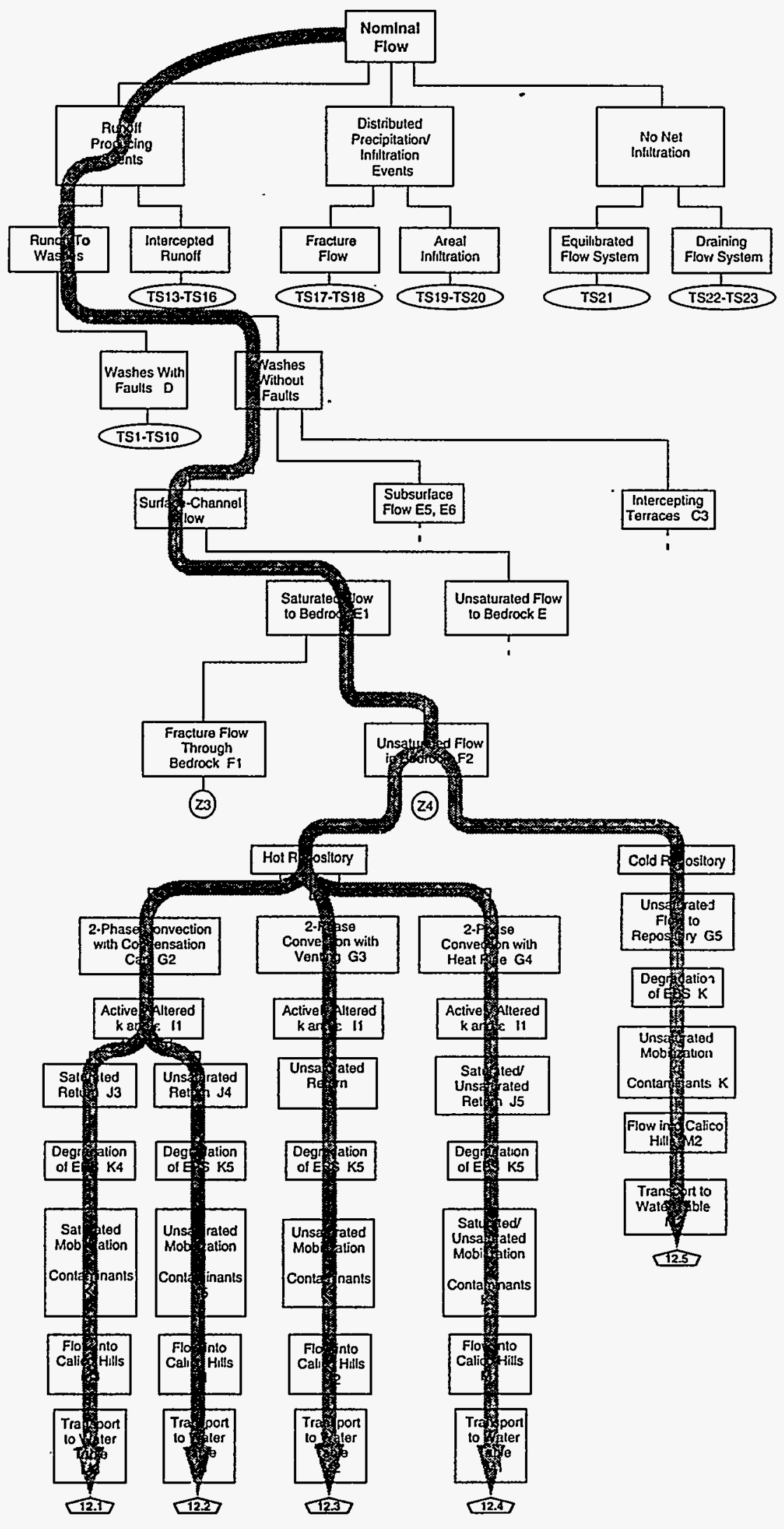

b. Scenario pathways, showing the relationship of the tree segment to the overall event tree for nominal flow. 


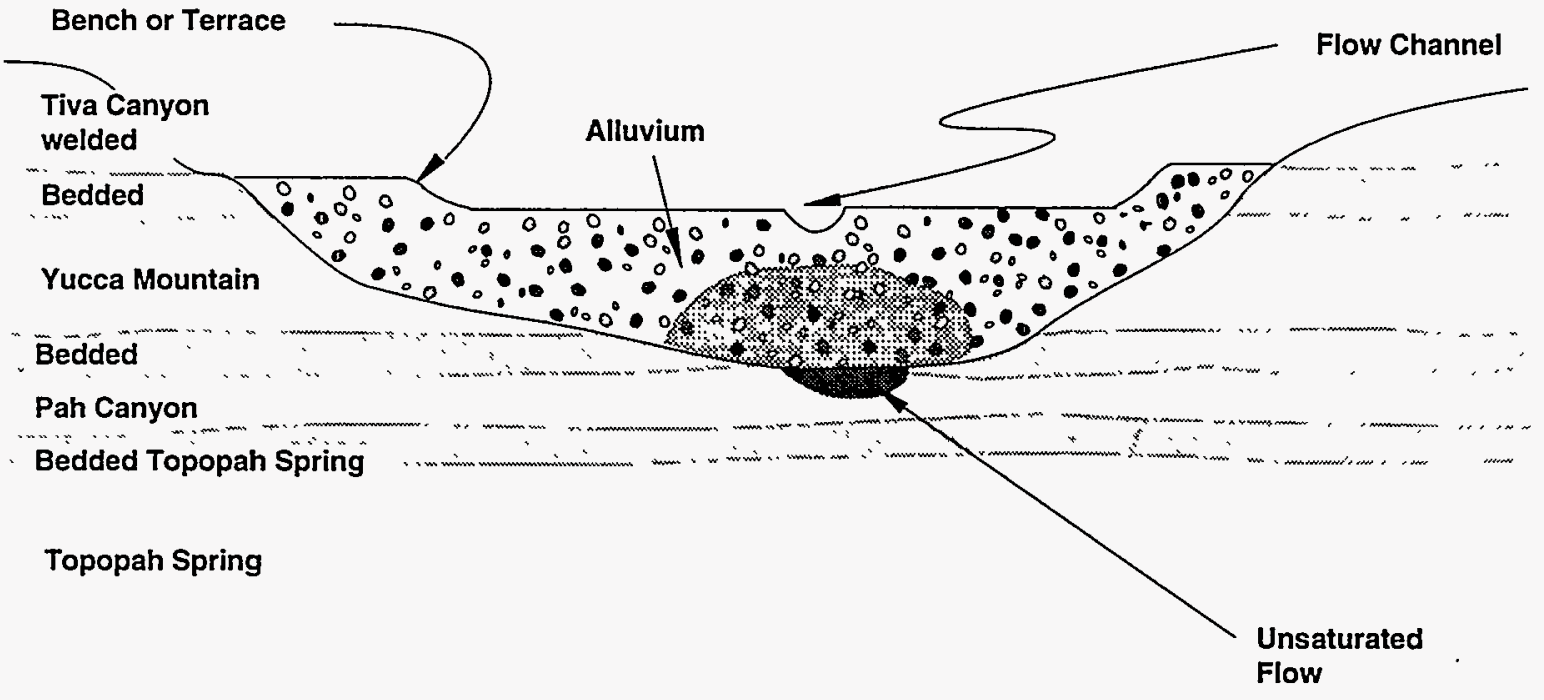

Sketch E5. Flow in alluvium in a wash without faults. 


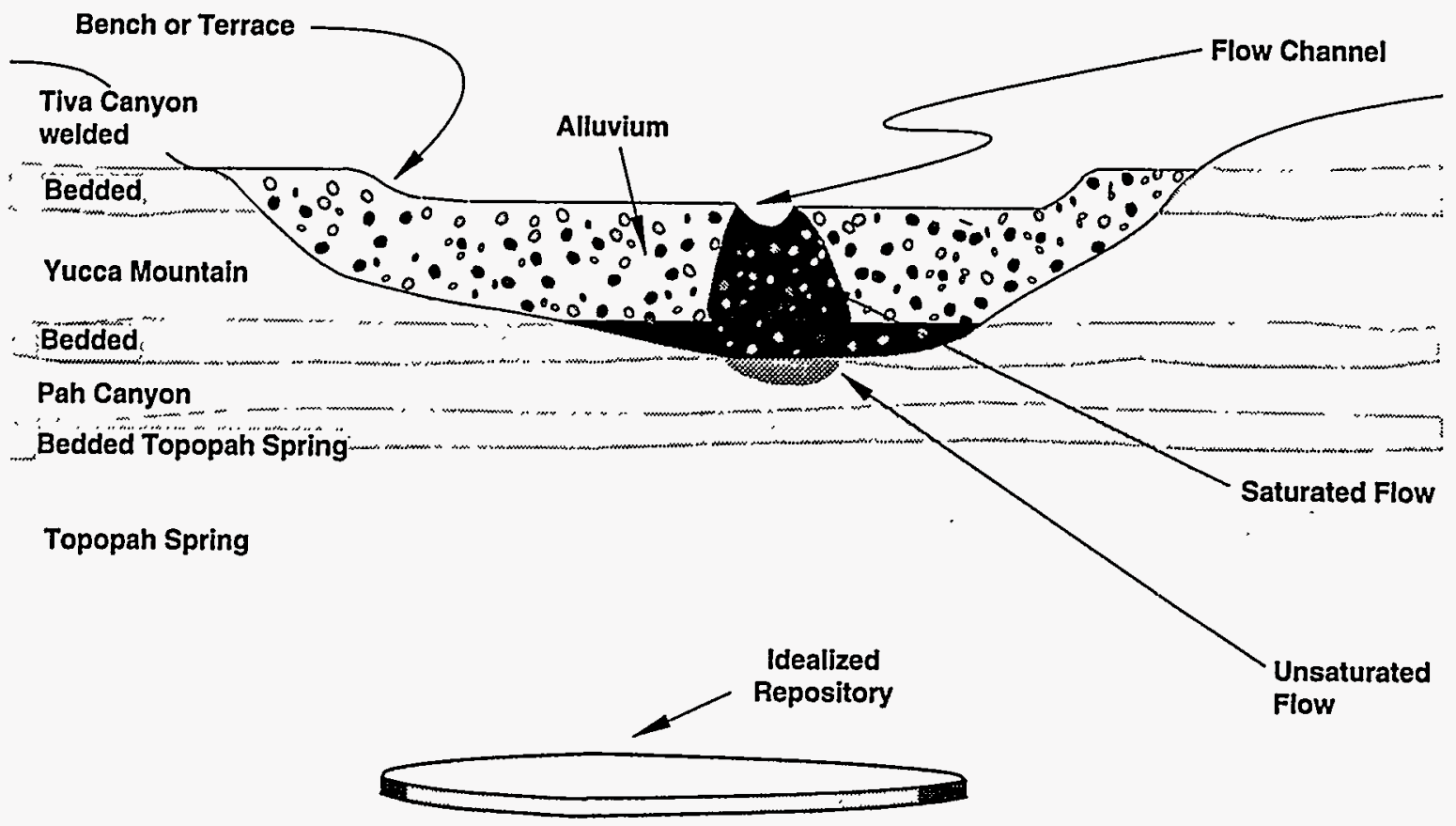

Sketch E6. Flow at bedrock contact in a wash without faults. 


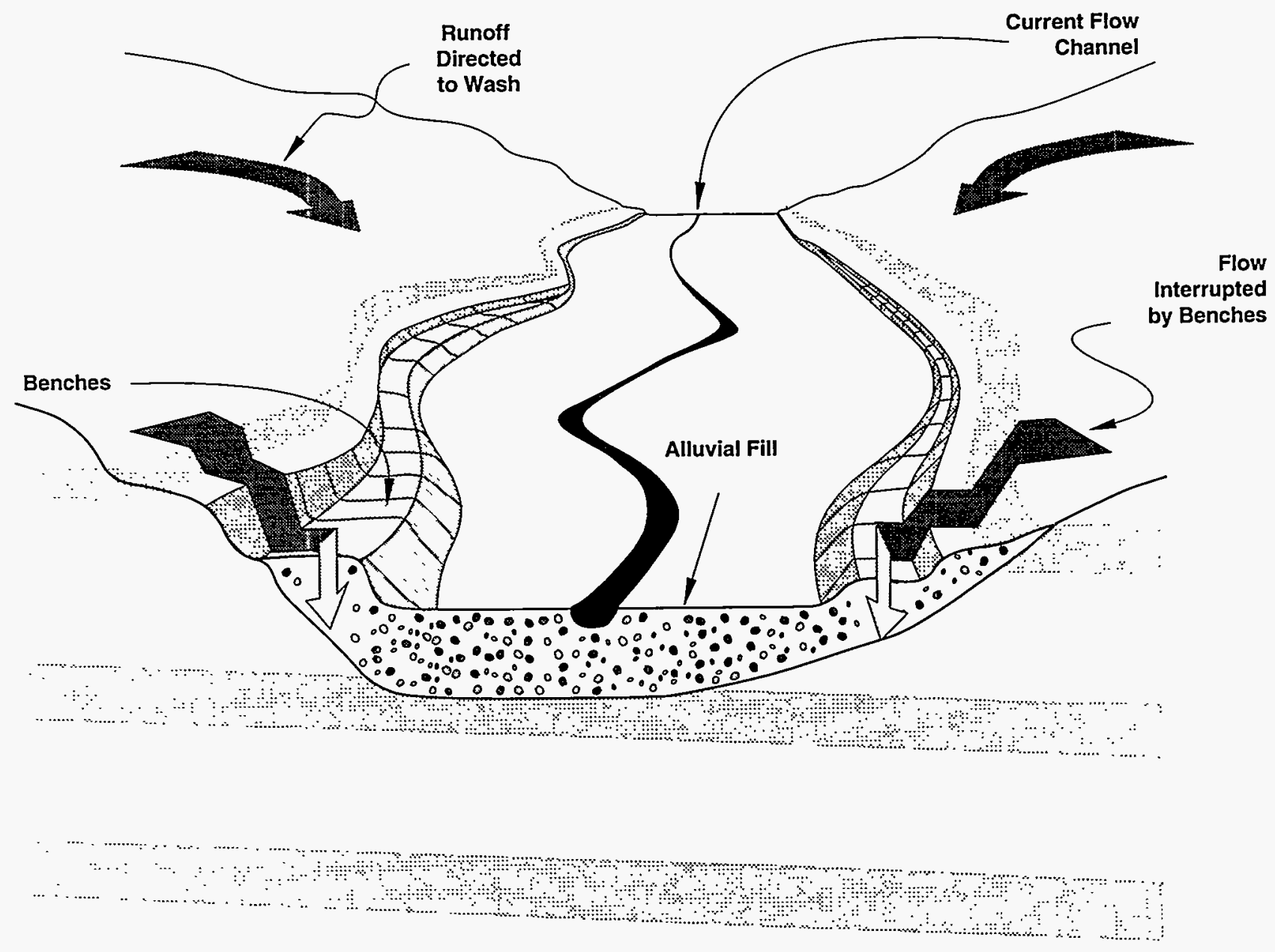

Sketch C3. Runoff intercepted by benches or terraces in a wash without faults. 


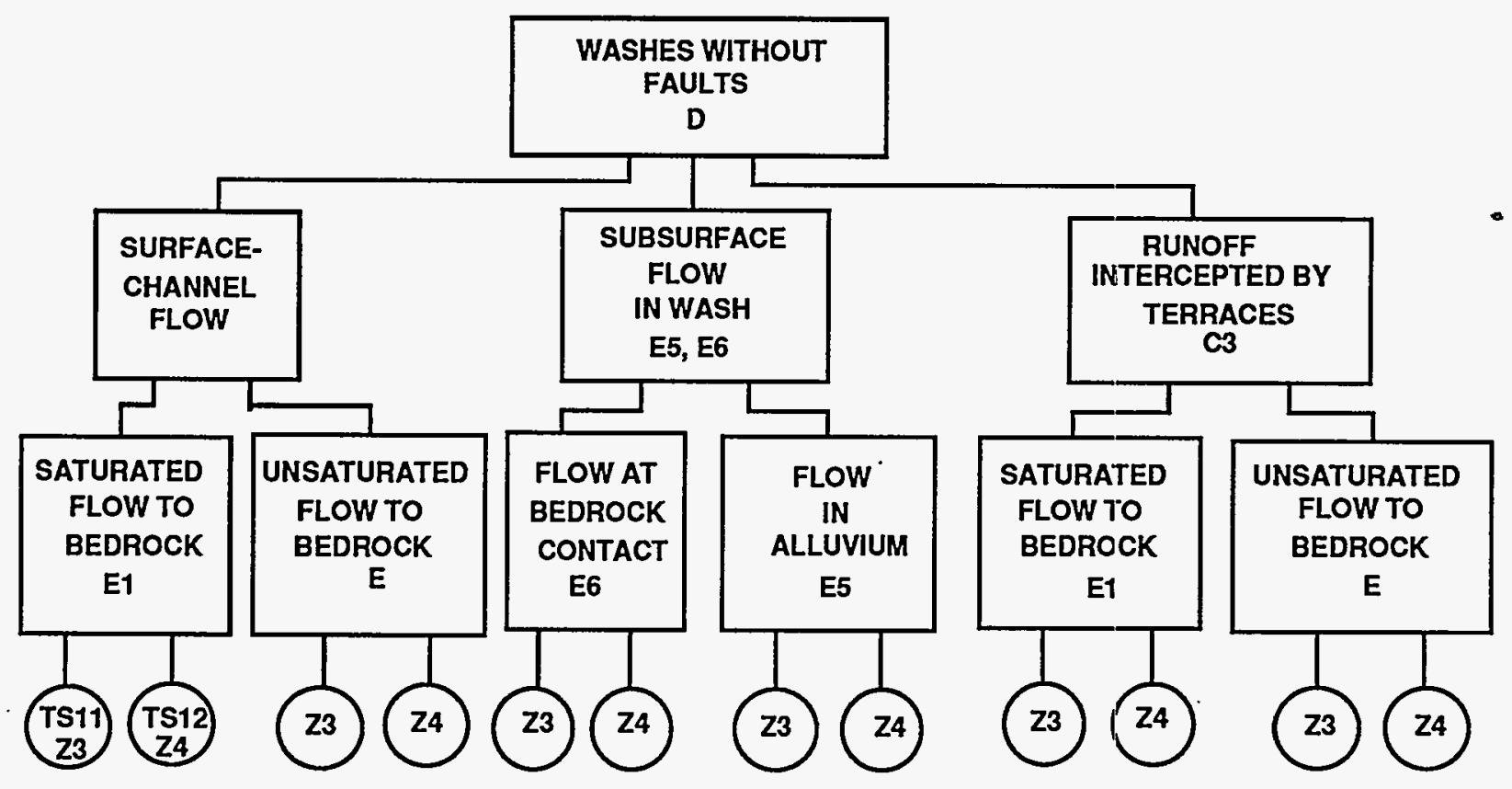

Figure 5. Intermediate layers of the event tree for nominal flow, showing major branches for washes without faults.

vegetative cover. Soil here includes decomposed rock with little or no organic content. Tree Segment 13 contains Paths $\widehat{13.1}$ through $\widehat{13.6}$, all involving interception of flow by exposed strata. The basic idea is illustrated by Sketches A and A1.

Early site characterization revealed very recent water in a borehole that apparently intersected a fracture in the Topopah Spring units. This recent water was adjacent to and above much older water. The explanation has been that the recent water was the product of rapid transit down fractures to its current location. Calculations on fracture flow (Nitao and Buscheck, 1989a, 1989b; Nitao, 1989) indicate that rapid movement is possible without imbibition in some circumstances. There were special circumstances surrounding the recent water in the Topopah Spring units borehole-namely that the flow path depends on the exposed strata on the west side of the repository block (Sketch A2). What is important for these discussions is the water's apparently rapid movement to the Topopah Spring units and its subsequent current immobility. In addition, the exposed strata could intercept flow in much shorter, local fractures or directly into the pores of the rock. Tree Segments 13 and 14 (p. 114) recognize these possibilities; their relative importance would have to be determined experimentally. 


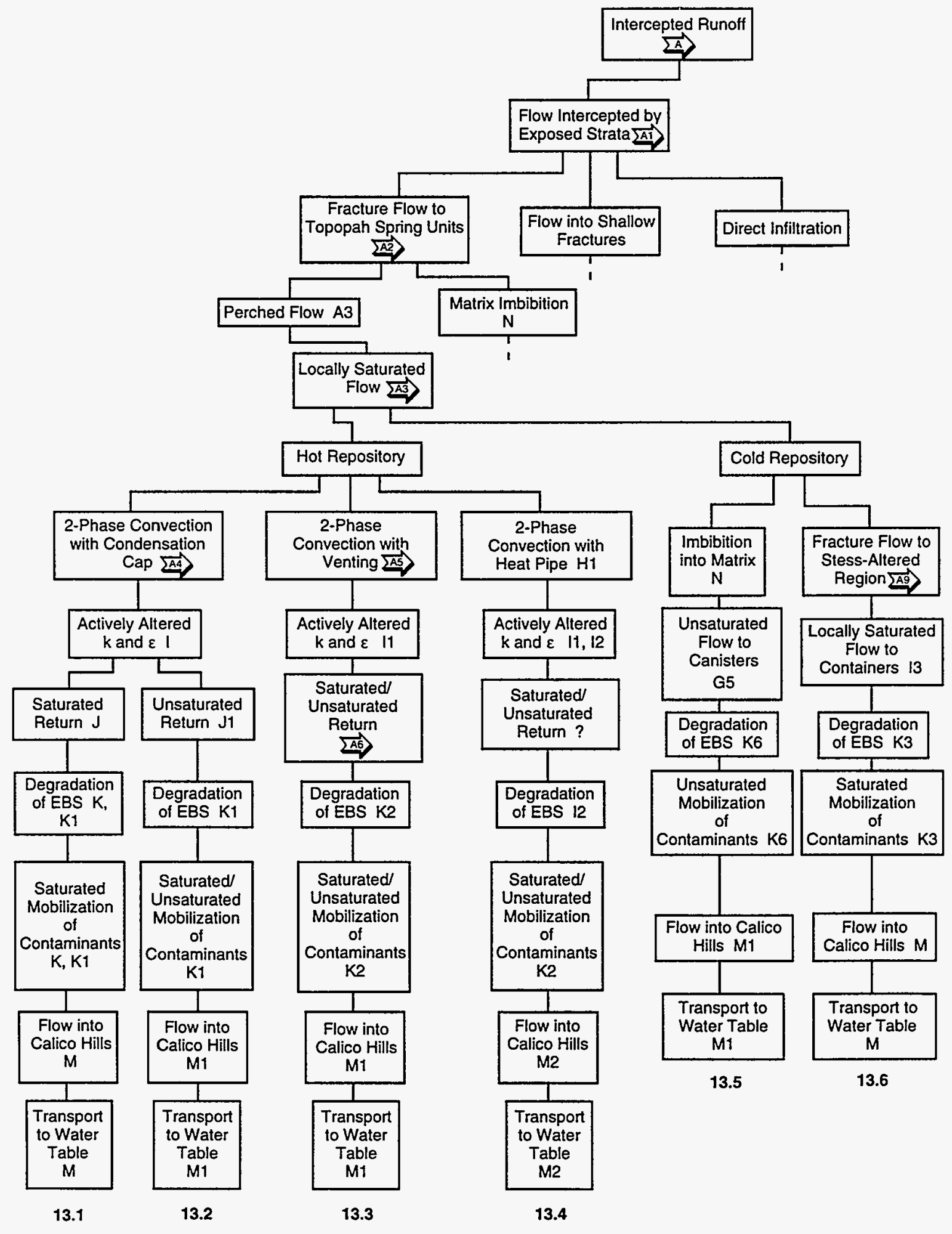

a. Events and their relationships, with cross-references to sketches illustrating each event.

Tree Segment 13. Nominal Flow, Runoff-Producing Events, Runoff Intercepted, Flow Intercepted by Exposed Strata, Fracture Flow to Topopah Spring Units, Perched Flow. 


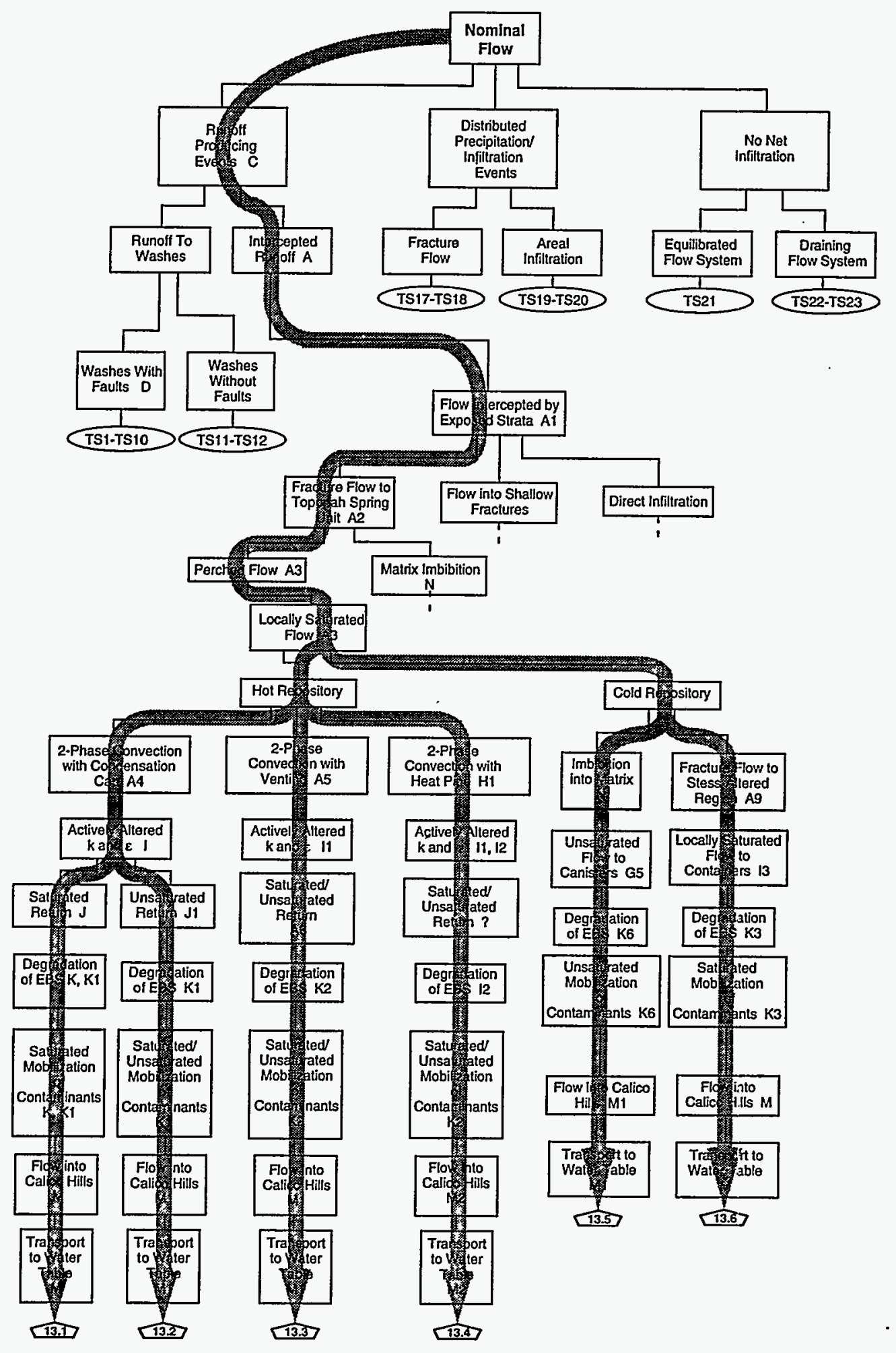

b. Scenario pathways, showing the relationship of the tree segment to the overall event tree for nominal flow. 


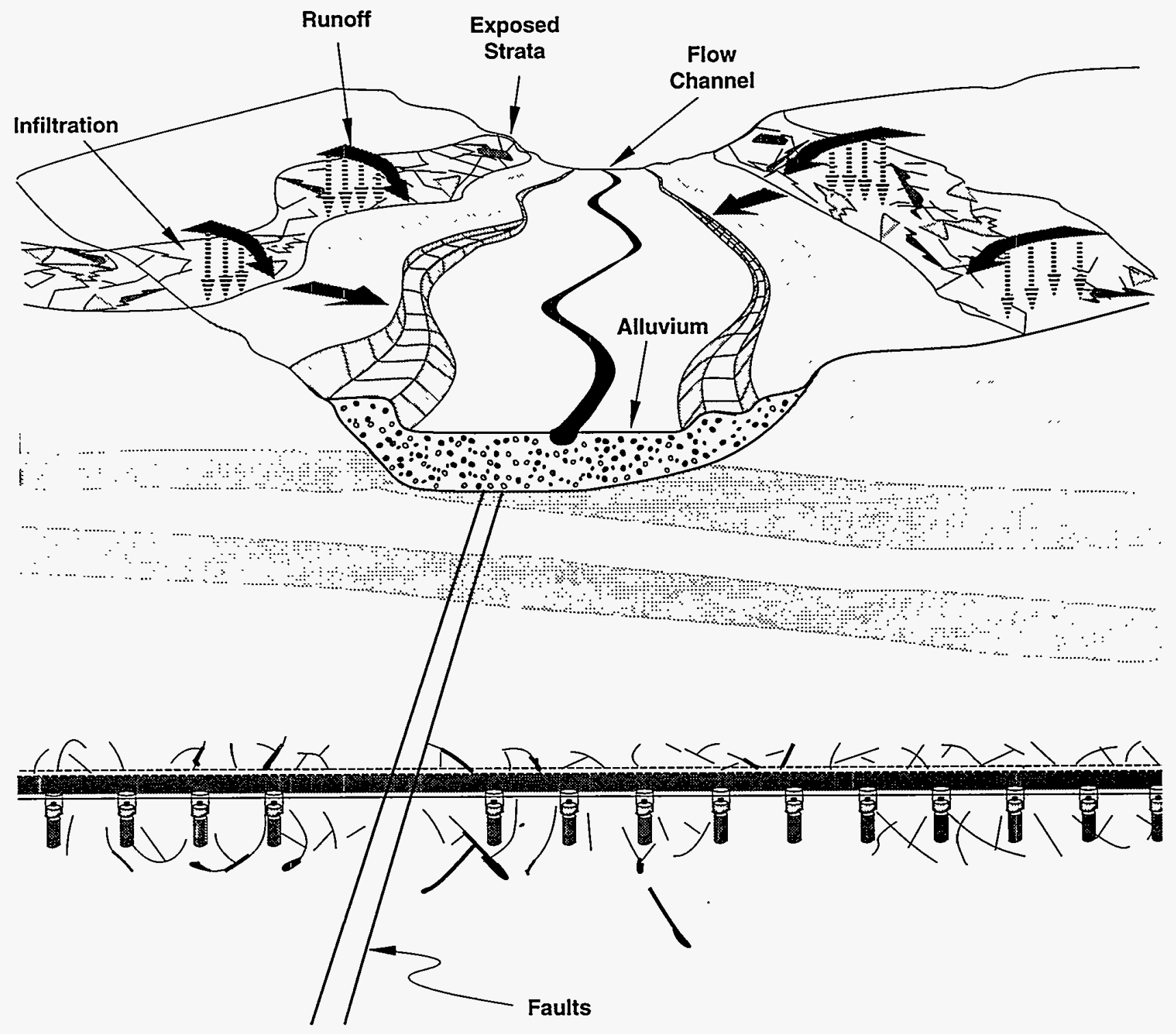

Sketch A. Runoff intercepted and diverted by exposed strata before reaching a wash. 


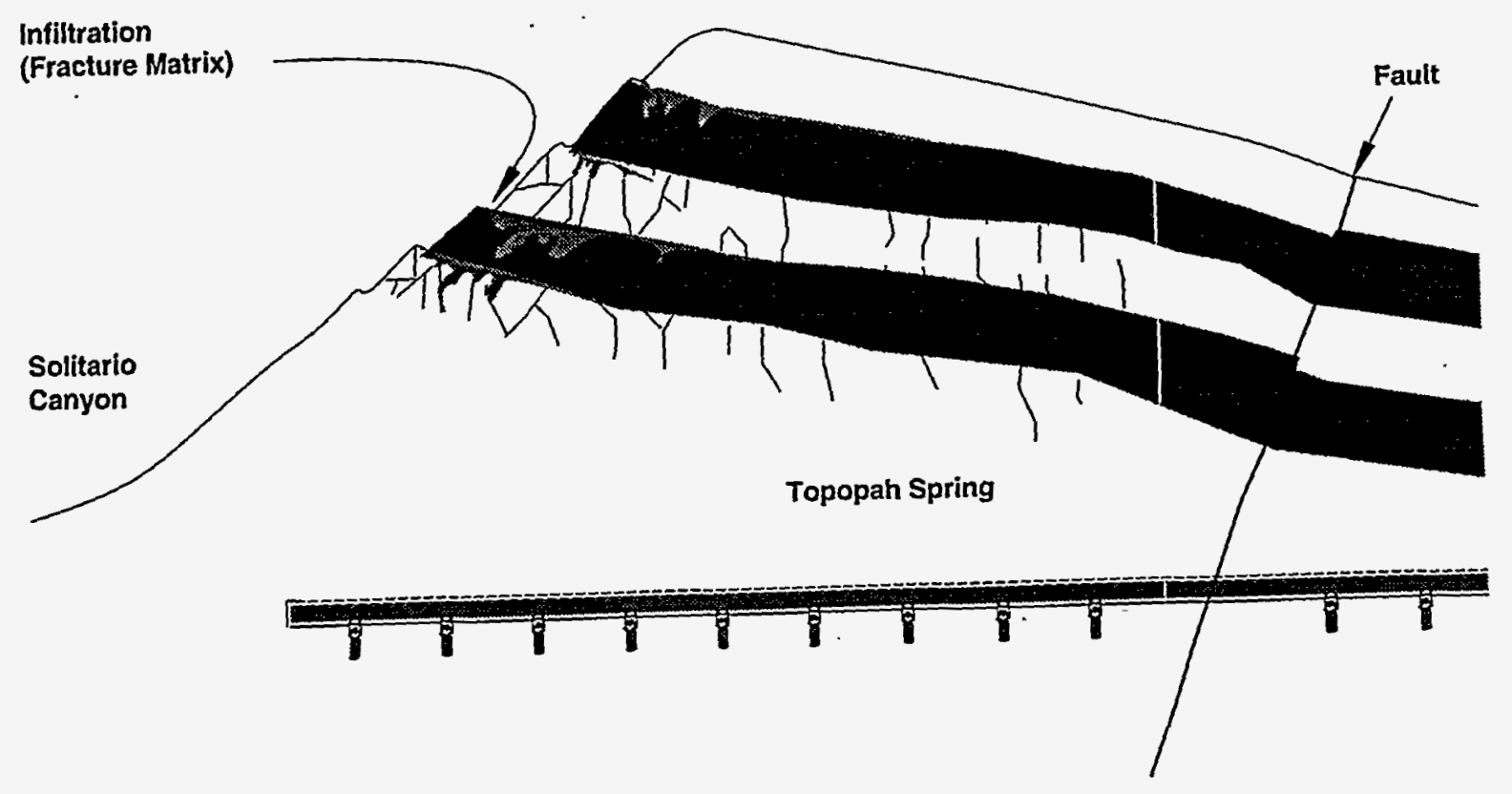

Sketch A1. Runoff intercepted and diverted by exposed strata before reaching Solitario Canyon. 


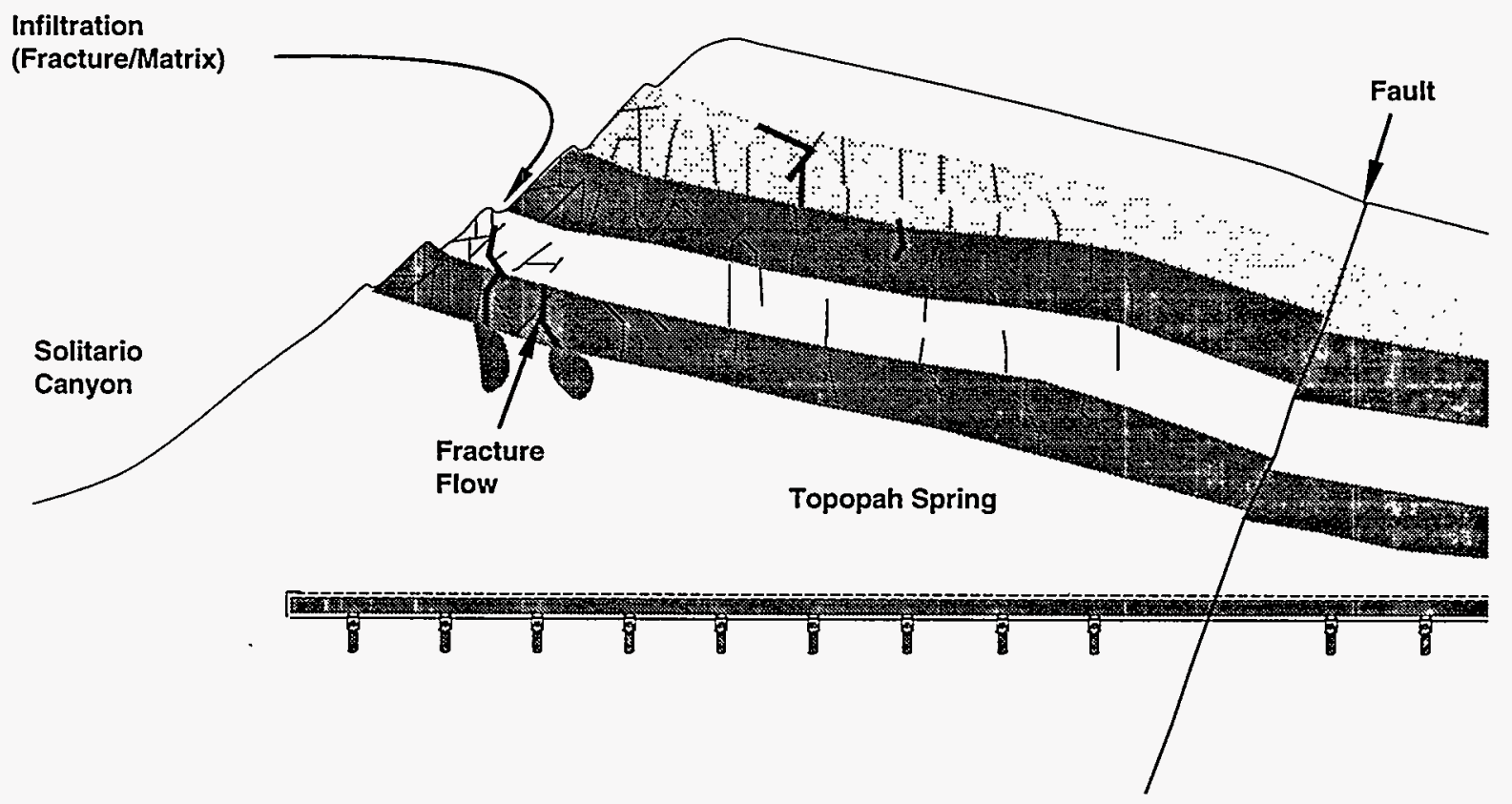

Sketch A2. Intercepted runoff flowing in fractures to the Topopah Spring Units. 


\subsubsection{Fracture Flow to Topopah Spring Units (Perched Flow)}

If fracture flow to the Topopah Spring units is established, the flow system is close enough to the repository to interact with the thermal field established by the waste or with its long-term residual thermal perturbation. Further expansion of the event tree gives us Paths 13.1] through 13.6.

Sketch A3 illustrates a possible means by which rapid flow down a fracture could end up as perched water. In this sketch, a fracture ends at a stratum boundary. Liquid moves along that boundary to a fault and then moves down the fault. The sketch shows the fault close to the fracture, but it could be any distance away. This is only one possibility for a connected path; many others might be constructed. Six branches occur in the tree at this point, four for two-phase flow around a hot repository and two for a cold repository.

\subsubsection{Hot Repository, Paths 13.1 Through 13.4}

We will discuss the four hot branches first, beginning with the branch allowing for a condensation cap (Sketch A4). The perched zones are a source of additional fluid (Sketch A3). The exact location of the condensation cap depends on flow rates; the return paths from the cap to containers depend on alterations in permeability and porosity caused by precipitation (Sketch I, p. 15). The remainder of this scenario is like Path $\widehat{\Upsilon 1.1}$. Path $\widehat{13.1}$ has led us to the construction of the scenario described in Sketches $\mathrm{C}, \mathrm{A}, \mathrm{A} 1, \mathrm{~A} 2, \mathrm{~A} 3, \mathrm{A4}, \mathrm{I}, \mathrm{J}, \mathrm{K}, \mathrm{K} 1$, and $\mathrm{M}$.

If the return flow, reflux from the condensation cap, is unsaturated/saturated flow (not fracture flow), the tree calls it unsaturated return and distinguishes it from fracture flow reflux because the volumetric rate and the exposure of the waste to fluid would be much different. We expect these differences to produce substantially different computational requirements in modeling. Path 13.2 examines this unsaturated return in $\mathrm{C}, \mathrm{A}, \mathrm{A1}, \mathrm{A} 2, \mathrm{~A} 3, \mathrm{~A} 4, \mathrm{I}$, $\mathrm{J} 1, \mathrm{~K} 1, \mathrm{M} 1$.

The next hot repository branch, Path [13.3, is for two-phase convective flow with venting. There is no condensation cap, so downward flow from a zone of perched water (Sketch A3) acts as a continuing source feeding the vaporization isotherm (Sketch A5). The repository might act as a thermal pump, vaporizing fluid reaching the vaporization isotherm and driving it out of the mountain. The size of the dryout zone depends on the thermal load and the size of the fluid sources (fractures and possibly the water table). The dryout zone (and perhaps the region around it) is expected to have its hydraulic properties altered by precipitates (Sketch I1, p. 36). The vaporization isotherm will follow the flow of steam into fractures, producing a somewhat erratic form. Return flow to the repository will occur primarily when waste containers have cooled sufficiently to put the vaporization isotherm inside the waste containers or their remains. Such return flow is from flow down fractures, from unsaturated flow from cooler rock, and from vapor flow from unsaturated rock surrounding the repository. The return is sketched in Sketch A6. Corrosion and degradation of the EBS occur both during the hot period, when the EBS is exposed to hot vapor, and during the cold period when pores are refilling and fractures can conduct fluid to the waste containers. Sketch K2 (p. 43) represents these processes, although what is actually shown is movement of fluids to and from the 


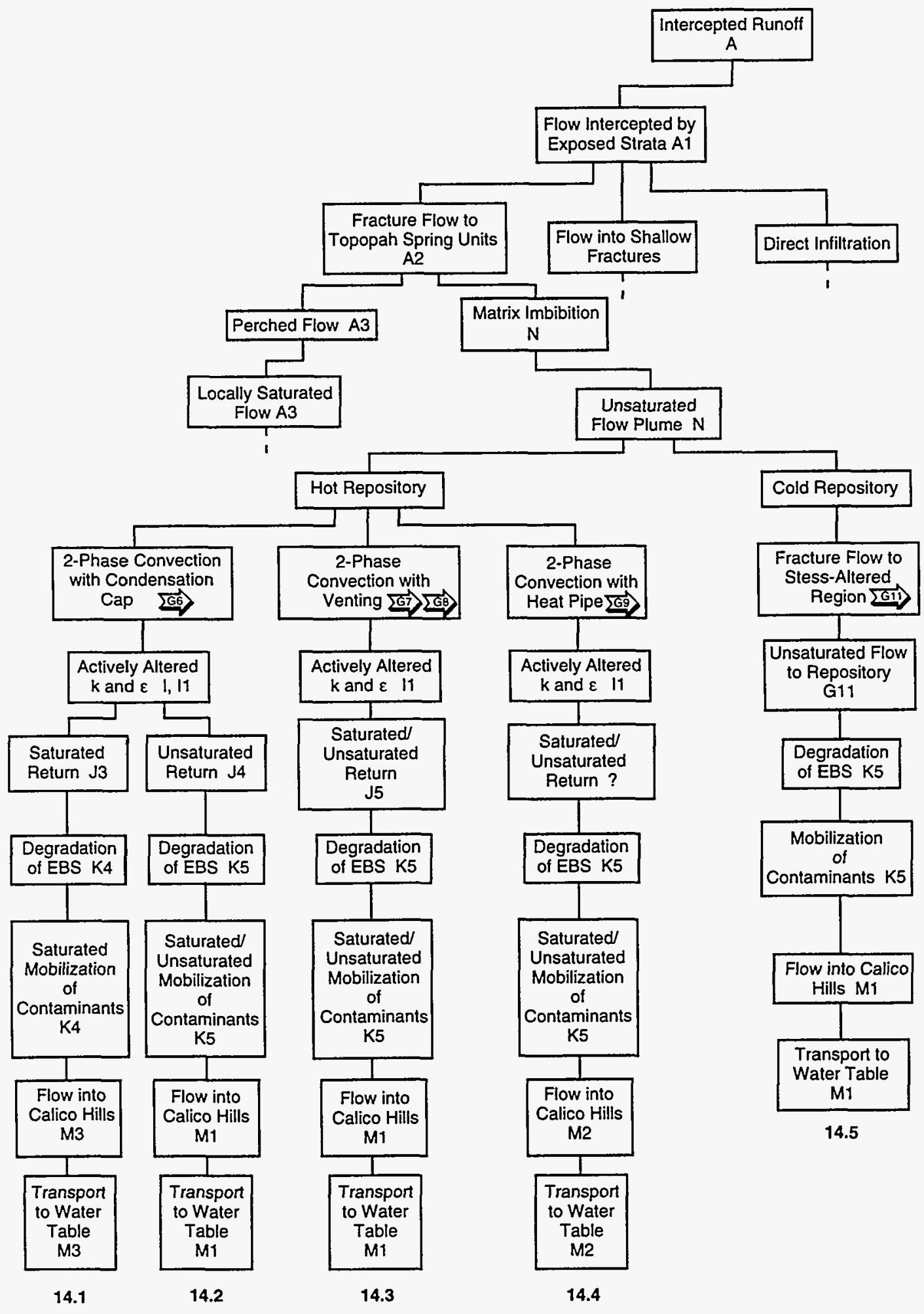

a. Events and their relationships, with cross-references to sketches illustrating each event.

Tree Segment 14. Nominal Flow, Runoff-Producing Events, Runoff Intercepted, Flow Intercepted by Exposed Strata, Fracture Flow to Topopah Spring Units, Matrix Imbibition. 


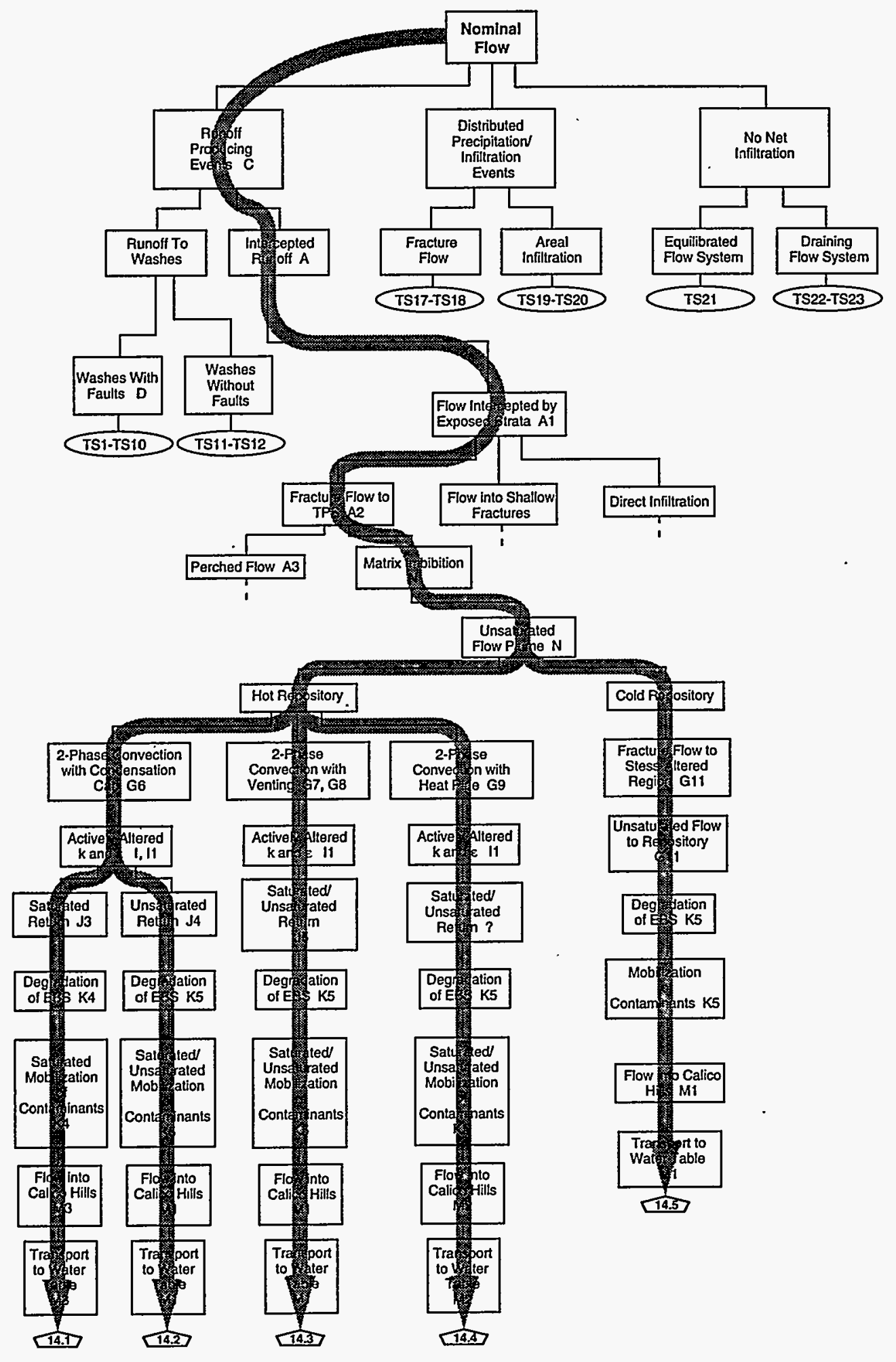

b. Scenario pathways, showing the relationship of the tree segment to the overall event tree for nominal flow. 


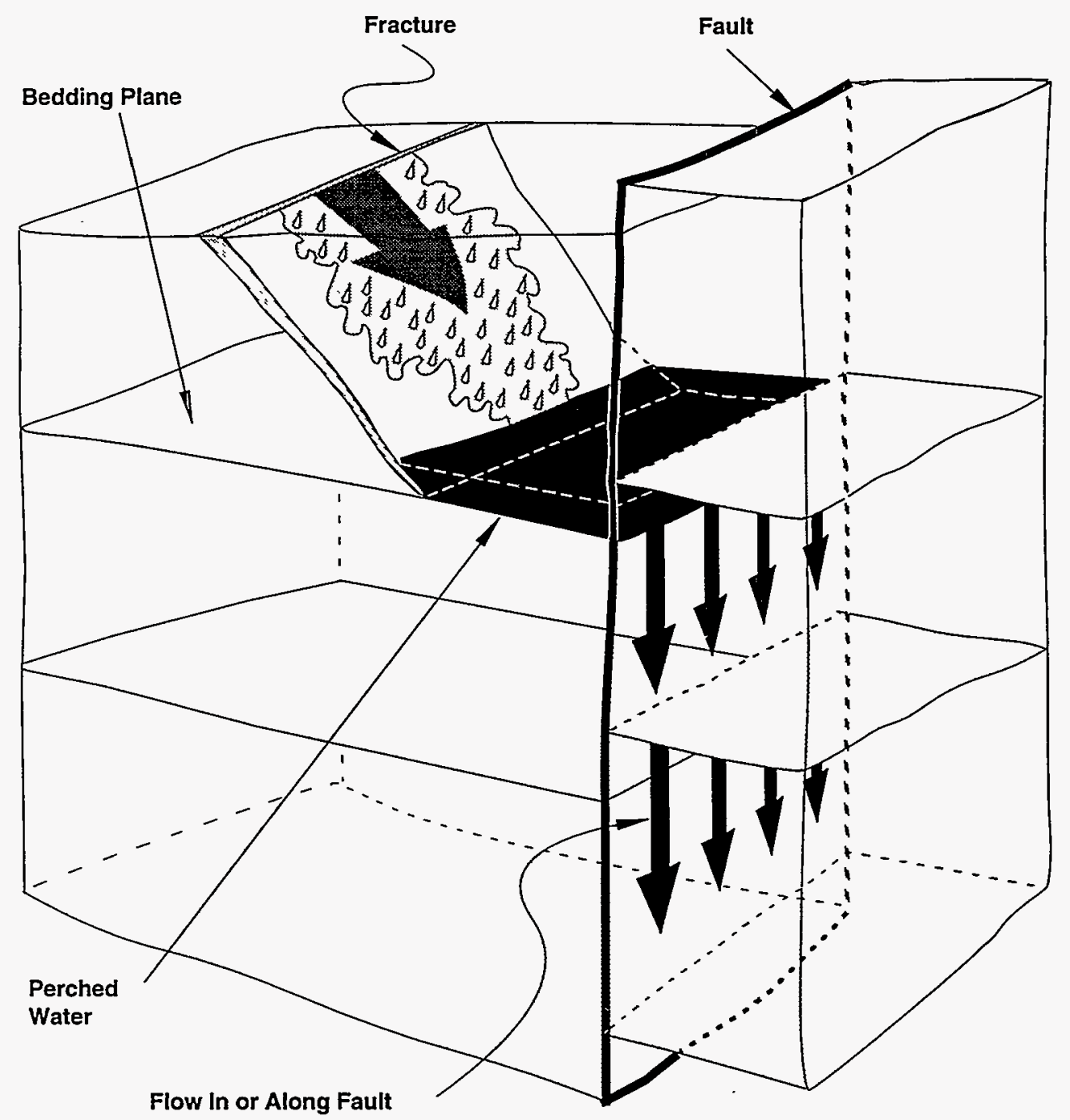

Sketch A3. Zone of perched water formed by rapid flow down a fracture. 


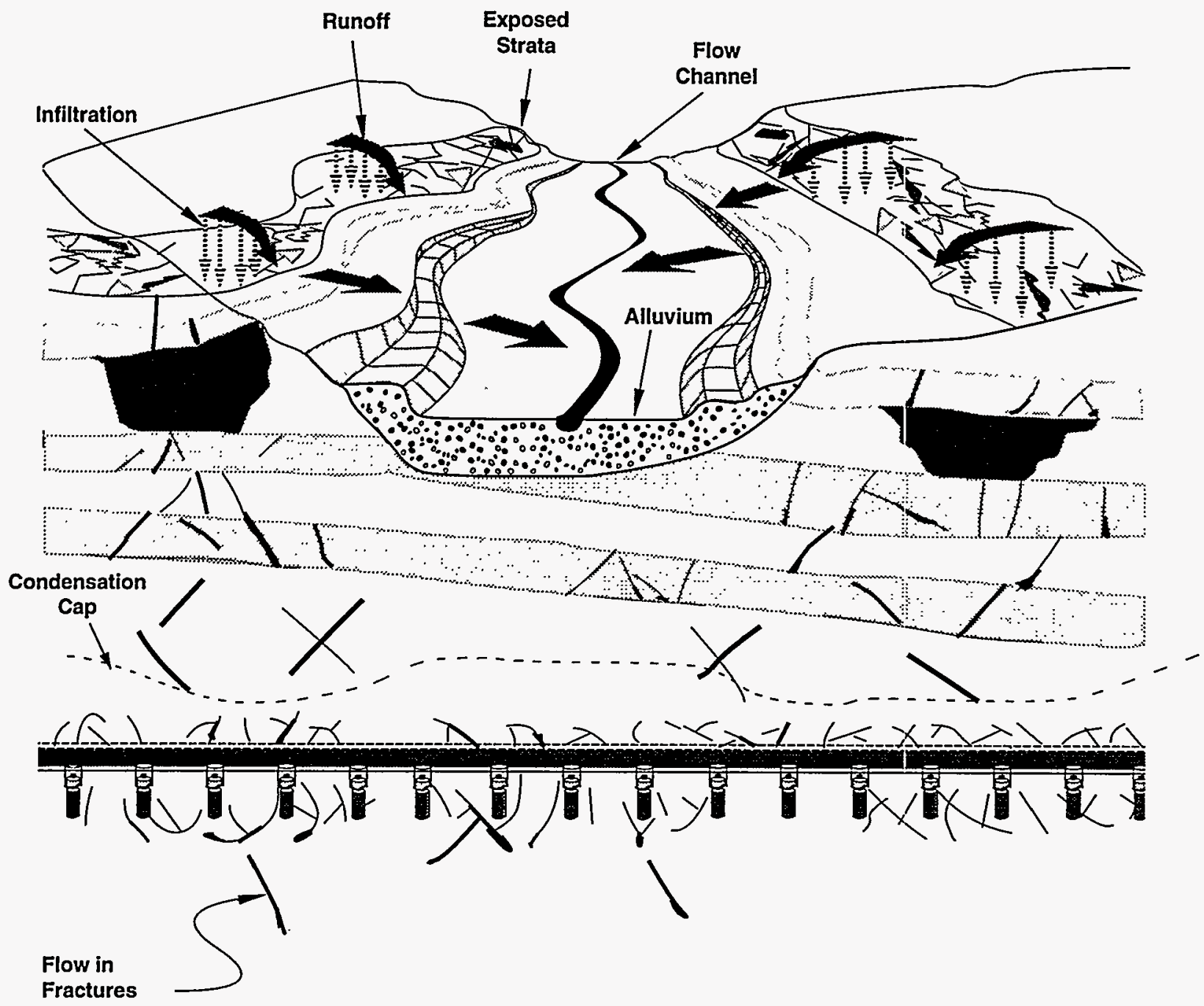

Sketch A4. Perched fluids interacting locally with the condensation cap. 


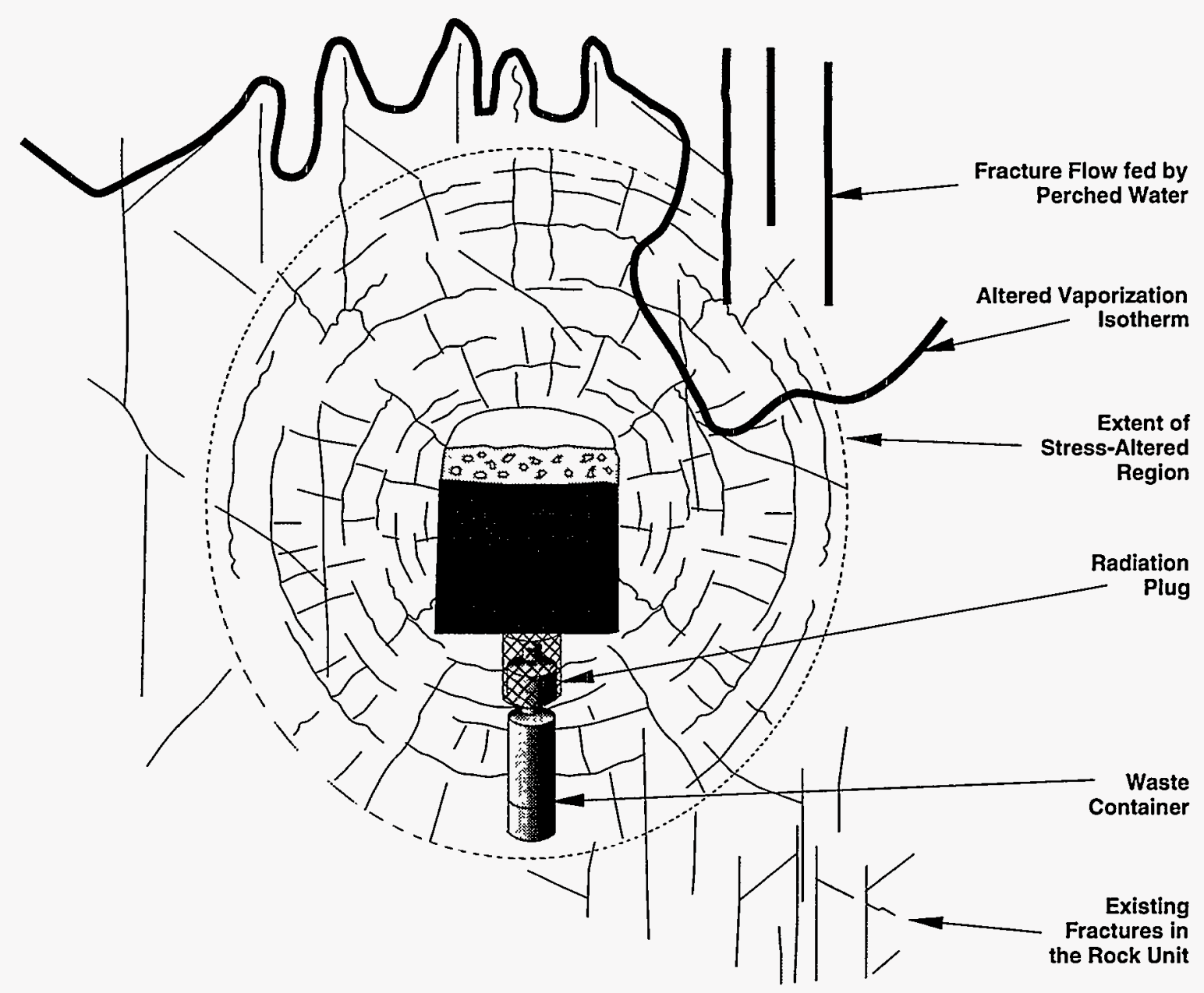

Sketch A5. Perched water acting as a continuing source of liquid at the vaporization isotherm. 


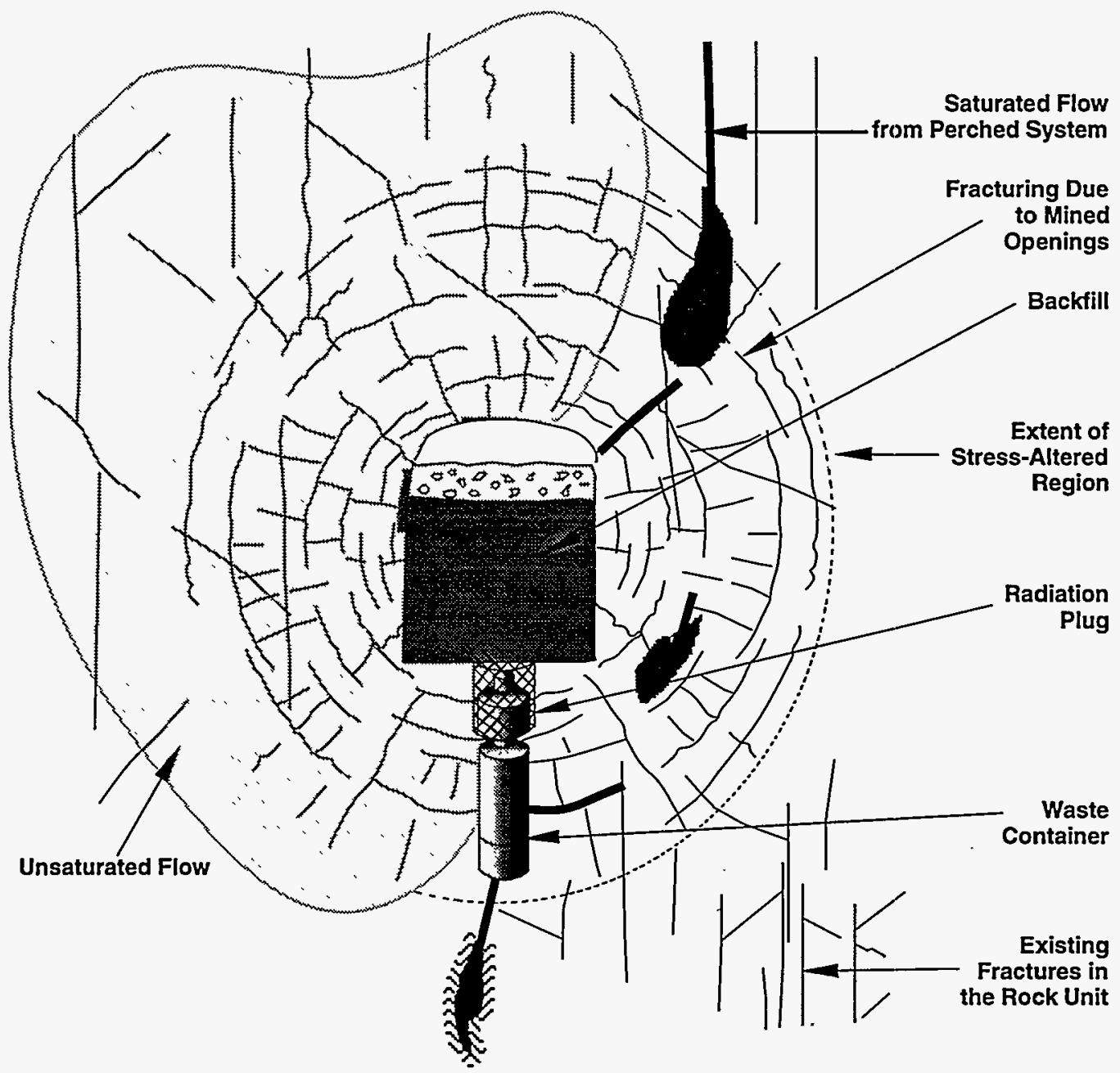

Sketch A6. Unsaturated and perched flow reaching the repository. 
container. Actual behavior of the container as a function of temperature, fluid volume, saturated or unsaturated flow, and waste type is currently under study. Mobilization of contaminants is depicted in Sketch K2; whether mobilization is in a saturated or an unsaturated flow system depends on the volumetric rate for return and on the altered hydraulic properties of the rock. Flow into the Calico Hills units and down to the water table is fed by the unsaturated return flow after cooling and by fracture flow from the perched source (Sketch M1, p. 44). Scenario C, $\mathrm{A}, \mathrm{A} 1, \mathrm{~A} 2, \mathrm{~A} 3, \mathrm{~A} 5, \mathrm{I1}, \mathrm{A} 6, \mathrm{~K} 2$, and $\mathrm{M1}$ describes this path, Path $\widehat{13.3}$.

The fourth path driven by the thermal output of the repository, Path $\widehat{13.4}$, involves two-phase convective flow in a heat pipe. The locally saturated flow from the perched system (above the heat pipe in the tree) acts as a source, probably episodic, possibly continuous, of new fluid for the two-phase process. During the operation of the heat pipe, one expects that evaporation will alter hydraulic properties by precipitation and that movement of steam past the container will cause some corrosion (see Sketch I2, p. 50). We expect little movement of contaminants while the heat-pipe phase lasts; once cool, the containers will see the reestablished unsaturated flow system and saturated fracture flow (Sketch K2). Contaminants move to the Calico Hills units and on to the water table by unsaturated flow, as reestablished, and by locally saturated flow driven by the saturated fracture flow from the perched system above. The scenario is described by Sketches $\mathrm{C}, \mathrm{A}, \mathrm{A} 1, \mathrm{~A} 2, \mathrm{~A} 3, \mathrm{H1}, \mathrm{I1}, \mathrm{I} 2$, ?, K2, and M2.

\subsubsection{Cold Repository, Paths $\widehat{13.5}$ and 13.6}

Fluids directed to the repository by locally saturated flow (Sketch A3) could be imbibed by the matrix and travel to the waste as an unsaturated flow plume (Sketches $N$ and G5, pp. 53 and 54), as in Path 1.5 . Path 13.5 includes $\mathrm{C}, \mathrm{A}, \mathrm{A1}, \mathrm{A} 2, \mathrm{~A} 3, \mathrm{~N}, \mathrm{G} 5, \mathrm{~K} 6$, and $\mathrm{M} 1$.

Path $\widehat{13.6}$ for a cold repository starts with flow to the stress-altered region that forms around the drift (Sketch A9). Runoff intercepted on its course downhill and redirected to the stress-altered region is shown schematically in Sketch A9. The remainder of this scenario is like Path 1.6 . Path 13.6 is the scenario described by Sketches C, A, A1, A2, A3, A9, I3, K3, and M.

\subsubsection{Fracture Flow to Topopah Spring Units (Matrix Imbibition)}

In addition to the path that described perched flow resulting from fracture flow to the Topopah Spring units, we have paths for unsaturated flow produced from this same source-fracture flow to the Topopah Spring units (Sketch

N), Paths $\widehat{14.1}$ through $\widehat{14.5}$ in Tree Segment 14. Sketch $N$ depicts the fate of flow down fractures that come to a dead end. Such flow leaks from the fractures to the rock pores and becomes part of the unsaturated flow system. 


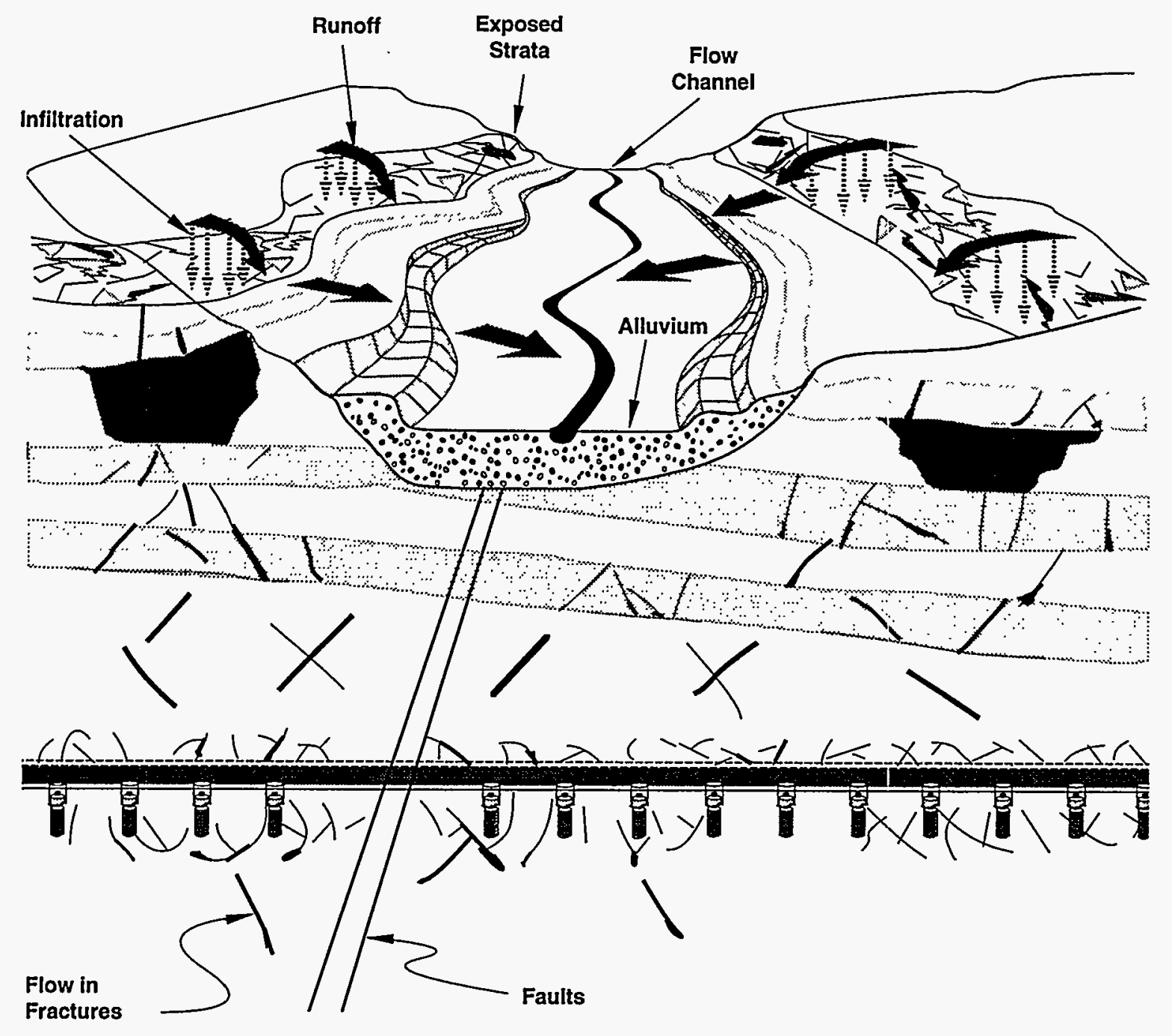

Sketch A9. Intercepted runoff being conveyed to the repository as fracture flow. 


\subsubsection{Hot Repository, Paths 14.1 Through $\widehat{14.4}$}

The hot repository branches are all paths beginning with an explicit interaction of the descending flow with the thermal output of the repository. In the first case (two-phase convection with condensation cap, Paths $\widehat{14.1}$ and 14.2), leakage from the fractures to the matrix pores feeds the unsaturated/saturated flow system to supply water to the condensation cap (Sketch G6). The additional fluid may locally depress the condensation cap toward the waste containers and possibly reach some containers. This interaction is likely to be episodic because storms that feed runoff are episodic. During this convective process, evaporation and condensation will leave behind precipitates, which we expect to alter hydraulic parameters of the rock. After the temperature of the repository has dropped enough that liquid water can reach containers, there will be return flow from the condensation cap. This reflux could be as saturated flow, Path $\widehat{14.1}$ (Sketch J3, p. 64), or as unsaturated flow, Path $\widehat{14.2}$ (Sketch J4, p. 66). For the path with saturated reflux, the remainder of the scenario is like Path $\widehat{12.1}$. Path $\widehat{14.1}$ gives us the scenario described by Sketches C, A, A1, A2, N, G6, I, I1, J3, K4, and M3.

The alternative possibility for reflux is unsaturated return of fluid from the condensation cap, Path 14.2 (Sketch J4). The episodic unsaturated plume can occur at any time in the repository's history, even after the reflux has already begun. This plume is an additional fluid source that may cause saturated flow in the sense of the composite model. Whether Path 14.2 occurs is an issue for modeling and experiment. The remainder of the scenario is like Path 12.2. Path 14.2 gives us the scenario $\mathrm{C}, \mathrm{A}, \mathrm{A} 1, \mathrm{~A} 2, \mathrm{~N}, \mathrm{G} 6, \mathrm{I}, \mathrm{I} 1, \mathrm{~J} 4, \mathrm{~K} 5$, and M1.

The second case for the hot repository interacting with the descending flow is two-phase convection with venting, Path $\widehat{14.3}$. Here there is a dryout zone around the repository. Any water reaching this zone evaporates and vents to the surface. Interception of episodic runoff, which is conveyed by fractures into the Topopah Spring units and leaks into the unsaturated flow system (Sketch G7), sees the same fate unless it overwhelms the dryout zone locally by reason of volume (Sketch G8). Continuing evaporation alters hydraulic parameters by plugging pores and fractures with precipitates (Sketch I1, p. 36). After containers have cooled sufficiently for water to exist at the containers, there is unsaturated reflux from surrounding rock-probably most rapidly as water vapor. In addition, the episodic unsaturated flow from runoff can now reach the waste containers (Sketch J5, p. 48) and mobilize contaminants (Sketch K5, p. 68). Since mobilization is by and large in an unsaturated flow system, there must be physical contact between rock and container for mobilization to occur. Contaminants move to the Calico Hills units and on to the water table in a primarily unsaturated flow system (Sketch M1). Path $\widehat{14.3}$ gives us the scenario C, A, A1, A2, N, G7, G8, I1, J5, K5, and M1.

The last hot-repository path, Path $\widehat{14.4}$, involves two-phase convection with formation of a heat-pipe regime (Sketch G9). For some period, there will be a dryout zone extending into the rock around a container. The influx of fluid in the unsaturated flow plume leaking from fractures is superimposed on the heat pipe. This acts as an additional source of fluid and as a temperature sink, cooling the condensation end of the heat pipe, possibly depressing its location. For low-enough temperature or high-enough unsaturated flux, the unsaturated plume could 


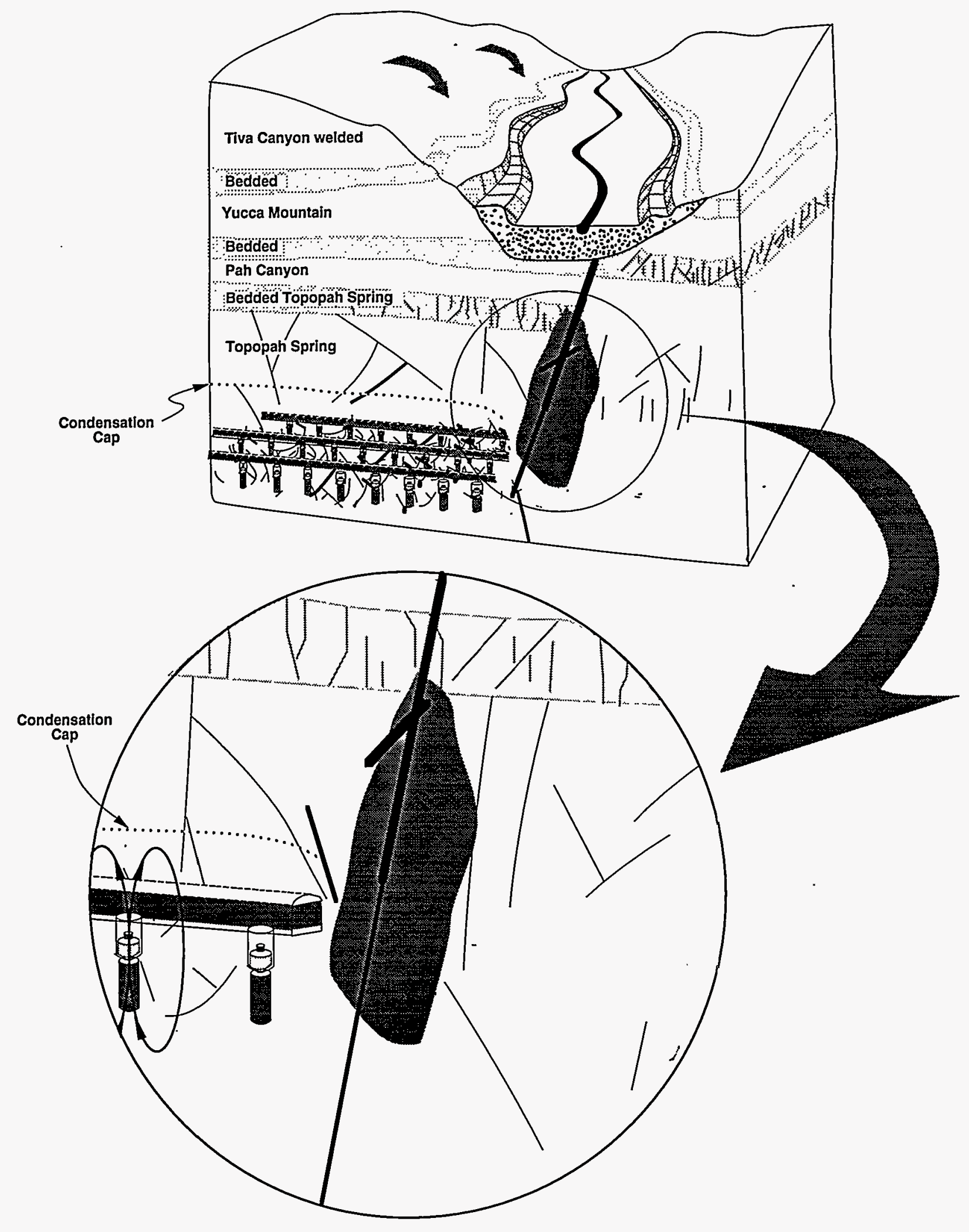

Sketch G6. Unsaturated-flow plume moving through the stress-altered region to reach waste containers and interacting with the condensation cap. 

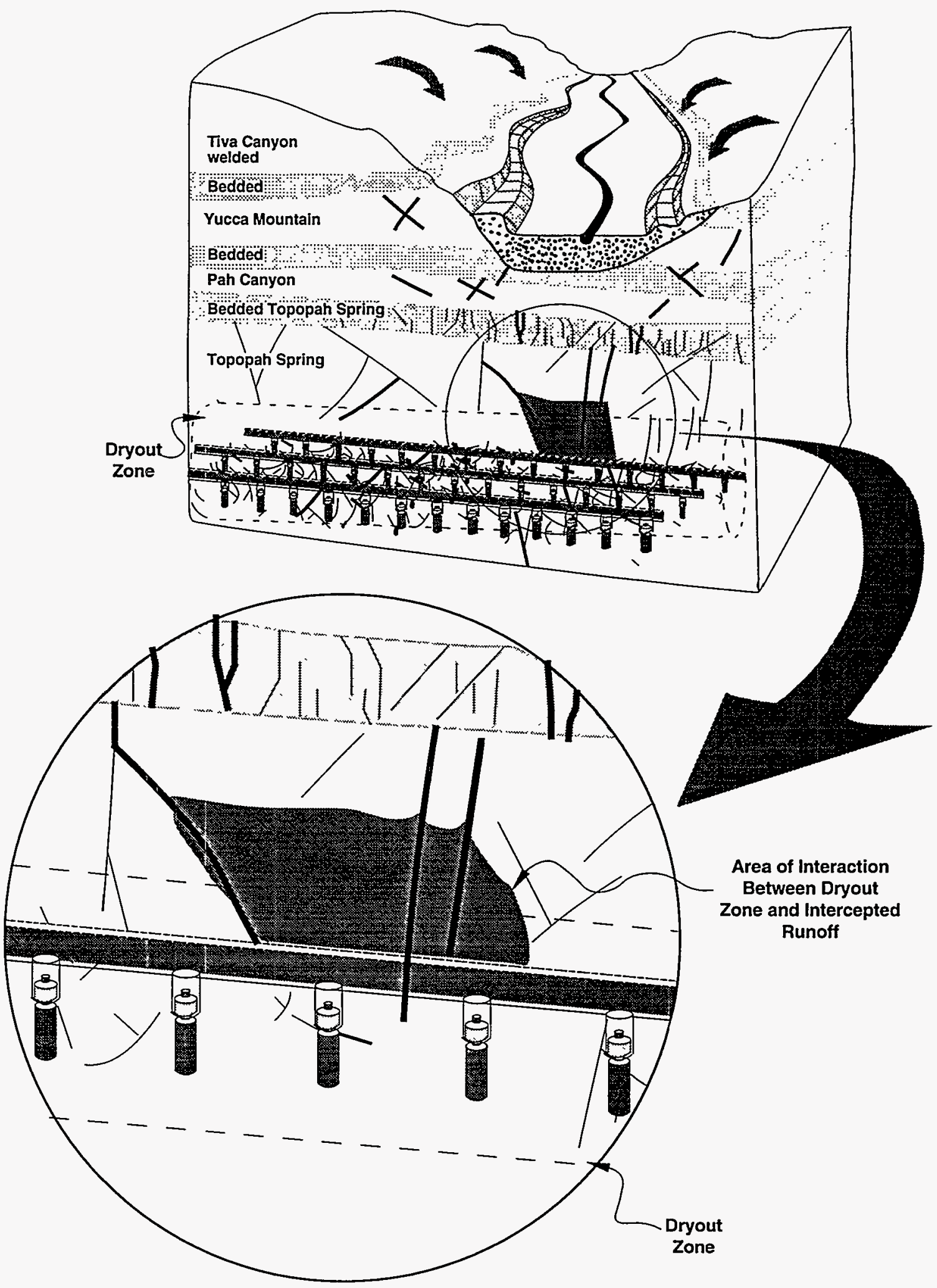

Sketch G7. Intercepted runoff carried by saturated fractures to the dryout zone. 

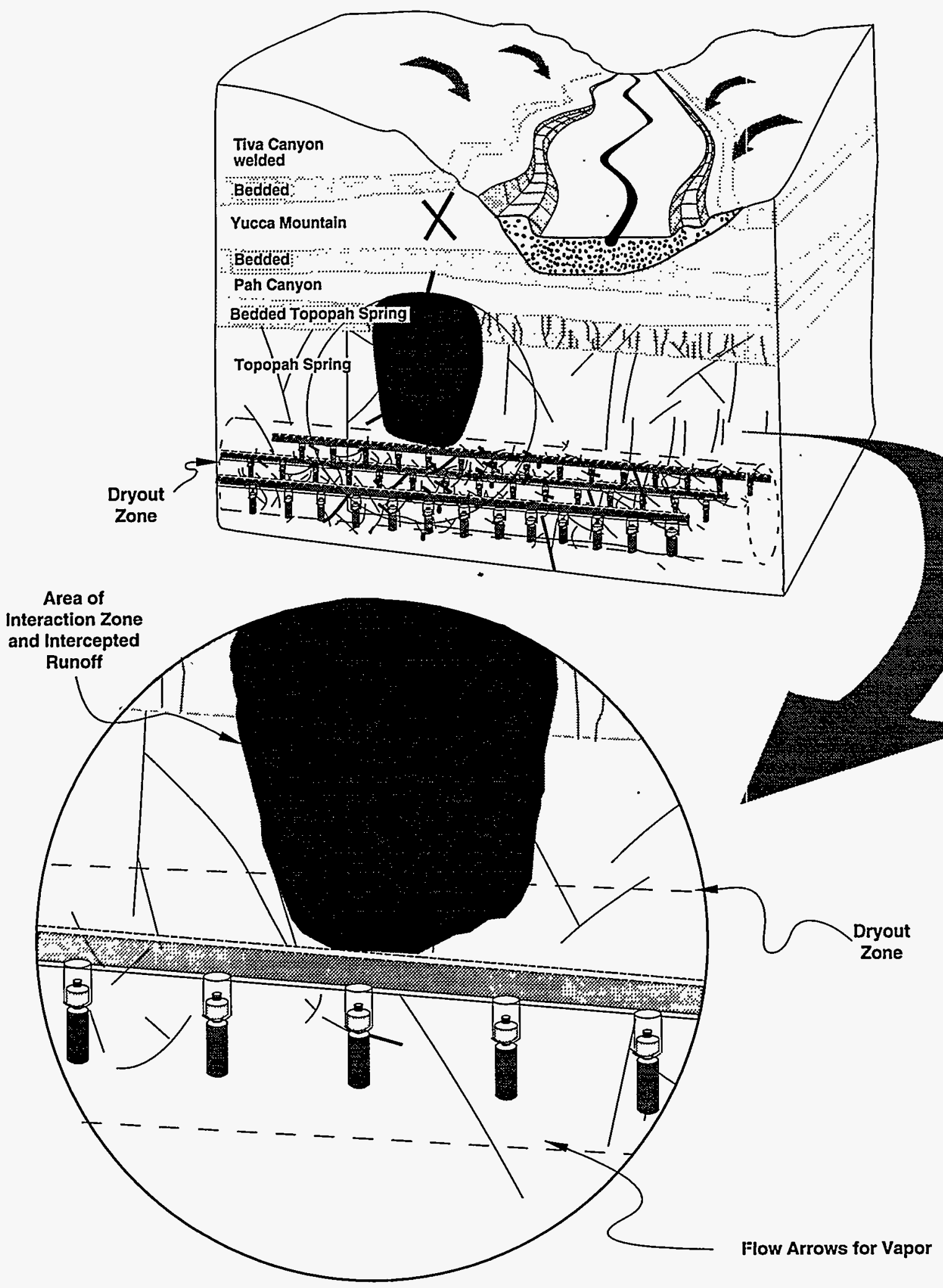

Sketch G8. Intercepted runoff forming an unsaturated flow plume that interacts with the dryout zone. 


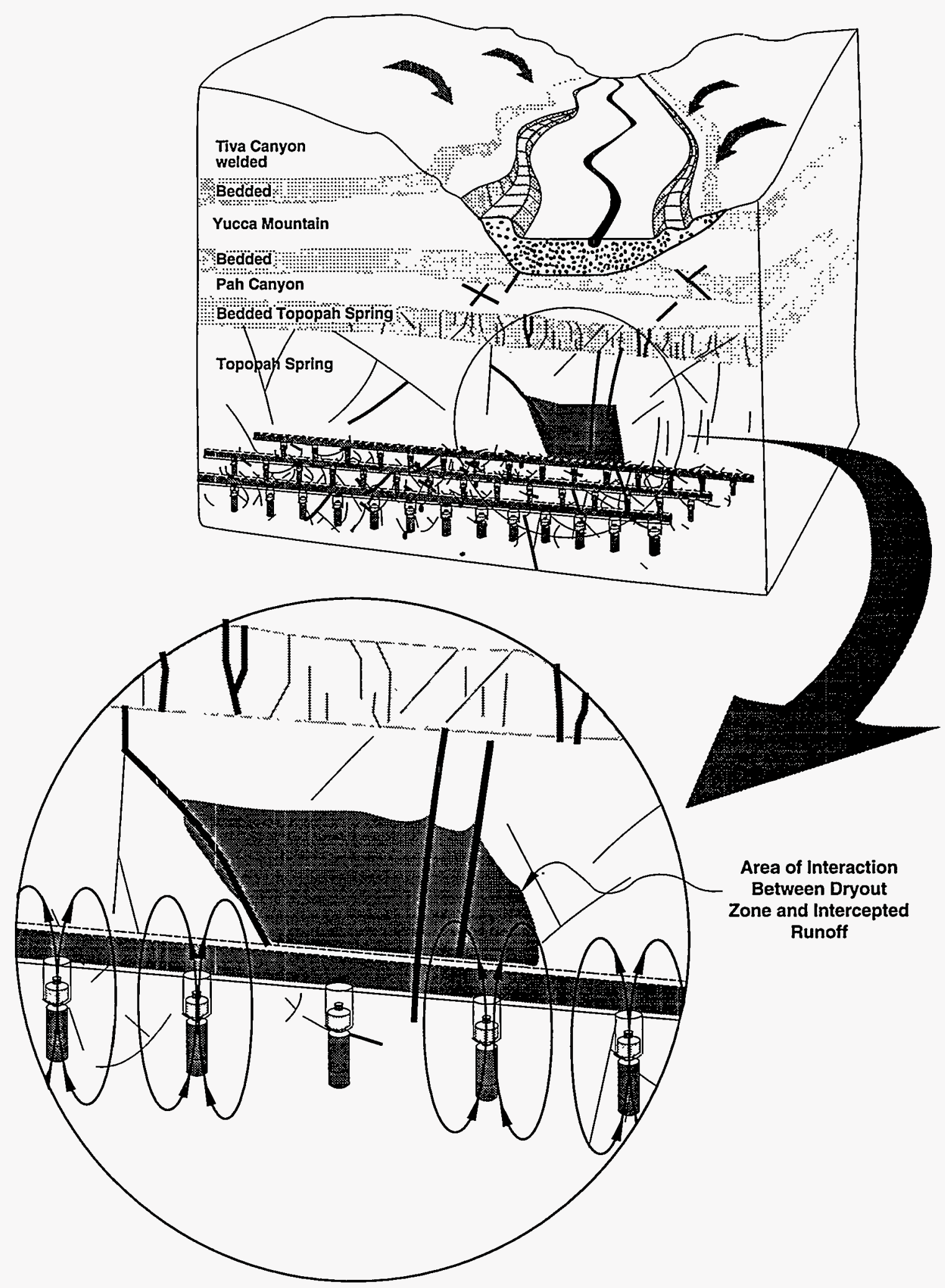

Sketch G9. Interaction of intercepted runoff that forms an unsaturated flow plume with the heat-pipe regime. 


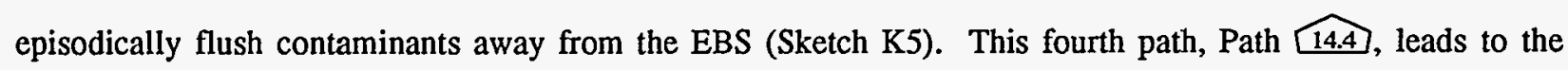
construction of the scenario defined by Sketches $C, A, A 1, A 2, N, G 9, I 1, ?, K 5$, and $M 2$.

\subsubsection{Cold Repository, Path $\widehat{14.5}$}

Most of the life of the repository is at a temperature below vaporization-the cold repository mode. This corresponds to Path 14.5. Flow down fractures to the Topopah Spring units that is imbibed by the matrix reaches the stress-altered region and must make its way around the maze of new fractures to the repository (Sketch G11). The fact that we are considering unsaturated flow makes a transition back to fracture flow in the same medium unlikely (unless there is some mechanism to focus flow to the region around fractures). The degradation of the EBS that occurs after that caused by the hot repository occurs in unsaturated mode. The episodic unsaturated plume adds to the unsaturated flow system reestablished when the repository cooled and flushes contaminants in an unsaturated flow front. Path 14.5 encompasses the scenario described by Sketches C, A, A1, A2, N, G11, K5, and M1.

\subsubsection{Flow Into Shallow Fractures and Direct Infiltration}

The above discussion of flow intercepted by exposed strata focused on fracture flow to the Topopah Spring units. Two other possible paths are represented in the tree: interception by shallow fractures and infiltration directly into the rock matrix (Sketch A7). The former path describes intercepting fractures that are much shallower than the Topopah Spring units but which capture runoff and feed it into the mountain. Any further movement of this captured runoff involves the same processes discussed under fracture flow to the Topopah Spring units. The latter path, direct infiltration, describes the imbibition of runoff into the matrix. This direct infiltration, along with surface soil infiltration, includes the possibility of local saturation at or near the surface that at some depth changes to unsaturated flow. Processes involved have also been discussed under the case of fracture flow to the Topopah Spring units. The paths are shown in Tree Segments 15 (p. 132) and 16 for both processes. Basically the arguments are the same as for fracture flow to the Topopah Spring units with the essential difference that there is no rapid movement of fluid down fractures to the depth of the Topopah Spring units. All percolation processes begin close to the surface and involve the detailed hydraulic behavior of the numerous stratigraphic units between the surface and the repository. We will therefore not discuss the paths in this tree segment in detail because the FEPs were discussed earlier. Changed cartoons are indicated on the tree segment. The absence of discussion and detail does not mean that the indicated paths are unimportant: experiment and calculation may in fact show that this tree segment describes a major component of infiltration into Yucca Mountain.

$$
\begin{aligned}
& \text { C, A, A1, A7, A3, A4, I, J, K, K1, M, M1 } \\
& \text { C, A, A1, A7, A3, A4, I, J1, K2, M1 } \\
& \text { C, A, A1, A7, A3, A8, I1, A6, K2, M1 }
\end{aligned}
$$

Path 15.1

Path 15.2

Path 15.3 


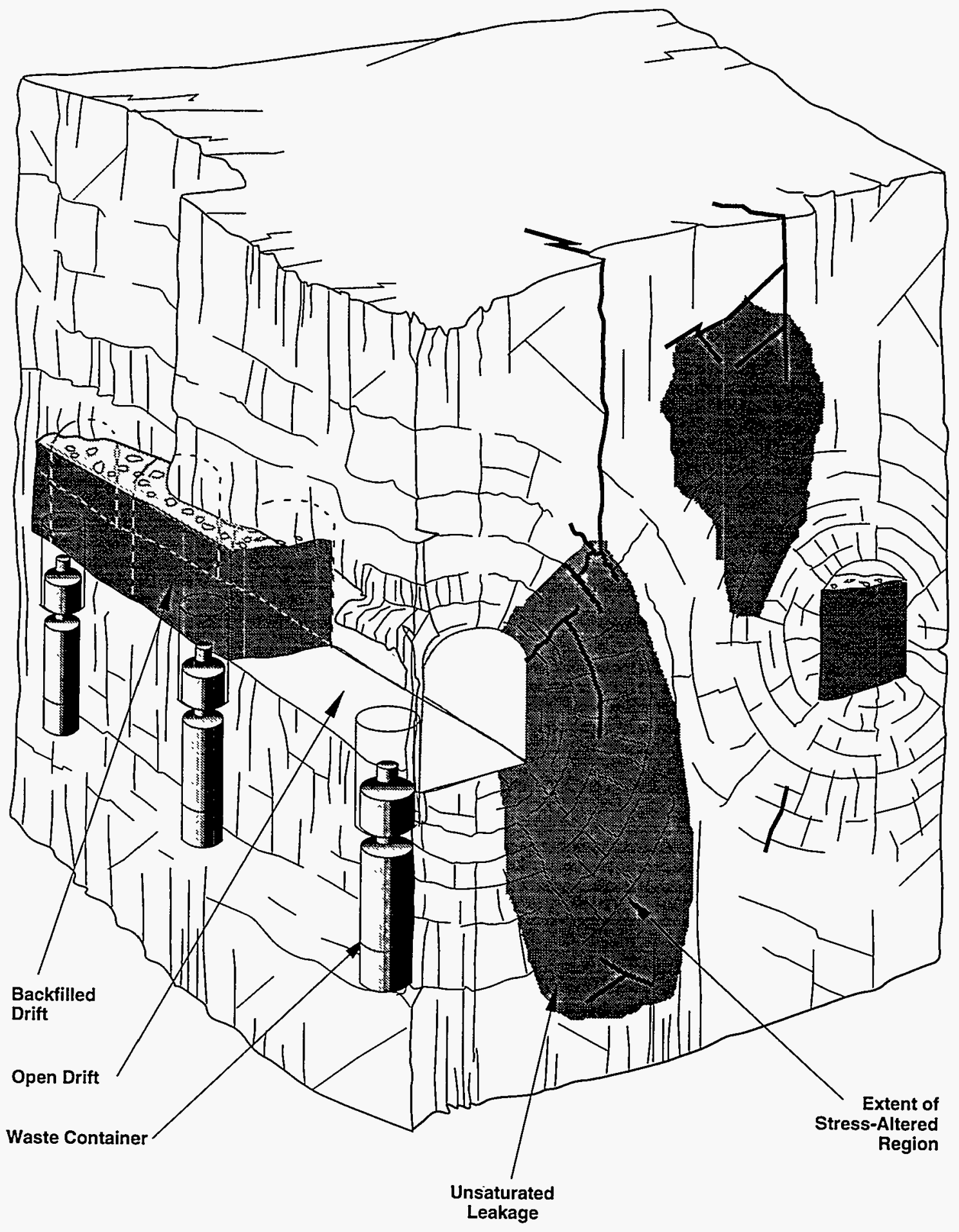

Sketch G11. Enhanced unsaturated flow plume reaching the stress-altered region. 


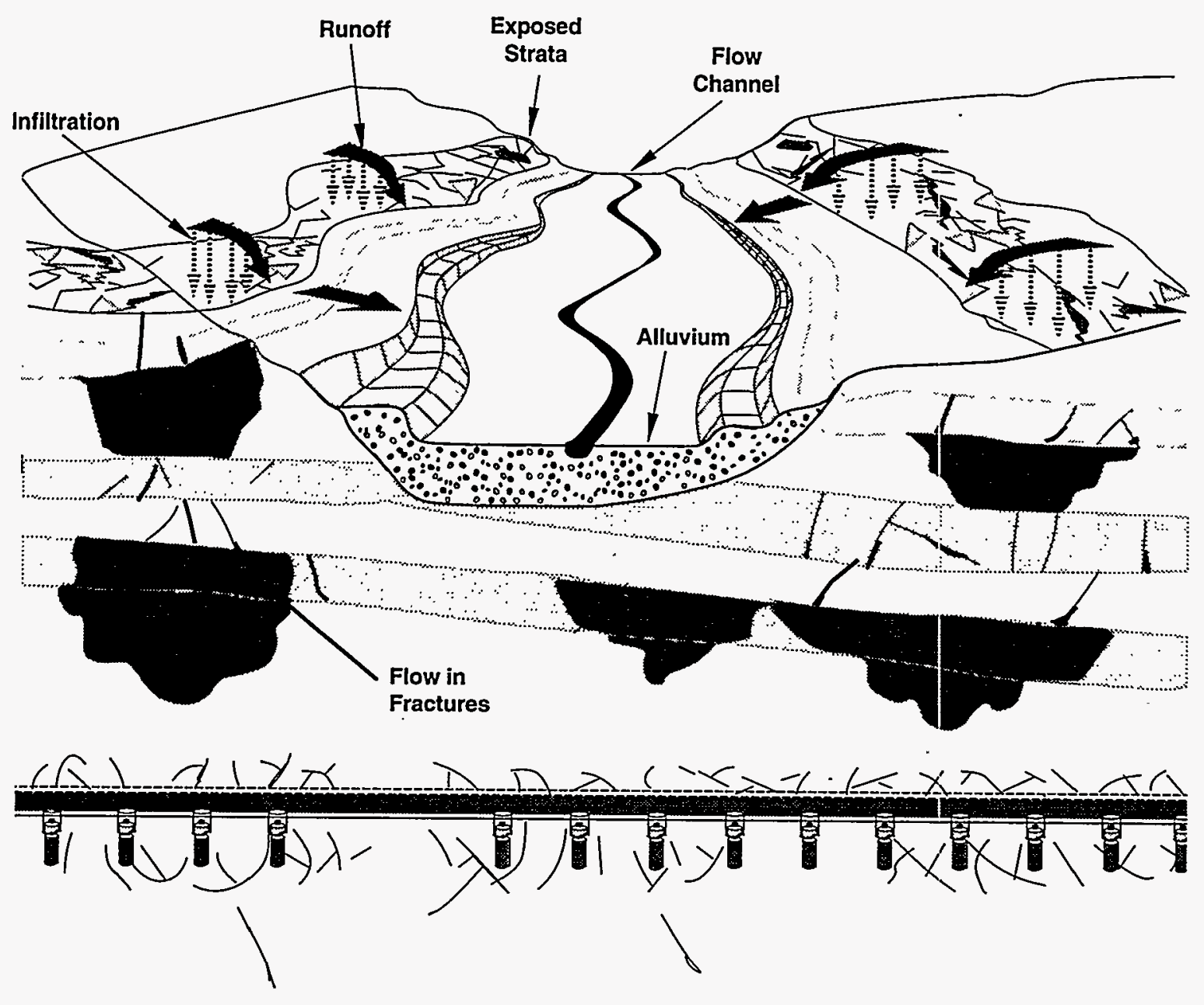

Sketch A7. Intercepted runoff being converted to unsaturated flow. 


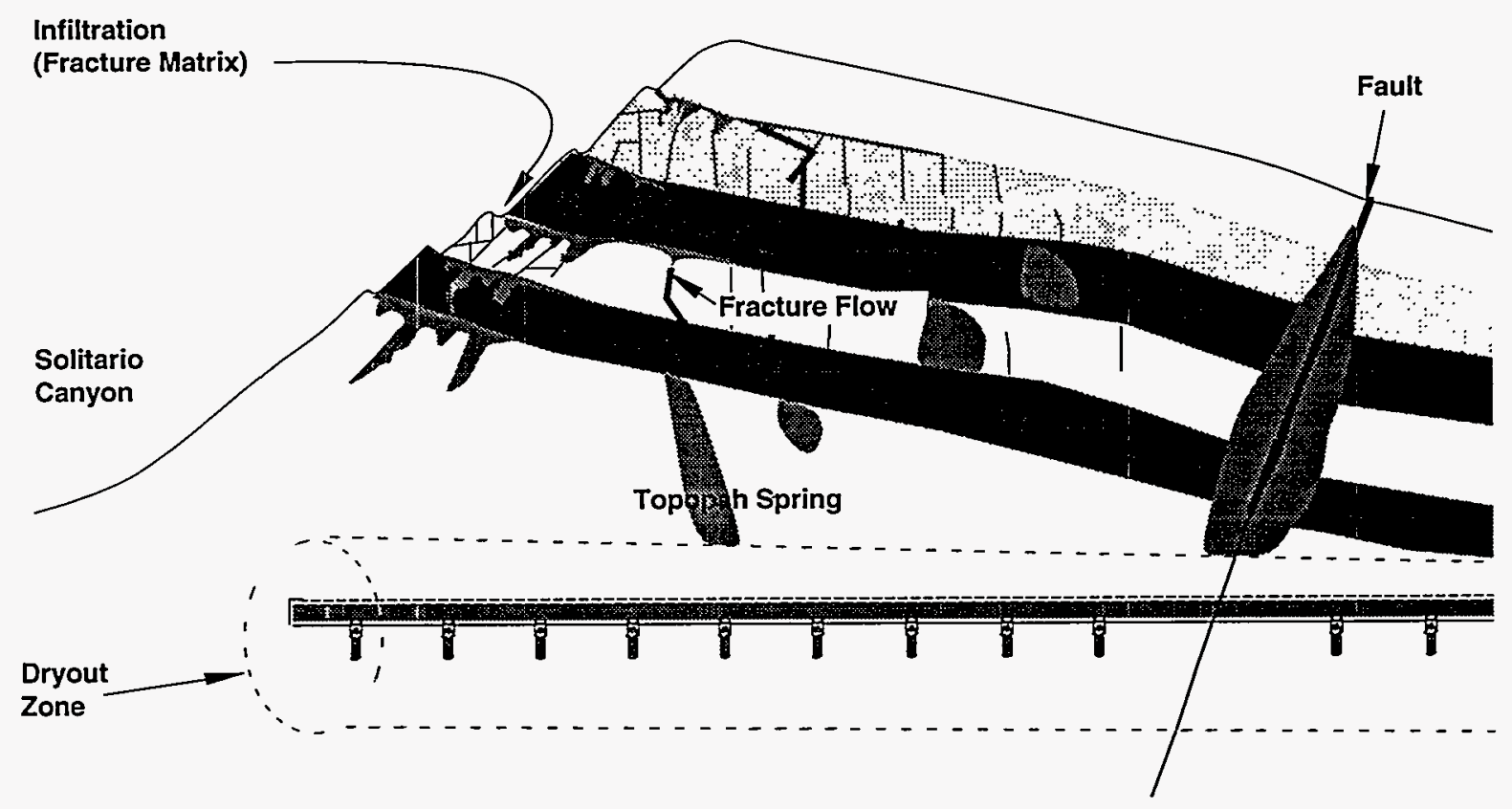

Sketch A8. Distributed infiltration reaching the dryout zone. 
C, A, A1, A7, A3, H2, I2, K2, M2

Path 15.4

C, A, A1, A7, A3, G11, K5, M

Path 15.5

C, A, A1, A7, N, G13, I, I1, J3, K4, M, M1

Path 16.1

C, A, A1, A7, N, G13, I, I1, J3, K5, M1

Path 16.2

C, A, A1, A7, N, G7, I1, K5, M1

Path 16.3

C, A, A1, A7, N, G9, I1, K5, M2

Path 16.4

C, A, A1, A7, N, G5, K5, M

Path 16.5

This concludes the discussion of intercepted runoff. 


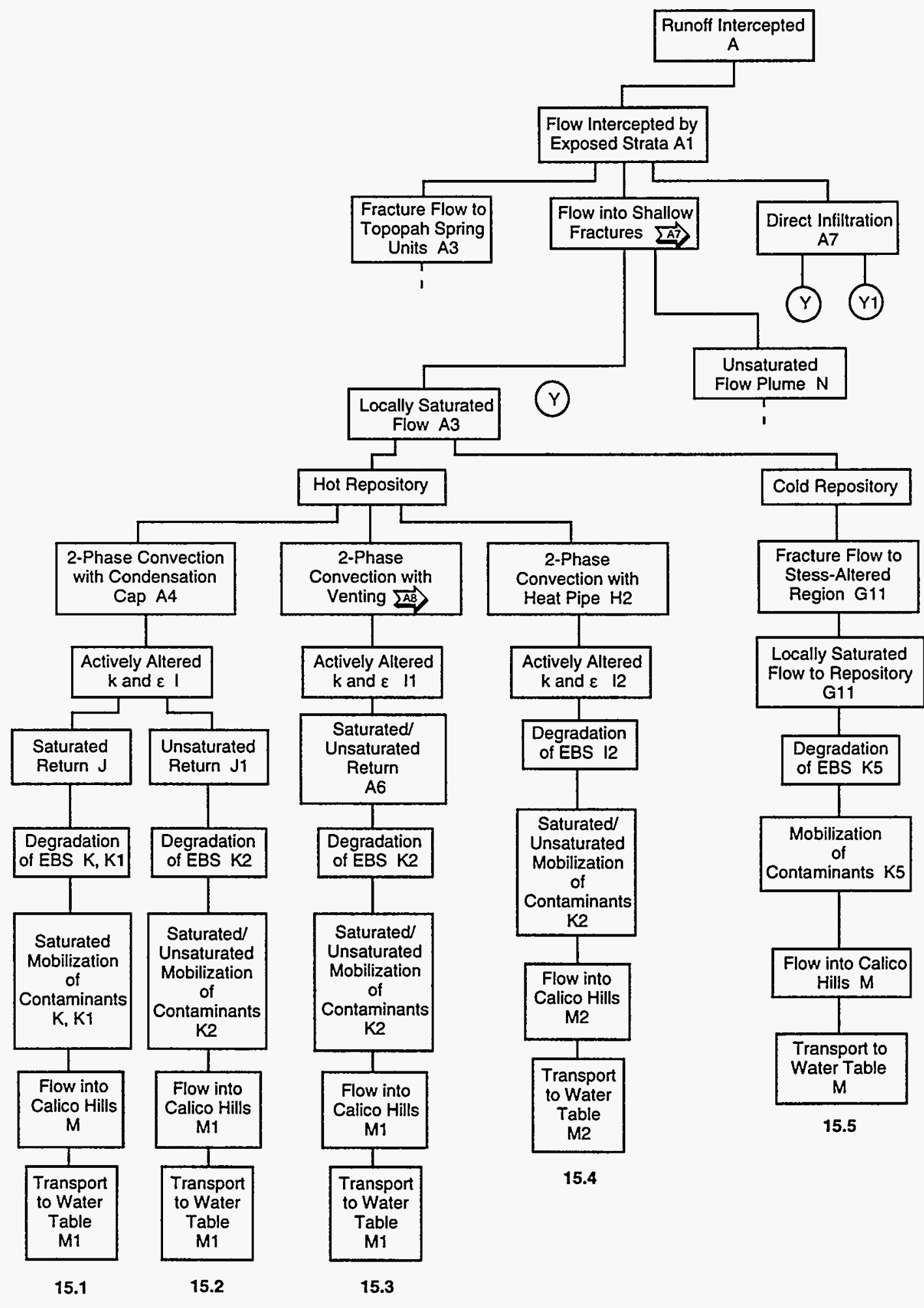

a. Events and their relationships, with cross-references to sketches illustrating each event.

Tree Segment 15. Nominal Flow, Runoff-Producing Events, Runoff Intercepted, Flow Intercepted by Exposed Strata, Flow into Shallow Fractures, Locally Saturated Flow. 


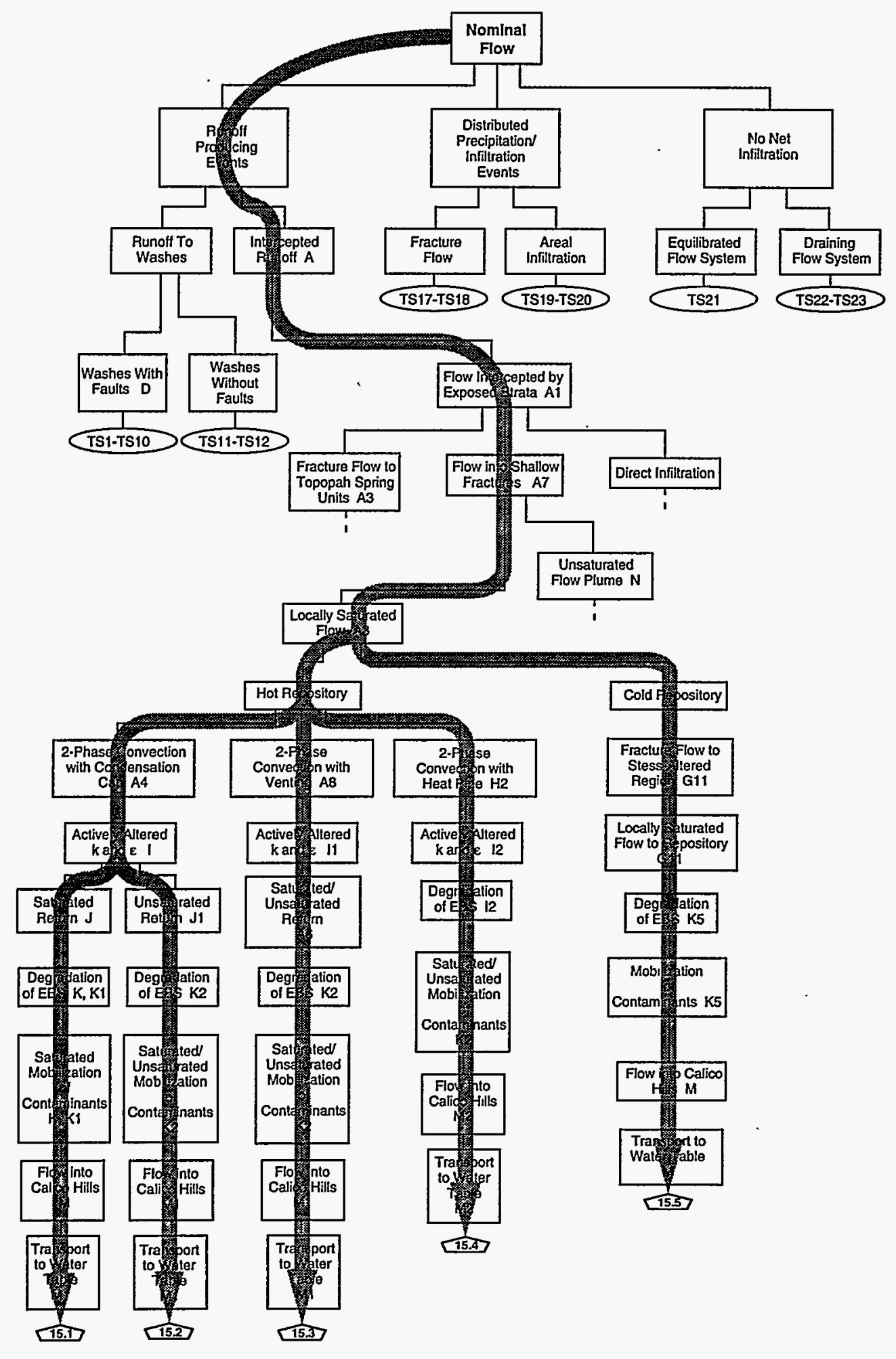

b. Scenario pathways, showing the relationship of the tree segment to the overall event tree for nominal flow. 


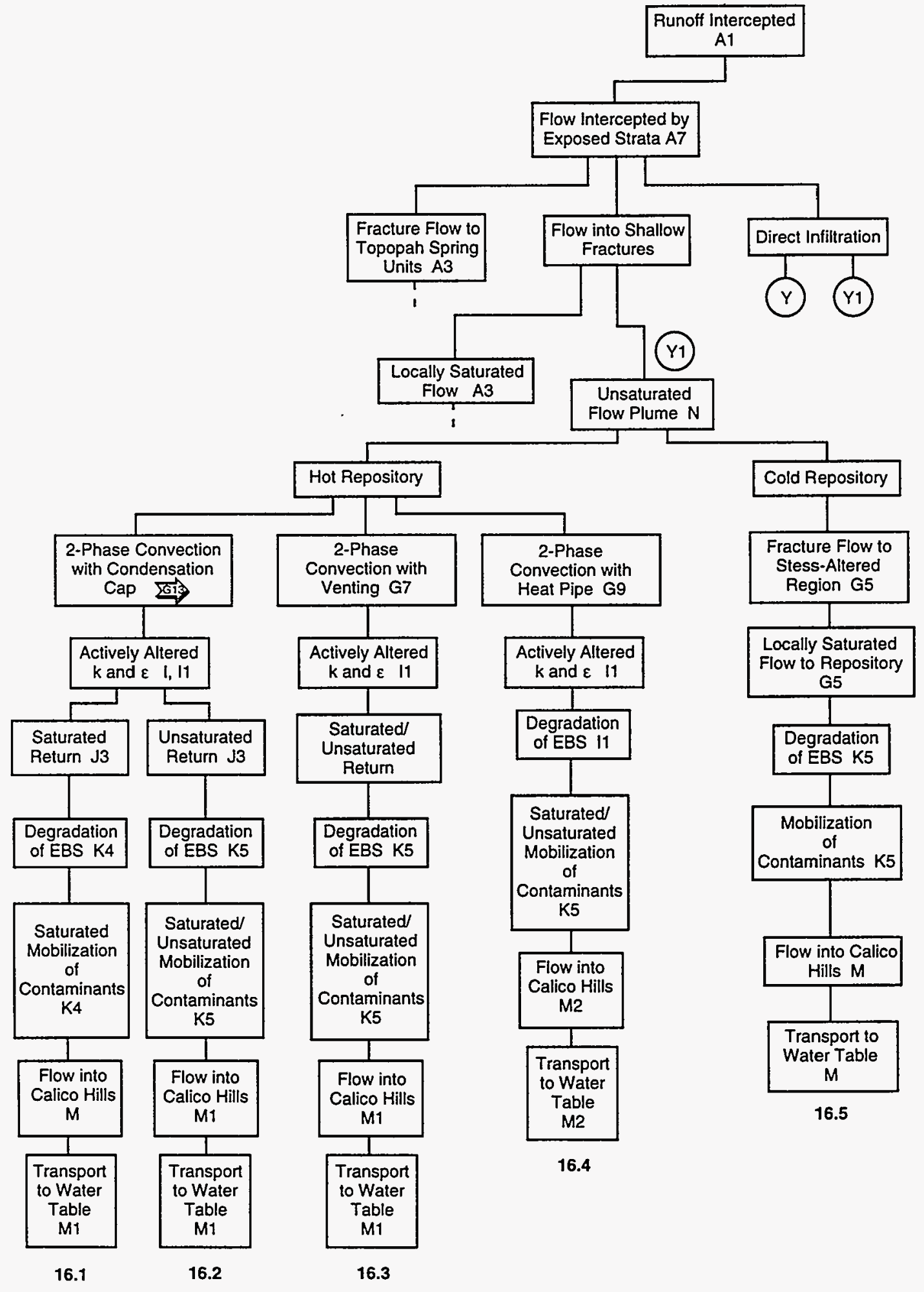

a. Events and their relationships, with cross-references to sketches illustrating each event.

Tree Segment 16. Nominal Flow, Runoff-Producing Events, Runoff Intercepted, Flow Intercepted by Exposed Strata, Flow into Shallow Fractures, Unsaturated Flow Plume. 


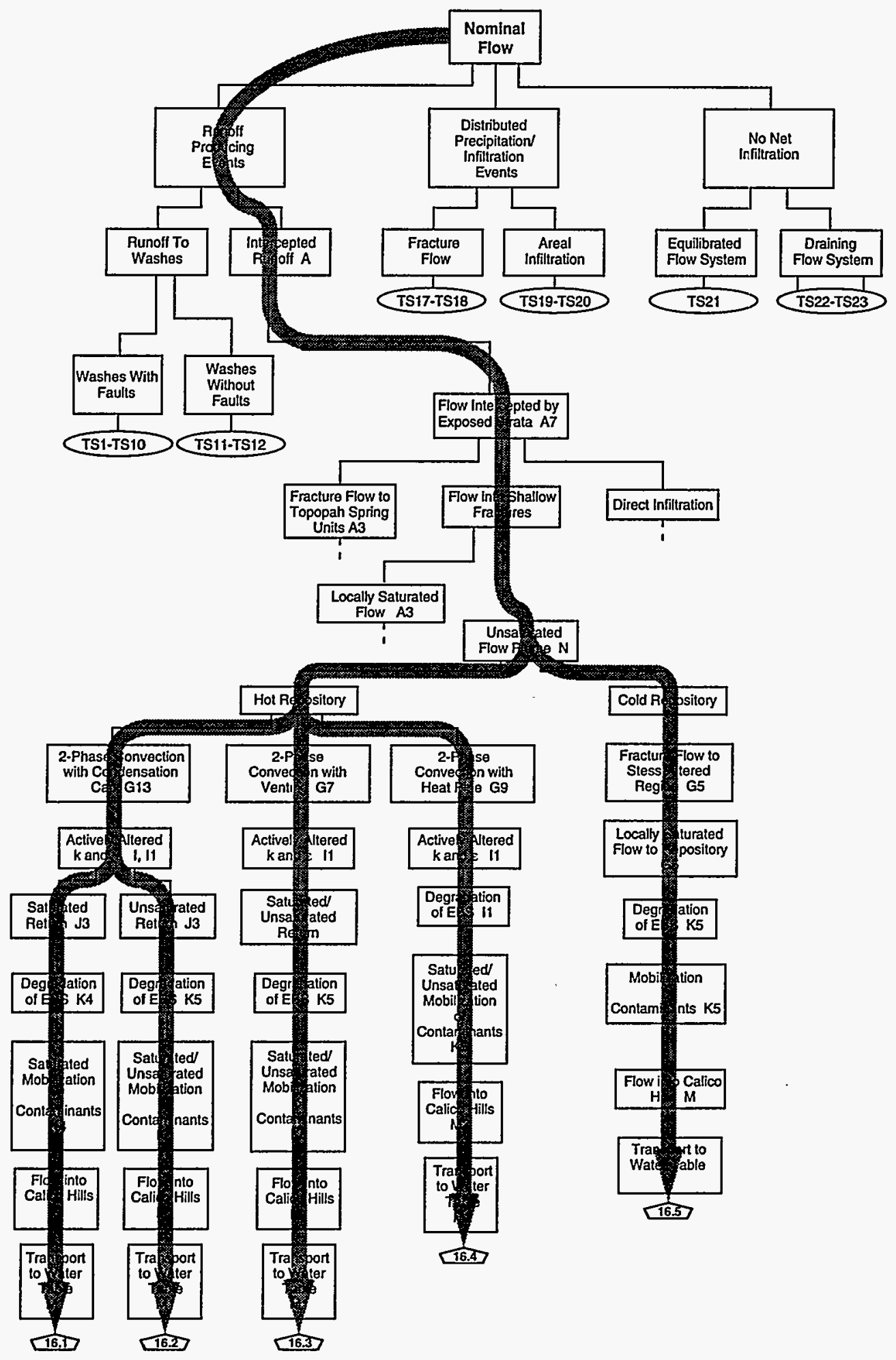

b. Scenario pathways, showing the relationship of the tree segment to the overall event tree for nominal flow. 


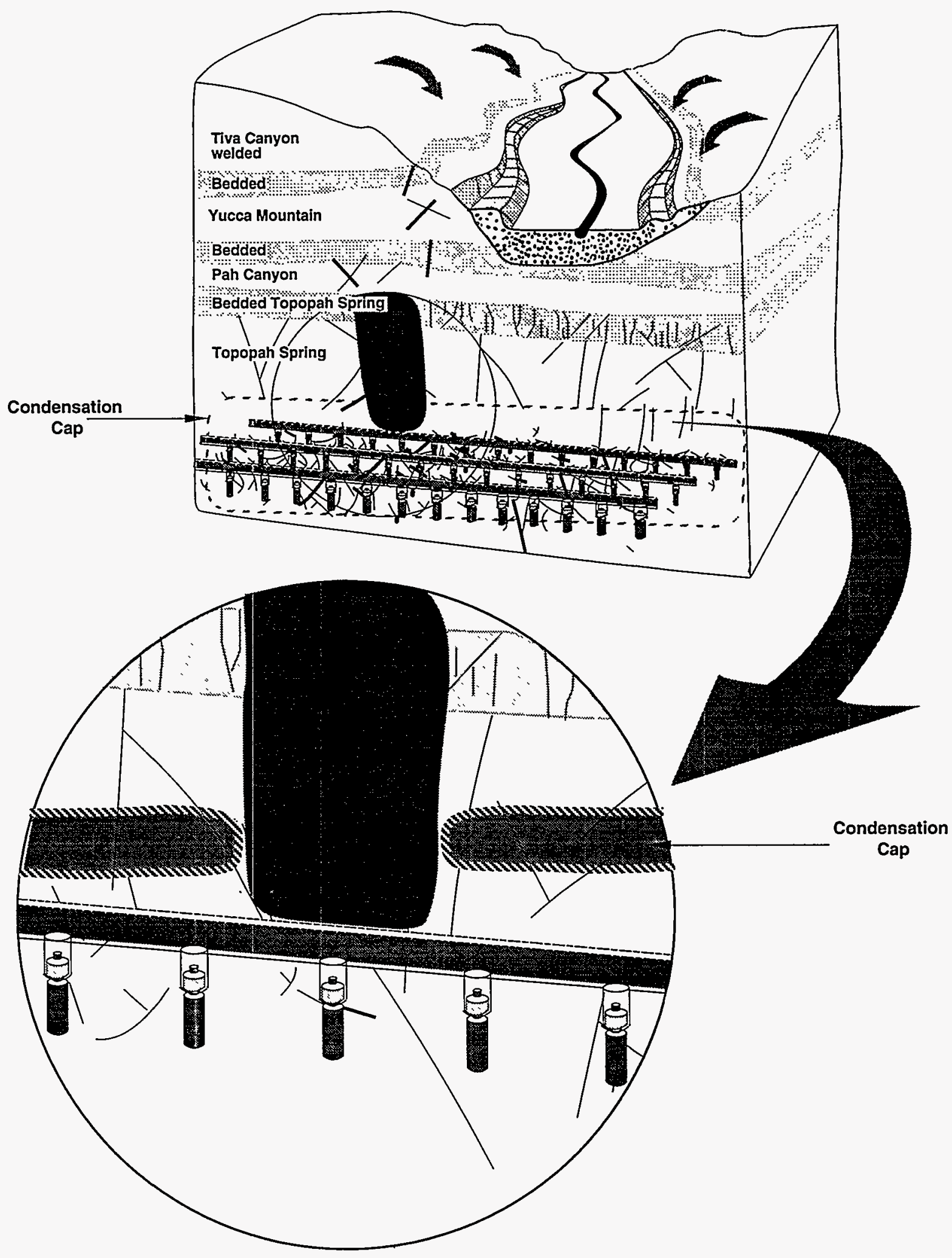

Sketch G13. Intercepted runoff forming an unsaturated plume that interacts with the condensation cap. 


\section{DISTRIBUTED PRECIPITATION AND INFILTRIATION}

The middle branch in Figure 3 (p. 16), "Distributed Precipitation/Infiltration Events," will now be discussed. For any precipitation event, soil and rocks receiving the moisture have some capacity for infiltration. Distributed infiltration (Sketch B) is differentiated into two fates for the precipitation-fracture flow, that is, capture of precipitation by exposed fractures, and areal infiltration, that is, capture of precipitation directly into the pores of soil and rock. The two branches reflect different rates of penetration past the first few meters from the surface and presumably different responses to evapotranspiration.

\subsection{Fracture Flow}

\subsubsection{Shallow Fracture Flow (Imbibition to Matrix)}

Starting with fracture flow, we first construct scenarios involving shallow fractures that terminate, allowing imbibition into the matrix. These scenarios begin with distributed precipitation and infiltration, suggested by Sketch B. Shallow fractures open to the surface catch the fluid (Sketch B1). Water in such a shallow system may evaporate after a storm has passed, but to affect the mountain at depth, precipitation must penetrate more than a few tens of centimeters and be sorbed by the rock matrix. The mountain is a more or less layered structure, the separate tuffaceous strata having differing hydraulic properties. The contrasts between welded and nonwelded units play an essential role in local saturation, lateral diversion, and formation of an unsaturated flow plume. The basic ideas behind the complex hydraulic behavior of rocks above the repository are shown in Sketch B2 (p. 142). This sketch shows episodic infiltration superimposed on the background of fluids already in the mountain. The unsaturated/ saturated plumes shown in some strata represent the load added to this background after some precipitation event. Presumably there is also a general downward flux of fluid in the mountain. Most of the various units above repository level are unsaturated. Whether the mountain is draining, integrating and smoothing current infiltration, or static with only vapor adjustments is a question we hope can be answered during site characterization.

\subsubsection{Hot Repository, Paths Through 17.4}

After fluid has penetrated to the Topopah Spring units (and perhaps sooner), it reaches the region of influence of the hot repository. In Path $\widehat{17.1}$ of Tree Segment 17, two-phase convection is accompanied by formation of a condensation cap (Sketch A4, p. 117). The location of the cap and degree of participation of the water table depends on the thermal output of the repository. In Path 17.2. the reflux from the condensation cap is an unsaturated/saturated return (composite model) (Sketch J4); in this case, backfill in the drift could participate in the flow system. The remainder of the two scenarios are like Paths $\widehat{12.1}$ and $\widehat{12.2}$. The scenario produced by Path 


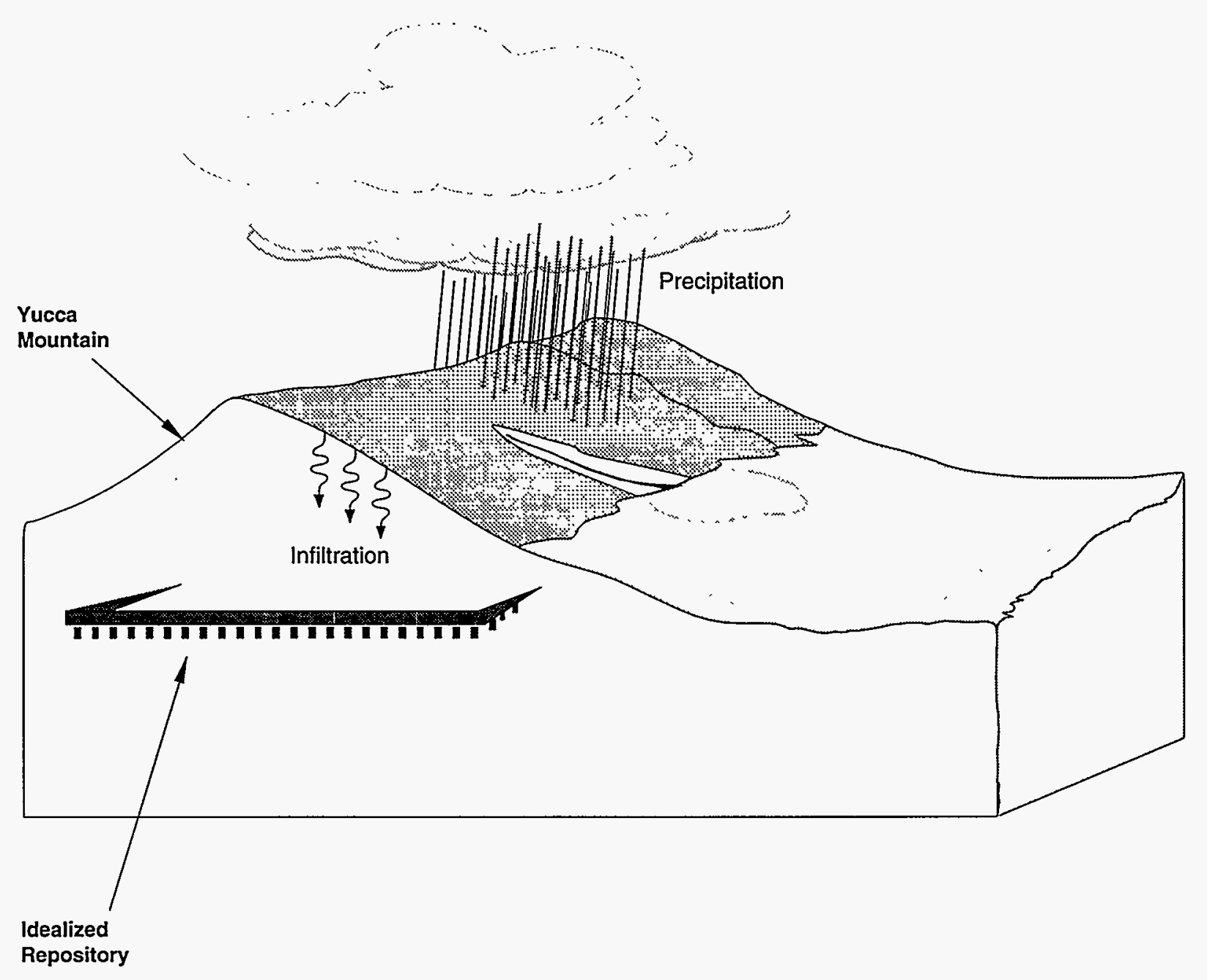

Sketch B. Distributed precipitation and infiltration. 


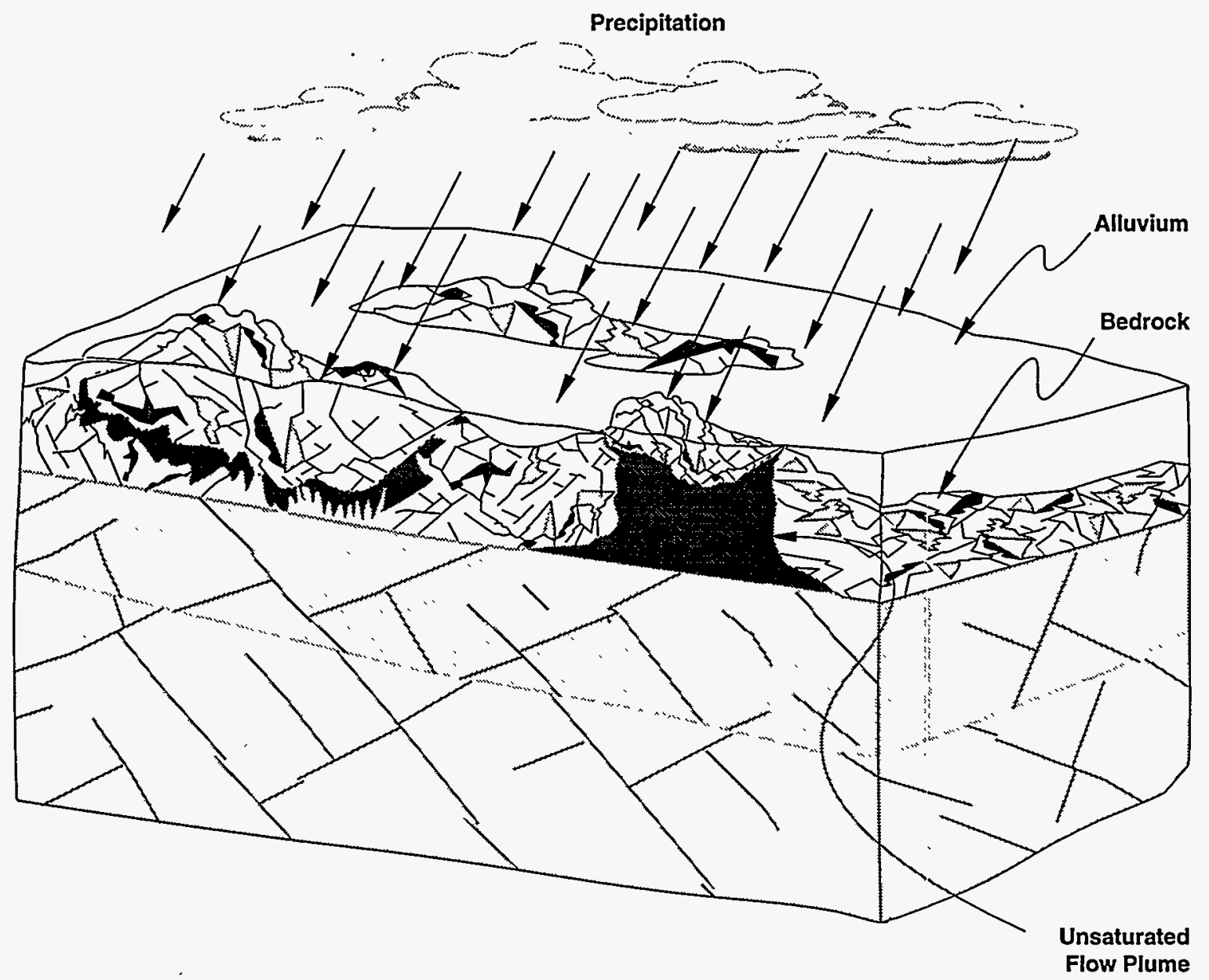

Sketch B1. Detail of surface distribution of rocks and soil, showing some infiltration. 


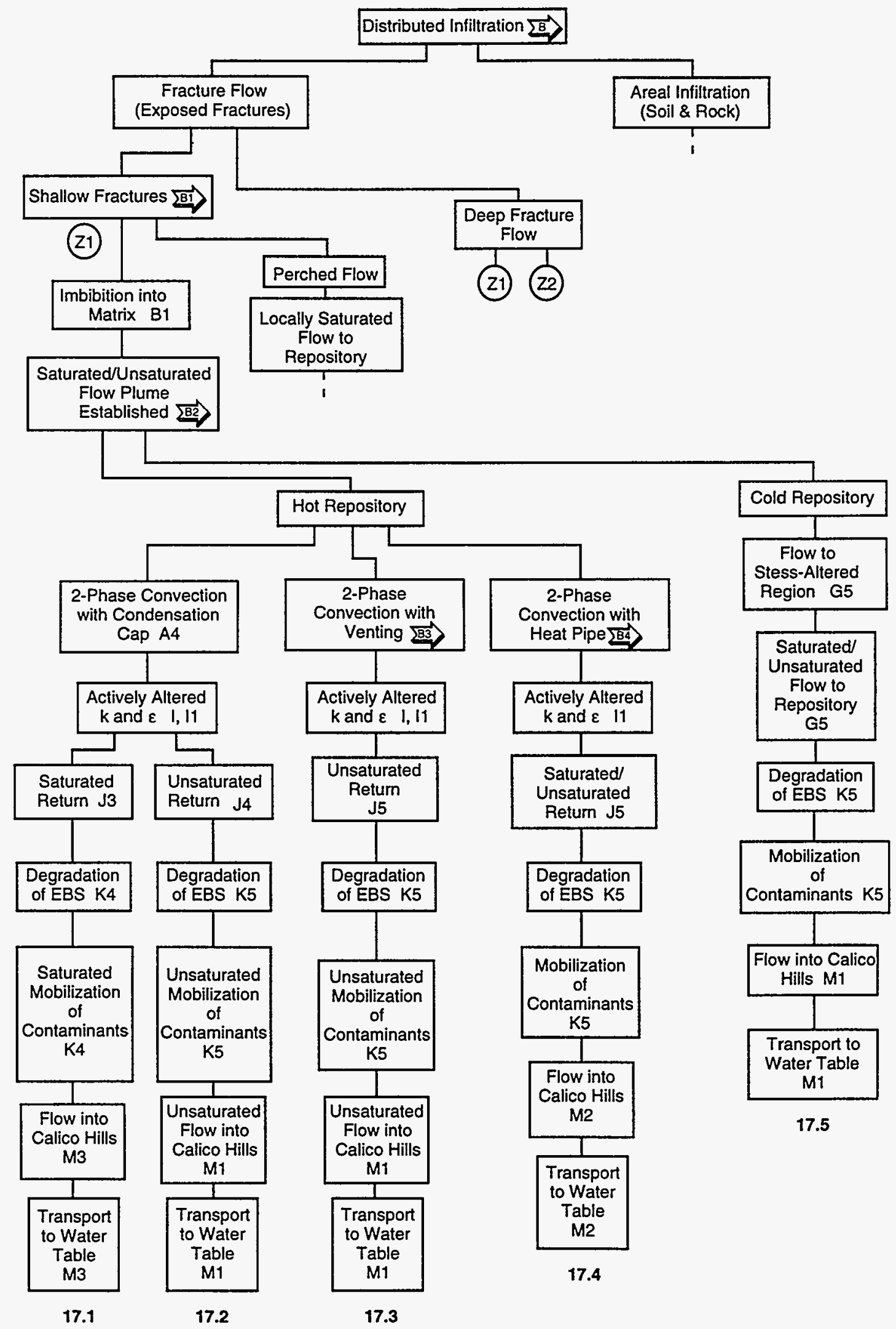

a. Events and their relationships, with cross-references to sketches illustrating each event.

Tree Segment 17. Nominal Flow, Distributed Infiltration, Fracture Flow, Shallow Fractures, Imbibition to Matrix. 


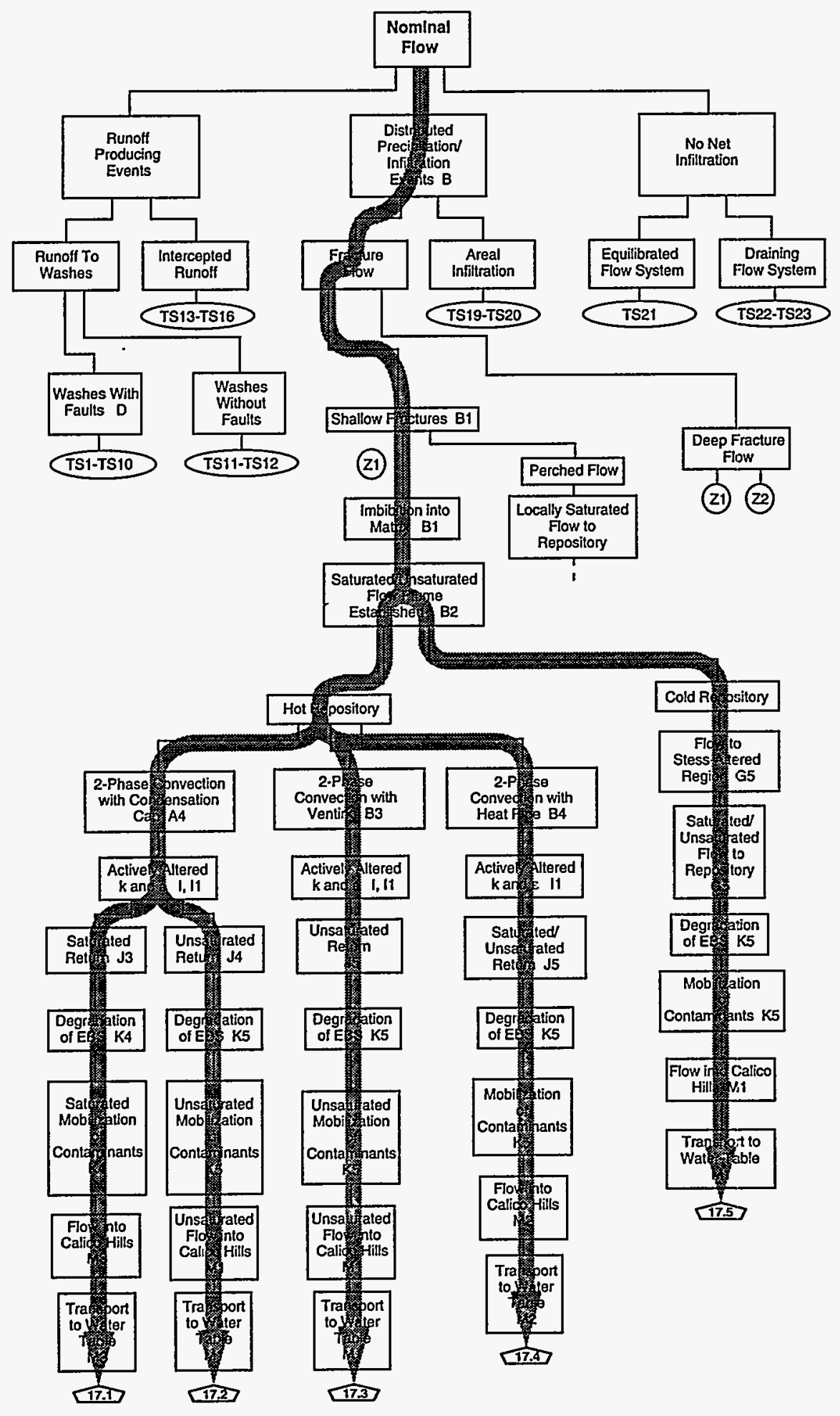

b. Scenario pathways, showing the relationship of the tree segment to the overall event tree for nominal flow. 


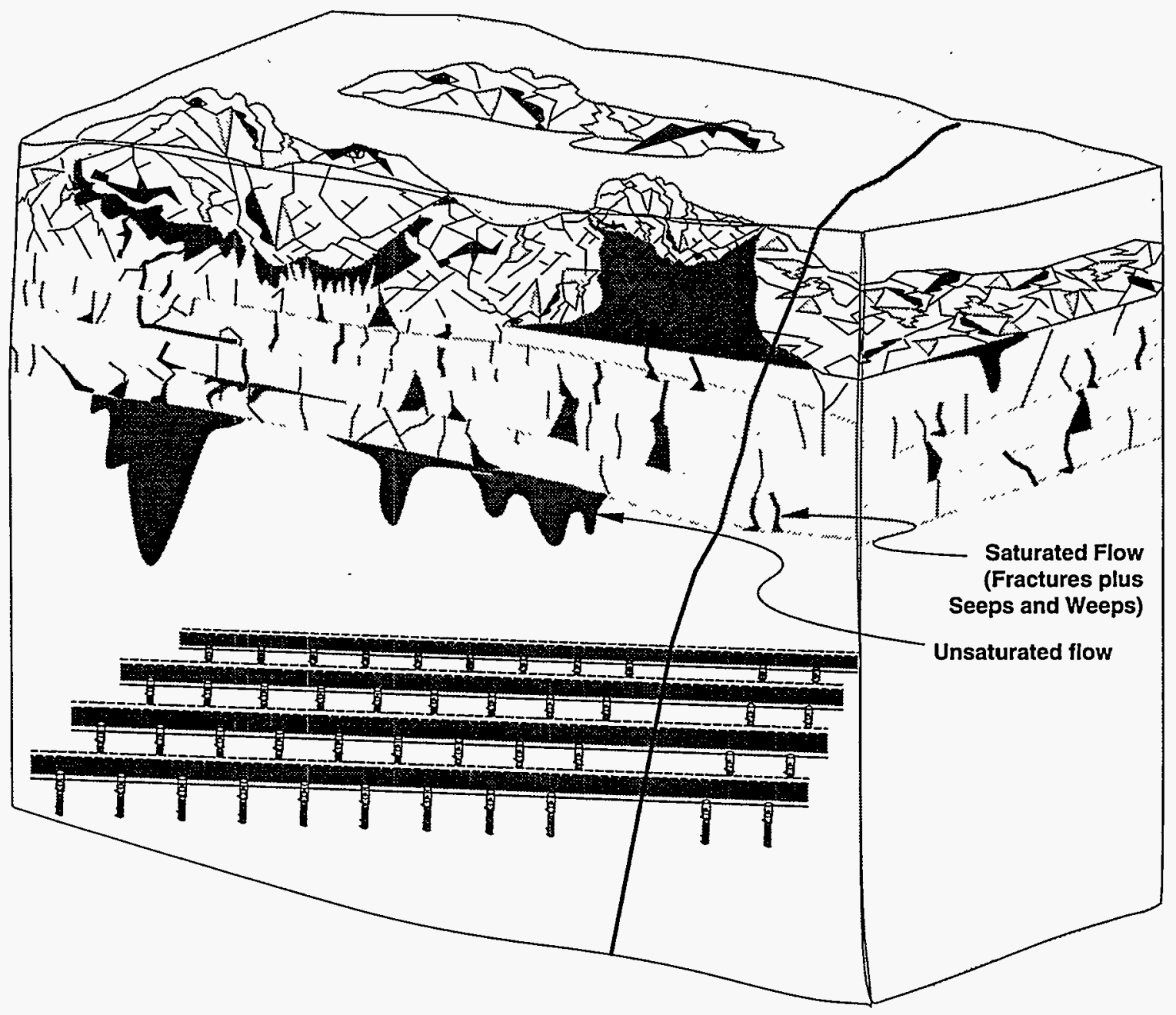

Sketch B2. Surface distribution of infiltration as affected by lithology. 
17.1 is described by Sketches B, B1, B2, A4, I, I1, J3, K4, and M3. Path 17.2 leads to the scenario described by Sketches B, B1, B2, A4, I, I1, J4, K5, and M1.

Path $\widehat{17.3}$ involves two-phase flow with venting of the water vapor to the atmosphere and a dryout zone around the waste containers. Sketch B3 shows the dryout zone surrounding the drifts. The location of this zone depends on the thermal output and the local density of packing of containers. The location of the vaporization isotherm changes as the thermal load changes: it may extend through drifts or it may be disconnected around containers. The remainder of the scenario is like Path $\widehat{14.3}$. Path 17.3 leads to the scenario described by Sketches B, B1, B2, B3, I, I1, J5, K5, and M1.

Path $\widehat{17.4}$ for a hot repository allows for development of a two-phase convective flow system in a heat pipe. The size of the heat pipe depends on thermal loading and on the elapsed time since container emplacement. Sketch B4 illustrates formation of heat pipes around each container, with condensation between containers. This state can occur soon after emplacement; however, for loadings currently under consideration, within a few years the heat from the containers may be sufficient to prevent return flow between containers along a single drift. Return flow could still continue in the rock between drifts; thus, the heat pipe would effectively be a linear source. When cooling is sufficiently advanced, if resaturation of the dryout zone has occurred, heat pipes around individual waste containers may be reestablished. In addition, containers will not have identical outputs, and thermal output may vary considerably along a drift (Ryder, 1993). Therefore the size, shape, and time of formation of the pipes will be variable. Path $\widehat{17.4}$ continues with the alteration of the hydraulic parameters in roughly the zone experiencing evaporation. We say roughly because mineralogical alterations are expected since solubilities are temperature dependent. Sketch II is a stand-in, showing the location rather than processes. In order for non-gaseous contaminants to move, liquids must partially resaturate the dryout zone and reach the waste containers. Fluids for such resaturation come from surrounding cooler rock (by capillary forces), from vapor transport, and from the infiltration-driven percolation that originated this discussion. Sketch J5 shows percolation in the form of an unsaturated flow plume originating from episodic precipitation on the mountain. Degradation of the EBS, which may have begun with establishment of two-phase convective flow when steam first passed the waste container or even earlier, is reinvigorated by the return of liquids to the waste container. Calculations for various thermal loadings and orientations of waste containers indicate that the dryout period may be substantial (on the order of 1000 years) (Johnson, 1991; Johnstone and Gnirk, 1982; Johnstone et al., 1982; Ryder, 1993: Buscheck and Nitao, 1993). Once liquids compromise the container, mobilization of the contaminants can begin. Since the processes are located in an unsaturated flow field with possibly some locally saturated flow, mobilization occurs under the same conditions (Sketch K5). Flow carries contaminants into the Calico Hills nonwelded unit and on to the water table as shown in Sketch M2. Path 17.4 produces the scenario described by Sketches B, B1, B2, B4, I1, J5, K5, and M2. 


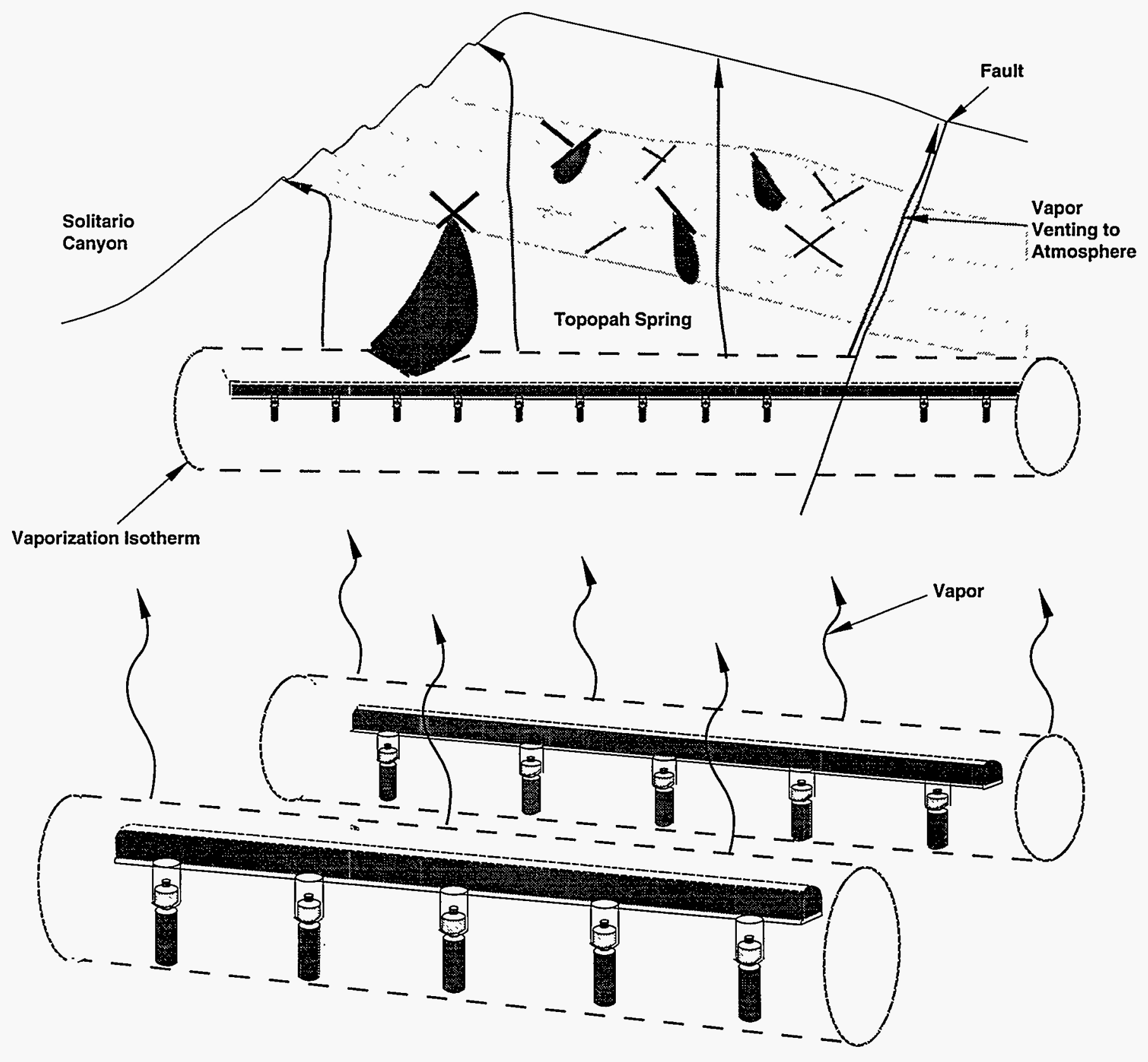

Sketch B3. Two-phase flow with venting of water vapor to the atmosphere. 

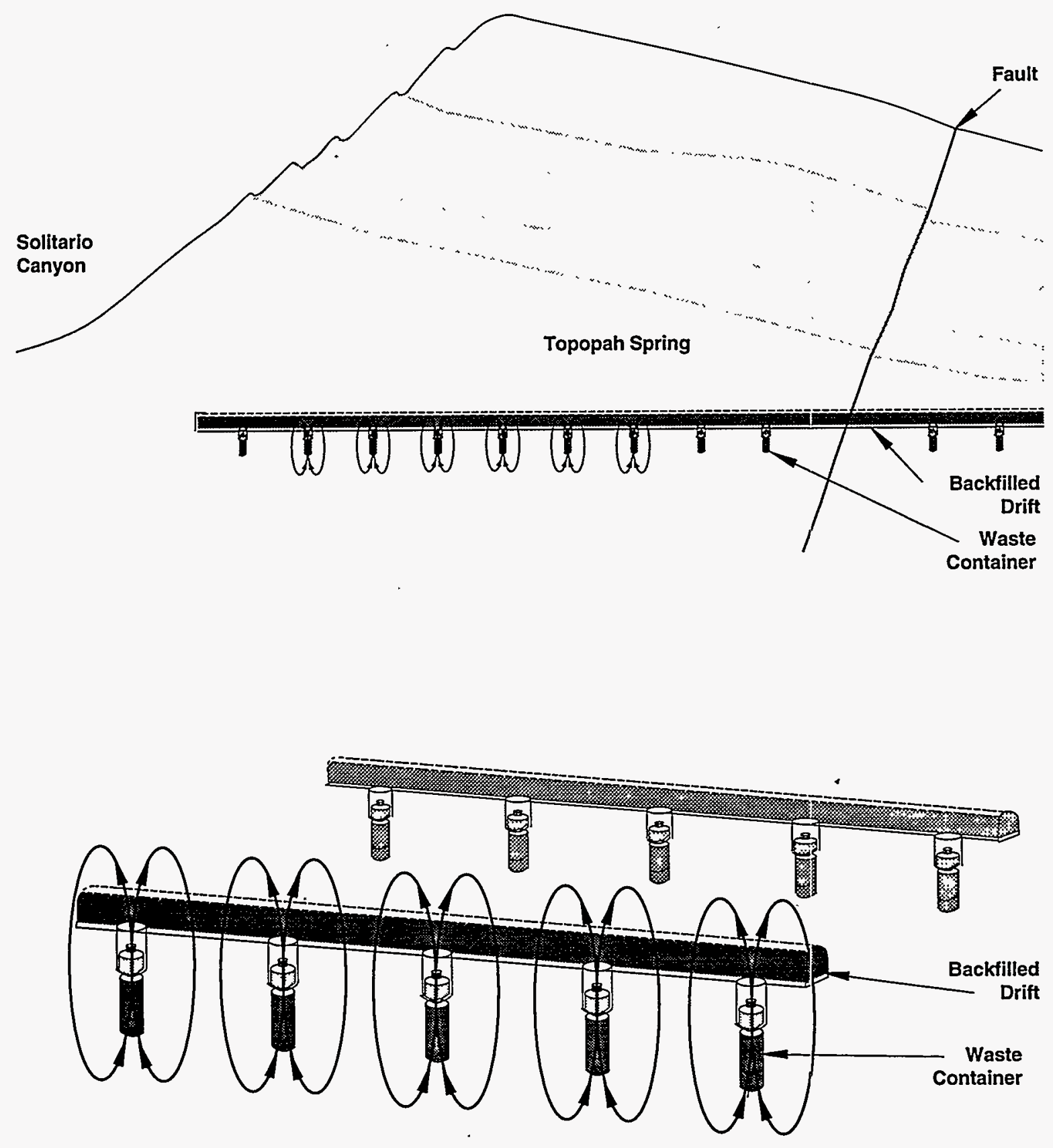

Sketch B4. General view of mountain, repository, and heat-pipe regime. 


\subsubsection{Cold Repository, Path $\widehat{17.5}$}

Path 17.5 addresses a cold repository. Fluids still arrive as an unsaturated/saturated flow plume (Sketch B2). New fractures in this region constrain the paths of unsaturated flow. Even in a cold repository, the waste container may be degraded by fluids resaturating the rock and reaching the container (Sketch G5). Both degradation and mobilization require some physical contact between rock and container (Sketch K5). Contaminants are carried into the Calico Hills units and on to the water table in an unsaturated flow field with some locally saturated flow (Sketch M1). Path $\widehat{17.5}$ gives us the scenario described in Sketches B, B1, B2, G5, K5, and M1.

\subsubsection{Shallow Fracture Flow (Perched-Water Flow)}

\subsubsection{Hot Repository, Paths 18.1 Through 18.4}

Shallow fractures could feed perched-water flow systems (Tree Segment 18, p. 148). The five possible paths in Tree Segment 18 are similar to the previous five in that the perched-water flow system is a source of fluid to feed the interaction with the repository. All these five paths are built on Sketches B1, A3, and B5. Sketch B5 shows a set of hypothetical connected and partially connected fractures that are paths for perched-water flow. The partially connected fractures depend on locally saturated flow along contacts between hydraulically contrasting strata to establish the connection between fractures. Path $\widehat{18.1}$ considers the interaction of the locally saturated (perched) flow with a hot repository that produces two-phase convection and a condensation cap (Sketch A4, p. 117). Path 18.2 begins with the repository-produced condensation cap but allows for an unsaturated reflux from the cap to the repository (Sketch J1, p. 42). The rest of Paths $\widehat{18.1}$ and $\widehat{18.2}$ are like Paths 1.1 and 1.2 . Path 18.1 constitutes a scenario described by Sketches B, B1, A3, B5, A4, I, I1, J, K, K1, and M. Path 18.2 maps a scenario described by Sketches B, B1, A3, B5, A4, I, I1, J1, K2, and M1.

Rather than form a condensation cap, water vapor may be vented through the mountain to the atmosphere. Path 18.3 presumes fluid reaches the repository by flow from a perched-water system. This fluid arrives at the dryout zone established around the repository, evaporating there or depressing the dryout zone back toward the heat sources. The dryout zone is essentially defined by the location of the vaporization isotherm (Sketch A5, p. 118). Vaporization leaves behind precipitates in pores and fractures (Sketch I1, p. 36). In this path the return flow, which occurs after the vaporization isotherm is inside the container, is a saturated/unsaturated return. The perched-water flow system provides fluid locally to waste containers and also supplies fluid to the unsaturated flow system resaturating in the surrounding rock (Sketch A6, p. 119). Capillary forces and vapor flow from cooler rock surrounding the repository may also contribute to resaturation. External degradation of the EBS began at emplacement, and although degradation may have been slowed or stopped during dryout, it is reactivated by arrival of fluids at the containers (Sketch K3, p. 59). Mobilization of contaminants occurs in response to both saturated and unsaturated flow (Sketch K3). Flow into the Calico Hills units and on to the water table is expected to revert to the 


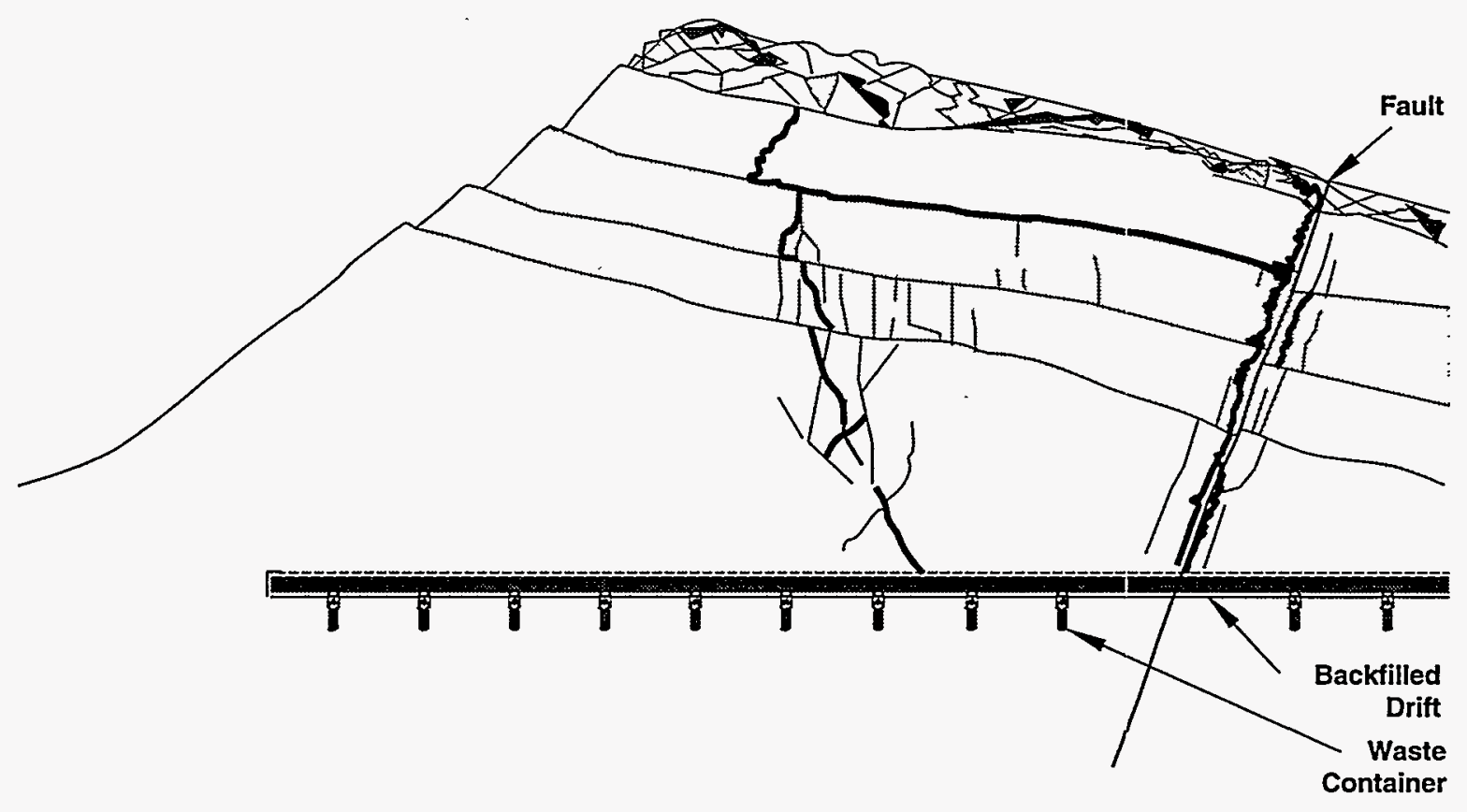

Sketch B5. Connected and partially connected fractures that are paths for perched flow to the repository. 


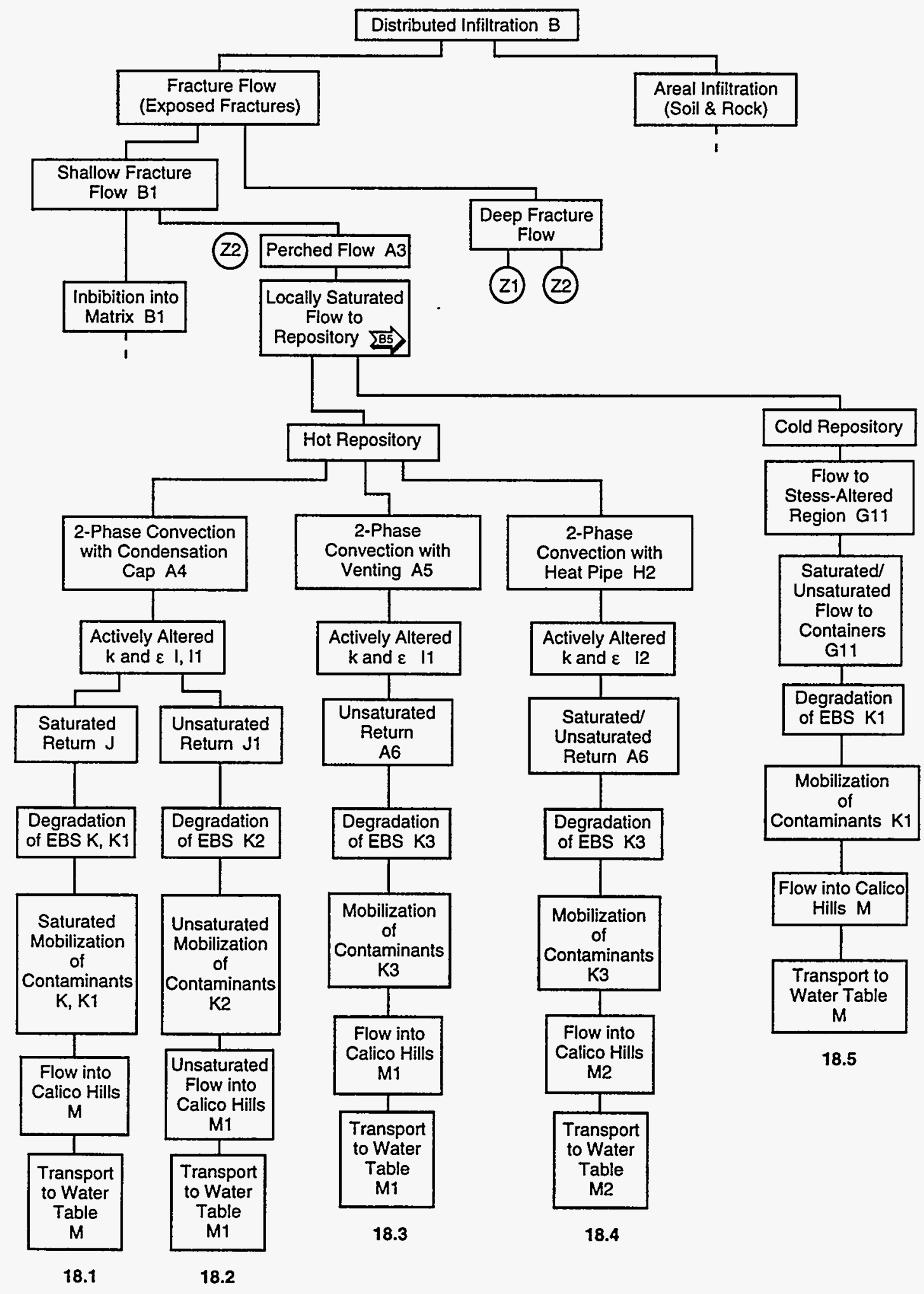

a. Events and their relationships, with cross-references to sketches illustrating each event.

Tree Segment 18. Nominal Flow, Distributed Infiltration, Fracture Flow, Shallow Fractures, Perched Flow, Locally Saturated Flow to Repository. 


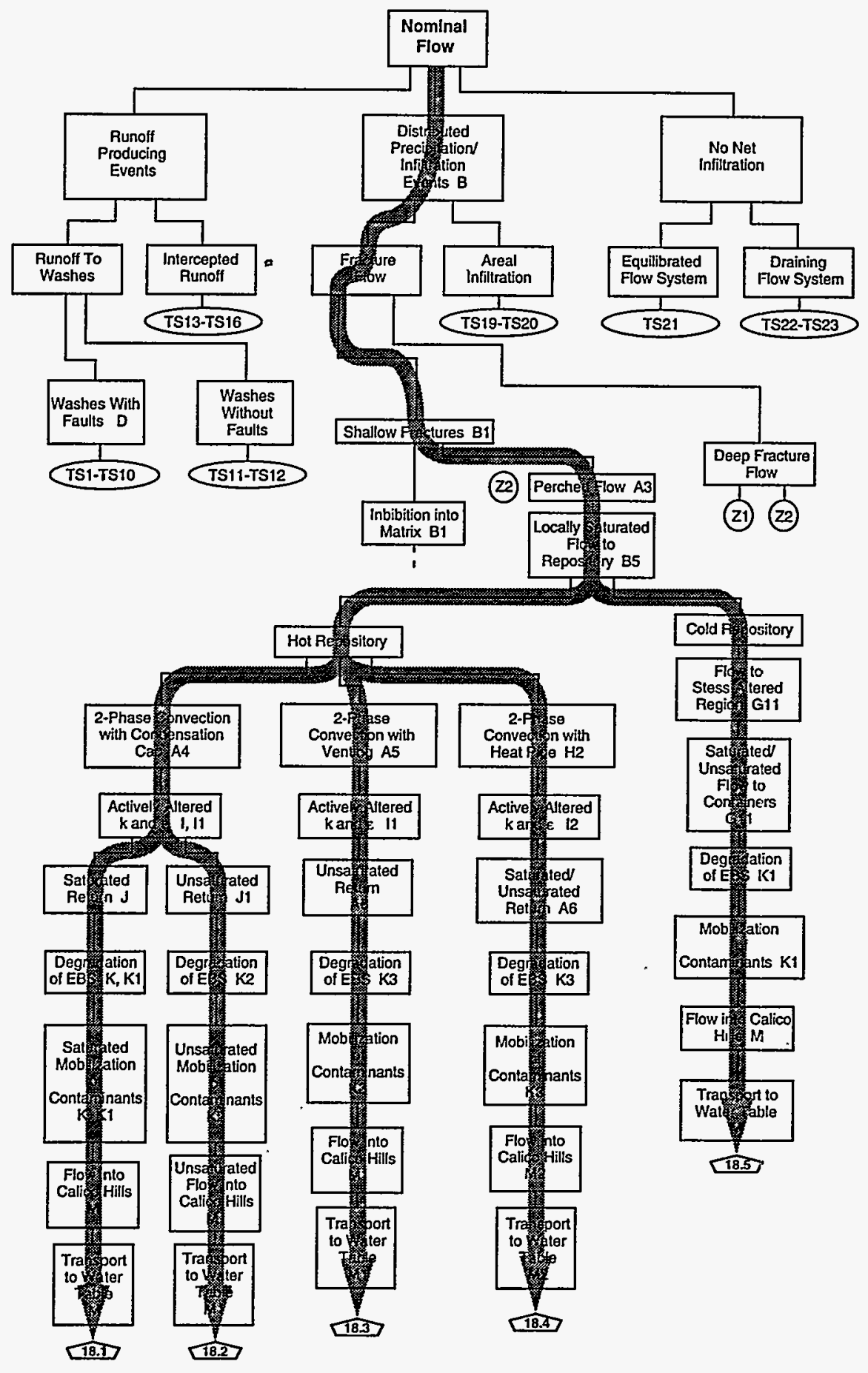

b. Scenario pathways, showing the relationship of the tree segment to the overall event tree for nominal flow. 
unsaturated/locally saturated flow sketched in Sketch M1. The dryout zone should influence movement from the saturated flow system (feeding fractures) to the unsaturated flow system. Path 18.3 describes a scenario consisting of Sketches B, B1, A3, B5, A5, I1, A6, K3, and M1.

The fourth path under a hot repository, Path $\widehat{18.4}$, considers establishment of a heat-pipe regime around the waste containers and possibly around individual drifts (Sketch H2, p. 49), as discussed above. The active region in which the heat-pipe regime functions will have its hydraulic properties altered continually (Sketch I1). When the containers have cooled sufficiently to extinguish the heat pipe and the vaporization isotherm retreats inside the containers, locally saturated flow to the repository can resume (Sketch A6). Path $\widehat{18.4}$ refers to saturated/unsaturated return because, although the source is locally saturated, any dryout associated with the heat pipe may convert the flow to unsaturated. The container has been exposed to corrosive agents continually since steam has been circulating past the container through the annulus while the heat-pipe regime was functioning. In this path, return of liquids adds to degradation of the EBS and begins to mobilize contaminants (Sketch K3). Mobilization results from both locally saturated flow and unsaturated flow as suggested in Sketch K3. The path continues with flow into the Calico Hills units and on to the water table (Sketch M2). Sketch M2 is intended to include any local resaturation by dying heat pipes, the perched water flow to the waste containers and the saturated and unsaturated transport to the water table. This closes Path $\widehat{18.4}$, which consists of Sketches B, B1, A3, B5, H2, $\mathrm{I} 2, \mathrm{~A} 6, \mathrm{~K} 3$, and $\mathrm{M} 2$.

\subsubsection{Cold Repository, Path 18.5}

The last consideration for interaction of this perched-water flow with the repository is for the case of a cold

repository (Path 18.5). The flow must continue through the stress-altered region to reach the waste containers (Sketch G11, p. 128). There is always some imbibition by the matrix, so the flow has a component of unsaturated flow. The two-phase flow during the hot period has altered conductivity and storativity in the rock. The durability of these alterations in the new water depends on the thermodynamic stability of the minerals at these lower temperatures. Whatever the state of the EBS due to its history, these locally saturated-flow fluids arrive to accelerate degradation and mobilize the contaminants (Sketch $\mathrm{K} 1$ ). Contaminants are carried on into the Calico Hills units and down to the water table (Sketch M). Path 18.5 is the scenario described by Sketches B, B1, A3, B5, $\mathrm{G} 11, \mathrm{~K} 1$, and $\mathrm{M}$.

\subsubsection{Deep Fracture Flow (Reaching Topopah Spring Units)}

Tree Segments 17 and 18 indicate another possible set of paths for the case that the fractures are not shallow but descend to the depth of the Topopah Spring Units. In this case, the paths are similar to those for shallow fractures; only the starting place is altered. Therefore we refer to the previous discussion for Paths $\mathrm{Z} 1$ and $\mathrm{Z} 2$ or alternatively to the discussion for flow down fractures to the Topopah Spring units, which is given in the section on 
interception of runoff. The arguments are the same as those given earlier for interception of runoff by fractures that conveyed fluids to the Topopah Spring units.

\subsection{Areal Infiltration}

Distributed infiltration can occur alternatively by areal infiltration in soil or rock. The discussion of paths involves similar paths as in Branches $\mathrm{Z} 1$ and $\mathrm{Z} 2$. This infiltration is essentially on kind of imbibition, as given in $\mathrm{Z1}$.

Whether a perched-water flow system-a locally saturated flow-might be formed in response to areal infiltration is not known but is included for completeness. Formation of this condition appears to depend on strong contrasts between hydraulic properties for contiguous units. The above discussions will not be repeated; however, the path structure is shown in Tree Segments 19 and 20. We refer to the indicated cartoons for detail. In the case of sufficient damping (and spreading of the unsaturated-flow plume), the paths of Tree Segments 19 and 20 reduce to a steady-state flux arriving at the repository-just the de facto reference scenarios mentioned earlier.

This completes the discussion of distributed infiltration. 


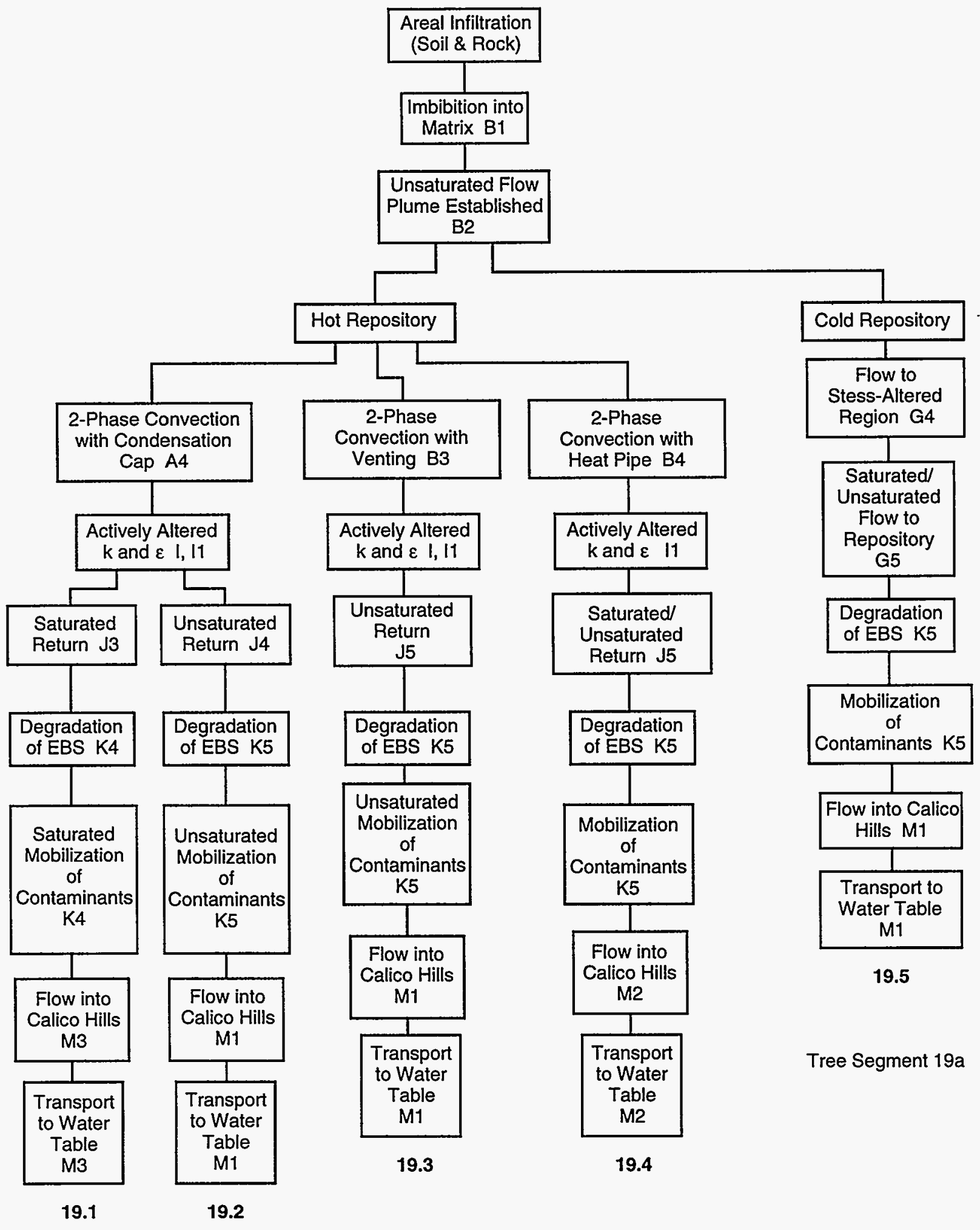

a. Events and their relationships, with cross-references to sketches illustrating each event.

Tree Segment 19. Nominal Flow, Distributed Infiltration, Areal Infiltration, Matrix Imbibition, Unsaturated Flow Plume. 


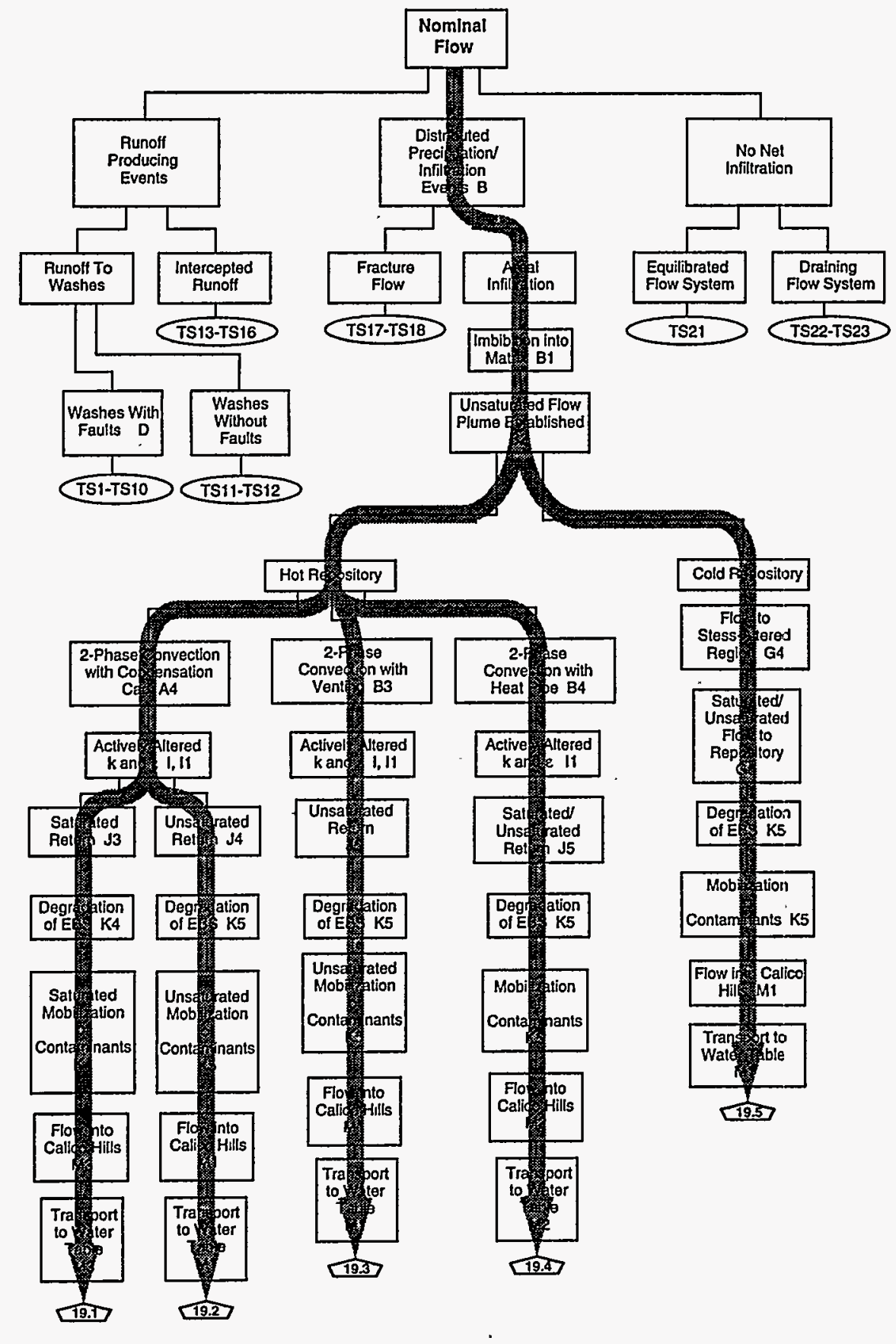

b. Scenario pathways, showing the relationship of the tree segment to the overall event tree for nominal flow. 


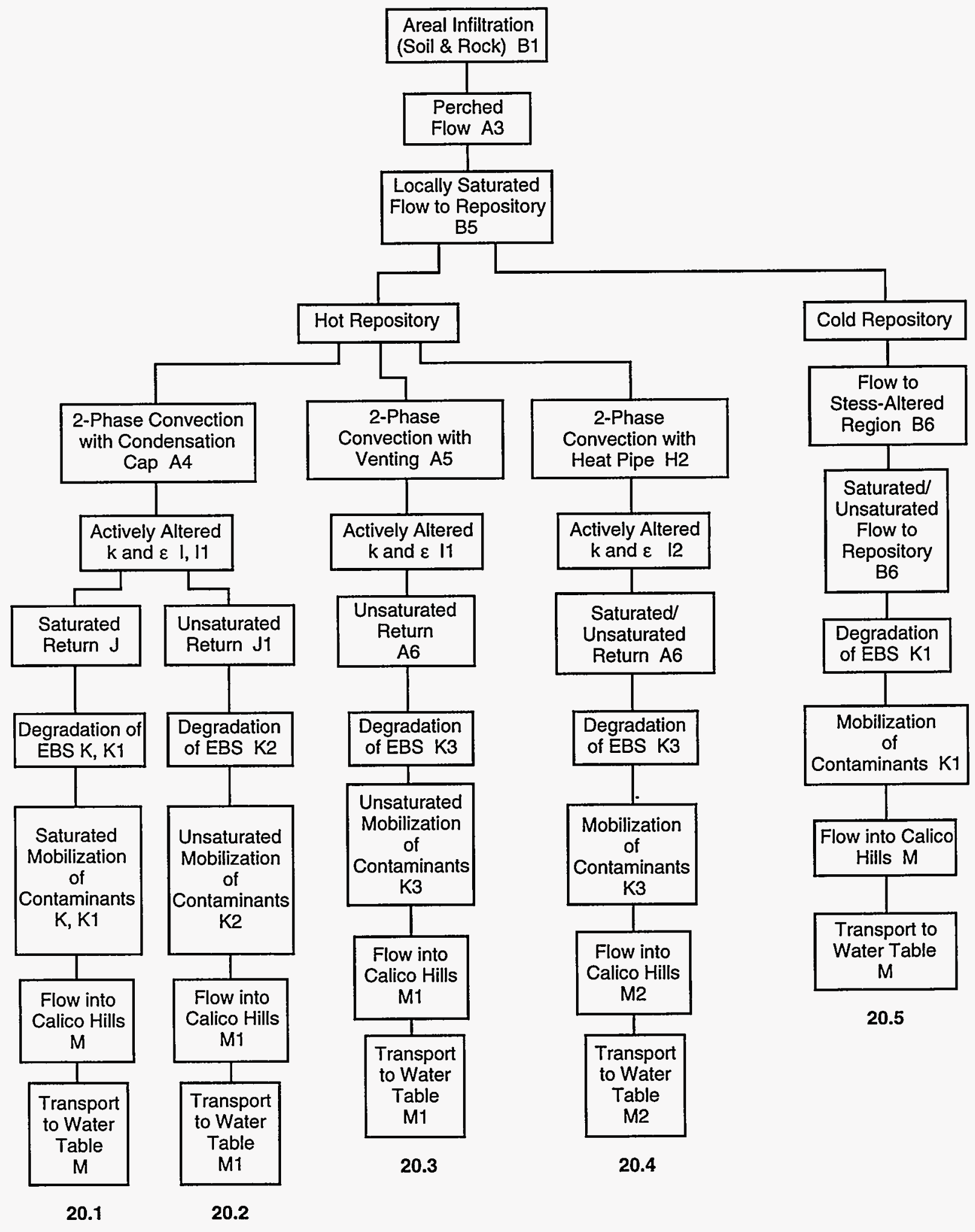

a. Events and their relationships, with cross-references to sketches illustrating each event.

Tree Segment 20. Nominal Flow, Distributed Infiltration, Areal Infiltration, Perched Flow, Locally Saturated Flow to Repository. 


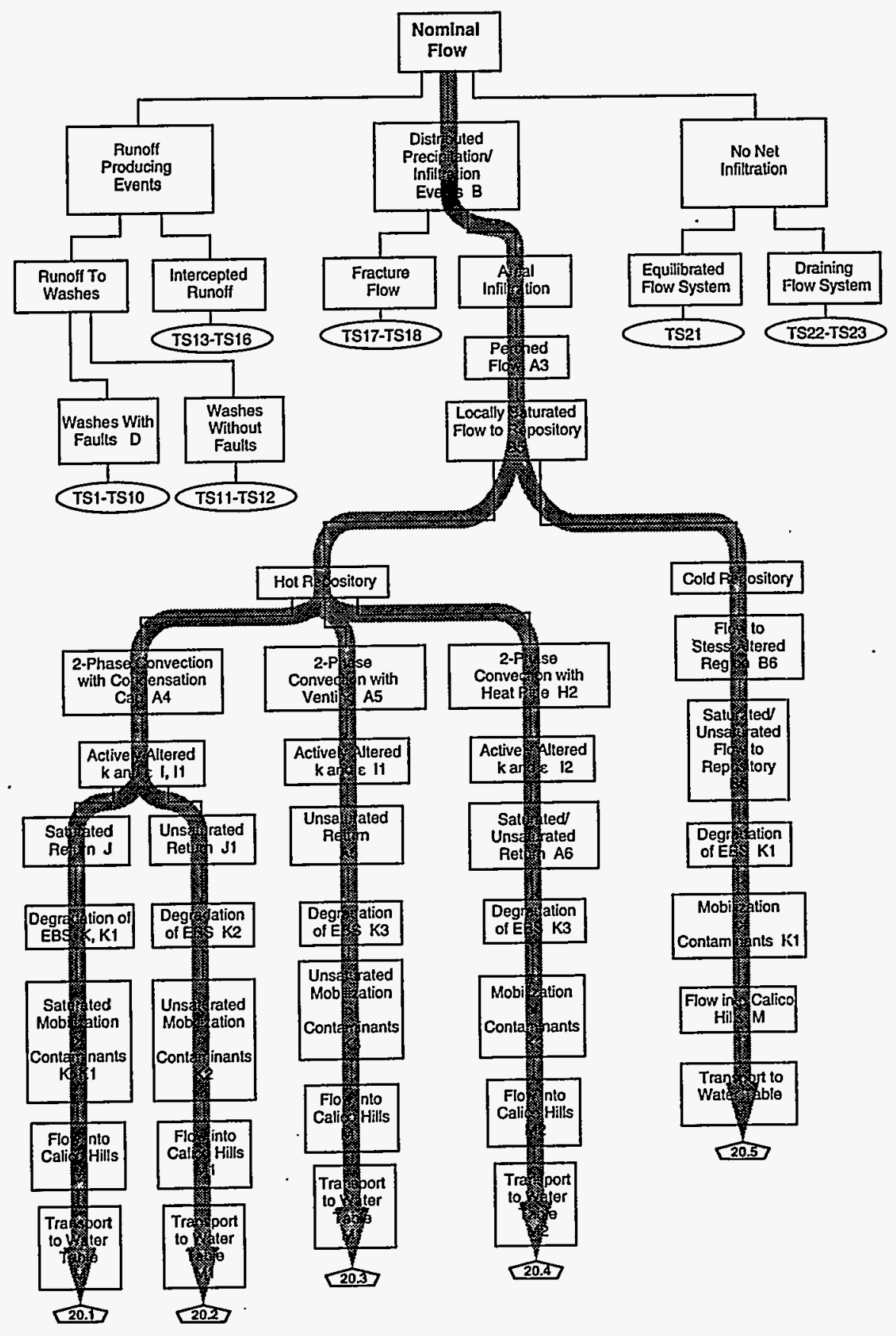

b. Scenario pathways, showing the relationship of the tree segment to the overall event tree for nominal flow. 


\section{NO NET INFILTRATION}

The third major branch below "Nominal Flow" in Figure 3 (p. 16), "No Net Infiltration," describes the circumstance that no net infiltration from the surface reaches repository depth. All infiltrating liquid either evaporates, is transpired, or is diverted in the near-surface away from the repository. A substantial amount of fluid is already resident in Yucca Mountain; in fact, some units closely approach saturation. If there is no infiltration below shallow depth, either the mountain must be in equilibrium or it must be draining. If the flow system is in equilibrium, it must be balancing the addition of any new water by vapor transport from the water table and vapor influx with the removal of water by gas flow through the mountain (Weeks, 1988; Thorstenson et al., 1989).

If the mountain is draining, and some numerical simulations suggest this (Gauthier, 1993), then various strata are not in equilibrium and have saturations different from field capacity. Therefore the repository would be inserted into an already-diminishing flow field.

\subsection{Equilibrated Flow System}

The "Equilibrated Flow System" will be discussed first. An equilibrated flow system lacks net liquid movement; rather fluid is redistributed by vapor movement. This may imply a well-connected fracture permeability, so that water table fluctuations and barometric pumping are adjusted for. High fracture permeability could allow any movement of water vapor from the mountain to be compensated by evaporation from the water table. It is into this equilibrated flow system that a hot repository will be inserted. Tree Segment 21 (p. 158) shows four paths developed from the three modes of interaction of the repository with the flow system. The first two paths, 21.1 and 21.2, are developed around formation of a condensation cap above the repository. Such a condensation cap (Sketch A4, p. 117) is a consequence of two-phase flow driven by the thermal load of the waste. However, in this case, formation of such a cap is less likely than all the earlier discussions (above) of a hot repository. High fracture permeability would probably allow venting of vapor, although some values of heat flow, water content, and permeability might inhibit venting. This circumstance could be further examined using experiments and numerical simulations. Continuing on Paths $\widehat{21.1}$ and $\widehat{21.2}$, we find the element "Actively Altered k, $\varepsilon$ " (Sketches I, I1, pp. 15 and 36), which describes changes to permeability and porosity caused by precipitates produced in the two-phase flow: Paths 21.1 and 21.2 divide, describing different modes of return flow (reflux) from the condensation cap. Path $\widehat{21.1}$ follows the saturated-flow return, where the reflux occurs as locally saturated flow or by saturated flow by the composite model (Sketch J, p. 37). Such return flow is possible only after waste containers have cooled. This reflux reinvigorates "Degradation of the EBS" (Sketch K, p. 38), which began at emplacement. Saturated mobilization (Sketch $\mathrm{K}$ ) follows and the contaminants are carried through the Topopah Spring units into the Calico Hills units and then down to the water table (Sketch M, p. 40).

Path 21.2, follows the unsaturated return (Sketch J4, p. 66) from the condensation cap to the waste containers. Further "Degradation of the EBS" (Sketch K2, p. 43) depends on physical contact between container and 
rock-some spall is indicated in the sketch to accomplish this contact. Contaminants are mobilized in the unsaturated flow field (Sketch K2) and transported through the Topopah Spring units to the Calico Hills units and through the Calico Hills unit to the water table (Sketch M1, p. 44).

The next path, 21.3, presumes that the consequence of the thermal load is venting of water vapor to the atmosphere. Measurements of atmospheric flow in the upper mountain (Weeks, 1988; Thorstenson et al., 1989) suggest a pervasive high permeability. In the case of an equilibrated flow system, venting might be a preferred behavior (Sketch B3, p. 144). Path 21.3, continues with the "Actively Altered k, $\varepsilon$ " (Sketch I1) that is expected to occur in all two-phase flow. The return of fluid to waste containers, which can only occur when the containers have cooled, is both by the liquid pathways (saturated and unsaturated, Sketch J2, p. 47) that exist and by the vapor transport this branch is intended to discuss. Once fluids have returned, "Degradation of the EBS" (Sketch K2) can proceed and there can be mobilization of the contaminants in this new flow system. Recall that in this scenario the old, prerepository liquid flow system was essentially static. The thermal gradient even for a cold repository will be substantially different from the original geothermal gradient over the 10,000 years of regulatory interest. If this new gradient coupled with mechanical changes around drifts is not able to generate liquid flow to the water table, there is no transport. Presuming that there is movement of liquid, we continue Path 21.3, with "Saturated/Unsaturated Mobilization of Contaminants" (Sketch K2), "Flow into Calico Hills" (Sketch M1), and "Transport to the Water Table" (Sketch M1).

The fourth path, 21.4, is concerned with establishment of a heat pipe, a two-phase flow system with recirculation (Sketch B4). Alteration of conductivity and storativity by precipitation and dissolution (Sketch I2, p. 50) are possible consequences of the two-phase flow. Presumably there will be dryout around the waste containers. It is unclear whether a dryout zone will persist. In any case, the return of liquid is described as unsaturated/ saturated return, in order to account for heat-pipe and vapor-driven reflux (Sketch H2). "Degradation of the EBS" (Sketch K2) occurred during the functioning of the heat pipe and is invigorated by the return of fluids to the rock. The element "Saturated/Unsaturated Mobilization of Contaminants" (Sketch K2), could include local saturation of the rock around a container by the functioning of a heat pipe with a decaying source. Because the total water volume per container is then limited, one would expect contaminant fingering or locally saturated transport until the water volume is exhausted. Flow of such fingers and locally saturated transport to the Calico Hills units and the water table are suggested in Sketch M2.

Other branches below "Nominal Flow" include paths describing release from a cold repository as a result of events or processes bringing or redirecting fluids to the waste containers. This branch has no infiltration event-the only event is cooling of the repository. This cooling was necessary for liquid return in Paths 21.1 through 21.4, and it appears thus that any cold repository considerations are already included. The scenarios in Tree Segment 21 are as follows: 


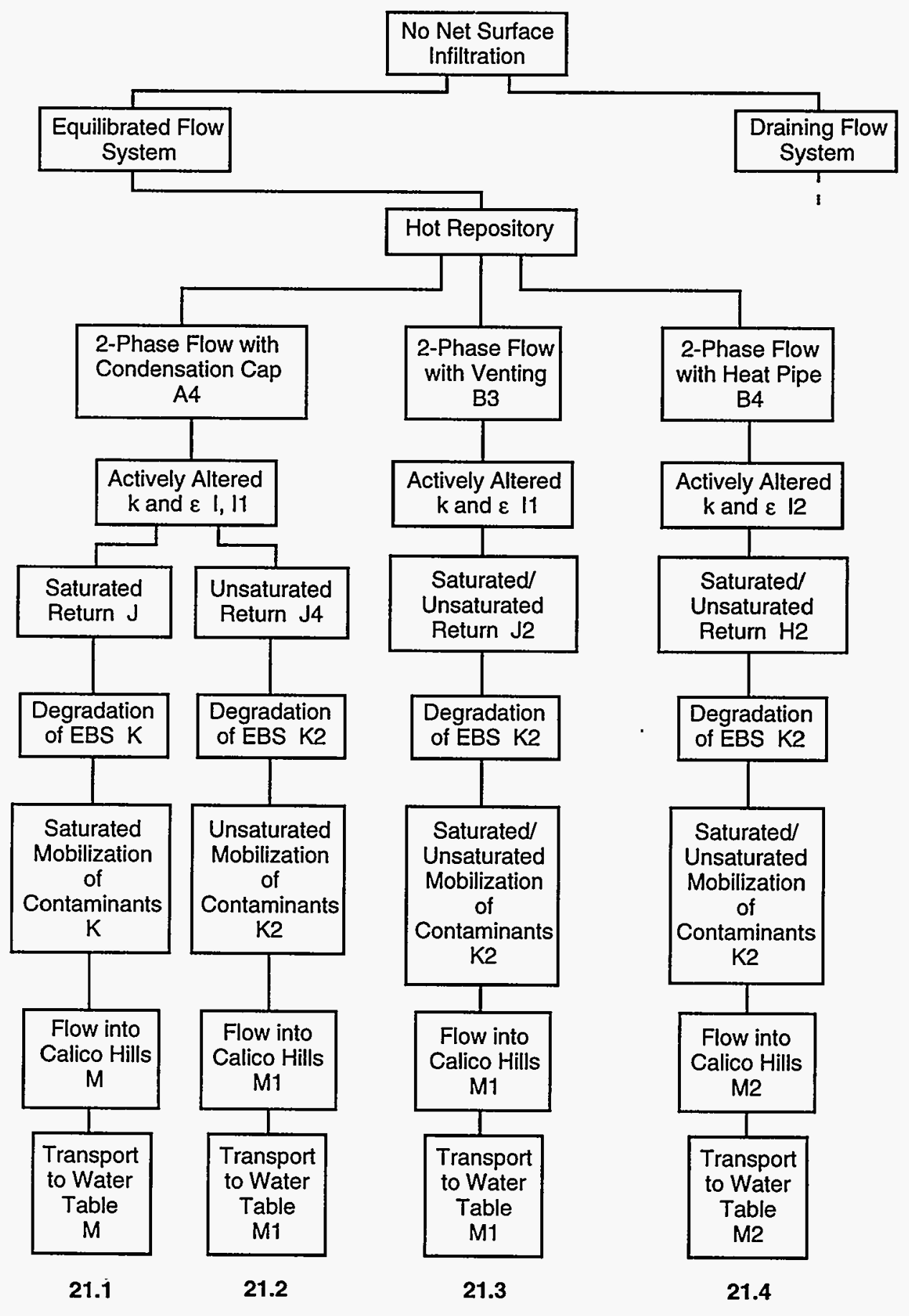

a. Events and their relationships, with cross-references to sketches illustrating each event.

Tree Segment 21. Nominal Flow, No Net Infiltration, Equilibrated Flow System. 


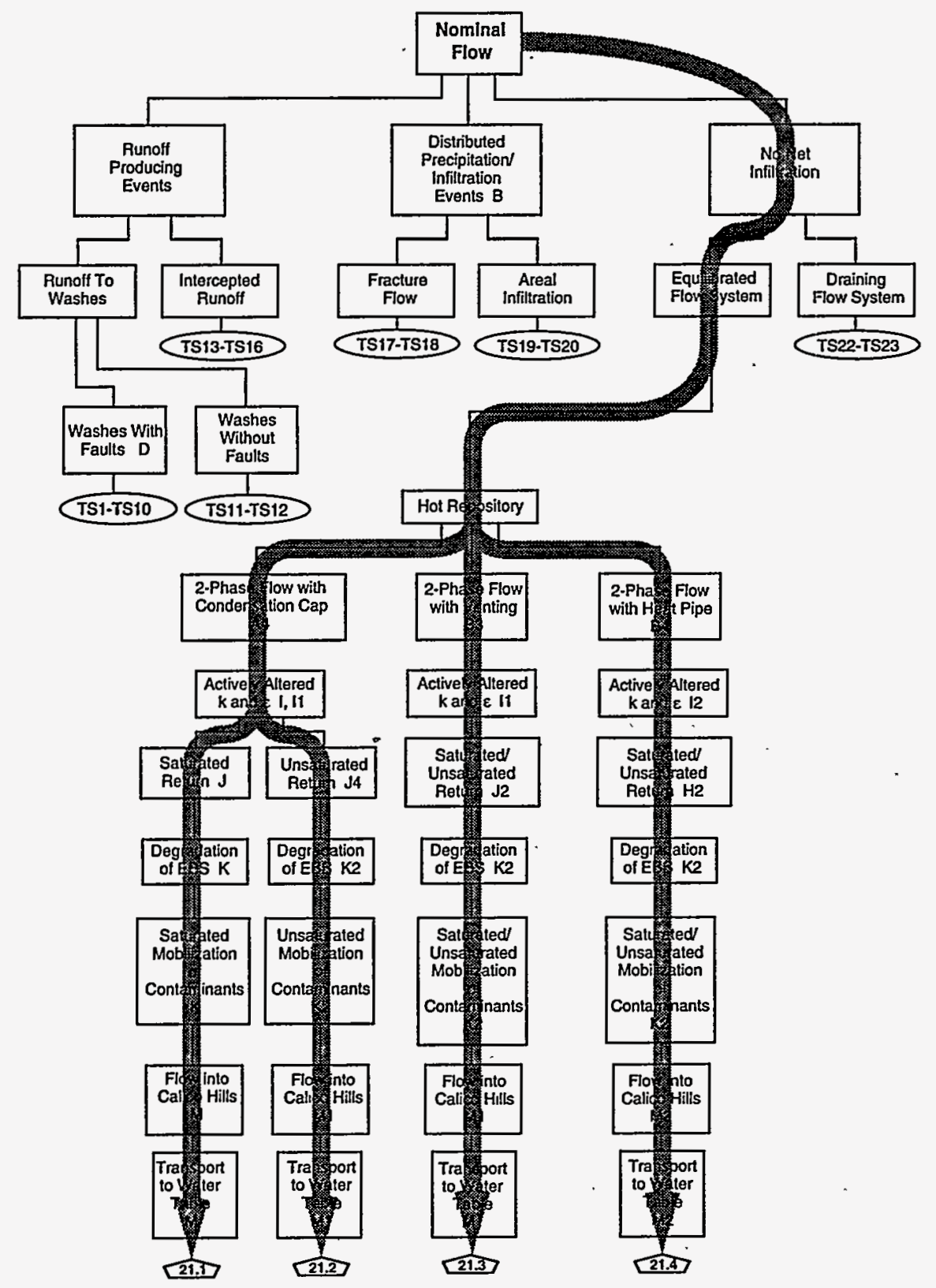

b. Scenario pathways, showing the relationship of the tree segment to the overall event tree for nominal flow. 
A4, I, I1, J, K, M

A4, I, I1, J4, K2, M1

B3, I1, J2, K2, M1

B4, I2, H2, K2, M2
Path 21.1

Path 21.2

Path 21.3

Path 21.4

\subsection{Draining Flow System}

The second branch below "No Net Surface Infiltration" is for the case that the liquid already in the mountain is draining to the water table. Since some hydrologic units in the vadose zone are apparently above field capacity at this time, recharge occurred sometime in the past. A draining flow system, whether locally saturated (Paths 22.1] through 22.6) or unsaturated (Paths 23.1 through 23.5), provides sources of liquid to interact with the repository. In earlier discussions above, the initial concern on every path was the mode of infiltration, because that mode constrained the volume of liquid and rate of arrival for any event producing infiltration. The mode of infiltration described and limited the source of new liquid available to interact with the repository. In this branch, the source is limited by the amount of liquid in the mountain at the time of waste emplacement. Other considerations, whether flow is unsaturated or locally saturated, are the same. The discussions of Paths 22.1 through 23.5 are essentially the same as paths considered earlier and we will not repeat them. The scenarios in Tree Segments 22 (p. 162) and 23 are as follows:

A4, I, J, K, K1, M

A4, I, J1, K5, M1

$\mathrm{A} 5, \mathrm{I1}, \mathrm{A6}, \mathrm{K} 2, \mathrm{~K} 3, \mathrm{M} 1$

$\mathrm{H} 2, \mathrm{I} 2, \mathrm{~A} 6, \mathrm{~K} 3, \mathrm{M} 2$

G11, N, G5, K6, M1

G11, I3, K1, M

$\mathrm{A} 4, \mathrm{I}, \mathrm{I} 1, \mathrm{~J} 3, \mathrm{~K} 4, \mathrm{M} 3$

A4, I, I1, J4, K5, M1

B3, I, I1, J5, K5, M1

B4, I1, J5, K5, M2

G5, K5, M1
Path 22.1

Path 22.2

Path 22.3

Path 22.4

Path 22.5

Path 22.6

Path 23.1

Path 23.2

Path 23.3

Path 23.4

Path 23.5 


\section{Saturated Zone}

The saturated zone provides a means of transport to the accessible environment for contaminants that reach the water table. Three issues are of compelling interest concerning ground-water flow within the saturated zone: how the saturated zone at Yucca Mountain is coupled to the unsaturated zone; the correct representation of the saturated zone; and how the coupling between the unsaturated and saturated zones will be affected by the repository.

The current coupling of the unsaturated and saturated zones is unknown; the flux is so low and partial saturation of some units so high that the mountain is probably in a transient state, possibly since the last pluvial cycle. Low flux makes measurement difficult. The working presumption in performance-assessment studies by the Yucca Mountain Project is that the unsaturated zone is currently draining to the saturated zone in some areally distributed fashion. It is possible, however, that the unsaturated zone flow system is constrained by vapor movement under the geothermal gradient, with no net downward flux (Montazer and Wilson, 1984, 1985). Numerical experiments have examined downward and lateral flux both as a function of increased flux at the repository horizon and for increased infiltration (Barnard and Dockery, 1990; Barnard et al., 1992; Eslinger et al., 1993; Wilson et al., 1994). These calculations depend on modeling interpretations and biases and on arguable values for parameters. At present there seem to be no data showing elevated heads at the water table; such data, if they existed, could be interpreted as evidence of flux in the unsaturated zone. The value appropriate for unsaturated-zone flux is being addressed by site characterization; in the meantime best estimates are bounds established by examination of physical parameters of various units. A definitive statement about coupling between the unsaturated and saturated zones is not available.

Studies of the saturated zone show an apparent high gradient just north of the potential site and a remarkably flat gradient through and southeast of the site (Czarnecki, 1989; Fridrich et al., 1991; Sinton, 1989). This flow system is interpreted to drain to the south-southwest to Franklin Lake playa (Czarnecki, 1990). Existing data-from four wells constraining the high gradient, a number of distributed wells, and geologic mapping and studies-support a number of different interpretations of the flow system in the saturated zone (Sinton, 1989; Czarnecki, 1989; Fridrich et al., 1991). Sinton (1989) suggested five possible local structural controls that offer barriers to flow: (1) faults, (2) a dike, (3) a facies change, (4) fracture orientation, and (5) a combination of these phenomena. Czarnecki (1989) added several ideas, including stratigraphic thickening of transmissive units downgradient and a semiperched water zone.

Fridrich and coworkers (1991) suggested a different structural constraint, namely, a drain in the form of a buried graben that redirects most of the fluid in the saturated tuffs down to the underlying carbonate aquifer (Fridrich et al., 1991). In this scheme, water flows beneath the repository in the carbonate units, upwelling along faults to return some water to the saturated tuffs. This interpretation effectively removes the repository from the saturated-zone flow system. It appears to be consistent with published isotopic data (Benson et al., 1983), watertemperature data, and aeromagnetic data (Fridrich et al., 1991). 


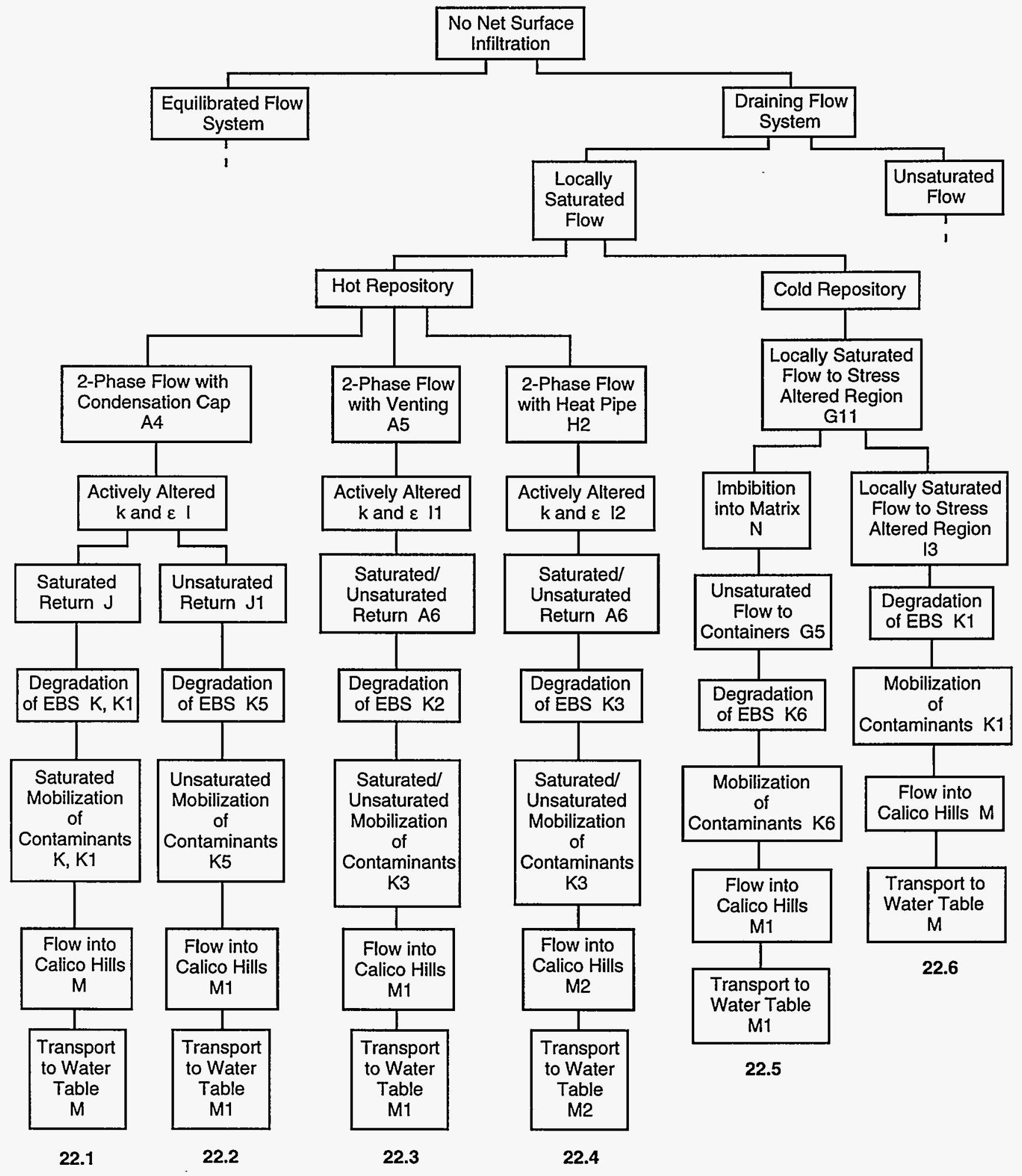

a. Events and their relationships, with cross-references to sketches illustrating each event.

Tree Segment 22. Nominal Flow, No Net Infiltration, Draining Flow System, Locally Saturated Flow. 


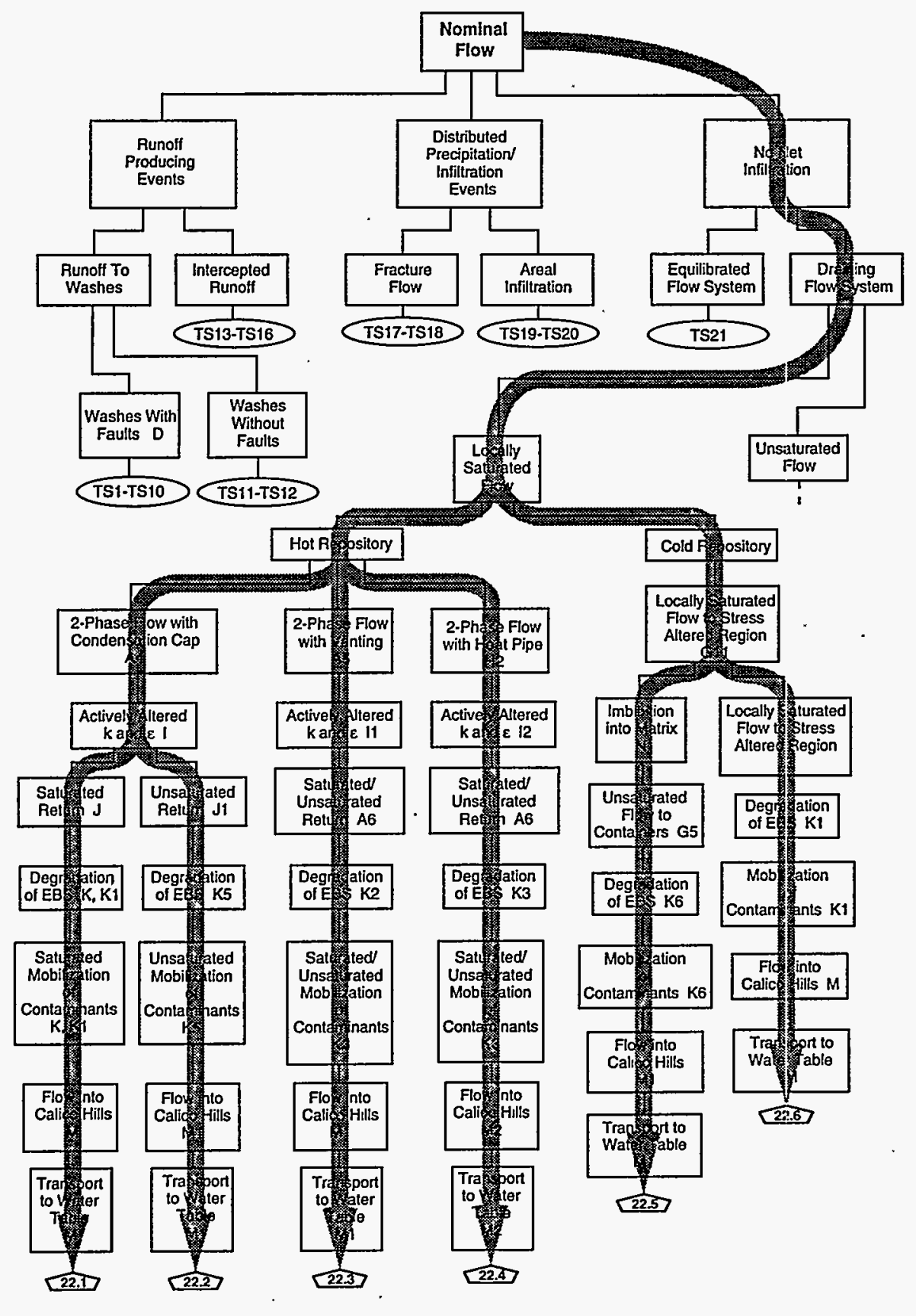

b. Scenario pathways, showing the relationship of the tree segment to the overall event tree for nominal flow. 


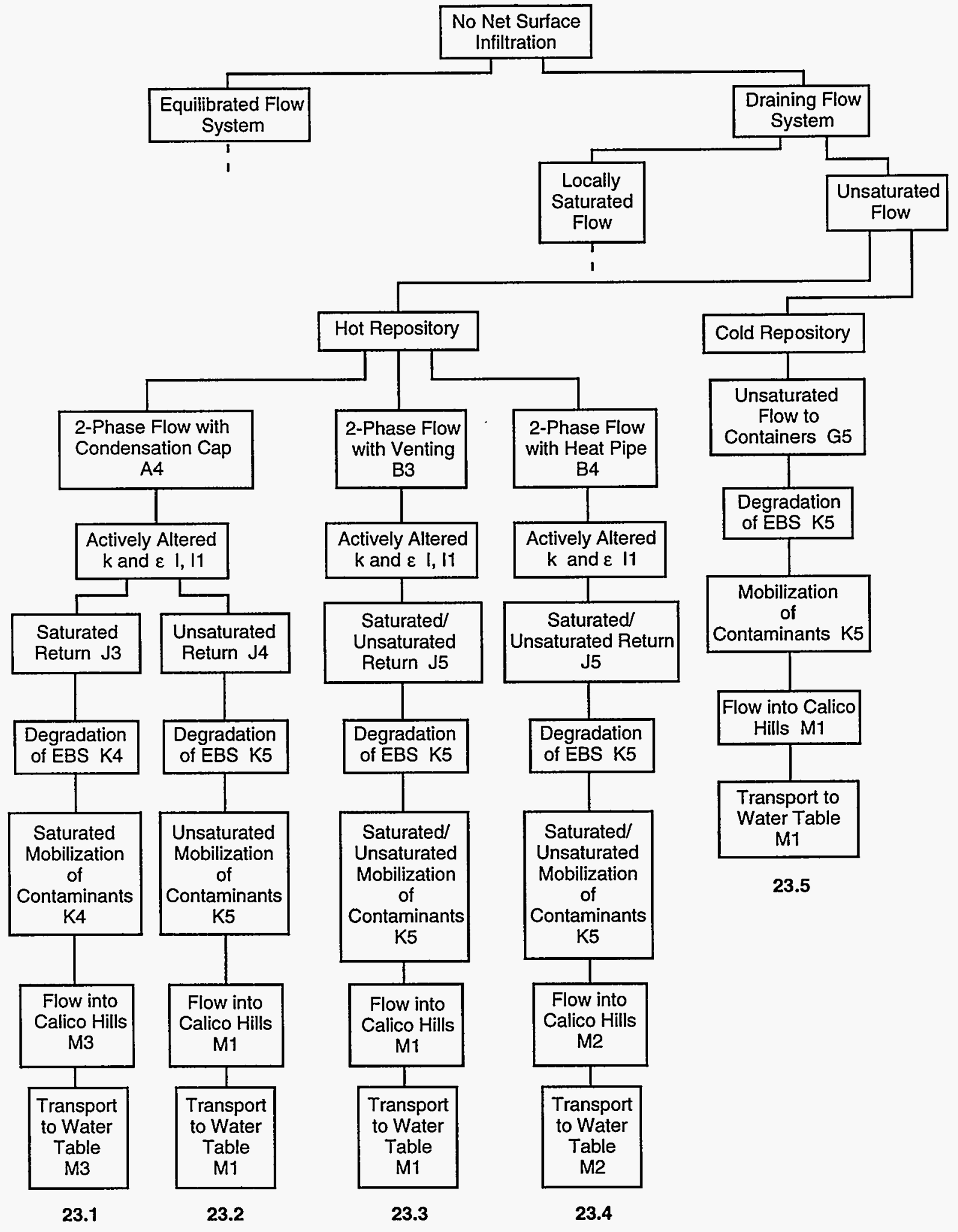

a. Events and their relationships, with cross-references to sketches illustrating each event.

Tree Segment 23. Nominal Flow, No Net Infiltration, Draining Flow System, Unsaturated Flow. 


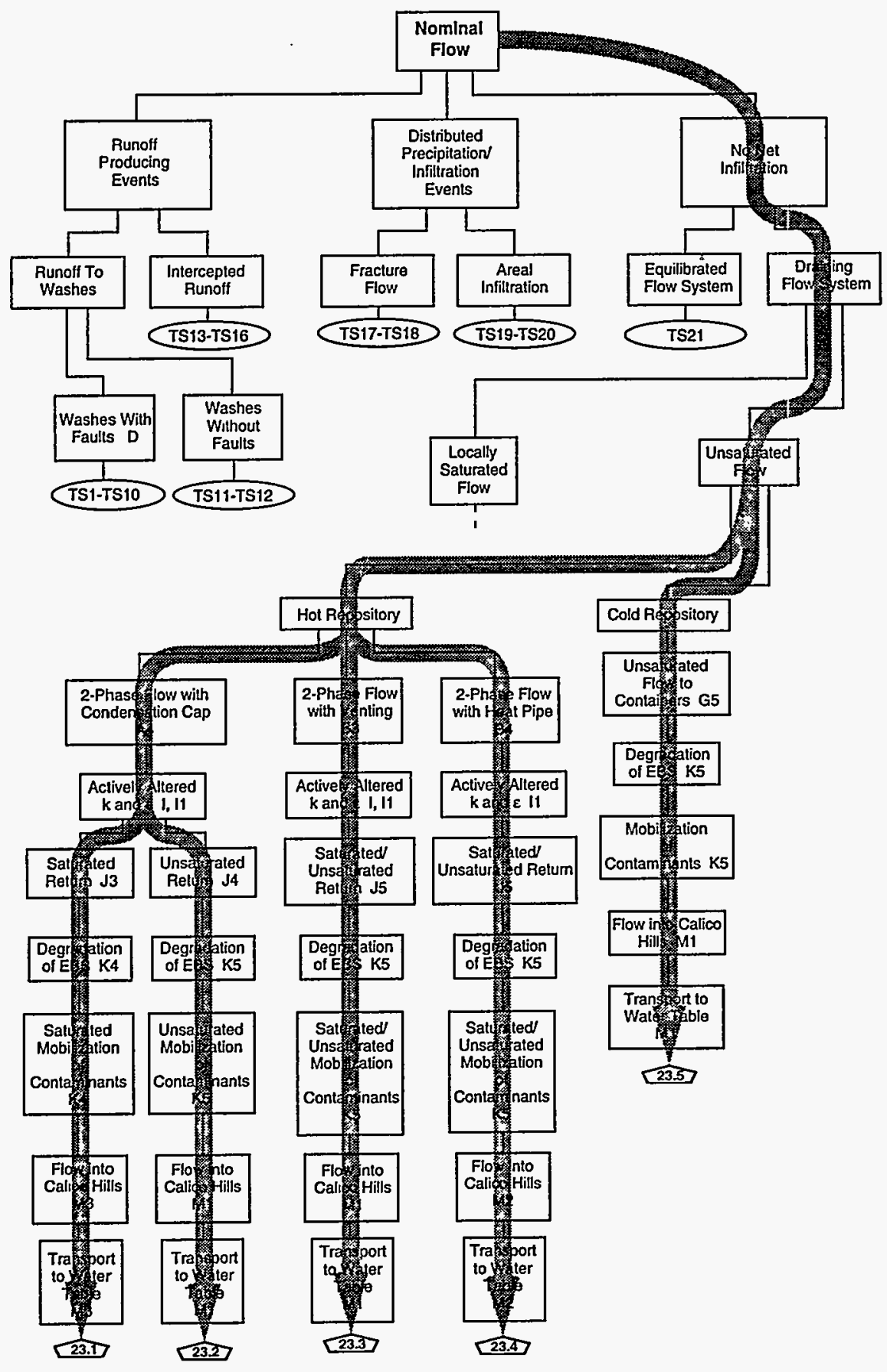

b. Scenario pathways, showing the relationship of the tree segment to the overall event tree for nominal flow. 
The cause of the gradient is of concern for two reasons. First, the controlling structure may not be durable over the repository lifetime, in which case modeling of contaminant releases that depends in some way on the continuing existence of the gradient would be flawed. Second, one interpretation effectively decouples the saturated tuffs from the regional saturated flow system (Fridrich et al., 1991), implying that water in the saturated tuffs is stagnant. Such decoupling raises the possibility that releases of radioactive waste from the repository to the water table would not lead to release to the accessible environment, in contrast to the usual assumption.

For releases from a repository that reach the water table by some means, the questions of where contaminants are going and when they might be detected at the accessible environment remain open. Early estimates (Sinnock et al., 1987) gave short times for straight-line travel down a defined flow tube. These estimates were intended for comparison of residence times in the saturated zone with those in the unsaturated zone based on data and interpretations then available. Although a well-test analysis provided the hydraulic conductivity, the estimate was flawed because the porosity used to obtain velocity was the estimated fracture porosity rather than an effective porosity consistent with the well test. No estimate of an effective porosity was or is available; however, it would be expected to be substantially larger than the fracture porosity, which would increase the time for straight-line travel down the flow tube accordingly. More recent three-dimensional modeling (Barr and Shannon, 1994; Wilson et al., 1994, Ch. 11) demonstrates that at least two different calibrated models of the saturated-flow system are consistent with currently available data on the site. The two models, which are based on different conceptual models of the saturated flow system, give rise to differing breakthrough curves for a non-retarded contaminant. In each case the calculated travel times typically are substantially longer than those of Sinnock's earlier model. The ability to construct multiple self-consistent models suggests that data on the saturated zone may be inadequate for licensing at this time, if indeed the saturated zone is important to releases at all. Moreover, the three-dimensional modeling indicates that contaminant concentrations vary substantially in time and space along the boundary of the accessible environment: contaminant breakthrough time alone cannot be used to show that the release standards have been violated.

Other, more subtle questions about the saturated zone remain, for example the mixing depth. Contaminants arriving at the water-table mix with water in the saturated zone, forming a contaminant plume extending out from the point of arrival. This plume mixes horizontally and vertically with the flow system. U.S. Geological Survey well tests have examined transmissivity of zones below the water table. Some zones accept fluid readily, others essentially not at all. This represents inhomogeneity in transmissivity and alters the flow trajectory of contaminants and thus their exposure to the rock matrix and to the fracture network. The effective mean residence time of contaminants within the protected zone is likely to be much larger than any straight line flow-tube transit time.

The third issue is how the coupling between the unsaturated and saturated zones is affected by the presence of the repository. The repository alters both the stress state and the thermal state of the surrounding rock. The repository openings alter the stress state around those openings and stress alteration is relieved by formation of fractures (Sketch G, p. 10). The waste provides sufficient heat to elevate the temperature past vaporization of water at some distance from the waste containers. The occurrence of two-phase flow, establishing a heat-pipe regime 
(Sketch H2, p. 49) or a condensation cap (Sketch J, p. 37), moves substantial volumes of fluid around the repository. Interaction between the influx from recharge and the fluid moved by two-phase flow depends on the relative volumes and flow rates and on the location of the influx (e.g., Sketches G2 and G6, pp. 63 and 123). Since thermal output of the waste is time dependent, the interaction is also time dependent. The water table is a few hundred meters below the repository. For a large-enough thermal load, the water table might participate in the two-phase flow, acting as a source of fluid well beyond that available in the unsaturated zone rocks. Further, the interaction of the repository with the saturated zone may include development of convective flow in the saturated zone and temperature-dependent chemical alterations of the hydraulic properties of the rock. Participation of the water-table aquifer and the importance of changes will be matters for calculation when more detailed, site-specific data become available during site characterization. The two-phase flow occurring around the repository alters the porosity of the matrix and the fractures. Evaporation leaves precipitates to fill openings. Precipitates and new fractures are expected to substantially alter details of how the medium transfers fluids from the current state. In short, the transfer function that the repository forms between the influx and the water table is itself a transient function that is likely to differ somewhat from the pre-emplacement state. How it differs is a matter of current study and future experimentation.

In summary, the connection between unsaturated zone and saturated zone is based on bounding estimates built from sparse data, hydrogeologic inference from the region, and general principles. 


\section{OPEN ISSUES}

A number of issues concerning the movement of fluids through Yucca Mountain have been left open in the text. These issues are best resolved by laboratory or field tests. Because we cannot be certain that data on any specific scenario will be needed to demonstrate satisfactory repository performance, it is difficult to predict which issues will have to be resolved experimentally, however. Each scenario must be considered as a whole. If one part of a scenario can be shown to be impossible or not to move contaminants toward the accessible environment, then the entire scenario disappears, even if another part of the scenario is unresolved. Below we suggest the kinds of data and modeling that are needed to resolve each issue in order to identify scenarios important to assess the performance of the repository.

In this chapter we enumerate the issues that have arisen in the course of developing this document. They are labeled with the couplings thought to be important. Three sets of issues are identified by couplings and two sets by general categories of flow type; one issue is universal:

1) Thermal-mechanical-hydrologic coupling,

2) Thermal-hydrologic coupling,

3) Thermal-mechanical-chemical-hydrologic coupling,

4) Vadose flow,

5) Saturated flow,

6) Scaling.

\subsection{Thermal-Mechanical-Hydrologic Coupling}

Thermal-mechanical-hydrologic coupling issues concern alterations in pathways and in permeability for fluids entering and leaving the mountain. Two very different scales are involved, large scale and local scale.

\subsubsection{Large Scale}

The large scale is the scale of the entire mountain. The thermal load of the repository causes thermal expansion of the rock over the entire mountain, even in areas seeing slight temperature change, as a result of mechanical coupling. Thermal expansion produces zones of compression close to the repository and zones of tension away from the repository. The exact configuration of these zones depends on repository layout, power density, overburden, rock properties, and so on. The net result is time-dependent opening and closure of fractures throughout the mountain. Ideas about possible alterations in infiltration and water movement to and away from the repository remain to be developed. There is also the possibility, also unexplored, that the thermal load could induce movement on slip surfaces in the faults at and around a repository, altering the flow paths. 


\subsubsection{Local Scale}

The local scale is the scale of the region immediately around the drifts. Classical rock mechanics (Jaeger and Cook, 1979) suggests the zone of stress alteration around a drift will extend perhaps three drift diameters into the rock. This stress alteration is eventually transformed into strain in the form of concentric and radial fractures superimposed on existing fractures. Heat adds to this stress, initially producing regions of compression around the drifts. The net result is alteration of pathways and permeability on the local scale. The importance of these local changes to redirection of fluids toward or away from waste containers remains to be examined both experimentally and computationally.

\subsection{Thermal-Hydrologic Coupling}

Thermal-hydrologic coupling issues involve the understanding of how a condensation cap develops and evolves around hot waste containers.

\subsubsection{Condensate Zone}

Formation of a condensate zone is known to depend on the relations among power density, rock properties, local saturation, permeabilities, and so on. In addition, it depends on the repository geometry, that is, shape, extent, and spacing of emplacement panels. Calculations may have already shown the essential elements of formation of condensate, although detailed data and modeling are lacking about whether there is a critical size to panels, how shedding can occur from one panel to an adjacent panel, and how shedding occurs between panels.

\subsubsection{Details Of Flow}

A zone of condensation raises unresolved questions about whether the fractures become and remain saturated or whether the matrix saturates and the fractures remain dry because of gas flow through the mountain. Laboratoryscale and larger experiments appear to be necessary to understand these details and their controls.

\subsection{Thermal-Mechanical-Chemical-Hydrologic Coupling}

Thermal-mechanical-chemical-hydrologic coupling concerns focus on changes to rock close to the waste containers (with one exception to be discussed below). 


\subsubsection{Heat Pipes}

Heat from the waste causes buoyant multiphase flow in the fluids in the rock surrounding the containers. This flow may generate heat-pipe flow around a container and later around a panel of containers. Calculations of this phenomenon are well developed for an equivalent porous medium for circumstances of no alteration of rock permeability and no chemical alterations to rock and rock fluids. The more-general cases of alterations and flow in fracture networks are lacking, because there are no data and no modeling. In addition we can only speculate on when and how fluids return to the rock, that is, whether the heat pipe induces fluid from surrounding rock to return to the vicinity of the waste.

\subsubsection{Condensate Cap}

Formation of a condensate cap with attendant dryout around the waste and possibly around the repository implies that corresponding chemical changes to the permeability of the local rock will occur. These changes have not been calculated for the condensate cap but are expected to be important for flow away from the containers and for flow back to cool containers. Alteration of the local permeability should influence how water and steam reach waste containers and therefore become important for container lifetime and corrosion.

\subsubsection{Contaminant Mobilization}

Thermal-mechanical-chemical-hydrologic coupling is expected to affect mobilization of contaminants from a corroded container. We recognize two parts to this mobilization: (1) alteration of flow (i.e., permeability changes) and reaction with the rocks, and (2) partitioning of contaminants between solutes and colloids. Current information from laboratory and field experiments and modeling are inadequate to support any firm conclusions about mobilization.

\subsubsection{Alternative Emplacement Modes}

Container design and emplacement modes different from those in the Site Characterization Plan Conceptual Design Report (MacDougall et al., 1987) are currently being considered (Benton, 1993; Doering, 1993). One option is a multi-purpose container laid along the drift floor. The drift may be backfilled, possibly with crushed tuff. Away from the emplacement drifts, the event trees would be little changed, with most of the differences accommodated in the modeling. However, locally there would be changes near the containers, and additional features, events, and process would have to be considered. For example, the local hydrothermal flow field and associated chemical interactions are expected to be different because the temperature, permeability, and porosity would differ. The presence or absence of backfill and the composition of the backfill might also be important. Because of these local 
differences, a firm design decision is required to include such alternatives in the scenarios being developed and to perform experiments that would allow pruning of irrelevant scenarios.

\subsection{Vadose Zone}

Unsaturated zone issues include both coupled problems and the behavior of fluids in the phreatic zone. We recognize the following issues to be important for understanding the behavior of fluids in the phreatic zone.

\subsubsection{Focusing of Unsaturated Flow}

The mechanisms for and constraints on focusing unsaturated flow to locally saturated flow are unknown.

\subsubsection{Fractures and Fracture Flow Channels}

In a large fracture, only a small part of the fracture, a channel, is actually expected to carry liquids. Modeling of possible fracture flow must therefore include more than the geometry of the fractures, it must also describe how the fractures carry fluid. Possibly the fractal dimension of the flow channels differs from the fractal dimension of the fractures.

\subsubsection{Evolution of Fracture Flow Channels}

It is unclear how the channels in a fracture that carry fluids will change, if they change, as the thermal environment changes. In particular it is unknown whether the original flow channels will be the preferred paths for re-establishment of flow after dryout.

\subsubsection{Geostatistical Realizations of Hydrologic Properties}

The rock surrounding a repository cannot be disassembled for inspection. There are thus relatively few data collected compared with the volume of rock around a repository. Geostatistics is one of a few methods for attempting to construct representations of the hydrogeologic structure from few data. Such data include permeability, porosity, and thermal diffusivity for the matrix, and corresponding properties and connectivity for fracture networks. We need more effort to collect data and to test realizations of the mountain as the ramps and drifts are driven. 


\subsubsection{Thermal Alteration of Hydrologic Properties}

In addition to the alteration of fracture properties mentioned above, the hydrologic properties of the matrix and fractures and the fluid itself can be altered. There are few data on thermal changes to hydraulic conductivity, for example.

\subsubsection{Unit Specific Changes on the Large Scale}

Current investigations of infiltration, specifically of the Tiva Canyon unit, suggest that certain units may control the entrance and exit of fluids from the mountain as a whole: More investigation seems to be needed, and such investigation will eventually have to include how unit properties and behavior are affected by heat and strain.

\subsubsection{Connection to the Saturated Zone}

The nature of the connection between the unsaturated and saturated zones is currently unclear. There seem to be no data establishing a connection of the two flow systems. It is important to performance assessment to establish whether a connection exists and, if not, how it might be established if percolation through the mountain increases.

\subsubsection{Flow In Faults}

We have no information on whether flow in faults or in fault zones (including gouge) is similar to flow in fractures. A better experimental understanding of the constraints and controls on such flow appears to be necessary.

\subsubsection{Flow Across Hydrologic Units}

Laboratory experiments show that contiguous hydrologic units often have quite different values of hydraulic parameters like permeability and connected porosity. Presumably fluid moves across and possibly along the contact boundaries. Exactly how flow occurs at boundaries needs further investigation, since it is a control on the residence time of fluids in a unit.

\subsubsection{Flow Within Fracture Networks}

Determination of the connectedness of fracture networks is difficult at best, especially in the vadose zone where measurement techniques and experience are lacking. That connectedness, and therefore the flow system, is likely to be altered by excavation effects (stress relief) and by heat (thermo-mechanical coupling and thermo-mechanicalchemical coupling). These changes are in addition to the "natural" changes independent of the repository due to 
weathering and changes in water flow. At this time it is not clear that single-fracture studies will be will be adequate to address these issues for fracture networks. Since the flow in fracture networks is likely to control the nearcontainer multiphase flow, studies of how flow in fracture networks occurs ought to proceed.

\subsection{Saturated Zone}

Saturated zone issues involve both the expected flow field and coupling. We can only roughly state some of these issues because we expect more to develop as experimental work provides more data and analyses develop more interpretations of the data.

\subsubsection{Fault Controls on the Flow System}

Semiquantitative calculations (Wilson et al., 1994) suggest that faults around the repository (e.g., the Solitario Canyon fault and the possible fault in Drill Hole Wash) may control the flow system and be responsible for the large hydraulic gradient north of the potential site. Determination of the hydraulic importance of these and other faults requires drilling and well tests in their vicinity.

\subsubsection{Coupling Between the Tuff And Carbonate Aquifers}

An alternative conceptual model of the saturated zone (Fridrich et al., 1991) postulates that the saturated tuffs and the carbonate aquifer are coupled and that this coupling produces the large hydraulic gradient north of the site. Such coupling could isolate the saturated zone below the potential repository site from the rest of the flow system and provide long residence times for contaminants that reach the water table. Resolution of this interpretation requires drilling in the region of the large hydraulic gradient and drilling to the carbonate aquifer.

\subsubsection{Thermal-Chemical Alteration}

In spite of the distance of the water table from the repository, some calculations seem to indicate that there will be a thermal gradient extending below the water table (Buscheck and Nitao, 1993). This thermal gradient would cause convective cells below the water table. The thermal gradient also causes differential solubility of rock constituents like silica and calcite. Circulation of dissolved constituents from warmer to colder regions beneath the heat source could alter rock permeability enough to affect transport of contaminants from the waste. One possibility is that permeability would be reduced enough to dramatically affect residence times for contaminant transport. We have no calculations and no laboratory experiments of this coupled process to decide whether it proceeds vigorously enough to change permeability significantly during the period that the heat source is able to generate convection below the water table. 


\subsubsection{Dispersion In Fractured, Porous Media}

We currently depend on the universality of scale arguments of Neuman (1990) for our estimates of dispersion coefficients. Confirmation or refutation of those arguments specifically for Yucca Mountain as a result of experiments is necessary for performance assessment. We do not know what those field studies should include at this time.

\subsubsection{Mixing Depth}

Contaminants entering the saturated zone flow system experience mixing and therefore dilution. In three dimensions it is likely that contaminants reaching different depths will have different paths and emerge at different locations in the accessible environment. We are not yet able to estimate with confidence over what depth such mixing occurs. The mixing depth is an important parameter for specifying details of release and dose.

\subsubsection{Hydrologic Properties of Unsaturated Units}

Certain properties of the unsaturated zone units, such as liquid permeability and anisotropy, are difficult or impossible to measure directly in the unsaturated zone. However, at some wells (like J13) these same units are below the water table and much more amenable to testing.

\subsection{Scaling}

There are three aspects of scaling: scaling of data, scaling of analyses, and scaling of time responses.

Rules to allow experimental results from the laboratory or from the experimental studies facility to be generalized to the scale of the repository need to be developed or need to be shown to be irrelevant for performance assessment.

As the size of the problem increases from the size of the container to the size of the mountain, some couplings of processes drop out or must be included. The criteria for those changes in the models remain to be developed.

Each of the fields describing coupled processes, thermal, mechanical, chemical, and hydrologic, have characteristic response or propagation times. These times can be orders of magnitude different and generally represent the times during which various field are effectively decoupled or coupled. We particularly lack characteristic times for chemical and hydrologic coupling. 


\subsection{Conclusion}

This discussion of items arising from these issues is by no means exhaustive. It simply presents a few key concerns that would facilitate modeling of scenarios and would help in pruning scenarios from the trees. Many items involve experimental work in the laboratory or field. We expect that a frequent result of this work will be that our concerns are shown to have been misplaced and should have been directed at another part of the problem. Determining the weakness in a given scenario is an important part of scenario construction. 


\section{REFERENCES}

Barnard, R. W., and H. A. Dockery, 1990. Performance Assessment Calculational Exercises: PACE-90: Overview and Summary, SAND90-2088C, Sandia National Laboratories, Albuquerque, NM, 9 pp. (NNA.911220.0064)

Barnard, R. W., M. L. Wilson, H. A. Dockery, J. H. Gauthier, P. G. Kaplan, R. R. Eaton, F. W. Bingham, and T. H. Robey, 1992. Yucca Mountain Site Characterization Project, TSPA 1991: An Initial Total-System Performance Assessment for Yucca Mountain, SAND91-2795, Sandia National Laboratories, Albuquerque, NM. (NNA.920630.0033)

Barr, G. E., and Sharon A. Shannon, 1994, "Constraining Local 3-D Models of the Saturated Zone, Yucca Mountain, Nevada," High-Level Radioactive Waste Management, Proc. Fifth Annual Internatioal Conf., Las Vegas, NV, May 22-26, 1994, vol. 4, pp. 1814-1821. (MOL.19941021.0005)

Benson, L. V., J. H. Robison, R. K. Blankennagel, and A. E. Ogard, 1983. Chemical Composition of Groundwater and the Locations of Permeable Zones in the Yucca Mountain Area, Nevada, USGS-OFR-83-854, U.S. Geological Survey, Denver, CO, 19 pp. (NNA.890511.0102)

Benton, H. A., 1993, "Operational Considerations in Drift Emplacement of Waste Packages," Proc. Fourth Internat. Conf., High Level Radioactive Waste Management, April 1993, Las Vegas, NV, p. 544-550. (NNA.940629. 0025)

Blejwas, T. E., 1989. "Stability of Underground Openings in the Yucca Mountain Repository," Trans. 10th Internat. Conf. Structural Mechanics in Reactor Technology, vol. R., Los Angeles, CA, pp. 153-158. (MOL.19941004.0154)

Buscheck, T. A., and J. J. Nitao, 1988. Preliminary Scoping Calculations of Hydrothermal Flow in Variably Saturated, Fractured, Welded Tuff During the Engineered Barrier Design Test at the Yucca Mountain Exploratory Shaft Test Site, UCID-21571, Lawrence Livermore National Laboratory, Livermore, CA, 31 pp. (NNA.890224.0009)

Buscheck, T. A., and J. J. Nitao, 1993, The Impact of Repository Heat on Thermo-Hydrological Performance at Yucca Mountain, UCRL-JC-114791, Lawrence Livermore National Laboratory, Livermore, CA. (NNA. 940121.0144)

Byers, F. M., Jr., W. J. Carr, and Paul P. Orkild, 1989, "Volcanic Centers of Southwestern Nevada: Evolution of Understanding, 1960—1988," J. Geophys. Research , v. 94, pp. 5908-5924. (NNA.900403.0407)

Czarnecki, J. B., 1989. "Preliminary Simulations Related to a Large Horizontal Hydraulic Gradient at the North End of Yucca Mountain, Nevada," EOS, Transactions of the American Geophysical Union, vol. 70, no. 15, p. 321. (NNA.891218.0142)

Czarnecki, J. B., 1990. Geohydrology and Evapotranspiration at Franklin Lake Playa, Inyo County, California, USGS-OFR-90-356, U.S. Geological Survey, Denver, CO, 96 pp. (NNA.901015.0195)

Doering, T. W., 1993, "Robust Waste Package Concept (Multibarrier)," Proc. Fourth Internat. Conf., High Level Radioactive Waste Management, April 1993, Las Vegas, NV, p. 551-557. (NNA.940629.0024)

Doughty, C., and K. Pruess, 1987. "Heat Pipe Effects in Nuclear Waste Isolation," Lawrence Berkeley Laboratory Annual Report, LBL-22090, Lawrence Berkeley Laboratory, Berkeley, CA, pp. 32-34. (MOL.19940805.0096)

Duebendorfer, E. M., and R. A. Black, 1992, "Kinematic Role of Transverse Structures in Continental Extension: An Example from the Las Vegas Valley Shear Zone, Nevada," Geology, vol. 20, p. 1107-1110. (MOL. 19940819.0010) 
Eaton, R. R., and D. C. Reda, 1982. "The Influence of Convective-Energy Transfer on Calculated Temperature Distributions in Proposed Hard-Rock Nuclear Waste Repositories," Radioactive Waste Management, vol. 2, no. 4, pp. 343-361. (MOL.19940819.0011)

Eaton, R. R., N. E. Bixler, and D. C. Reda, 1987. "Coupled Hydrothermal Flows of Liquid and Vapor in Welded Tuff: Numerical Modeling of Proposed Experiment," in C. F. Tsang, ed., Coupled Processes Associated with Nuclear Waste Repositories, Academic Press, San Diego, CA, pp. 409-420. (MOL.19940805.0097)

Eslinger, P. W., L. A. Doremus, D. W. Engel, T. B. Miley, M. T. Murphy, W. E. Nichols, M. D. White, D. W. Langford, and S. J. Ouderkirk, 1993, Preliminary Total-System Analysis of a Potential High-Level Nuclear Waste Repository at Yucca Mountain, PNL-8444, Pacific Northwest Laboratory, Richland, WA. (HQO.930219.0001)

Fridrich, C. J., D. C. Dobson, and W. W. Dudley, 1991. "A Geologic Hypothesis for the Large Hydraulic Gradient Under Yucca Mountain, Nevada," EOS, Transactions of the American Geophysical Union, vol. 72, p. 121. (NNA.910919.0013)

Frizzell, V. A., Jr., and J. Shulters, 1990. Geologic Map of the Nevada Test Site, Southern Nevada, Map I-2046, 1:100,000, U. S. Geological Survey. (NNA.901130.0020)

Gauthier, J. H., 1993. "The Most Likely Groundwater Flux Through the Unsaturated Tuff Matrix at USW H-1," Proc. Fourth Annual Int. Conf. High Level Radioactive Waste Management, 26-30 April, pp. 146-151. (NNA. 930702.0025)

Glass, R. J., 1989. Miller Scaling of Finger Properties in Sandy Soils: An Indirect Method for Estimating Finger Width and Velocity, SAND89-2376C, Sandia National Laboratories, Albuquerque, NM, 20 pp. (NNA.900501. 0096)

Glass, R. J., and V. C. Tidwell, 1991, Research Program to Develop and Validate Conceptual Models for Flow and Transport through Unsaturated, Fractured Rock, SAND90-2261, Sandia National Laboratories, Albuquerque, NM, 60 p. (NNA.910906.0001)

Gomberg, J., 1991, "Seismicity and Shear Strain in the Southern Great Basin of Nevada and California," J. Geophys. Research, vol. 96, p. 16,383-16,399. (NNA.920211.0032)

Grant, M. A., I. G. Donaldson, and P. F. Bixley, 1982, Geothermal Reservoir Engineering, Academic Press, New York, 369 p. (MOL.19941206.0031)

Hopkins, P. L., R. R. Eaton, and S. Sinnock, 1987. Nevada Nuclear Waste Storage Investigations Project: Effect of Drift Ventilation on Repository Hydrology and Resulting Solute Transport Implications, SAND86-1571, Sandia National Laboratories, Albuquerque, NM, 49 pp. (NNA.891019.0244)

Hunter, R. L., G. E. Barr, and F. W. Bingham, 1983. Scenarios for Consequence Assessments of Radioactive-Waste Repositories at Yucca Mountain, Nevada Test Site. SAND82-1277, Sandia National Laboratories, Albuquerque, NM, 117 pp. (NNA.891004.0364)

Jaeger, J. C., and N. G. W. Cook, 1979, Fundamentals of Rock Mechanics, 3rd edition, Chapman and Hall, London, 593 pp. (NNA.910326.0057)

Johnson, G. L., 1991. Thermal Performance of a Buried Nuclear Waste Storage Container Storing a Hybrid Mix of PWR and BWR Spent Fuel Rods, UCID-21414, Rev. 1, Lawrence Livermore National Laboratory, Livermore, CA. (NNA.911107.0015)

Johnstone, J. K., and P. F. Gnirk, 1982. Preliminary Technical Constraints for a Repository in Tuff, SAND822147C, Sandia National Laboratories, Albuquerque, NM, 6 pp. (SRX.830202.0113) 
Johnstone, J. K., A. R. Lappin, and R. R. Peters, 1982. "System Study and Performance Constraints for Repository Horizon Selection at the Nevada Test Site," Trans. Amer. Nuclear Soc., vol. 43, pp. 75-76. (MOL.19940819. 0006)

MacDougall, H. R., L. W. Scully, and J. R. Tillerson, compilers, 1987. Site Characterization Plan: Conceptual Design Report, SAND84-2641, Sandia National Laboratories, Albuquerque, NM, Vols. 1 - 6. (NN1.880902. $0014-0.0019$ )

Maldonado, F., 1990, "Structural Geology of the Upper Plate of the Bullfrog Hills Detachment Fault System, Southern Nevada," Geol. Soc. Amer. Bull., vol. 102, p. 992-1006. (NNA.910123.0022)

Montazer, P., and W. E. Wilson, 1984. Conceptual Hydrologic Model of Flow in the Unsaturated Zone, USGS/WRI-84-4345, U. S. Geological Survey, Lakewood, CO, 59 pp. (NNA.890327.0051)

Montazer, P., and W. E. Wilson, 1985. Hydrogeology of the Unsaturated Zone, CONF-8511172-6, U. S. Geological Survey, Denver, CO, 17 pp. (SRX.861119.0160)

Mualem, Y., and J. Bear, 1978. "Steady Phreatic Flow Over a Sloping Semipervious Layer," Water Resources Research, vol. 14, no. 3, pp. 403-408. (MOL.19940819.0008)

Neuman, S. P., 1990, "Universal Scaling of Hydraulic Conductivities and Dispersivities in Geologic Media," Water Resources Research, vol. 26, no. 8, pp. 1749-1758. (NNA.920320.0021)

Nicholl, M. J., R. J. Glass, and H. A. Nguyen, 1993, "Small-scale Behavior of Single Gravity-driven Fingers in an Initially Dry Fracture," Proceedings of the Fourth Internat. Conf., High Level Radioactive Waste Management, April 1993, Las Vegas, NV. (NNA.930702.0015)

Nitao, J. J., 1988. Numerical Modeling of the Thermal and Hydrological Environment Around a Nuclear Waste Package Using the Equivalent Continuum Approximation: Horizontal Emplacement, UCID-21444, Lawrence Livermore National Laboratory, Livermore, CA, 77 pp. (NNA.890317.0021)

Nitao, J. J., 1989. On the Movement of a Liquid Front in an Unsaturated, Fractured Porous Medium, Part II.Mathematical Theory. UCID-21743, Lawrence Livermore National Laboratory, Livermore, CA, 26 pp. (HQX.900216.0012)

Nitao, J. J., and T. A. Buscheck, 1989a. "On the Infiltration of a Liquid Front in an Unsaturated, Fractured Porous Medium," Proc. Amer. Nuclear Soc. Topical Meeting, "Nuclear Waste Isolation in the Unsaturated Zone," September 18-21, 1989, Las Vegas, NV, 40 pp. (MOL.19940805.0104)

Nitao, J. J., and T. A. Buscheck, 1989b. On the Infiltration of a Liquid Front in an Unsaturated, Fractured Porous Medium, Part I. UCID-21714, Lawrence Livermore National Laboratory, Livermore, CA, 44 pp. (HQX. 900216.0012)

Parsons, Brinckerhoff, Quade and Douglas, Inc., 1987. Preliminary Study for Cooldown of an Emplaced Drift, prepared by Mine Ventilation Services. (NNA.881219.0072)

Peters, R. R., and E. A. Klavetter, 1988. "A Continuum Model for Water Movement in an Unsaturated Fractured Rock Mass," Water Resources Research, vol. 24, no. 3, pp. 416-430. (NNA.890523.0139)

Pruess, K., 1986. "Vapor-Dominated Geothermal Reservoirs as Heat Pipes in Fractured Porous Rock," Lawrence Berkeley Laboratory Annual Report, LBL-20450, pp. 121-123. (MOL.19940819.0009)

Pruess, K., and Y. Tsang, 1991. "Thermal Effects on Fast Paths," memorandum to Rally Barnard, Sandia National Laboratories, April 25, 1991, from Lawrence Berkeley Laboratory, Berkeley, CA, 14 pp. (MOL.19941206. 0030) 
Pruess, K., J. S. Y. Wang, and Y. W. Tsang, 1988. Effective Continuum Approximation for Modeling Fluid and Heat Flow in Fractured Porous Tuff, SAND86-7000, Sandia National Laboratories, Albuquerque, NM. (NNA.891019.0272)

Pruess, K., J. S. Y. Wang, and Y. W. Tsang, 1990. "On Thermohydrological Conditions Near High-Level Nuclear Wastes Emplaced in Partially Saturated Fractured Tuff, Part 1: Simulation Studies With Explicit Consideration of Fractured Effects," Water Resources Research, vol. 26, no. 6, pp. 1235-1248. (NNA.910328.0073)

Ramspott, L. D., 1991. The Constructive Use of Heat in an Unsaturated Tuff Repository, UCRL-JC-105868, Lawrence Livermore National Laboratory, Livermore, CA, 9 pp. (NNA.930125.0080)

Ross, B., 1987. A First Survey of Disruption Scenarios for a High-Level-Waste Repository at Yucca Mountain, Nevada, SAND85-7117, Sandia National Laboratories, Albuquerque, NM, 147 pp. (HQS.880517.2850)

Ryder, Eric, 1993, "Comparison of Predicted Far-Field Temperatures for Discrete and Smeared Heat Sources," Proc. International High-Level Radioactive Waste Management Conf., Amer. Nuclear Society, Las Vegas, NV, pp. 841-846. (NNA.930702.0022)

Scott, R. B., and J. Bonk, 1984. Preliminary Geologic Map of Yucca Mountain, Nye County, Nevada, With Geologic Sections, USGS-OFR-84-494, U.S. Geological Survey, Denver, CO. (HQS.880517.1443)

Sinnock, S., 1982. Geology of the Nevada Test Site and Nearby Areas, Southern Nevada, SAND82-2207, Sandia National Laboratories, Albuquerque, NM, 55 pp. (NNA.870406.0282)

Sinnock, S., Y. T. Lin, and J. P. Brannen, 1987. "Preliminary Bounds on the Expected Postclosure Performance of the Yucca Mountain Repository Site, Southern Nevada," J. Geophys. Res., vol. 92, no. B8, pp. 7820-7842. (NNA.900702.0031)

Sinnock, S., Y. T. Lin, and M. S. Tierney, 1986. Preliminary Estimates of Groundwater Travel Time and Radionuclide Transport at the Yucca Mountain Repository Site, SAND85-2701, Sandia National Laboratories, Albuquerque, NM, 156 pp. (NNA.891129.0550)

Sinton, P. O., 1989. "Characterization of the Large Hydraulic Gradient Beneath the North End of Yucca Mountain, Nevada," EOS, Abstract H11B-03 0910H, vol. 70, p. 321. (NNA.891204.0065)

Thorstenson, D. C., J. C. Woodward, E. P. Weeks, and H. Haas, 1989. Physical and Chemical Characteristics of Topographically Affected Airflow in an Open Borehole at Yucca Mountain, Nevada, CONF-890928-25, U.S. Geological Survey, Reston, VA, 15 pp. (NNA.900723.0196)

Tsang, Y. W., and K. Pruess, 1987. "A Study of Thermally Induced Convection Near a High-Level Nuclear Waste Repository in Partially Saturated Fractured Tuff," Water Resources Research, vol. 23, no. 10, pp. 1958-1966. (NNA.890522.0273)

U.S. Department of Energy, 1988. Site Characterization Plan: Yucca Mountain Site, Nevada Research and Development Area, Nevada, U.S. Department of Energy, Office of Civilian Radioactive Waste Management, Washington, DC, Vols. 1 - 9. (HQO.881201.0002)

Wallace, K. G., Jr. and D. P. Zerga, 1987. An Analysis of Air Cooling Prior to Reentering a Drift Containing Emplaced High-Level Nuclear Waste, SAND87-7076C, Sandia National Laboratories, Albuquerque, NM, xx pp. (NNA.891013.0221)

Wang, J. S. Y., N. G. W. Cook, H. A. Wollenberg, C. L. Carnahan, I. Javandel, and C. F. Tsang, 1993, "Geohydrologic Data and Models of Rainier Mesa and Their Implications to Yucca Mountain," Proc. Fourth Annual Internat.. Conf., High Level Radioactive Waste Management, Las Vegas, NV, p. 675-681. (NNA. 940203.0038) 
Water, Waste \& Land, Inc., 1986. Analyses of Observed Flow Between Test Wells USWG-1 and USW UZ-1, Nevada Nuclear Waste Storage Investigations, Subtask 1.5, Numerical-Evaluation of Conceptual Models, Mini Report \#6, prepared for the Nuclear Regulatory Commission, 30 November 1986, xx pp. (NN1.880804.0062)

Weeks, E., 1988. "Topographically Affected Airflow in an Open Borehole at Yucca Mountain, Nevada," EOS, Transactions of the American Geophysical Union, vol. 69, p. 1223. (MOL.19940819.0007)

Whitfield, M. S., W. Thordarson, D. P. Hammermesh, and J. B. Warner, 1990, Drilling and Geohydrologic Data for Test Hole USW UZ-1, Yucca Mountain, Nye County, Nevada, U.S. Geological Survey Open-file Report 90354,40 p. (NNA.900622.0450)

Wilson, M. L., J. H. Gauthier, R. W. Barnard, G. E. Barr, H. A. Dockery, E. Dunn, R. R. Eaton, D. C. Guerin, N. Lu, M. J. Martinez, R. Nilson, C. A. Rautman, T. H. Robey, B. Ross, E. E. Ryder, A. R. Schenker, S. A. Shannon, L. H. Skinner, W. G. Halsey, J. Gansemer, L. C. Lewis, A. D. Lamont, I. R. Triay, A. Meijer, and D. E. Morris, 1994, Total-System Performance Assessment for Yucca Mountain-SNL Second Iteration (TSPA1993), SAND93-2675, Sandia National Laboratories, Albuquerque, NM. (NNA.940112.0123)

Winter, E. R. F., and W. O. Barsch, 1971. "The Heat Pipe," in Advances in Heat Transfer, vol. 7, Thomas F. Irvine, Jr., and James P. Hartnett, eds., Academic Press, New York, NY, pp. 219-319. (MOL.19940805.0101)

Younker, J., W. Andrews, G. Fasano, C. Herrington, S. Mattson, R. Murray, L. Ballou, M. Revelli, A. DuCharme, L. Shephard, W. Dudley, D. Hoxie, R. Herbst, E. Patera, B. Judd, J. Docka, and L. Rickertson, 1992, Report of Early Site Suitability Evaluation of the Potential Repository Site at Yucca Mountain, Nevada, SAIC-91/8000, Science Applications International Corporation, Las Vegas, NV. (NNA.910708.0111)

Zhou, W., P. L. Chambre, T. H. Pigford, and W. W. L. Lee, 1990. Heat-Pipe Effect on the Transport of Gaseous Radionuclides Released From a Nuclear Waste Container, LBL-29064, Lawrence Berkeley Laboratory, Berkeley, CA, 11 pp. (MOL.19940805.0095) 


\section{YUCCA MOUNTAIN SITE CHARACTERIZATION PROJECT}

\section{DISTRIBUTION LIST}

1

D.A. Dreyfus (RW-1)

Director

OCRWM

US Department of Energy

1000 Independence Avenue SW

Washington, DC 20585

1 L.H. Barrett (RW-2)

Acting Deputy Director

OCRWM

US Department of Energy

1000 Independence Avenue SW

Washington, DC 20585

1 J.D. Saltzman (RW-4)

Office of Strategic Planning and

International Programs

OCRWM

US Department of Energy

1000 Independence Avenue SW

Washington, DC 20585

1 J.D. Saltzman (RW-5)

Office of External Relations

OCRWM

US Department of Energy

1000 Independence Avenue SW

Washington, DC 20585

1 Samuel Rousso (RW-10)

Office of Program and Resource Mgt.

OCRWM

US Department of Energy

1000 Independence Avenue SW

Washington, DC 20585

1

J. C. Bresee (RW-10)

OCRWM

US Department of Energy

1000 Independence Avenue SW

Washington, DC 20585
1 R.M. Nelson (RW-20)

Office of Geologic Disposal

OCRWM

US Department of Energy

1000 Independence Avenue, SW

Washington, DC 20585

1 S. J. Brocoum (RW-22)

Analysis and Verification Division OCRWM

US Department of Energy

1000 Independence Avenue SW

Washington, DC 2585

1 D. Shelor (RW-30)

Office of Systems and Compliance

OCRWM

US Department of Energy

1000 Independence Avenue, SW

Washington, DC 20585

1 J. Roberts (R'W-33)

Director, Regulatory Compliance

Division

OCRWM

US Department of Energy

1000 Independence Avenue, SW

Washington, DC 20585

1

G. J. Parker (RW-332)

Reg. Policy/Requirements Branch

OCRWM

US Department of Energy

1000 Independence Àvenue, SW

Washington, DC 20585

1

R. A. Milner (RW-40)

Office of Storage and

Transporation

OCRWM

US Department of Energy

1000 Independence Avenue, SW

Washington, DC 20585 
Office of Contract Business

Management

OCRWM

US Department of Energy

1000 Independence Avenue, SW

Washington, DC 20585

1

T. Wood (RW-52)

Director, M\&O Management Division OCRWM

US Department of Energy

1000 Independence Avenue, SW

Washington, DC 20585

4 Victoria F. Reich, Librarian

Nuclear Waste Technical Review Board 1100 Wilson Blvd, Suite 910

Arlington, VA 22209

5 Wesley E. Barnes, Project Manager

Yucca Mountain Site

Characterization Office

US Department of Energy

P.O. Box 98608-MS 523

Las Vegas, NV 89193-8608

1 C. L. West, Director

Office of External Affairs

DOE Nevada Field Office

US Department of Energy

P.O. Box 98518

Las Vegas, NV 89193-8518

8 Technical Information Officer

DOE Nevada Field Office

US Department of Energy

P.O. Box 98518

Las Vegas, NV 89193-8518

1 P. K. Fitzsimmons, Technical Advisor

Office of Assistant Manager for

Environmental Safety and

Health

DOE Nevada Field Office

US Department of Energy

P.O. Box 98518

Las Vegas, NV 89193-8518
1

D. R. Elle, Director

Environmental Protection and Division

DOE Nevada Field Office

US Department of Energy

P.O. Box 98518

Las Vegas, NV 89193-8518

1 Repository Licensing \& Quality

Assurance

Project Directorate

Division of Waste Management

US NRC

Washington, DC 20555

1

Senior Project Manager for Yucca

Mountain

Repository Project Branch

Division of Waste Management

US NRC

Washington, DC 20555

1

NRC Document Control Desk

Division of Waste Management

US NRC

Washington, DC 20555

1

Philip S. Justus

NRC Site Representive

301 E Stewart Avenue, Room 203

Las Vegas, NV 89101

1

E. P. Binnall

Field Systems Group Leader

Building 50B/4235

Lawrence Berkeley Laboratory

Berkeley, CA 94720

1

Center for Nuclear Waste

Regulatory Analyses

6220 Culebra Road

Drawer 28510

San Antonio, TX 78284

3 W. L. Clarke

Technical Project Officer - YMP

Attn: YMP/LRC

Lawrence Livermore National

Laboratory

P.O. Box 5514

Livermore, CA 94551 
J. A. Blink

Deputy Project Leader

Lawrence Livermore National

Laboratory

101 Convention Center Drive

Suite 820, MS 527

Las Vegas, NV 89109

4

J. A. Canepa

Technical Project Officer - YMP

N-5, Mail Stop J521

Los Alamos National Laboratory

P.O. Box 1663

Los Alamos, NM 87545

1 H. N. Kalia

Exploratory Shaft Test Manager

Los Alamos National Laboratory

Mail Stop 527

101 Convention Center Dr., $\$ 820$

Las Vegas, NV 89101

1

N. Z. Elkins

Deputy Technical Project Officer

Los Alamos National Laboratory Mail Stop 527

101 Convention Center Dr., \#820

Las Vegas, NV 89101

\section{S. Costin, Acting}

Technical Project Officer - YMP

Sandia National Laboratories

Organization 6302, M/S 1333

P.O. Box 5800

Albuquerque, NM 87185

1 J. F. Devine

Asst Director of Engineering Geology

US Geological Survey

106 National Center

12201 Sunrise Valley Drive

Reston, VA 22092

1
L. R. Hayes

Technical Project Officer

Yucca Mountain Project Branch

MS 425

US Geological Survey

P.O. Box 25046

Denver, CO 80225
1

\author{
V. R. Schneider \\ Asst. Chief Hydrologist-MS 414 \\ Office of Progiam Coordination \\ and Technical Support \\ US Geological Survey \\ 12201 Sunrise Valley Drive \\ Reston, VA 22092
}

1

J. S. Stuckless

Geologic Division Coordinator

MS 913

Yucca Mountain Project

US Geological Survey

P.O. Box 25046

Denver, CO 80225

1 D. H. Appel, Chief

Hydrologic Investigations Program MS 421

US Geological Survey

P.O. Box 25046

Denver, CO 80225

1 E. J. Helley

Branch of Western Regional Geology MS 427

US Geological Survey

345 Middlefield Road

- Menlo Park, CA 94025

1 R. W. Craig, Chief

Nevada Operations Office

US Geological Survey

101 Convention Center Drive

Suite 860, MS 509

Làs Vegas, NV 89109

1 D. Zesiger

US Geological Survey

101 Conventional Center Drive

Suite 860, MS 509

Las Vegas, NV 89109

1 G. L. Ducret, Associate Chief

Yucca Mountain Project Division

US Geological Survey

P.O. Box 25046

421 Federal Center

Denver, CO 80225 
US Geological Survey

MS 721

P.O. Box 327

Mercury, NV 89023

1 D. A. Beck

Water Resources Division, USGS

6770 S Paradise Road

Las Vegas, NV 89119

$1 \quad$ P. A. Glancy

US Geological Survey

Federal Building, Room 224

Carson City, NV 89701

1 Sherman S.C. Wu

US Geological Survey

2255 N. Gemini Drive

Flagstaff, AZ 86001

1

J. H. Sass - USGS

Branch of Tectonophysics

2255 N Gemini Drive

Flagstaff, AZ 86001

1 DeWayne Campbell

Technical Project Officer - YMP US Bureau of Reclamation

Code D-3790

P.O. Box 25007

Denver, CO 80225

1 J. M. LaMonaca

Records Specialist

US Geological Survey

421 Federal Center

P.O. Box 25046

Denver, CO 80225

1 W. R. Keefer - USGS

913 Federal Center

P.O. Box 25046

Denver, CO 80225

$1 \quad$ M. D. Voegele

Technical Project Officer - YMP

SAIC

101 Convention Center Drive

Suite 407

Las Vegas, NV 89109
2

L. D. Foust

Nevada Site Manager

TRW Environmental Safety

Systems

101 Convention Center Drive

Suite 540, MS 423

Las Vegas, NV 89109

1

C. E. Ezra

YMP Support Office Manager

EG\&G Energy Measurements Inc MS V-02

P.O. Box 1912

Las Vegas, NV 89125

1 Jan Docka

Roy F. Weston Inc

955 L'Enfant Plaza SW

Washington, DC 20024

1 Technical Information Center

Roy F. Weston Inc

955 L'Enfant Plaza SW

Washington, DC 20024

1 D. Hedges, Vice President, QA

Roy F. Weston Inc

4425 Spring Mountain Road

Suite 300

Las Vegas, NV 89102

1 D. L. Fraser, General Manager

Reynolds Electrical \&

Engineering Co, Inc

MS 555

P.O. Box 98521

Las Vegas, NV 89193-8521

1 B. W. Colston, President and

General Manager

Las Vegas Branch

Raytheon Services Nevada

MS 416

P.O. Box 95487

Las Vegas, NV 89193-5487

1 R. L. Bullock

Technical Project Officer - YMP

Raytheon Services Nevada

Suite P-250, MS 403

101 Convention Center Drive

Las Vegas, NV 89109 
PASS Program

Pacific Northwest Laboratories

P.O. Box 999

Richland, WA 99352

1 A. T. Tamura

Science and Technology Division OSTI

US Department of Energy

P.O. Box 62

Oak Ridge, TN 37831

1 Carlos G. Bell Jr

Professor of Civil Engineering

Civil and Mechanical Engineering Dept.

University of Nevada, Las Vegas

4505 S Maryland Parkway

Las Vegas, NV 89154

1 P. J. Weeden, Acting Director

Nuclear Radiation Assessment Div.

US EPA

Environmental Monitoring

Systems Lab

P.O. Box 93478

Las Vegas, NV 89193-3478

$1 \quad$ ONWI Library

Battelle Columbus Laboratory

Office of Nuclear Waste Isolation

505 King Avenue

Columbus, OH 43201

1 T. Hay, Executive Assistant

Office of the Governor

State of Nevada

Capitol Complex

Carson City, NV 89710

R. R. Loux

Executive Director

Agency for Nuclear Projects

State of Nevada

Evergreen Center, Suite 252

1802 N. Carson Street

Carson City, NV 89710
C.H. Johnson

Technical Program Manager Agency for Nuclear Projects

State of Nevada

Evergreen Center, Suite 252

1802 N. Carson Street

Carson City, NV 89710

1 John Fordham

Water Resources Center

Desert Research Institute

P.O. Box 60220

Reno, NV 89506

1 David Rhode

Desert Research Institute

P.O. Box 60220

Reno, NV 89506

$1 \quad$ Eric Anderson

Mountain West Research-

Southwest Inc

2901 N Central Avenue \#1000

Phoenix, AZ 85012-2730

1 The Honorable Cyril Schank

Chairman

Churchill County Board of

Commissioners

190 W First Street

Fallon, NV 89.406

1

Dennis Bechtel, Coordinator

Nuclear Waste Division

Clark County Department of

Comprehensive Planning

301 E Clark Avenue, Suite 570

Las Vegas, NV 89101

1 Juanita D. Hoffman

Nuclear Waste Repository

Oversight Program

Esmeralda County

P.O. Box 490

Goldfield, NV 89013

$1 \quad$ Eureka County Board of Commissioners

Yucca Mountain Information

Office

P.O. Box 714

Eureka, NV 89316 
1

Brad Mettam

Inyo County Yucca Mountain

Repository Assessment Office

Drawer L

Independence, CA 93526

1 Lander County Board of Commissioners

315 South Humbolt

Battle Mountain, NV 89820

1 Vernon E. Poe

Office of Nuclear Projects

Mineral County

P.O. Box 1026

Hawthorne, NV 89415

1 Les W. Bradshaw

Program Manager

Nye County Repository

P.O. Box 429

Tonopah, NV 89049

$1 \quad$ Florindo Mariani

White Pine County Nuclear

Waste Project Office

457 Fifth Street

Ely, NV 89301

1 Judy Foremaster

City of Caliente Nuclear Waste

Project Office

P.O. Box 158

Caliente, NV 89008

1 Phillip A. Niedzielski-Eichner

Nye County Nuclear Waste

Repository Project Office

P.O. Box 221274

Chantilly, VA 22022-1274

1 Jason Pitts

Lincoln County Nuclear Waste

Project Office

Lincoln County Courthouse

Pioche, NV 89043
1 Economic Development Dept.

City of Las Vegas

400 E. Stewart Avenue

Las Vegas, NV 89101

1 Commmunity Planning and

Development

City of North Las Vegas

P.O. Box 4086

North Las Vegas, NV 89030

1 Community Development and

Planning

City of Boulder City

P.O. Box 61350

Boulder City, NV 89006

1 Commission of the European

Communities

200 Rue de la Loi

B-1049 Brussells

BELGIUM

2

M. J. Dorsey, Librarian

YMP Research and Study Center

Reynolds Electrical \&

Engineering Co Inc

MS 407

P.O. Box 98521

Las Vegas, NV 89193-8521

1 Amy Anderson

Argonne National Laboratory

Building 362

9700 S Cass Avenue

Argonne, IL 60439

1 Steve Bradhurst

P.O. Box 1510

Reno, NV 89505

1 Michael L. Baughman

35 Clark Road

Fiskdale, MA 01518

$1 \quad$ Glenn Van Roekel

Director of Community

Development

City of Caliente

P.O. Box 158

Caliente, NV 89008 
1

Ray Williams, Jr

P.O. Box 10

Austin, NV 89310

$1 \quad$ Nye County District Attorney

P.O. Box 593

Tonopah, NV 89049

$1 \quad$ William Offutt

Nye County Manager

Tonopah, NV 89049

1 Charles Thistlethwaite, AICP

Associate Planner

Inyo County Planning Department

Drawer L

Independence, CA 93526

1

R. F. Pritchett

Technical Project Officer - YMP

Reynolds Electrical \&

Engineering Company Inc

MS 408

P.O. Box 98521

Las Vegas, NV 89193-8521

1 Dr. Moses Karakouzian

1751 E Reno \#125

Las Vegas, NV 89119

10 Tim Brady

USGS

P.O. Box 25046

MS421

Denver Federal Center

Denver, CO 80225

$10 \quad$ Allan Flint

USGS

P.O. Box 327

MS721

Mercury, NV 89023

$1 \quad$ R. W. Nelson

M\&O/INTERA

101 Convention Center Drive

Las Vegas, NV 89109
1

Eric Smistad

DOE-YMSCO

101 Convention Center Drive

Las Vegas, NV 89109

$1 \quad$ Russ Patterson

DOE-YMSCO

101 Convention Center Drive

Las Vegas, NV 89109

1

G. Bodvarsson

LBL

Nuclear Waste Department

1 Cyclotron Road

Berkeley, CA 94720

$1 \quad$ Karsten Pruess

LBL

Nuclear Waste Department

1 Cyclotron Road

Berkeley, CA 94720

MS

21330

C. B. Michaels, 6352 100/12541/SAND922186/QA

1330 WMT Library, 6352

1324 P. B. Davies, 6115

0827 P. J. Hommert, 1502

1375 D. A. Dahlgren, 4400

1326 G. E. Barr, 6312

1326 E. Dunn, 6312

0728 R. L. Hunter, 6602

0899 Technical Library, 13414

0619 Print Media, 12615.

0100 Document Processing for DOE/OSTI, 7613-2

9018 Central Technical Files, 8523-2 